\title{
Dynamic Heterogeneous Hydrogels with Cellulose Nanocrystals
}

\author{
Dissertation \\ for the award of the degree \\ "Doctor rerum naturalium" (Dr.rer.nat.) \\ of the Georg-August-Universität Göttingen
}

within the doctoral program "Material Science Wood"

of the Georg-August University School of Science (GAUSS)

\author{
submitted by \\ Heqin Huang \\ from P.R. China
}

Göttingen, 2019 


\section{Thesis Committee}

Prof. Dr. Kai Zhang, Holztechnologie und Holzchemie, Georg-August-Universität Göttingen

Prof. Dr. Philipp Vana, Makromolekulare Chemie, Georg-August-Universität Göttingen

Prof. Dr. Carsten Mai, Abteilung Holzbiologie und Holzprodukte, Georg-August-Universität Göttingen

Prof. Dr. Cynthia A. Volkert, Institut für Materialphysik, Georg-August-Universität Göttingen

\section{Members of the Examination Board}

\section{Reviewer:}

Prof. Dr. Kai Zhang, Holztechnologie und Holzchemie, Georg-August-Universität Göttingen

\section{Second Reviewer:}

Prof. Dr. Philipp Vana, Makromolekulare Chemie, Georg-August-Universität Göttingen

\section{Further members of the Examination Board:}

Prof. Dr. Carsten Mai, Abteilung Holzbiologie und Holzprodukte, Georg-August-Universität Göttingen

Prof. Dr. Cynthia A. Volkert, Institut für Materialphysik, Georg-August-Universität Göttingen

Dr. Florian Rehfeldt, Drittes Physikalisches Institut - Biophysik, Georg-August-Universität Göttingen

Dr. Yong Wang, Abteilung Fluidphysik, Strukturbildung und Biokomplexität, Max-PlanckInstitut für Dynamik und Selbstorganisation

Date of the oral examination: March 14, 2019 


\section{Acknowledgement}

Firstly, I would like to thank my supervisor Prof. Dr. Kai Zhang for his invaluable guidance and support in my $\mathrm{PhD}$ research. In addition to the guidance in research, Prof. Dr. Zhang also gave me a lot of help in life, especially during my very early days in Göttingen. I would say that I am very lucky to have the opportunity to pursue my $\mathrm{PhD}$ degree with this smart and enthusiastic scientist, and these three and half years $\mathrm{PhD}$ period will be an unforgettable and priceless journey in my life.

Also, I would like to thank three other professors in my supervising committee: Prof. Dr. Philipp Vana, Prof. Dr. Carsten Mai and Prof. Dr. Cynthia A. Volkert. I deeply appreciate their generous help in equipment supporting and inspirational comments.

Moreover, I wholeheartedly thank all my collaborators for their stimulating inspiration, valuable discussion and fruitful collaboration. I also greatly thank all my group members and my friends around the world for their sincere and precious friendship. I would like to thank my parents and my beloved wife - Xiaojie Wang for their unconditional love, support, care and encouragement.

Special thanks go to the financial support from "Materialforschung Holz" at the University of Göttingen. 


\begin{abstract}
Most biological tissues are made out of hydrogels, which are three dimensionally (3D) crosslinked polymer chains in an aqueous microenvironment. Comparing with highly dynamic and heterogeneous natural hydrogels, such as muscle or cartilage, traditional synthetic hydrogels usually suffer from poor mechanical properties. Meanwhile, even the viscoelasticity of hydrogels is widely known, but it is lack of more sophisticated and more practical applications.
\end{abstract}

In this study, cellulose nanocrystals (CNC) and surface-modified CNC introduce heterogeneity into synthetic hydrogels. By mimicking the dynamic heterogeneous structure in the natural hydrogels, a class of hydrogels was prepared with a heterogeneous crosslinking network based on the pre-organized dynamic CNC nano-crosslinkers. With the well-designed dynamic CNCpolymer interaction, the hydrogels not only got stiffened, but exhibited the improved tolerance to local defects. Furthermore, in a dually heterogeneous hydrogel, the necking phenomenon was observed and can be controlled by the active CNC nanocrosslinkers, which narrowed the mechanical difference of diverse hydrogel components and stabilized their shapes and mechanical behaviors as hybrids. In addition to the investigation about hydrogel mechanical properties, the liquid behaviors of dynamic heterogeneous hydrogels were utilized to fabricate polymer materials with programmable shapes and microstructures. We successfully integrated the efficacies of both dynamic and static liquid behaviors to construct structural birefringent materials. Thus, dynamic heterogeneous hydrogels can expand the liquid behavior-assisted materials fabrication into various 3D geometries without the limitation of fluid channels.

Based on this study about dynamic heterogeneous hydrogels, we got deeper understanding of structure-performance relationship in hydrogel materials. In addition, the experiences and working mechanisms demonstrated with $\mathrm{CNC}$ can be further applied in the hydrogels with other nanostructures.

This thesis is a cumulative work including 3 publications. All papers were published in peerreviewed journals. The background, the objective of the study, results and discussion of these 
three publications and the conclusion are presented in Sections 1-4. 


\section{Zusammenfassung}

Die meisten biologischen Gewebe sind aus Hydrogelen aufgebaut, welche dreidimensional quervernetzte Polymerketten in wässrigen Mikroumgebungen sind. Verglichen mit hochdynamischen und heterogenen natürlichen Hydrogelen wie beispielsweise Muskeln oder Knorpel, zeigen traditionelle synthetische Hydrogele schlechtere mechanische Eigenschaften. Mittlerweile ist das viskoelastische Verhalten von Hydrogelen weitgehend bekannt, jedoch fehlen hoch entwickelte und praxisnähere Anwendungen.

In dieser Studie wurde Heterogenität in synthetische Hydrogele durch Cellulose-Nanokristalle (CNC) und oberflächenmodifizierte CNCs eingeführt. Durch das Nachahmen der dynamischen heterogenen Strukturen natürlicher Hydrogele wurde eine Klasse von Hydrogelen mit einem heterogen quervernetzen Netzwerk hergestellt, das auf vororganisierten dynamischen CNC Nanoquervernetzern basiert. Mit wohlüberlegten dynamischen CNC-Polymer-Interaktionen wurden die Hydrogele nicht nur versteift, sondern zeigten auch eine höhere Toleranz gegen lokale Defekte. Außerdem konnte in doppelt heterogenen Hydrogelen ein Einschnürungsphänomen beobachtet werden, das durch aktive CNC Nanoquervernetzer kontrolliert werden kann. Die mechanischen Unterschiede diverser Hydrogelkomponenten können eingegrenzt werden und deren Strukturen sowie mechanisches Verhalten als Hybride lassen sich auch hiermit stabilisieren. Anstatt die mechanischen Eigenschaften von Hydrogelen $\mathrm{zu}$ untersuchen wurde das Flüssigverhalten von dynamischen heterogenen Hydrogelen herangezogen, um Polymermaterialien mit programmierbaren Formen und Mikrostrukturen herzustellen. Darüber hinaus wurde die Leistungsfähigkeit von dynamischem und statischem Verhalten erfolgreich integriert um strukturelle Polymermaterialien zu gestalten. Demnach können dynamische heterogene Hydrogele die Herstellung flüssigkeitsverhaltensgestützter Materialien in unterschiedliche dreidimensionale Formen erleichtern, da sie nicht durch Fluidkanäle limitiert sind.

Basierend auf der Studie über dynamische heterogene Hydrogele konnten wir ein tieferes Verständnis der Struktur-Eigenschaften-Beziehung von Hydrogelmaterialien erlangen. Die 
Erfahrung und der Arbeitsmechanismus, die wir mit CNCs gewonnen haben, können auch auf Hydrogele mit anderen Nanostrukturen angewendet werden.

Diese Dissertation ist eine kumulative Arbeit, die drei Veröffentlichungen beinhaltet. Zwei Artikel wurden in Zeitschriften mit Peer-Review-Verfahren veröffentlicht, einer wurde eingereicht und ist „under review“. Der Hintergrund und das Ziel der Arbeit, Ergebnisse und Diskussion der drei Veröffentlichungen und die Schlussfolgerung sind in den Abschnitten 1-4 gezeigt. 


\section{Table of Contents}

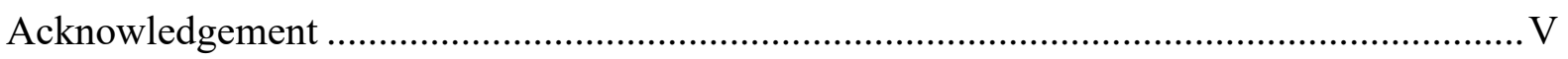

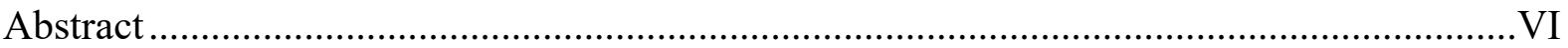

Zusammenfassung................................................................................................ VII

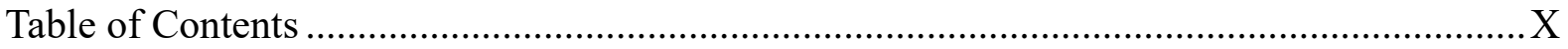

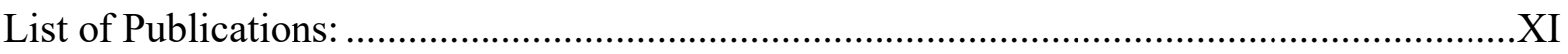

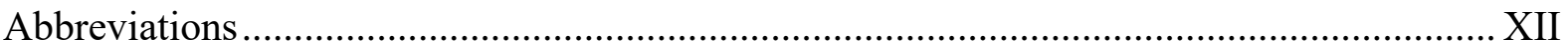

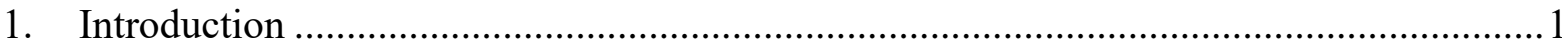

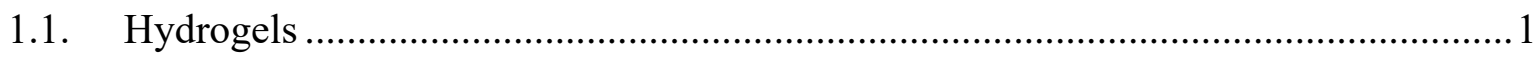

1.2. Mechanical reinforcement of synthetic hydrogels ...................................................2

1.3. Dynamic crosslinks in synthetic hydrogels ............................................................

1.4. Heterogeneous crosslinking networks in synthetic hydrogels ..................................

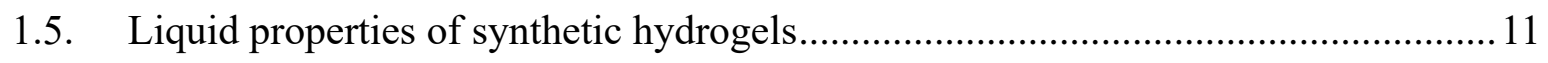

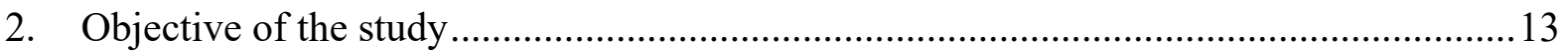

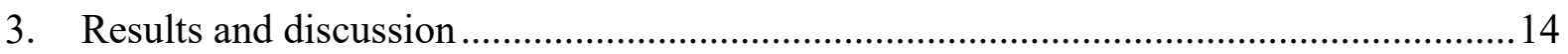

3.1. Robust heterogeneous hydrogels with dynamic nanocrystal-polymer interfaces .....14

3.2. Dually heterogeneous hydrogels with tunable necking phenomenon .........................18

3.3. Liquid behaviors-assisted material fabrication with dynamic hybrid hydrogels ......23

4. General conclusion and perspectives...........................................................................31

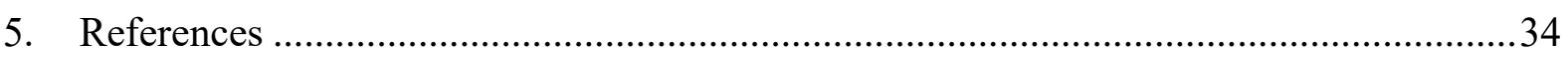

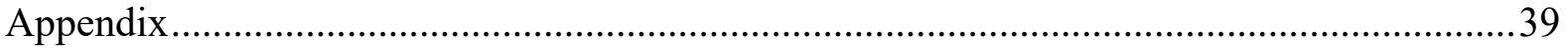

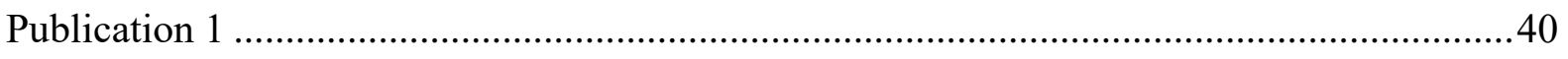

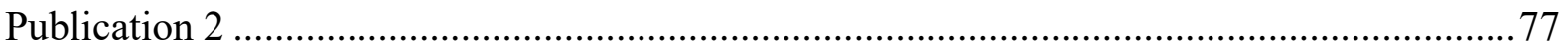

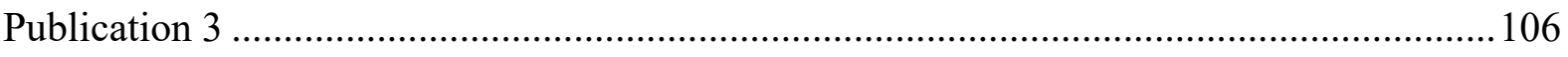

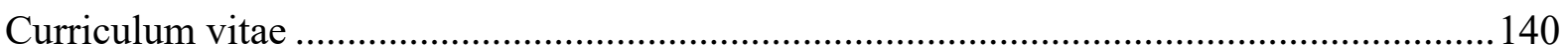




\section{List of Publications:}

Publication 1

Robust heterogeneous hydrogels with dynamic nanocrystal-polymer interface

Heqin Huang, Yonggui Wang, Xiaojie Wang, Florian Rehfeldt, and Kai Zhang*

Macromolecular Rapid Communications 2017, 38, 1600810.

Publication 2

Dually heterogeneous hydrogels via dynamic and supramolecular cross-Links tuning discontinuous spatial ruptures

Heqin Huang, Xiaojie Wang, Florian Rehfeldt, and Kai Zhang*

ACS Sustainable Chemistry \& Engineering 2018, 6 (3), 4294-4301.

Publication 3

Liquid-behaviors-assisted fabrication of multidimensional birefringent materials from dynamic hybrid hydrogels

Heqin Huang, Xiaojie Wang, Jinchao Yu, Ye Chen, Hong Ji, Yumei Zhang, Florian Rehfeldt, Yong Wang, and Kai Zhang*

ACS Nano 2019, DOI: 10.1021/acsnano.9b00551. 


\section{Abbreviations}

$3 \mathrm{D}$

3ITT

AAm (AM)

ADA

AFM

APS

CD

CDI

$\mathrm{CNC}$

$\mathrm{DCH}$

DH

DMA

DMF

LAP

LSM

MCC

NMR

PAAm (PAM)

PAMPS

PBA

PEG

SEM

TEM

TEMED

TEMPO

WAXS

XG
Three dimensional

Three interval thixotropy test

Acrylamide

Adamantane

Atomic force microscopy

Ammonium persulfate

Cyclodextrin

1,1'-carbonyldiimidazole

Cellulose nanocrystals

Dynamic composite hydrogel

Dynamic hydrogel

Dopamine methacrylamide

Dimethylformamide

Lithium phenyl-2,4,6-trimethylbenzoylphosphinate

Laser scanning microscopy

Microcrystalline cellulose

Nuclear magnetic resonance

Poly (acrylamide)

Poly (2-acrylamido, 2-methyl, 1-propanesulfonic acid)

Phenyl boronic acid

Polyethylene glycol

Scanning electron microscopy

Transmission electron microscopy

$\mathrm{N}, \mathrm{N}, \mathrm{N}^{\prime}, \mathrm{N}^{\prime}$-tetramethylethane-1,2-diamine

2,2,6,6-tetramethylpiperidin-1-oxyl

Wide-angle X-ray scattering

Xerogel 


\section{Introduction}

\subsection{Hydrogels}

Hydrogels are 3D hydrophilic crosslinked polymer chains swollen in the water (Figure 1). ${ }^{1}$ The first investigation was back to the late $1950 \mathrm{~s},{ }^{2}$ and now hydrogels have been defined as a remarkable research field. ${ }^{3}$ Highly porous structure of polymer networks in the hydrogels are filled by water, therefore hydrogels naturally have intermediate properties of solid and liquid.

Most of biological tissues, including muscle, mucosa and cartilage of animal, or fruit and leaf of plants are hydrogels. ${ }^{4}$ These natural hydrogels are normally constructed by natural polymers, such as proteins, peptides or polysaccharides, which are crosslinked by highly complexed covalent bonds and non-covalent interactions. In our biosphere, most of life and life behaviors require the presence of water. Therefore, the stable and wet microenvironment provided by hydrogels are extremely important for many biological activities. ${ }^{5}$

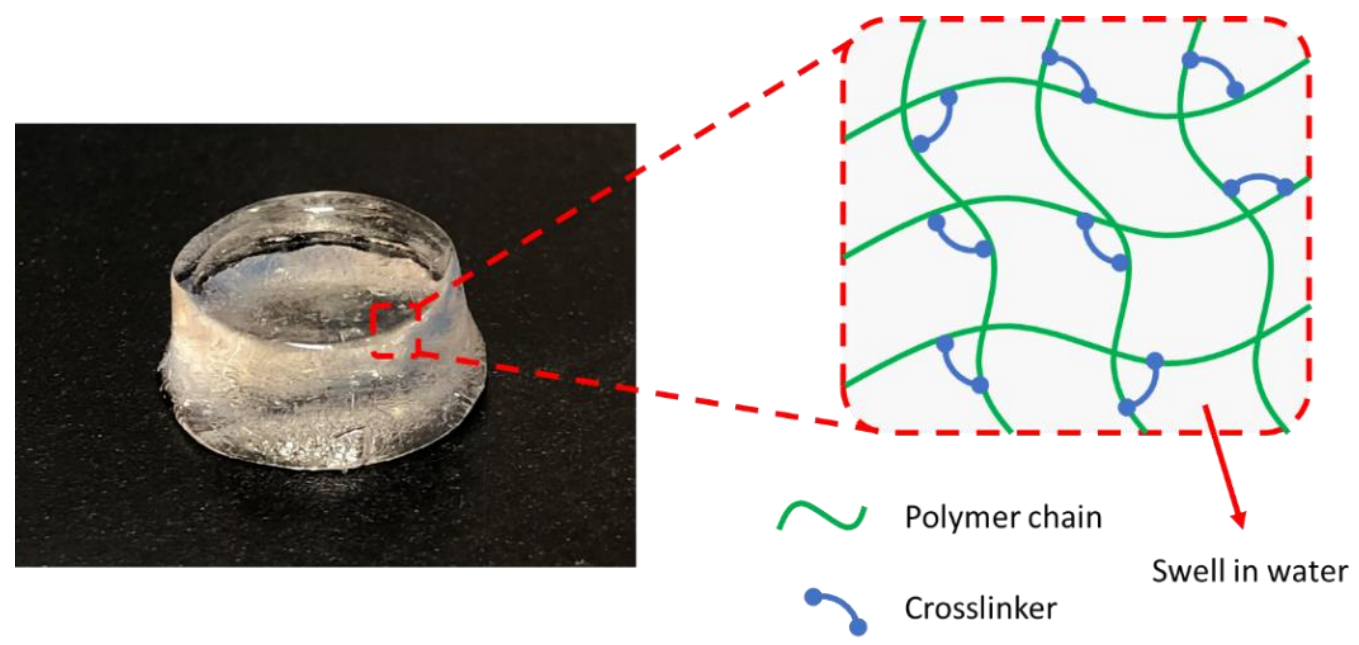

Figure 1. Photo of synthetic hydrogel and schematic illustration of its crosslinking networks.

In the last decades, various artificial hydrogels have been developed to reproduce or even expand the functions of natural hydrogels. Based on the infinite possibilities in polymer chemistry and polymer physics, many kinds of biocompatible and bioactive hydrogels have been developed, which were widely used in the biomedical applications and personal products. ${ }^{6}$ In addition, the well-controlled osmotic pressures and switchable crosslinking networks could 
drive the hydrogel motion under extra stimuli. ${ }^{7}$

Because of high water content and obvious viscoelasticity, hydrogels also exhibit certain liquid behaviors. In the traditional hydrogels with chemical crosslinked networks, the flowing liquid behaviors are largely limited, but the diffusion of water soluble compounds still allows the sustained drug release with hydrogel matrix. ${ }^{8}$ Benefiting from the supramolecular crosslinking, the flowing liquid behaviors now can be created in the physical crosslinked hydrogels. Under the large shearing deformation, dynamic hydrogel crosslinking can partially disassociate and show flowing liquid behaviors. ${ }^{9}$ This shear-thinning phenomenon has been used in the injectable hydrogels ${ }^{10}$ or as viscoelastic ink for 3D printing. ${ }^{11}$

However, comparing with natural hydrogels, traditional synthetic hydrogels usually show poor mechanical properties and are prone to permanent breakage. ${ }^{12}$ Meanwhile, the liquid properties of synthetic hydrogels have not been thoroughly investigated and were lack of more sophisticated applications. ${ }^{13}$

\subsection{Mechanical reinforcement of synthetic hydrogels}

The intrinsic properties of polymer chains, together with the density and kinetics of crosslinking primarily determine the mechanical properties of hydrogels. ${ }^{14}$ However, the molecular defects and highly inhomogeneous microstructure often weaken synthetic hydrogels. ${ }^{15}$

There are two main strategies to improve the mechanical properties of synthetic hydrogels. One is creating defect-free hydrogel networks. ${ }^{16}$ The tetra-PEG hydrogels can be prepared by combining two symmetrical tetrahedron-like macromonomers via click chemistry to form a close-packed hydrogel network (Figure 2). These perfect hydrogel networks aim to homogenize the extra loading to the polymer networks and maximize the extreme strength of hydrogels. With this strategy, hydrogels are highly compressible and show nearly no mechanical hysteresis. With thermal responsive motif, the non-swellable tough hydrogels have been prepared with controllable osmotic pressure in the hydrogels. ${ }^{17}$ Furthermore, with the similar strategy, now the influence of molecular defect to polymer elasticity have been 
quantitatively investigated, which deepened our understanding of the structure-performance relationship in the amorphous materials. ${ }^{15}$ Nevertheless, this ideally homogenous network design allows less flexibility in the fabrication of complexed and multifunctional hydrogels.

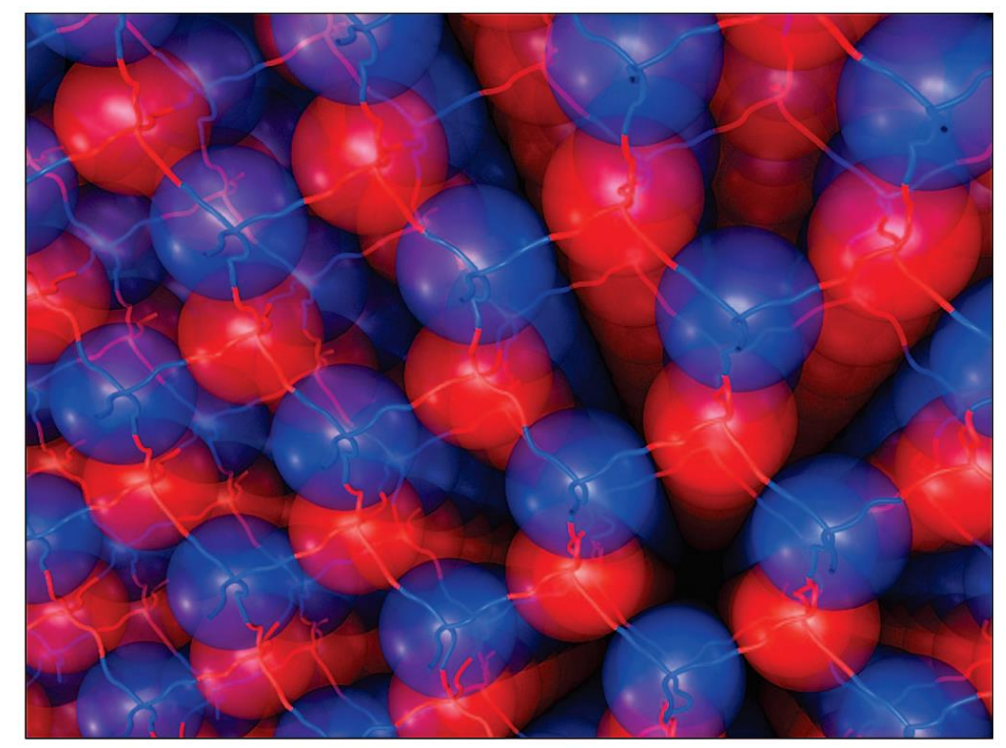

Figure 2. Schematic illustration of a model structure for tetra-PEG gel with close-packed microstructure. Red and blue spheres represent two symmetrical tetrahedron-like macromonomers. (Reproduced from SAKAI et al. ${ }^{16}$ Copyright from American Chemical Society 2008)

Another strategy to toughen the hydrogels is to retard the propagation of local defect with an energy dissipation system. ${ }^{14}$ In this strategy, a sacrificed network or interaction should be preset in the hydrogels, which is able to break or disassociate to absorb the elastic potential energy stored in the deformed polymer networks, and avoid the rapid propagation of local defects. Therefore, the physical integrity of whole hydrogels can be maintained. For example, the wellknown double network hydrogels (Figure 3) ${ }^{18}$ are formed with two interpenetrated crosslinked hydrogel networks. First network is loosely crosslinked, flexible network to maintain the physical integrity and provide elasticity, while the second network is densely crosslinked, which is prone to break and can effectively dissipate energy. Thus, the elastic and tough synthetic hydrogels can be prepared with high water content $(>90 \%)$, but with fracture toughness comparable to some elastomers or thermoplastic polymers. 
(a)

(b)

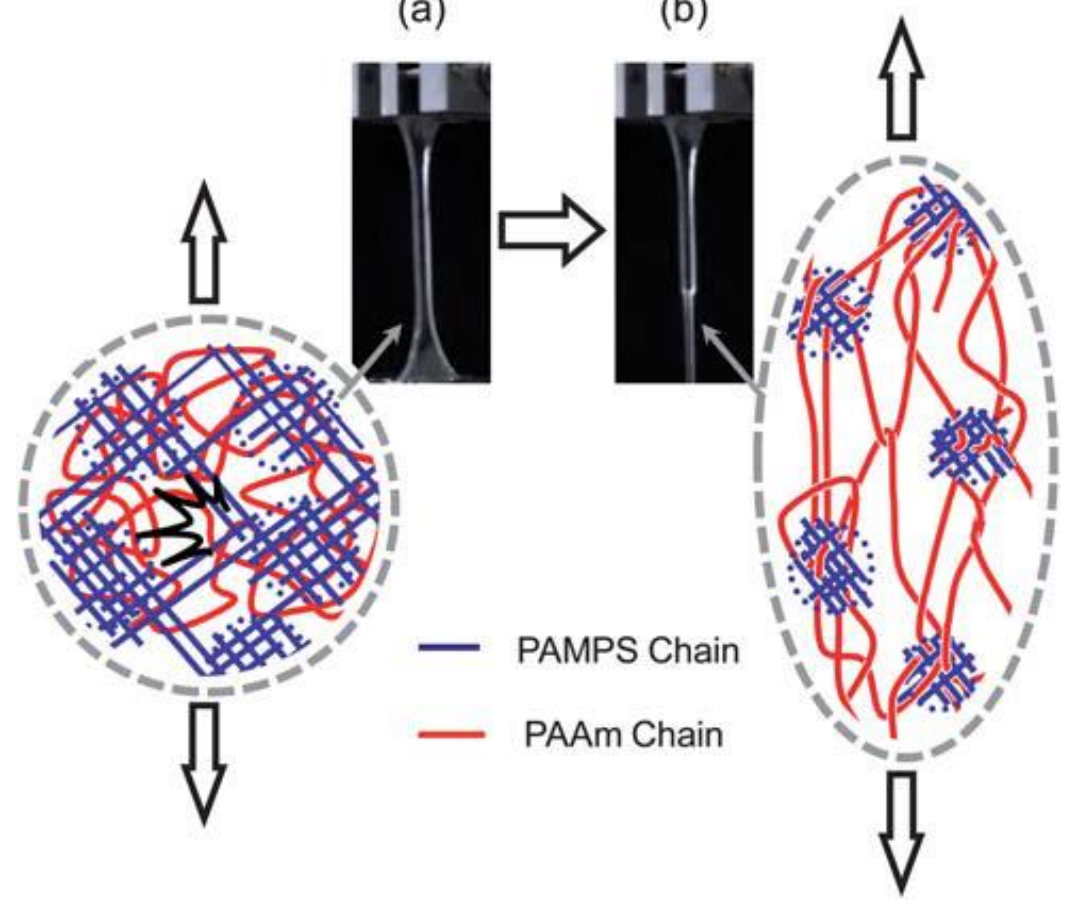

Figure 3. Illustration of the network of the double network hydrogels before (a) and after (b) the breaking of sacrificed secondary poly (2-acrylamido, 2-methyl, 1-propanesulfonic acid) (PAMPS) network. (Reproduced from GONG ${ }^{19}$ Copyright from The Royal Society of Chemistry 2010)

Besides double network hydrogels, several hydrogel systems also follow the similar mechanism. As shown in Figure 4, the slide ring hydrogels, in which the crosslinking points can slide along the polymer chains, and exhibit largely reinforced hydrogel toughness. ${ }^{20,21}$ Polyampholyte hydrogels are crosslinked by polymers with opposite charges, the continuous and gradual disassociation of reversible interaction reinforce the hydrogels. ${ }^{22}$ Nanocrosslinkers in the hydrogels provide multiple connections between two nanocrosslinkers, therefore their stepwise breaking is another kind of energy dissipation system. ${ }^{23}$ In the microscale and macroscale, the fracture and pullout of fibers or fillers can also consume energy in the deformed hydrogels networks. ${ }^{24,25}$ With these energy dissipation systems, heterogeneous microstructure of hydrogels can be maintained, which is critical to enrich the diverse functions to the complexed hydrogels. 
a

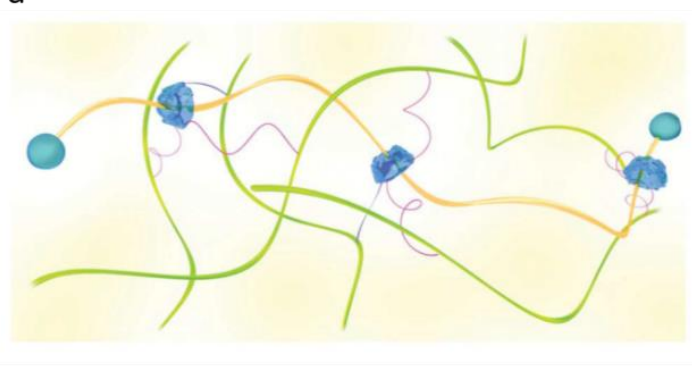

C

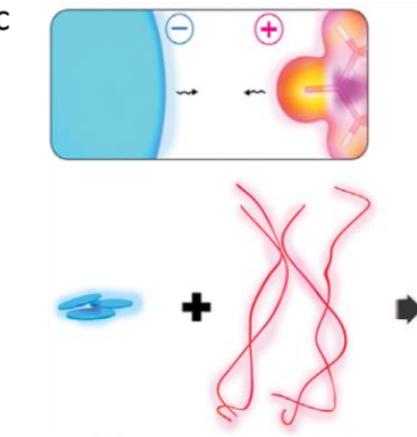

b
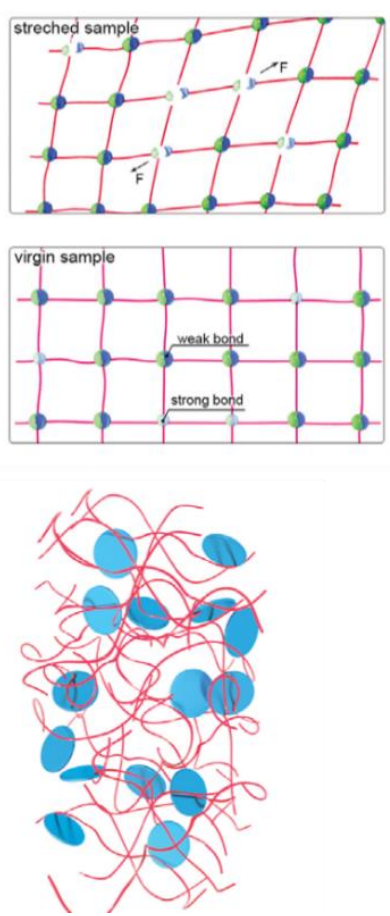

Figure 4. (a) Stretchable hydrogels based on a sliding ring mechanism. (b) Self-healing hydrogels based on polyampholyte design. (c) Composite hydrogels with nanocrosslinking. (Reproduced from ZHANG et al. ${ }^{1}$ Copyright from American Association for the Advancement of Science 2017)

For the tough hydrogels with energy dissipation system, a main problem is their poor antifatigue performance. ${ }^{26}$ These sacrificed interactions are disposable if they are covalent bonds. To overcome this weakness, many reversible interactions have been utilized as the recoverable energy dissipation system. ${ }^{27,28}$ However, the recovery of this energy dissipation system often requires long time ${ }^{29,30}$ or certain treatment ${ }^{31}$, which limits their application under continuous loading.

\subsection{Dynamic crosslinks in synthetic hydrogels}

Covalently crosslinked hydrogels are constructed with permanent polymer networks. In contrast, dynamic crosslinked hydrogels rely on the transient crosslinks of polymer chains which provides a temporary and interchangeable crosslinking network (Figure 5). ${ }^{32}$ Normally, the reversible crosslinking hydrogels often have frequency dependent mechanical properties, 
and they also show shear-thinning phenomenon and self-healing properties. ${ }^{6}$ Therefore, other than the mechanical reinforcement discussed in the last section, the dynamic crosslinking provides more unique functions to the synthetic hydrogels.
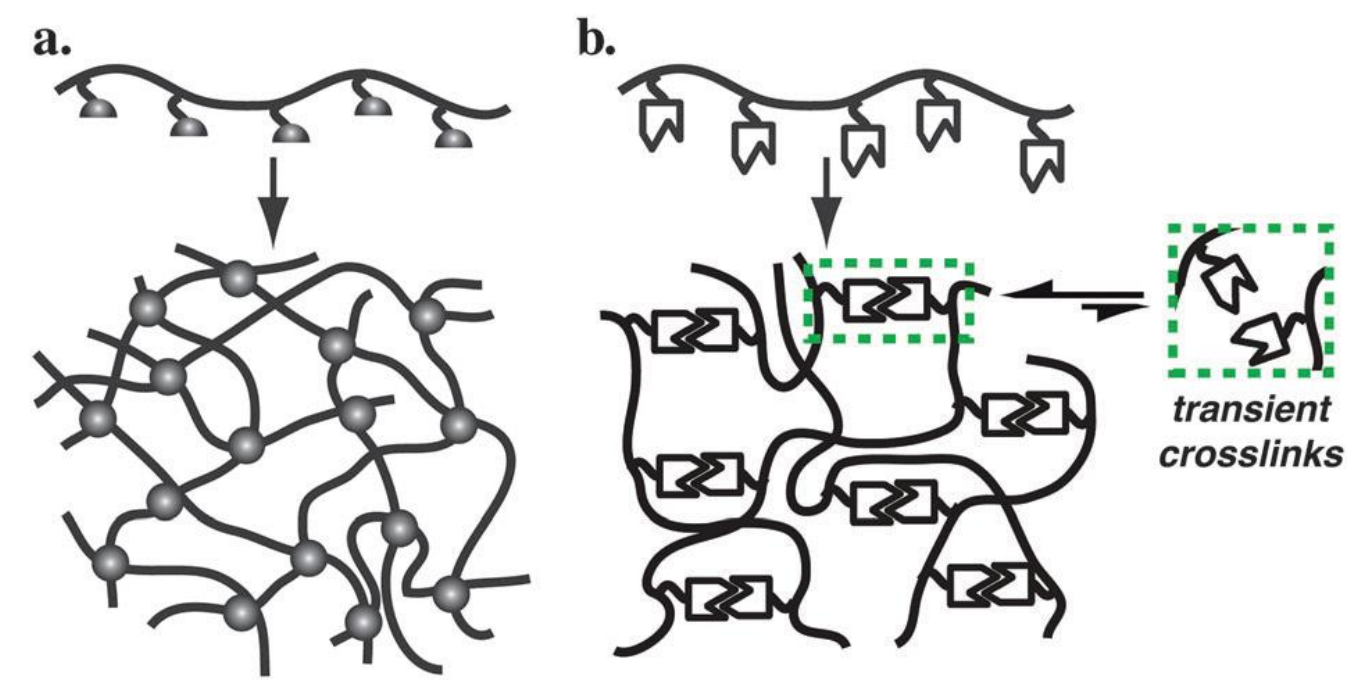

Figure 5. Schematic representation of (a) covalent crosslinking of functional polymer precursors to form static hydrogels and (b) dynamic crosslinking of functional polymer precursors to form transiently crosslinked hydrogels. (Reproduced from APPEL et al. ${ }^{32}$ Copyright from The Royal Society of Chemistry 2012)

Dynamic crosslinks are designed with broad range of binding mechanisms, equilibrium association constants $\left(\mathrm{K}_{\mathrm{eq}}\right)$, and binding dynamics, which is defined by the rate of association $\left(\mathrm{K}_{\mathrm{a}}\right)$ and dissociation $\left(\mathrm{K}_{\mathrm{d}}\right)$ of the moieties. The widely used dynamic interactions include hydrogen bonding, ${ }^{27}$ metal-ligand complexation, ${ }^{33}$ macrocyclic host-guest complexation, ${ }^{34}$ ionic interaction, ${ }^{35-37}$ biomimetic interaction ${ }^{38}$ and diverse dynamic covalent bonds. ${ }^{39-41}$

The dynamic interactions provide the viscoelastic properties of hydrogels. The interchangeable crosslinking networks are favorable to the remold of hydrogels. ${ }^{28}$ Under the high shearing deformation, the disassociated crosslinking network leads to the temporal liquid behaviors of hydrogels, ${ }^{42}$ which allows the injection of hydrogels through the narrow needles. ${ }^{43,44}$ For medical usage, the injectable hydrogels can largely reduce the pain to the patients but still achieve the transformation of implanted materials. Furthermore, hydrogels are widely used as three-dimensional cell culture media. ${ }^{45,46}$ Other than the biocompatibility of hydrogels, 
dynamic crosslinking provides designable and programmable mechanical stimulus to the encapsulated cells, and further guides their biological activities. ${ }^{47}$

The interchangeable dynamic crosslinks also lead to self-healing functions, which can work automatically ${ }^{48}$ or can be controlled by the extra triggers, such as light, ${ }^{49,50} \mathrm{pH}$ value, ${ }^{51,52}$ redox triggers, ${ }^{53}$ temperature ${ }^{54}$ or certain chemicals. ${ }^{41,55}$ The dynamic interactions in the hydrogels not only promote the self-assembly in the molecular level, but also drive the structural selfassembly in the macroscale. ${ }^{56}$

In addition, dynamic crosslinking networks provide the stimulus responsive functions in hydrogels. With stimuli-responsive interactions, the mechanical properties, motions, ${ }^{57}$ and the shape of hydrogels ${ }^{58}$ can be manipulated with external triggers and fulfill the functions such as controllable drug release, ${ }^{23,59}$ or acting as hydrogel soft robots. ${ }^{50,57,60}$

\subsection{Heterogeneous crosslinking networks in synthetic hydrogels}

Biological tissues have highly complex microstructure, which integrate diverse functions and still maintain robust mechanical properties. The real networks in the synthetic hydrogels are typically heterogeneous as well, ${ }^{61}$ where the less cross-linked regions connected by more crosslinked regions. However, unlike the tough natural hydrogels, these synthetic hydrogels exhibit poor tolerance to the local defects. Thus, the heterogeneity solely in the microstructure is not sufficient to construct strong and functional synthetic hydrogels.

Compared with the heterogenous microstructure, the heterogeneous crosslinking networks may take a more important role in the hydrogel toughening. For example, in the hydrogels crosslinked by nanocrosslinkers, ${ }^{62}$ multiple polymer chains between nanocrosslinkers have a large range of length distribution, and their sequentially detachment dissipates energy stored in the hydrogels (Figure 6). ${ }^{63}$ 


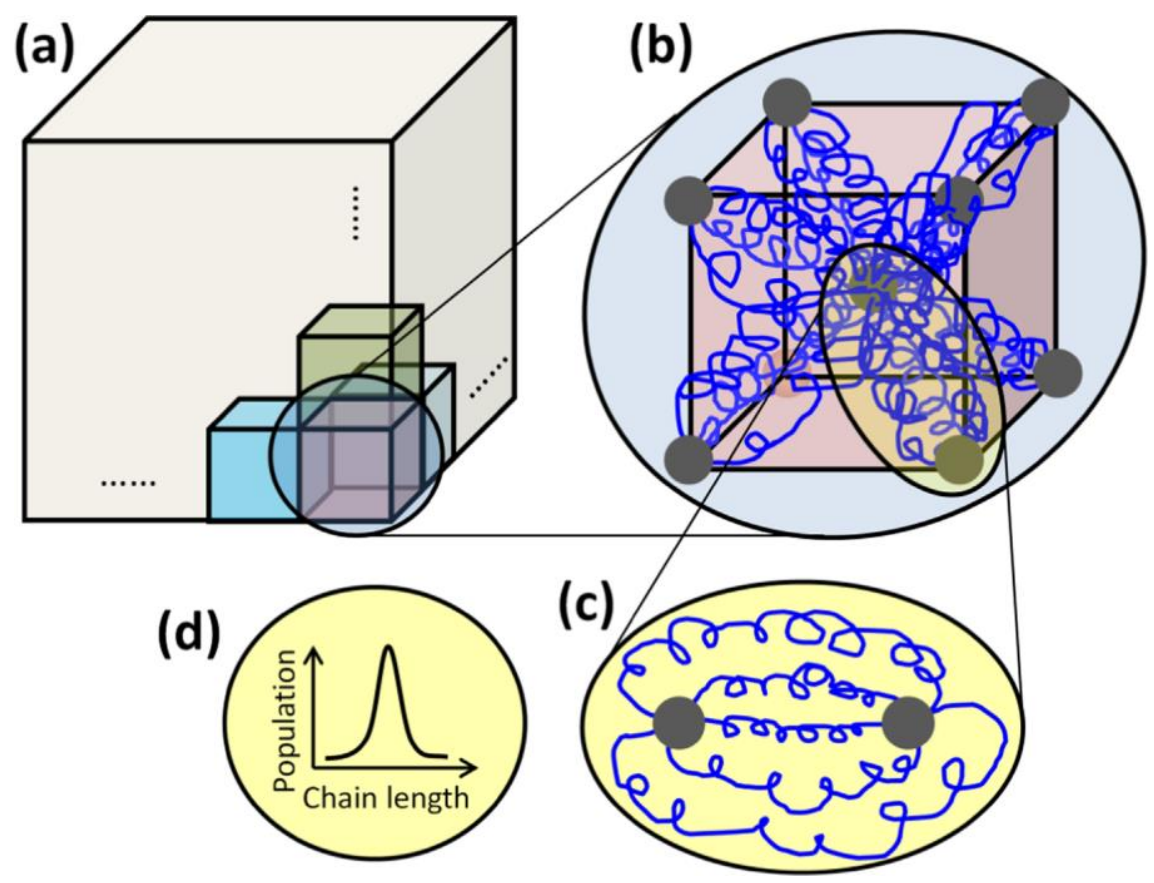

Figure 6. Proposed network model of the nanocomposite hydrogel. (a) The hydrogel consists of layered body-centered cubes. (b) Particle crosslinkers are located on the corners and the center of the cube. (c) Between a particle pair, polymer chains with (d) inhomogeneous lengths are attached on the article surfaces. (Reproduced from WANG et al. ${ }^{63}$ Copyright from Elsevier 2016)

In recent years, various nanoparticle-polymer interactions have been utilized in hydrogels crosslinked by nanocrosslinkers. With covalent crosslinking, the stiff and highly elastic hydrogels were prepared. ${ }^{23,64}$ Meanwhile, supramolecular nanoparticle-polymer interactions were utilized to fabricate dynamic hydrogels with heterogeneous crosslinking networks. ${ }^{65,66}$ The terminal functionalized polymers can be crosslinked by active nanocrosslinkers, in which the hydrogels can be prepared with high structural stability and still maintained the dynamic behaviors from the supramolecular interactions. ${ }^{67}$ As an example, by using dendritic molecular binder with guanidine functional groups, the clay nanosheets were interconnected and formed stiff hydrogels. ${ }^{68}$ In another strategy, the polymer with active side functional groups can be crosslinked by nanocrosslinkers, and the resulting hydrogels are normally very soft and ductile. For example, poly(N-isopropylacrylamide) and clay can form a highly stretchable hydrogels. ${ }^{69}$ The side functional groups of polymer chains provided weak but continuous crosslinking to the nanoparticles, therefore maintained the physical integrity. For a more specific 
supramolecular interaction, three-component host-guest complexes created dynamic interactions between silica nanoparticle and polymers with a high $\mathrm{K}_{\mathrm{eq}}$, but still led to a soft and highly plastic hydrogel, which was favorable to form the tough thin fiber. ${ }^{70}$

Besides the hydrogels directly crosslinked by exogenous nanocrosslinkers, the nanocrosslinking structure can be generated within the prepared hydrogels. The nanoclusters, such as hydrophobic domains ${ }^{71}$ or metal nanoparticles ${ }^{72}$ can be generated in situ in the hydrogels and reinforce dynamic hydrogels.

However, the synergy of nanocrosslinking and small molecular crosslinking is still largely unknown. The influence of large mechanical contrast on the nanoparticle-polymer interface also have not been thoroughly investigated.

Cellulose nanocrystals (CNC) are mechanically strong nanoneedles with native crystalline structures, which can be isolated from wood, cotton, or biosynthesized by bacteria. ${ }^{73} \mathrm{CNC}$ are normally prepared with 100-200 nm in length and $\sim 10 \mathrm{~nm}$ in width (Figure 7). With aligned cellulose chains and highly crystal structures, CNC have extremely high stiffness. ${ }^{74}$ With different isolation method, $\mathrm{CNC}$ can be prepared with various surface functional groups, such as carboxyl groups, aldehyde groups and hydroxyl groups. ${ }^{75}$ These functional groups not only make $\mathrm{CNC}$ easily dispersed in the water, but also allow diverse chemical modifications on $\mathrm{CNC}$, which introduce diverse functionalities to $\mathrm{CNC}$ surface.

In addition to the excellent mechanical properties and great potential in surface modification, $\mathrm{CNC}$ also have unique self-assembly behaviors in the colloidal suspension. ${ }^{76}$ In the water suspension, $\mathrm{CNC}$ tend to form liquid crystal structure with cholesteric phase, which often form tactoid with pitch in tens of micrometer. Furthermore, CNC are highly anisotropic crystal nanomaterials, and can be aligned in the focusing flow to form nematic liquid crystals phase, which is not only favorable for the preparation of extremely strong fibers, ${ }^{77,78}$ but also showed clear birefringence phenomenon. ${ }^{11}$ 


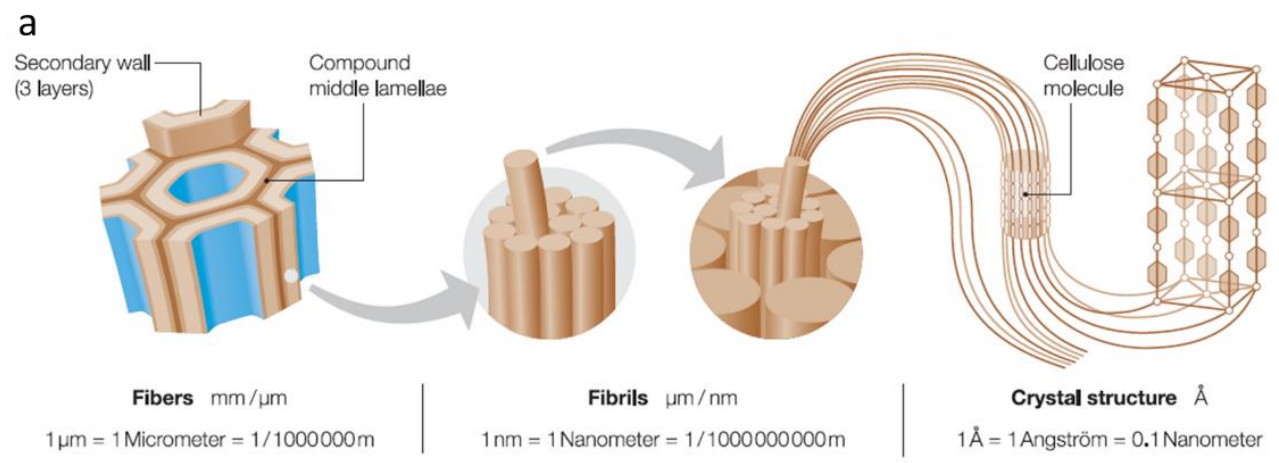

b

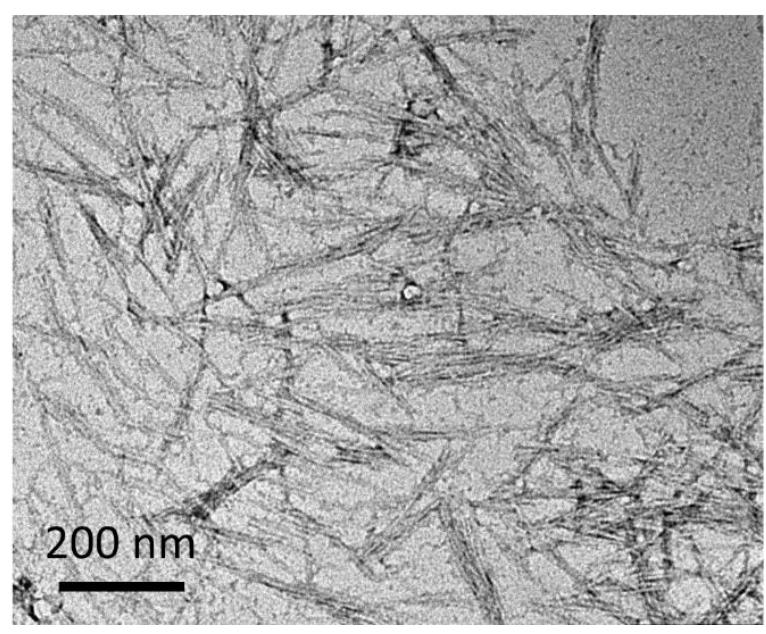

c

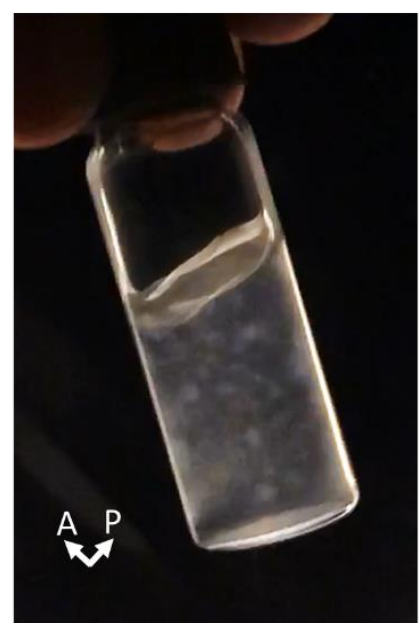

Figure 7. (a) Schematic illustration of the isolation of CNC from wood and plant cell walls. (Reproduced from ZIMMERMANN et al. ${ }^{74}$ Copyright from WILEY-VCH 2004) (b) TEM image of $\mathrm{CNC}$ and (c) polarizing optical image of $\mathrm{CNC}$ water suspension (CNC concentration as $1.6 \mathrm{mg} / \mathrm{ml})$

$\mathrm{CNC}$ have been investigated as nanofillers or nanocrosslinkers in composite hydrogels. Based on the electrostatic interaction between CNCs and methylcellulose, a very weak but thermoresponsive hydrogel has been prepared. ${ }^{79}$ In addition, CNCs were also applied as dynamic nanocrosslinkers to form reinforced composite hydrogel. However, this dynamic bonding was settled in the polymer brush around CNCs, thus, the influence of CNC-polymer interface was not shown directly. ${ }^{80}$ In another case, the $\mathrm{CNC}$ were directly prepared as chemical nanocrosslinkers, and constructed the highly elastic hydrogels with outstanding antifatigue properties. $^{81}$ 
The CNC can be easily modified and applied as diverse nanocrosslinkers, so CNC can be used as ideal model nanoparticles to investigate the heterogenous microstructures in the hydrogels. Functional CNC may not only provide the inhomogeneous crosslinking networks, but also generate a CNC-polymer nanointerface with high mechanical contrast. Thus, the dynamic heterogeneous hydrogels with $\mathrm{CNC}$ would provide an opportunity to illustrate the functions of the dynamic nanointerface in hydrogel systems.

\subsection{Liquid properties of synthetic hydrogels}

With crosslinked polymer networks and high water content, hydrogels naturally have intermediated properties of solid and liquid. However, the liquid property of hydrogels has not been thoroughly considered and utilized in the materials fabrication.

The liquid-solid transition plays an important role in the material processing. ${ }^{82}$ The liquidus precursor is not only favorable for the injection molding, but also allows the manipulation of polymer structure in microscale and macroscale. ${ }^{83}$ On one hand, the anisotropic nanoparticles can be aligned with the running flow, which allows the fabrication of fibers and films with highly anisotropic mechanical properties. ${ }^{13,84}$ On the other hand, in a static liquid system, the surface tension tends to drive a smallest surface area, which is favorable for the preparation of materials with certain shape and curved surface. ${ }^{85,86}$ Furthermore, in the drying process of a static liquid system, the shrinking liquid surface would confine the involatile components in the liquid system and guides their assembly process. ${ }^{87,88}$

However, the application of liquid behaviors is facing several challenges. Firstly, the flowassisted alignment largely relies on the focusing channels, which restricts its application in fabricating complex 3D materials. ${ }^{89}$ Furthermore, the flowing liquid is highly dynamic and transient, while the surface tension-assisted materials fabrication normally requires a static condition. This natural conflict makes it difficult to integrate the full advantages of liquid behaviors in materials fabrication.

So far, a few works have investigated the materials fabrication based on the liquid behaviors of dynamic hydrogels. Relied on the shear-thinning phenomenon, these researches mainly 
focused on the flow-assisted alignment with the dynamic liquid behaviors (Figure 8), ${ }^{11,90}$ while the surface tension-assisted materials manipulation of hydrogels gained less attention.
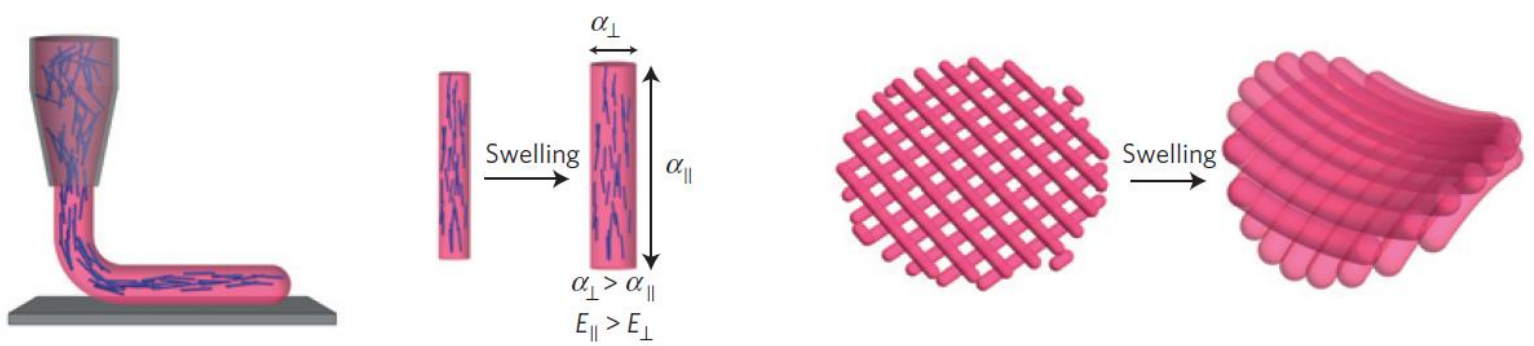

Figure 8. Schematic of the shear-induced alignment of cellulose fibrils during direct ink writing and the 4D printing with the help of swelling process. (Reproduced from GLADMAN et al. ${ }^{90}$ Copyright from Nature Publishing Group 2016)

By combining dynamic hydrogels with $\mathrm{CNC}$, we could exploit the efficacy of shear-thinning phenomenon of dynamic hydrogels and create anisotropic mechanical properties in the resulted materials. Furthermore, because of the high birefringence of $\mathrm{CNC}$, the ordered microstructure would generate controllable interference colors. In addition, the dynamic hydrogels with CNC could sequentially combine the efficacies of flowing liquid and static liquid in the materials fabrication, which can expand the liquid behaviors-assisted materials fabrication to 3D geometry and get rid of the dependency of fluid channels. 


\section{Objective of the study}

Hydrogels are ubiquitous in nature as biological tissues, which are crosslinked polymer networks swollen in water. Therefore, viscoelastic hydrogels often show intermediate properties of solid and liquid.

Normally, hydrogels are taken as solid materials in the swollen state. Natural hydrogels, such as muscles and cartilages, often have highly organized microstructures, robust mechanical properties and can fulfill complex functions. However, traditional synthetic hydrogels are not only suffering from the weak mechanical properties, but also facing the challenge to combine structures with uneven mechanical properties, which is necessary to integrate multiple functions.

Furthermore, over the decades of research, their liquid behaviors of hydrogels, including dynamic liquid properties, and static liquid behaviors, have been largely underestimated. Comparing with covalently crosslinked hydrogels, the dynamic hydrogels reveal more liquid properties, but there is less investigations about their applications and working mechanism in material fabrication.

Therefore, the aim of the present study is to develop synthetic hydrogels with dynamic heterogeneous microstructure. The main objective covers the following points:

1. To prepare diverse functional $\mathrm{CNC}$, and to use them as heterogeneity providers in the synthetic hydrogels. (Publication 1 and 2)

2. To prepare mechanically tough hydrogels with dynamic CNC nanocrosslinkers. (Publication 1)

3. To illustrate the synergism of dynamic crosslinking with small molecular crosslinkers and $\mathrm{CNC}$ nanocrosslinkers, and to integrate hybrid hydrogels with uneven mechanical properties. (Publication 2)

4. To investigate liquid properties of dynamic hydrogels with $\mathrm{CNC}$, and to utilize these liquid properties to construct functional and structural polymer materials. (Publication 3) 


\section{Results and discussion}

\subsection{Robust heterogeneous hydrogels with dynamic nanocrystal-polymer interfaces}

As reported in Publication 1, a kind of stretchable and compressible hydrogels were prepared via host-guest interaction between cellulose nanocrystals (CNC) and hydrogel polymer chains. $\mathrm{CNC}$ as water dispersible nanoparticles, not only have high stiffness and tensile stress, but also have a modifiable surface. As shown in Figure 9, via the four-steps surface modification, the pre-organized dynamic nanocrosslinkers were prepared. The polymerizable functional groups, acrylates or acrylamides were immobilized on the surface of CNC with dynamic host-guest interaction between $\beta$-cyclodextrin/adamantane ( $\beta$-CD/ADA).

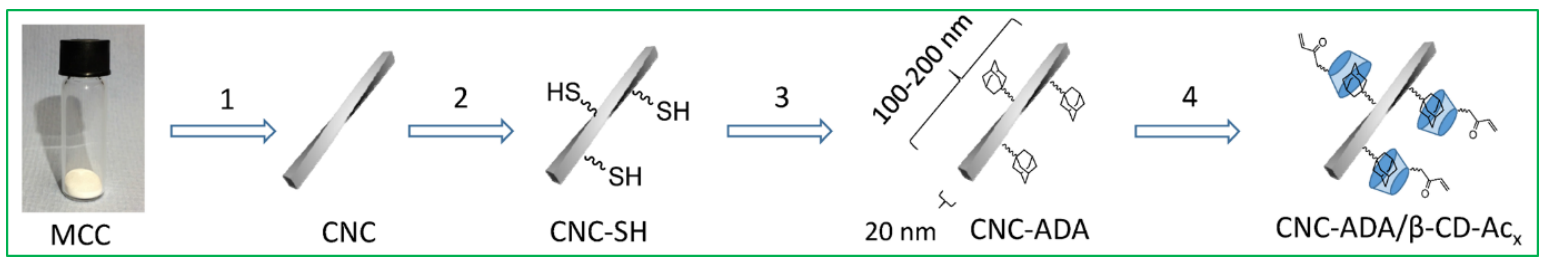

Figure 9. Schematic illustration of the pre-organized dynamic nanocrosslinker.

The dynamic nanocrosslinkers, together with monomer acrylamide (AAm), were polymerized and formed the self-standing hydrogels via an in situ polymerization (Figure 10). In the resulted hydrogels, $\mathrm{CNC}$ were used as the stiff and crystalline nanocrosslinkers, while polyacrylamide (PAAm) was used as the soft and amorphous part. These two components were connected via pre-organized host-guest conjunctions between $\beta-\mathrm{CD}$ and $\mathrm{ADA}$. CNC not only acted as dynamic nanocrosslinkers, but also gathered high density of polymers around them and provided heterogeneity. The pre-organization approach achieved a better bonding efficiency between $\beta$-CD and ADA. In this work, dynamic composite hydrogels (DCH) were investigated both in the as-prepared state and in the fully swollen state.

The reversible $\beta-\mathrm{CD} / \mathrm{ADA}$ conjunctions and their gradual dissociation on the nanocrystalspolymer interface, provided the main energy dissipation system, and consumed the elastic energy stored in the deformed polymer chain. Therefore, the dynamic heterogeneous hydrogels were prepared with high compressibility and stretchability (Figure 11). While the chemical 
crosslinked hydrogels only can withstand very small deformation.

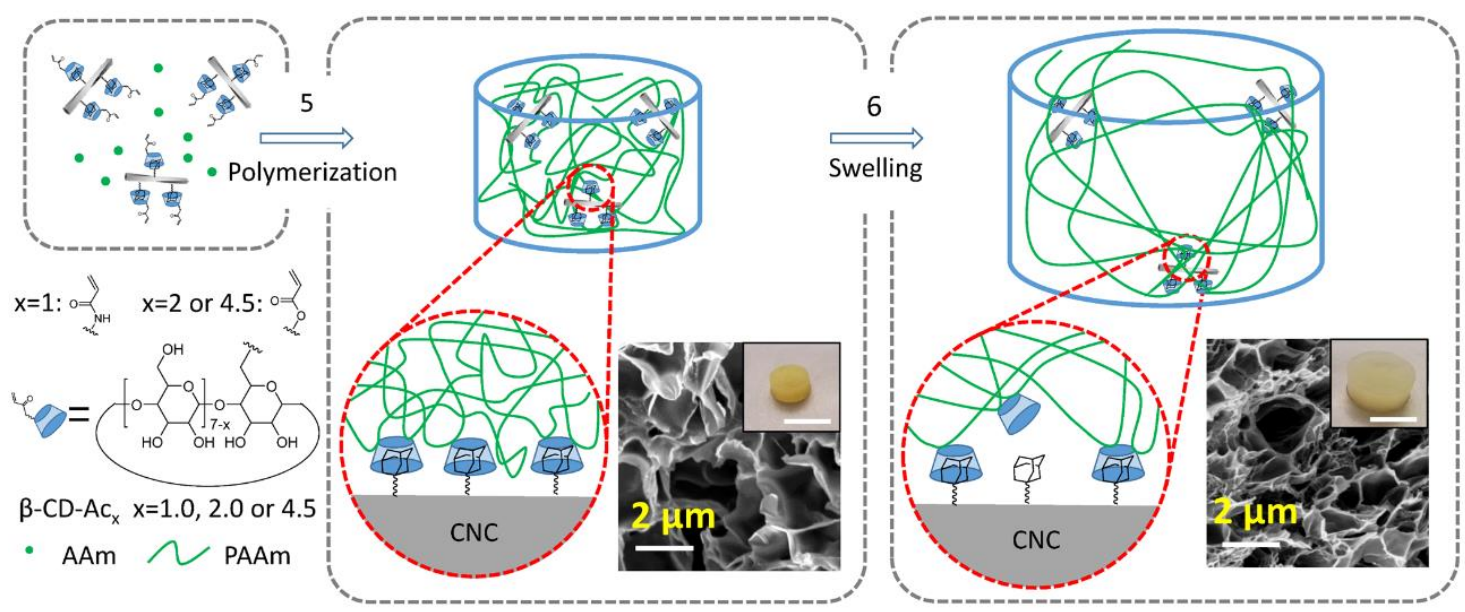

Figure 10. Preparation of dynamic hydrogels with heterogeneous structure (scale bar of hydrogels: $10 \mathrm{~mm}$ ).
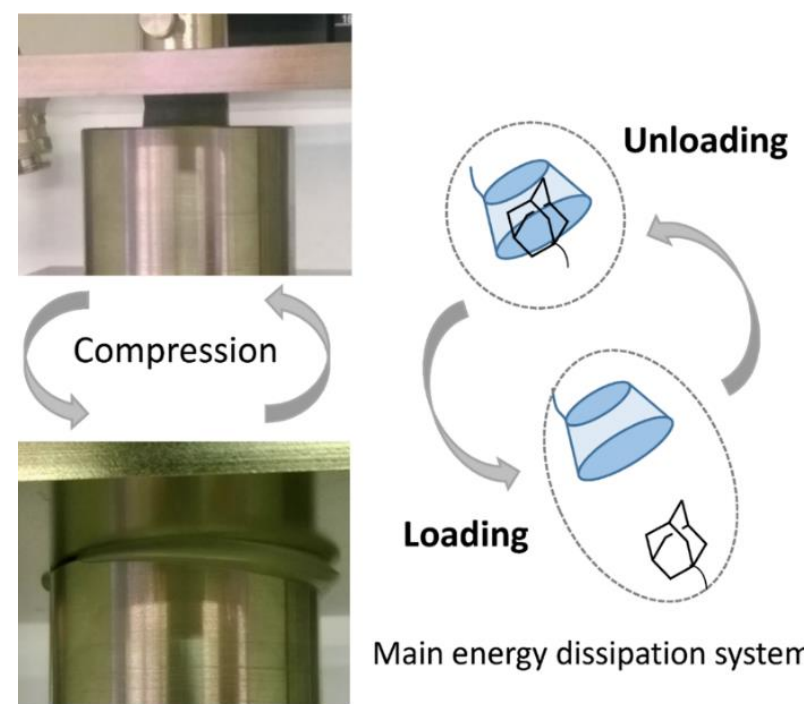

Main energy dissipation system

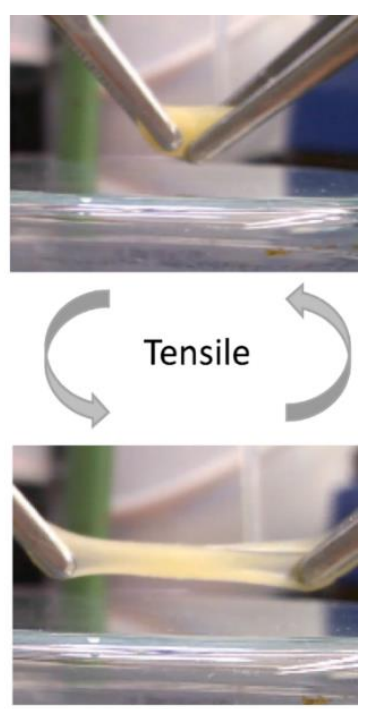

Figure 11. Highly compressible and stretchable hydrogels with dynamic heterogeneous structure.

Benefiting from the reversible nature of $\beta-\mathrm{CD} / \mathrm{ADA}$ conjunctions, this energy dissipation system in the as-prepared hydrogels can be recovered after certain interval times (Figure 12). Since the polymer networks in the hydrogels were in situ polymerized around the CNC, the host-guest interactions would automatically return to their original position. However, in the swollen hydrogels, the toughening mechanism became disposable. The $\beta$-CD/ADA 
conjunctions still worked as energy dissipation system in the first compression or tensile cycle, but the host-guest interaction consumed in the first cycles were not able to rebuild.
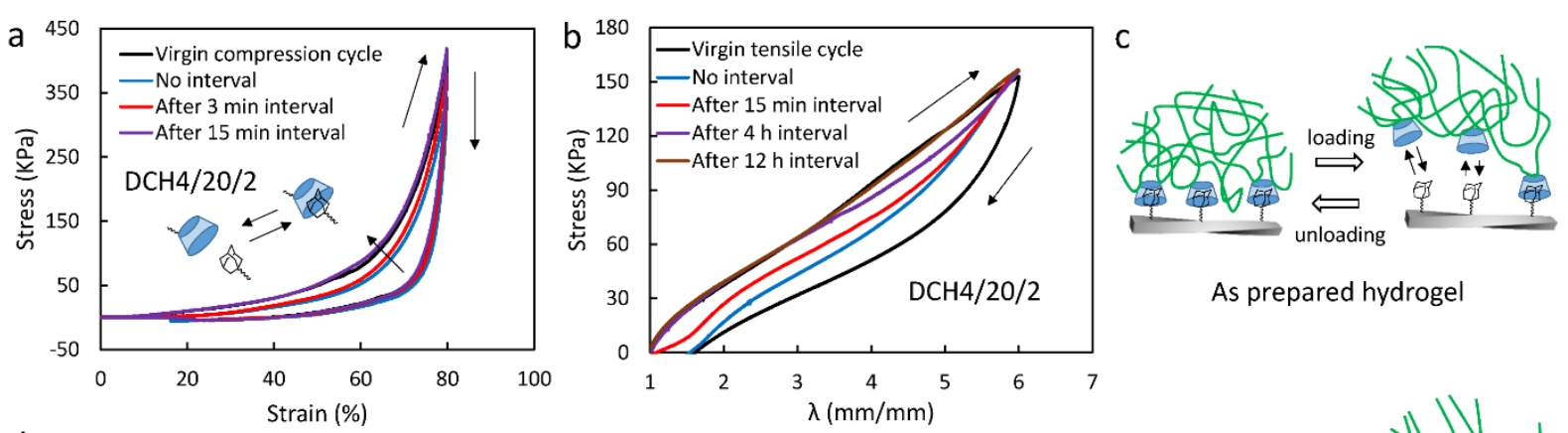

As prepared hydrogel
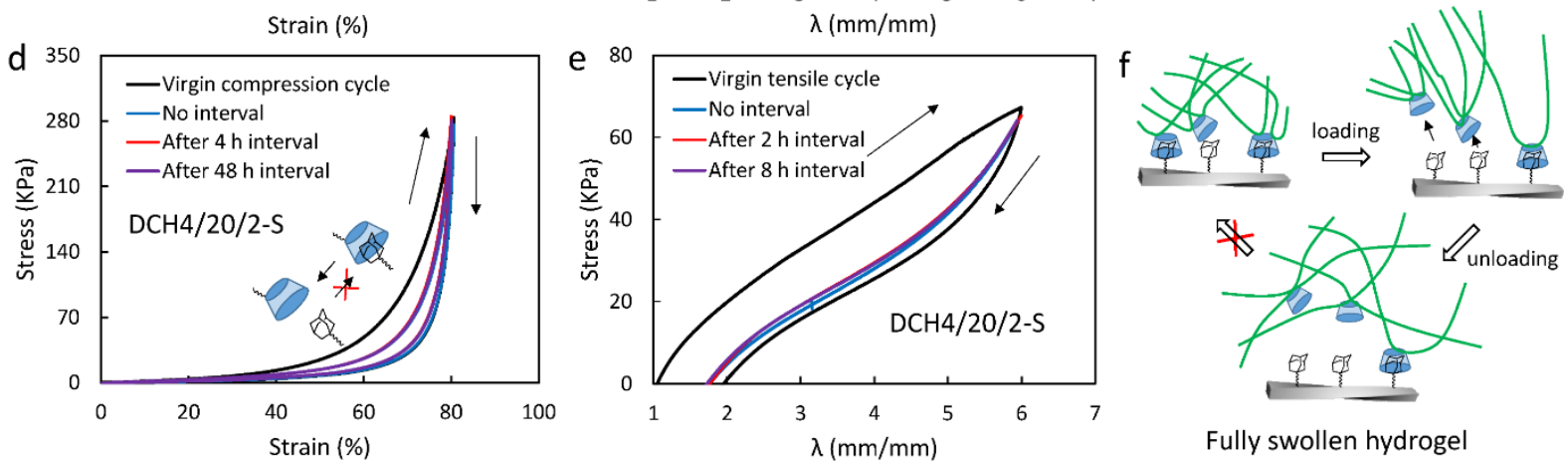

Figure 12. The recovery behaviors of mechanical toughening system in the hydrogels.

During the swelling process, the volume of hydrogels expanded and the distance between CNC nanocrosslinkers increased, so the polymer chains between nanocrosslinkers were stretched. The inhomogeneous swelling subsequently generated the stress concentration on the nanocrosslinker/polymer interface. In our hydrogels, the $\beta$-CD/ADA complexes undertook the stress concentrated on the interface. This residual stress in fully swollen hydrogels would push $\beta-\mathrm{CD} / \mathrm{ADA}$ conjunctions away from equilibrium conditions and intend to dissociate. Therefore, once the host and guest molecules departed from each other, they would not return to their original position.

Besides the improving mechanical properties, the dynamic nano-crosslinkers within the hydrogels also significantly retarded the propagation of the crack (Figure 13). The crack propagation during the stretching of pre-notched hydrogels was paused and retarded until the complete breakup. This could be attributed to the dynamic bonding on the CNC surface. The sequential dissociation of the $\beta-\mathrm{CD} / \mathrm{ADA}$ complexes and the heterogeneous hydrogel structures effectively retarded the crack propagation. 


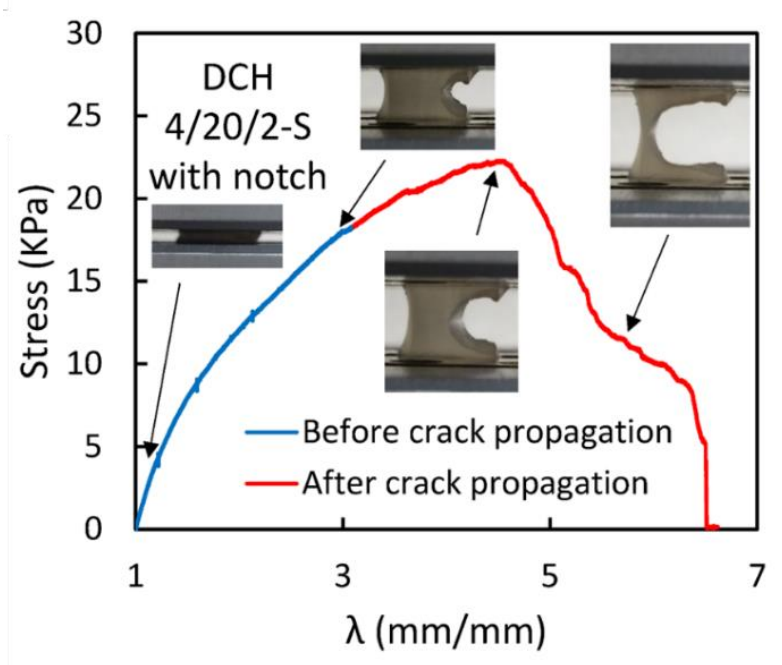

Figure 13. Tensile stress-strain curve of the hydrogel with preset notch.

In the present section, we developed a kind of dynamic composite hydrogel with the heterogeneous structure based on the preorganized dynamic CNC nanocrosslinkers. Acrylated $\beta-\mathrm{CD}$ and $\mathrm{CNC}-\mathrm{ADA}$ formed dynamic nanocrosslinkers via preorganized host-guest interaction. These various $\beta-\mathrm{CD}$ on $\mathrm{CNC}$ surface then polymerized with monomers and constructed hydrogels via purely physical or hybrid chemical/physical interactions. During the hydrogel deformation, this dynamic conjunctions on the CNC-polymer interface exhibited gradual detachment under stress and worked as an effective mechanical toughening system. This toughening mechanism showed a time-dependent recoverability in the as-prepared hydrogels. However, this recoverability was lost when the hydrogels became fully swollen, even they were still toughened by the same energy dissipation system. This phenomenon was due to the highly heterogeneous structure caused by swelling. Along with the volume increase, uneven expansion of nanocrosslinkers and flexible polymers generated residual stress on the CNC-polymer interface, and therefore resisted the recovery of the sacrificed $\beta-\mathrm{CD} / \mathrm{ADA}$ conjunction.

Furthermore, these dynamic nanocrosslinkers within the hydrogels also significantly delayed the propagation of the crack. The crack propagation during the stretching of pre-notched hydrogels was full of struggle, which required much longer further stretching and consumed a large amount of energy. Thus, the heterogeneous microstructure effectively retarded the crack 
propagation of hydrogels and promoted their tolerance to local defect. Based on these, we demonstrated that a dynamic nanocrystal-polymer interface led to tough hydrogels with heterogeneous structures that will be useful for a wide range of purposes.

\subsection{Dually heterogeneous hydrogels with tunable necking phenomenon}

Biological tissues are often highly and multiply heterogeneous in both microstructure and composition in macroscale, but the integrity of multi-heterogeneity in multiple scales in artificial materials is still a big challenge. In the publication 2, dually heterogeneous hydrogels were constructed with two distinct strategies via dynamic bonds and supramolecular crosslinks. The primary heterogeneity resulted from non-uniform distribution of dynamic and/or static crosslinks. Further introduction of the secondary heterogeneity by incorporating anisotropic cellulose nanocrystals (CNC) into the hydrogels provided extra supramolecular crosslinks in the microscale. Benefiting from the synergism of this dually heterogeneous structure, the regulation of deformation and fracture mode of hydrogels was achieved.
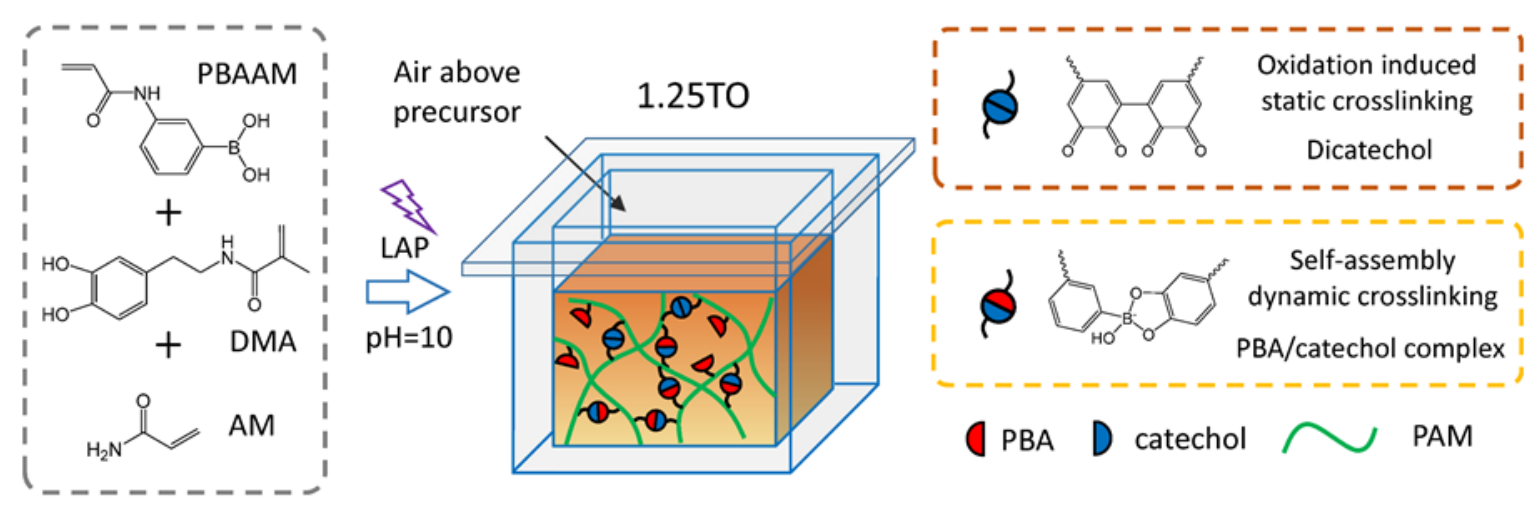

Figure 14. Schematic illustration for the preparation of top-oxidized hydrogels with primary heterogeneity.

In the present work, the dynamic covalent bonds between phenylboronic acid and catechol were applied to form dynamic boronate ester bonds and reversibly crosslinked hydrogels. However, the in situ polymerization in the presence of air would promote the formation of dicatechol, which acted as a static crosslinking for the hydrogels. Therefore, the primary 
heterogeneous hydrogels were prepared with static crosslinked top layer and a dynamic crosslinked matrix (Figure 14).

The static and dynamic crosslinking mechanism reasonably led to different mechanical properties (Figure 15 A, B). The stretched non-oxidized 1.25NO would not break until the end of the tensile test machine ( $>2000 \%$ ), and the fully-oxidized $1.25 \mathrm{FO}$ was elastic and brittle and broke at about $300 \%$ elongation. Therefore, in the top-oxidized hydrogel $1.25 \mathrm{TO}$, the mechanical difference led to a necking phenomenon during the uniaxial stretching: during the stretching with the elongation ratio as early as of 100-150\%, multiple necking points appeared (Figure $15 \mathrm{C}$ ). The non-necking zones and necking zones were both extend during the further stretching, but the necking zones apparently undertook more deformation. The propagated primary necking points (red region) and secondary necking points (blue arrows) were highlighted in the amplified photo of $200 \%$ stretched $1.25 \mathrm{TO}$. The necking maintained and extended during further stretching at higher elongation ratios of $600 \%$ and $1000 \%$. Thus, the non-uniform distribution of the static dicatechol bonds and dynamic PBA/catechol crosslinks within the diverse regions in hydrogels in fact led to the heterogeneity in macrostructures.

Furthermore, another kind of heterogeneity in microscale can be constructed by establishing supramolecular interactions between nanoparticles and polymer chains. As shown in the Figure 16, we used anisotropic nanocellulose with two distinct surface functionalities: CNC (TEMPO-oxidized) with only carboxyl groups $(1.52 \pm 0.03 \mathrm{mmol} / \mathrm{g})$ on the surface and CNCPBA with surface-grafted phenylboronic acid groups $(1.07 \pm 0.05 \mathrm{mmol} / \mathrm{g})$ in addition to the rest of carboxyl groups $(\sim 0.4 \mathrm{mmol} / \mathrm{g})$. The neutral CNC only provided weak and nonspecific crosslinks to the surrounding hydrogel networks and the active CNC-PBA would provide stronger and more specific dynamic crosslinks with the catechol groups in the hydrogels. 

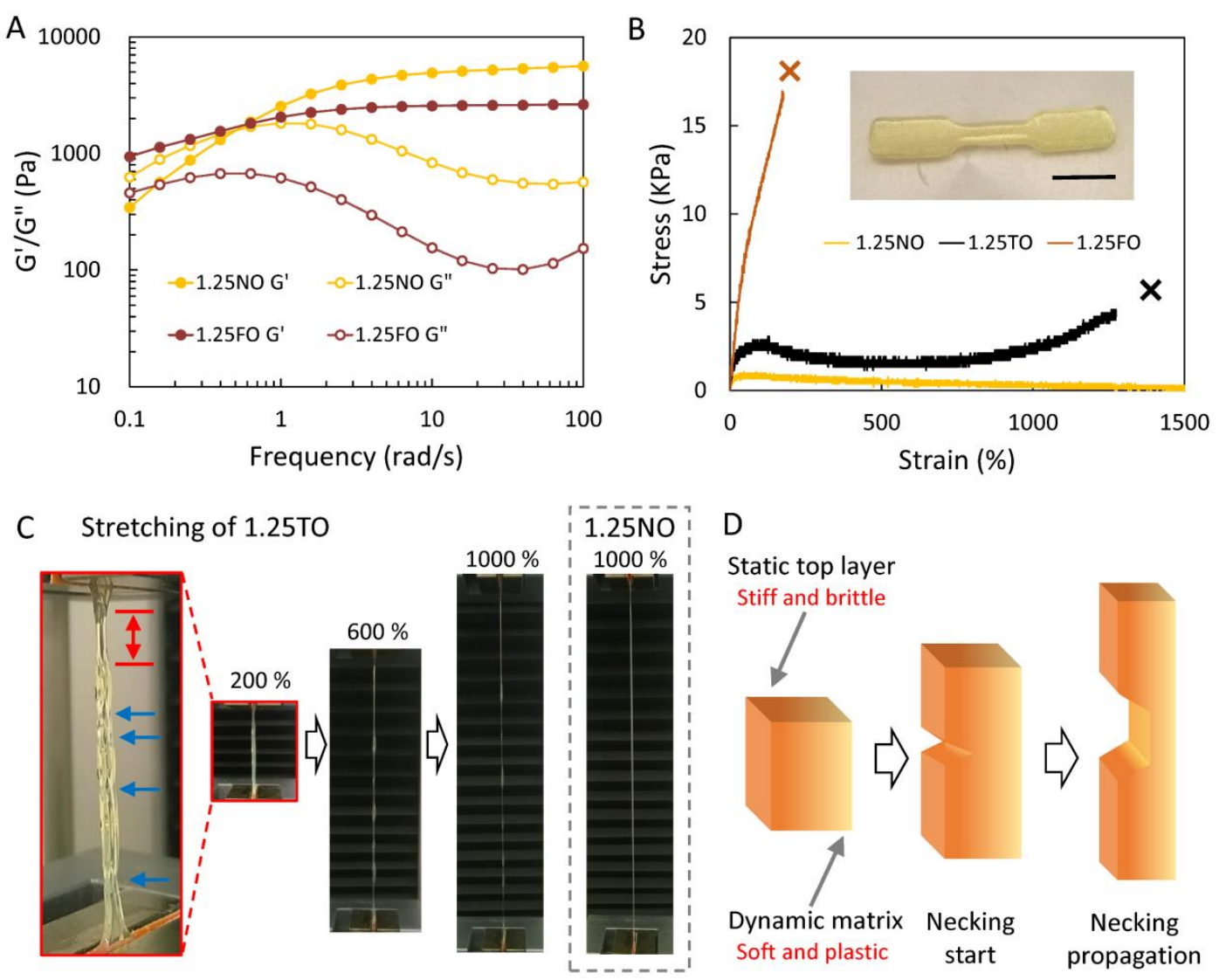

Figure 15. The heterogeneous mechanical features of top-oxidized hydrogels and their necking deformation during stretch.

To further understand different effects of CNC and CNC-PBA in the hydrogels, CNC and CNCPBA were separately investigated in the non-oxidized hydrogels and fully oxidized hydrogels, which could exclude the interference of the primary heterogeneity (Figure 17). In the hydrogel 1.25NO, CNC and CNC-PBA significantly improved the yield stress of hydrogels. Despite of different surface functionalities at $\mathrm{CNC}$ and CNC-PBA, the $1.25 / \mathrm{CNC} 2 \mathrm{NO}$ and $1.25 / \mathrm{CNC}$ PBA2NO had almost identical stress-strain curves before yielding points. However, after the yielding point, the CNC-PBA showed significantly greater reinforcement along the plastic deformation up to $1500 \%$ elongation. In contrast, $1.25 / \mathrm{CNC} 2 \mathrm{NO}$ required even slightly less stress during further elongation of up to $1500 \%$. In the static crosslinking networks, hydrogels 1.25/CNC2FO and 1.25/CNC-PBA2FO only showed elastic deformation. Interestingly, CNC acted as a better reinforcing nanofillers than CNC-PBA in these statically crosslinked hydrogels. This was probably attributed to that the extra PBA from CNC-PBA may have occupied more 
catechol groups and thus reduced the amount of dicatechol crosslinking.
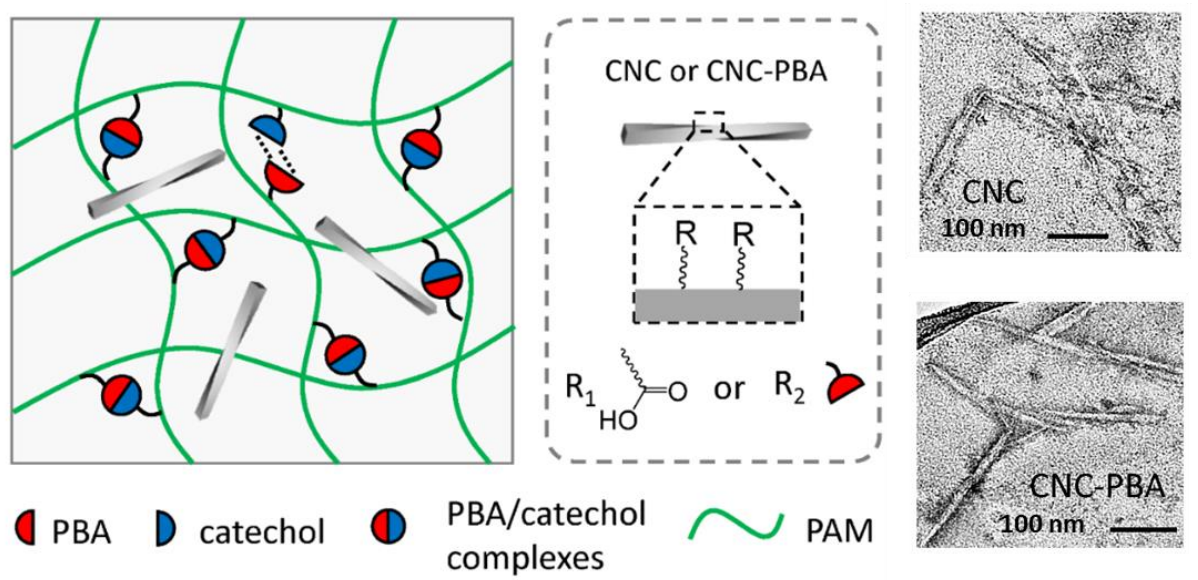

Neutral CNC:

With $\mathrm{COOH}$

Weak and nonspecific crosslinks

Active CNC-PBA:

With PBA

Strong and

specific

crosslinks

Figure 16. Schematic illustration for the composition of hydrogels and TEM images of CNC and CNC-PBA.

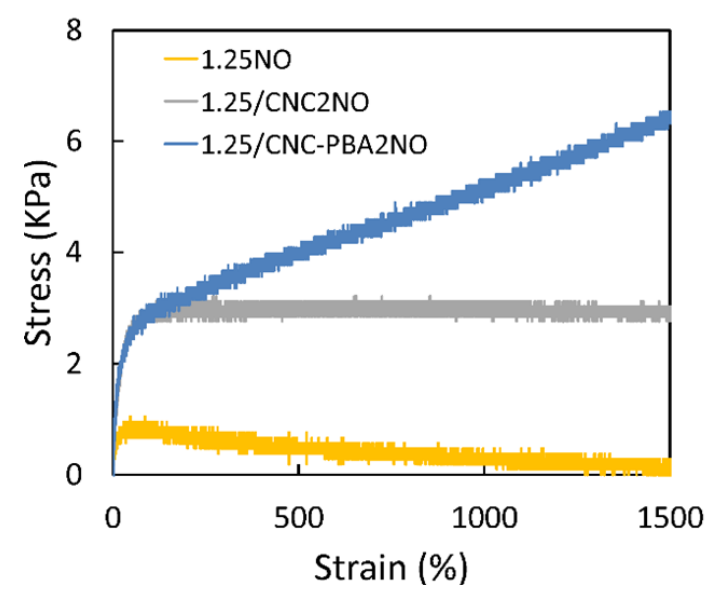

Non-oxidized Dynamic hydrogels CNC-PBA > CNC

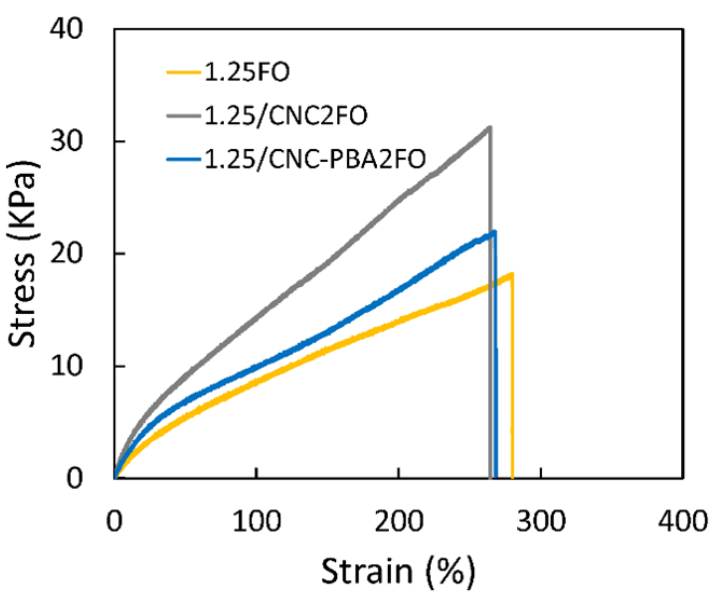

Oxidized Static hydrogels

CNC-PBA $<$ CNC

Figure 17. Contribution of CNC and CNC-PBA in dynamic hydrogels and static hydrogels.

In the top-oxidized hydrogel $1.25 \mathrm{TO}$, the plastic deformation of non-oxidized dynamic hydrogel dominated in the necking zones, while the PBA/catechol complexes continuously and rapidly dissociated and associated to maintain the physical integrity. Therefore, the active CNC-PBA was able to participate in the dynamic crosslinking networks and strengthened the necking zones (Figure 18). The elastic deformation mainly occurred in the non-necking zone, 
while CNC-PBA showed much less reinforcement in the elastic deformation, no matter in static or dynamic hydrogel matrix. This different performance of active CNC-PBA acting as an autonomous compensation mechanism narrowed the difference of mechanical stability between necking and non-necking zone, leading to retarded propagation of necking zone in whole hydrogels. In contrast, neutral CNC among the plastic deformation only had non-specific hydrogen bonds to surrounding polymer chains, so that it showed limited reinforcement in the plastic necking zone. However, neutral CNC effectively reinforced the static crosslinking network within the non-necking zone. Thus, in contrast to the active CNC-PBA, neutral CNC further amplified the mechanical difference between the necking and non-necking zones, and promoted the necking propagation.
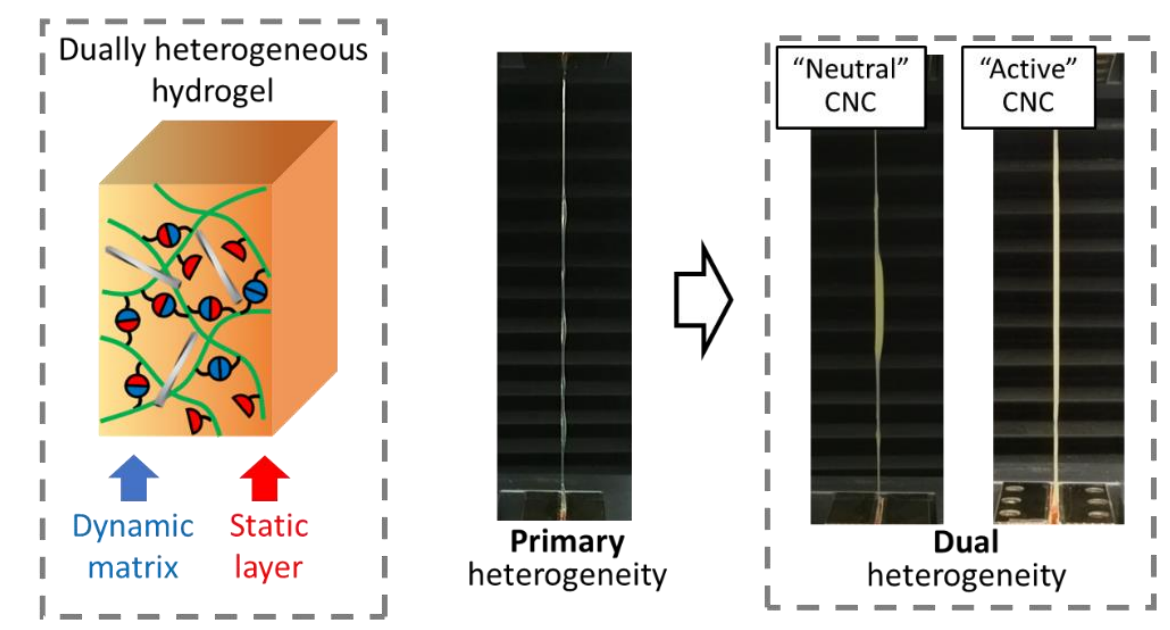

Necking zone (plastic deformation) Non-necking zone (elastic deformation)

CNC-PBA > CNC

CNC-PBA $<$ CNC

Figure 18. Tunable necking behaviors of dually heterogeneous hydrogels with $\mathrm{CNC}$ or CNCPBA.

On the basis of the results above, active CNC-PBA and neutral CNC performed various reinforcing mechanisms in hybrid hydrogels containing distinct cross-linking environments. Active CNC-PBA can participate in the dynamic cross-linking networks and strengthened the necking zones, but showed less reinforcement in non-necking zone. In contrast, neutral CNC only provided weak nonspecific hydrogen bonds in necking zone, but largely enhanced nonnecking zone. Therefore, diverse CNC regulated necking propagation in hybrid hydrogels. 
Because of these different working mechanisms of nanocrosslinkers in these hybrid crosslinking networks, the deformation and fracture mode of hydrogels were regulated, and effectively improved the physical integrity of hybrid hydrogels with uneven mechanical properties.

\subsection{Liquid behaviors-assisted material fabrication with dynamic hybrid hydrogels}

Liquid-solid transition is widely present in nature and artificial treatments as a useful strategy to mold polymers, metals or glasses. Rather than the formation of shapes, certain features of the liquid precursors are also utilized in the fabrication of advanced structural materials. For example, flowing liquids can align anisotropic particles toward the direction of shear stress. While the surface tension of static liquid system not only promotes the formation of specific shapes, but also influences the assembly behaviors of involatile components during the drying process. However, the static liquid properties only become dominant in the equilibrium state, while the flowing liquid is transient and highly dynamic. Therefore, their conflicting characters make it challenging to sequentially integrate the dynamic and static liquid behaviors in fabricating materials.

In the Publication 3, we demonstrated that CNC hybrid hydrogels crosslinked by dynamic covalent bonds, rather than classic liquid systems, can be used as precursor to sequentially integrate the dynamic liquid behaviors and static liquid behaviors in the same materials.

Because of the highly dynamic PBA/catechol crosslinking (binding constant $\mathrm{K}=0.919 \times 10^{3} \mathrm{M}^{-}$ ${ }^{1}$ ), the mechanical behaviors of hybrid dynamic hydrogels were sensitive to the frequency of external loading (Figure 19). In the static state, hydrogels DH1.25/CNC2 showed a liquid state with high complex viscosity, in which the crosslinking networks were slowly interchanged (Figure 20). 

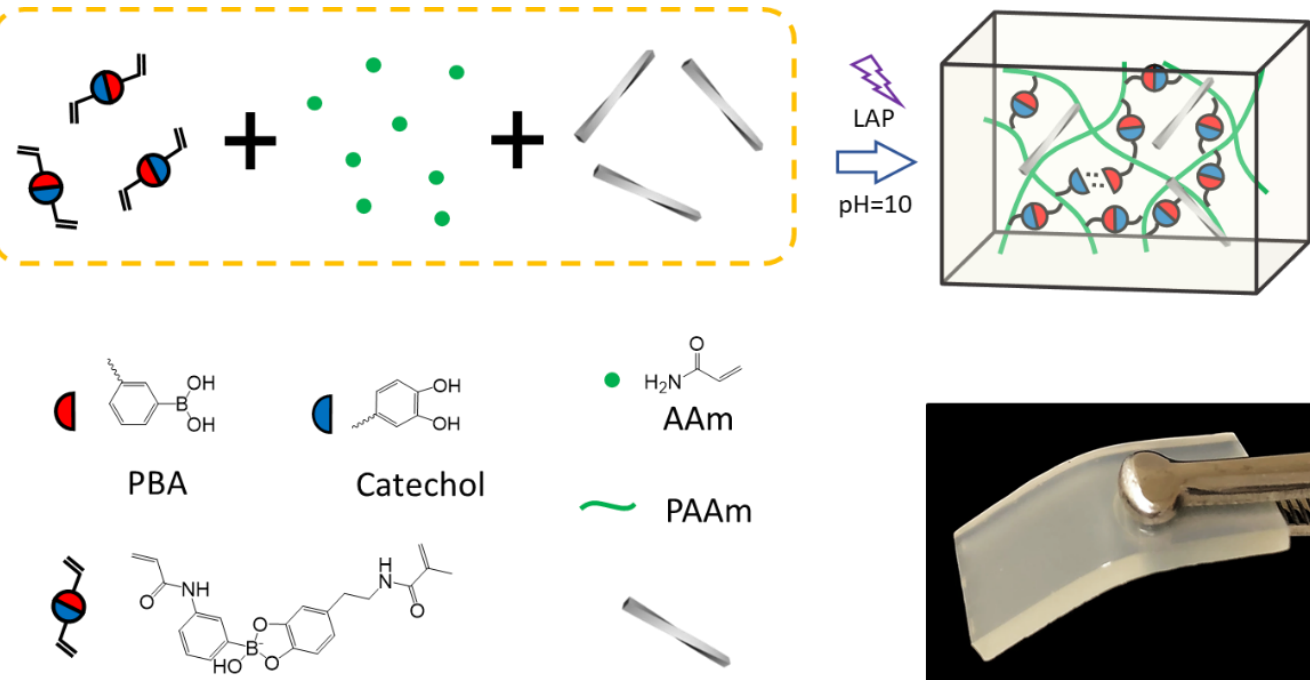

PBA/catechol dynamic crosslinker
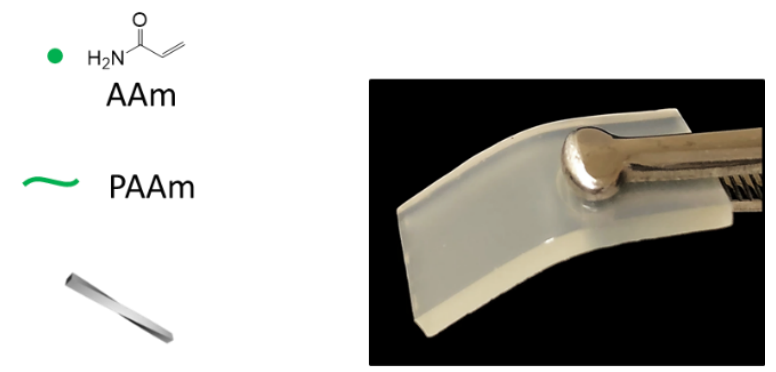

Figure 19. The composition of hybrid dynamic hydrogels with CNC.
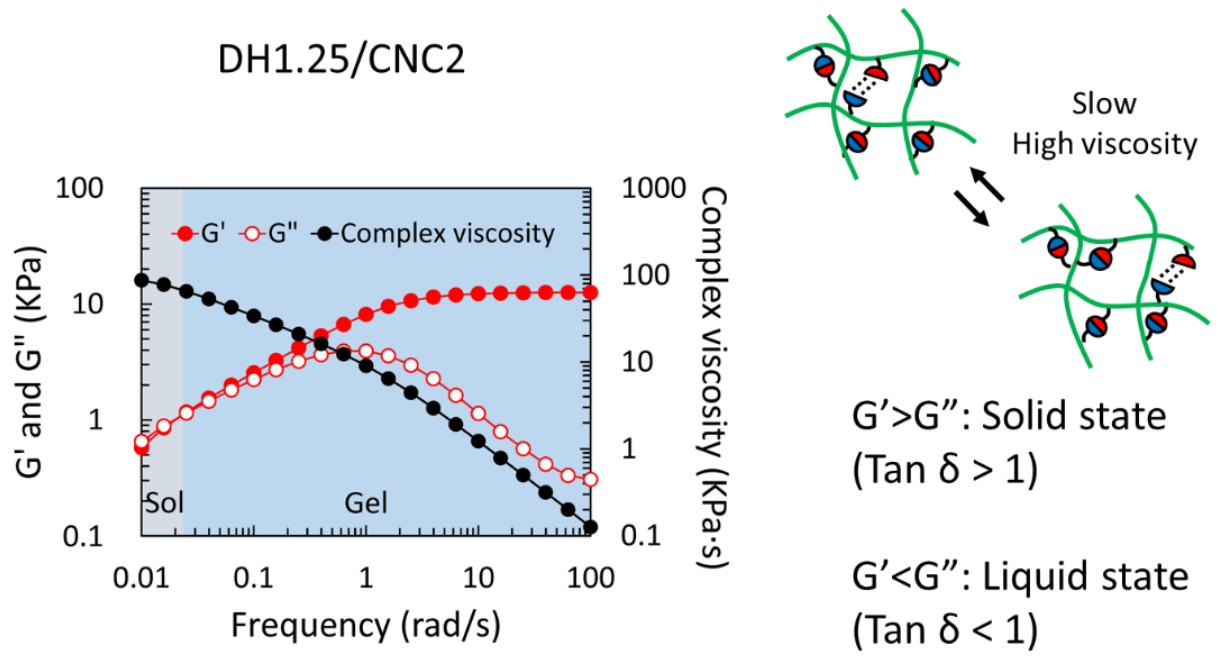

Figure 20. Static liquid properties of hybrid dynamic hydrogels.

Furthermore, another dynamic liquid state of $\mathrm{DH} 1.25 / \mathrm{CNC} 2$ was driven by violent shearing

(Figure 21). This shear thinning property was caused by the temporary dissociation of $\mathrm{PBA} /$ catechol complexes. Once the large strain was removed, these disrupted dynamic covalent bonds were fully reconstructed within 500 seconds.

Different to the dynamic covalent crosslinking in the molecular scale, $\mathrm{CNC}$ are nanoneedle structures with length in hundreds of nanometers. After the violent shearing loading, the 
arrangement of $\mathrm{CNC}$ in aqueous suspension cannot be fully relaxed within 500 seconds which led to the diminished complex viscosity (Figure 22). The slow relaxation of CNC arrangement was resisted by rapidly reconstructed hydrogels networks. Therefore, the footprint of hydrodynamic alignment of $\mathrm{CNC}$ was preserved in the rapidly relaxed hydrogel matrix.
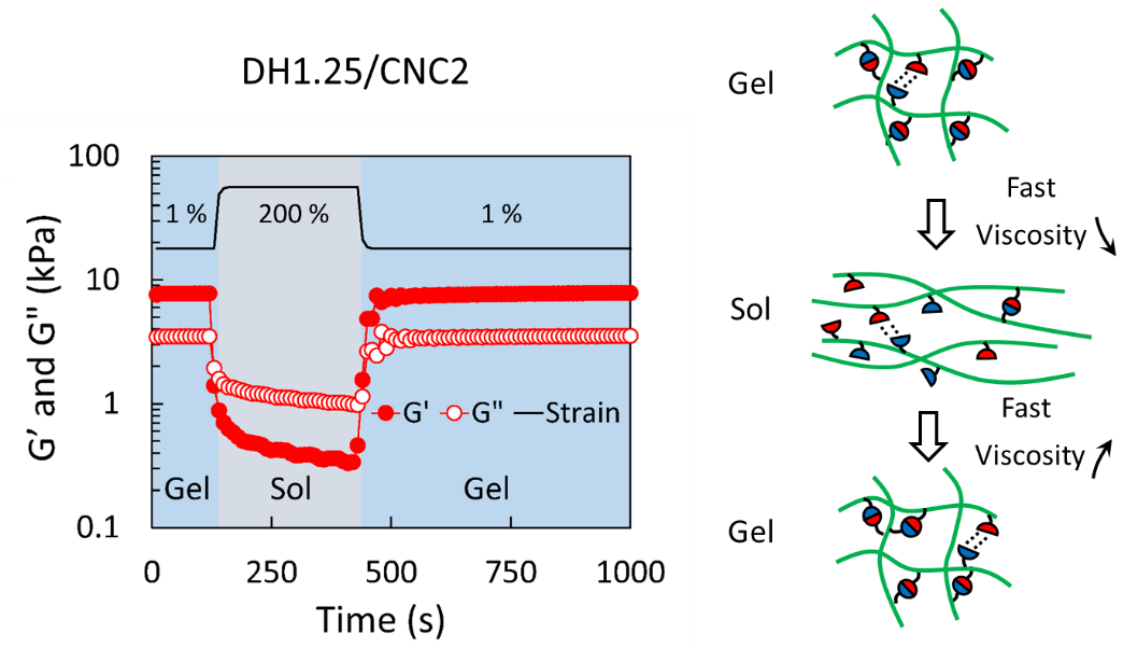

Figure 21. Liquid properties of hybrid dynamic hydrogel in the dynamic state.

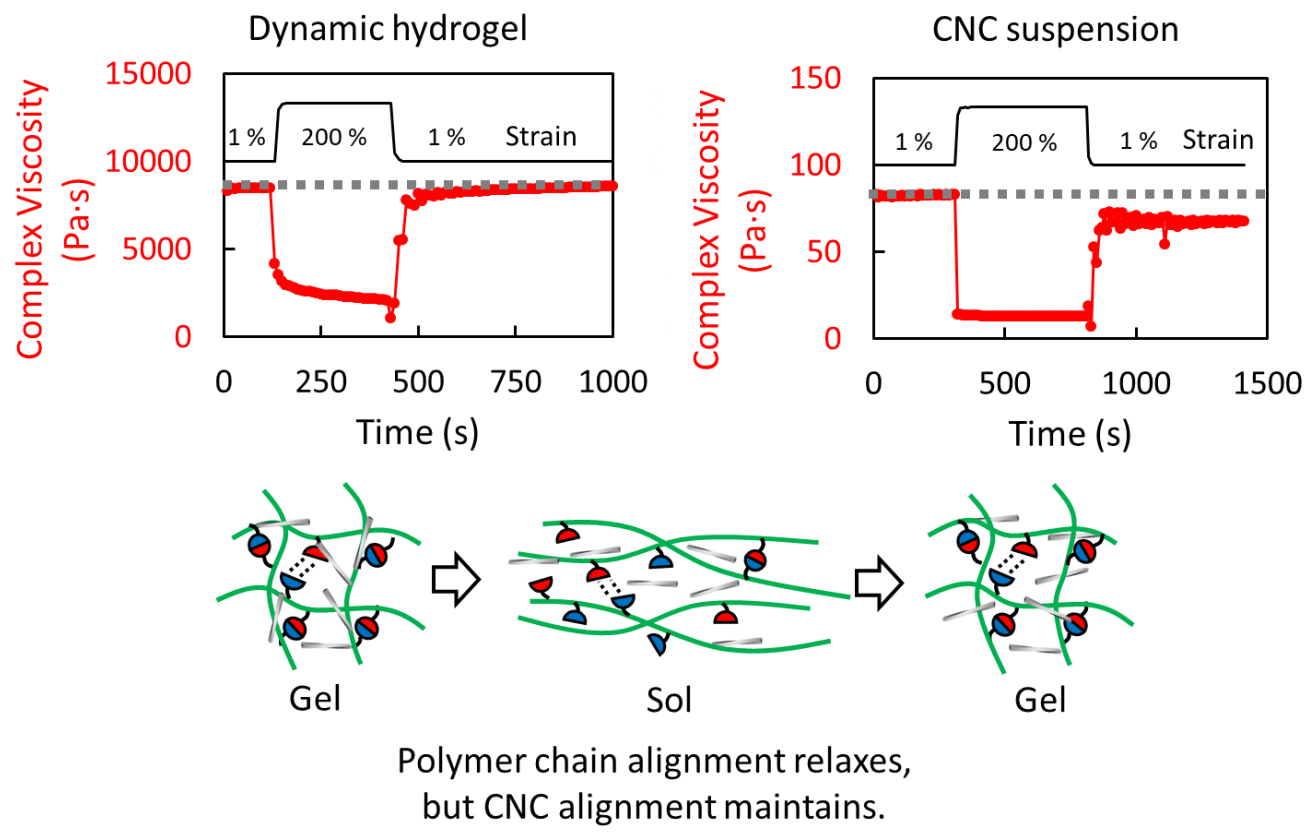

Figure 22. The alignment of $\mathrm{CNC}$ was fixed by the rapidly recovered dynamic hydrogel crosslinking networks.

During the uniaxial stretching, a continuous interference colors were observed in the 
DH1.25/CNC2. This phenomenon represented the aligned CNC in the hydrogels after the uniaxial stretching, which was driven by the dynamic liquid properties in the hybrid dynamic hydrogels (Figure 23). This orientation of CNC was further enhanced by drying the stretched dynamic hydrogels in air. After the air dry with constant elongation ratio, xerogel XG1.25/CNC2 demonstrated further amplified rainbow-like interference colors with continuous color distributions. These interference colors observed between cross polarizers were corresponded to retardations, which were accumulated phase differences between lights vibrating perpendicular and parallel to aligned $\mathrm{CNC}$.

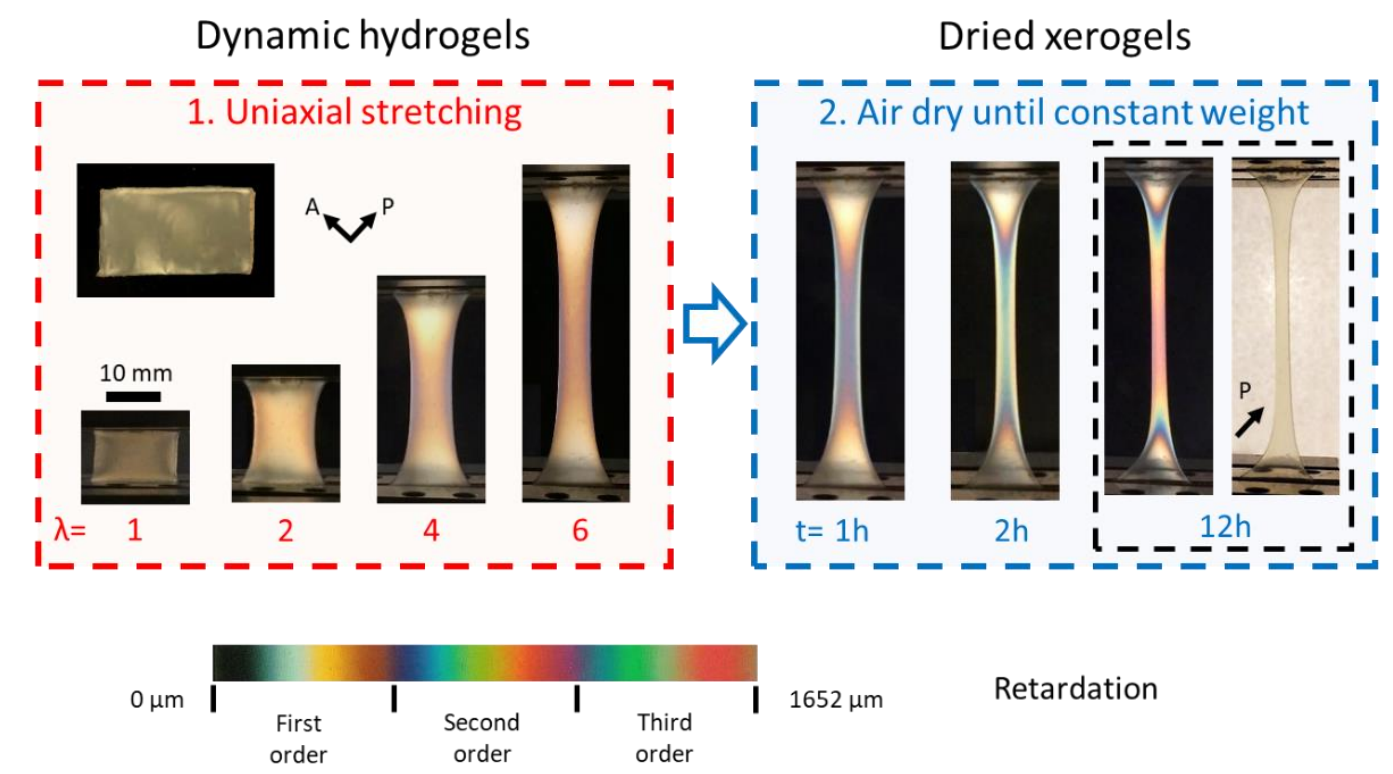

Figure 23. Polarizing images of $\mathrm{DH} 1.25 / \mathrm{CNC} 2$ during uniaxial stretching and air-drying.

Due to different alignment of $\mathrm{CNC}$ within contracted edges and the interior of xerogels, the geometry of dynamic hydrogel can be used to tune the contracted edges and thus to further control the orientation of $\mathrm{CNC}$ in the resulted xerogels. In Figure 24, a series of hydrogels DH1.25/CNC2 were prepared with various width/depth $\left(\mathrm{W}_{0} / \mathrm{D}_{0}\right)$ ratios between 1 and 32. For hydrogels with $\mathrm{W}_{0} / \mathrm{D}_{0}=1$, the corresponding xerogels have the greatest birefringence of $0.00414 \pm 0.00022$ due to the confinement of edges during the drying. With $\mathrm{W}_{0} / \mathrm{D}_{0}=32$, the gradient orientation of $\mathrm{CNC}$ was obtained at the edge of XG1.25/CNC2 (birefringence from 0.00210 to 0.00352 ). While a uniform film in the middle of the xerogel with birefringence of $0.00210 \pm 0.00012$ was obtained. 
a

Dynamic hydrogels $\quad W_{0} / D_{0}=12.5 \quad 5 \quad 7.5 \quad 12$

20

$20 \quad 32$
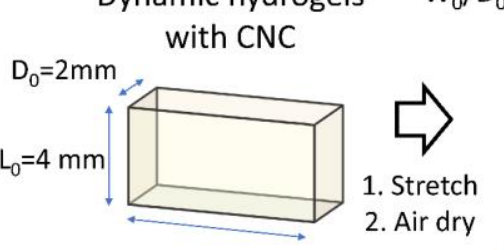

1. Stretch

2. Air dry

$\mathrm{W}_{0}$

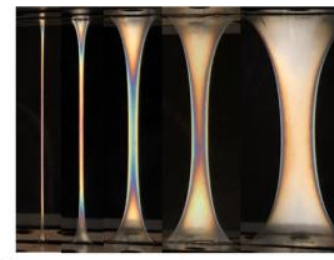

32

A $=10 \mathrm{~mm}$

b
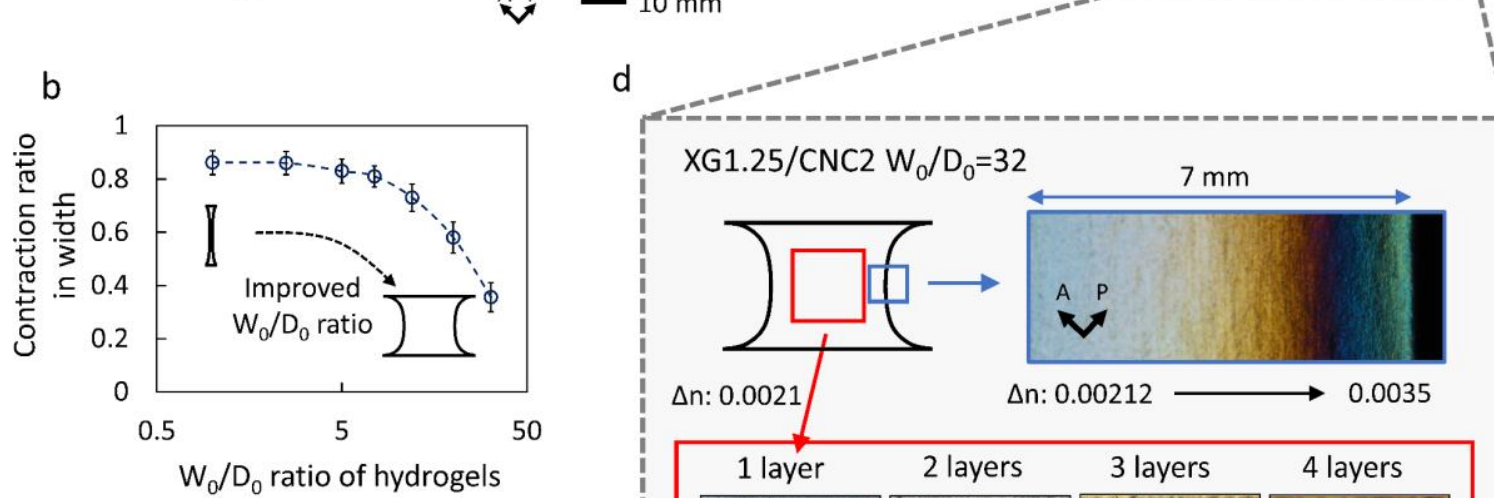

$X G 1.25 / C N C 2 W_{0} / D_{0}=32$
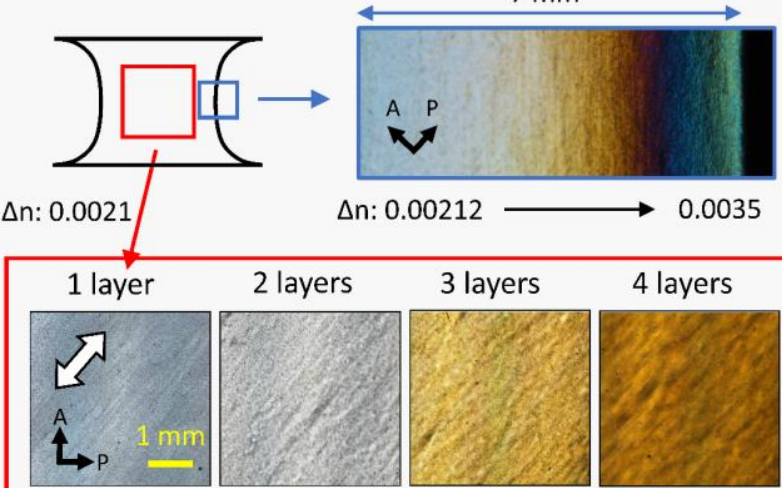

5 layers

6 layers

7 layers

8 layers
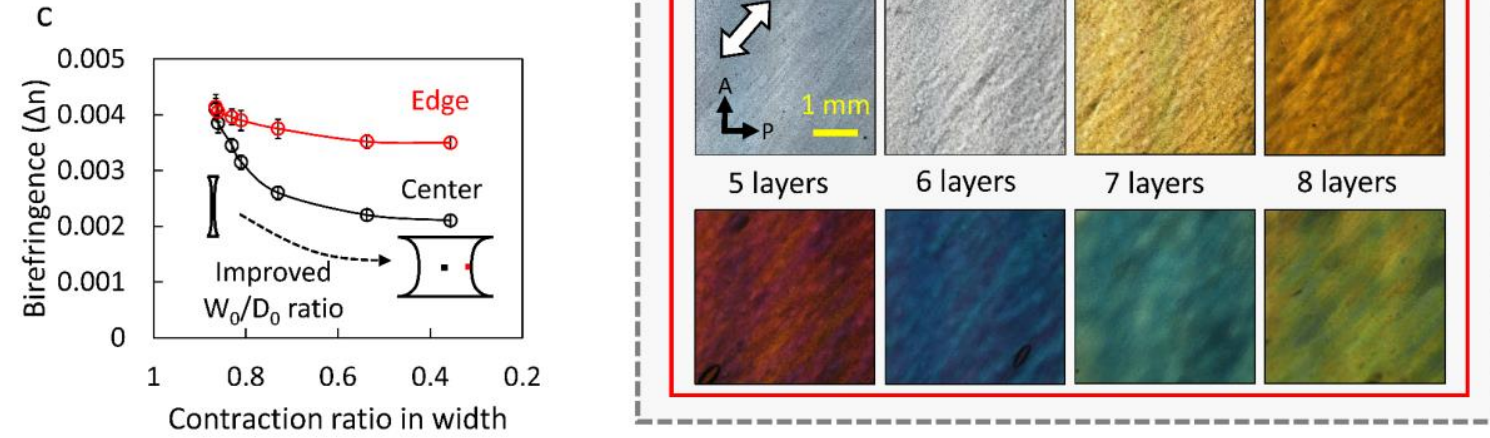

Figure 24. Preparation of $\mathrm{XG} 1.25 / \mathrm{CNC} 2$ with various width/depth ratio $\left(\mathrm{W}_{0} / \mathrm{D}_{0}\right)$

The shape of resulted xerogels can be encoded by the shape of dynamic hydrogels precursors.

In Figure 25, the xerogel fibers can be prepared with rectangular, triangular or round crosssection and with uniform or gradient interference colors. 
Dynamic hydrogels

a

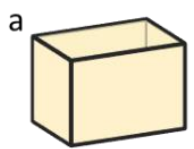

Rectangle

b

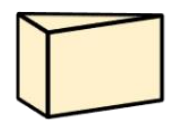

Triangle

C

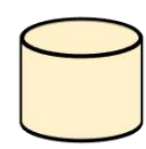

Round
Xerogels with programable shape
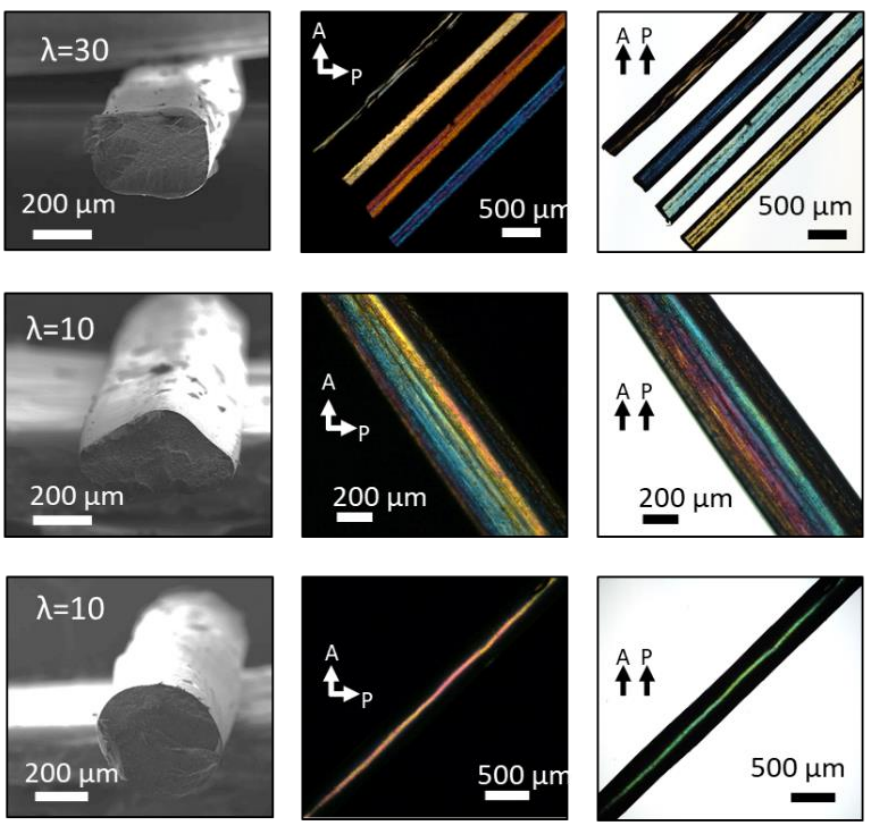

Figure 25. a) XG1.25/CNCx fibers with rectangular cross-section. The dark field polarizing images from left top to right bottom: $\mathrm{CNC}$ amounts $\mathrm{x}$ were $0.5,1,1.5$ and $2 \mathrm{wt} \%$ in the original dynamic hydrogels. b) XG1.25/CNC2 fibers with triangular cross-section. c) XG1.25/CNC2 fibers with round cross-section.

In addition, the sequential control of static and dynamic properties of dynamic hydrogels provided the flexibility to program spatial complex 3D shapes with well-ordered $\mathrm{CNC}$, which was difficult to achieve with flow-assisted method in classic liquid systems. In the present work, with aligned $\mathrm{CNC}$, the shape of stretched hydrogels can be reprogramed before the drying process (Figure 26). As examples, by rotating around a Teflon stick, stretched DH1.25/CNC2 can be turned into an elastic xerogel spring with interference colors. Moreover, twisted xerogels were prepared by rotating stretched $\mathrm{DH} 1.25 / \mathrm{CNC} 2$ axially before the drying process.

Hollow dynamic hydrogels have been investigated as a further model to prepare complex 3D dry polymers with curved surface (Figure 27). The tubular xerogels were obtained from dynamic hydrogel tubes, while a similar confinement of the hydrogel edges as those within films and fibers was not present. The drying hydrogel tubes were able to develop concave structures towards the center of tubes, which was driven by surface tension of hydrogels to 
pursue the minimal surface area. Following this, the hollow xerogels with pseudo catenoidmimetic morphology were generated.

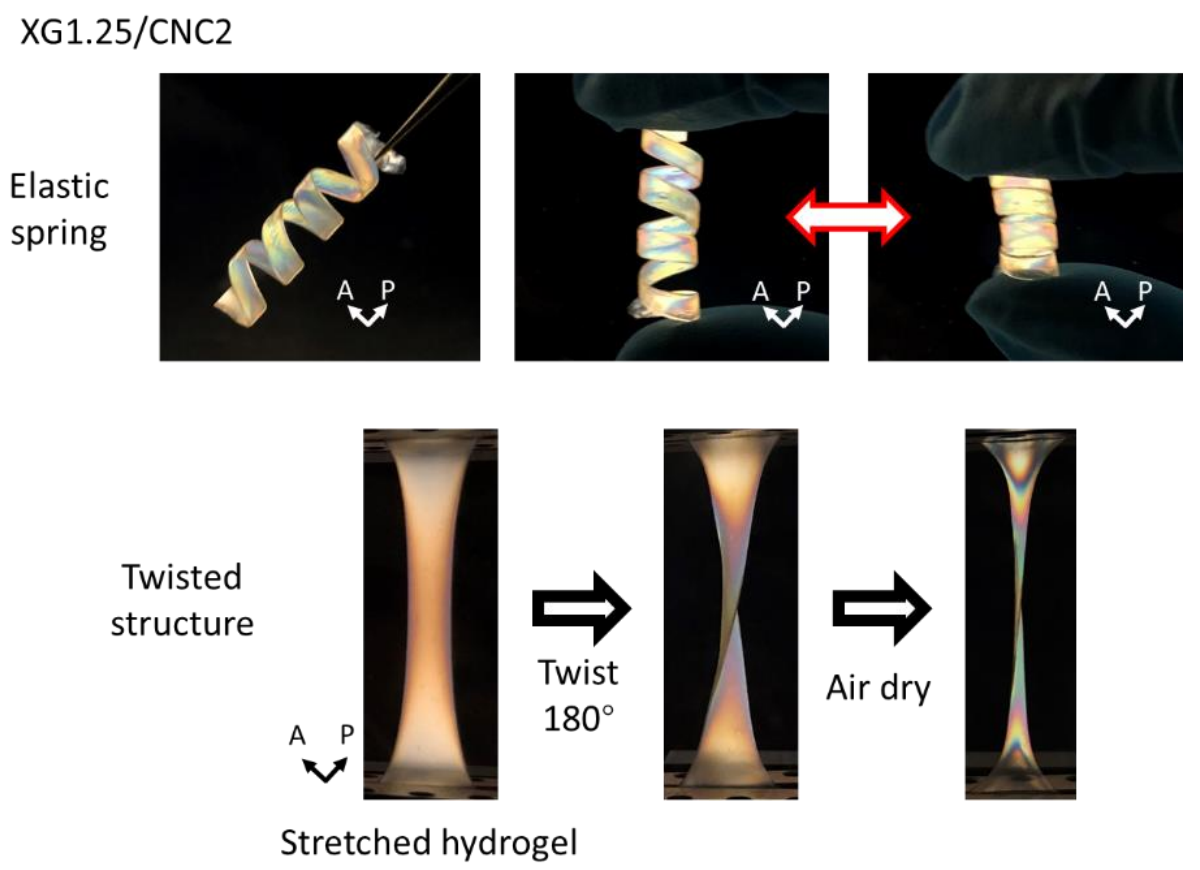

Figure 26. XG1.25/CNC2 as elastic spring or with twisted structure.

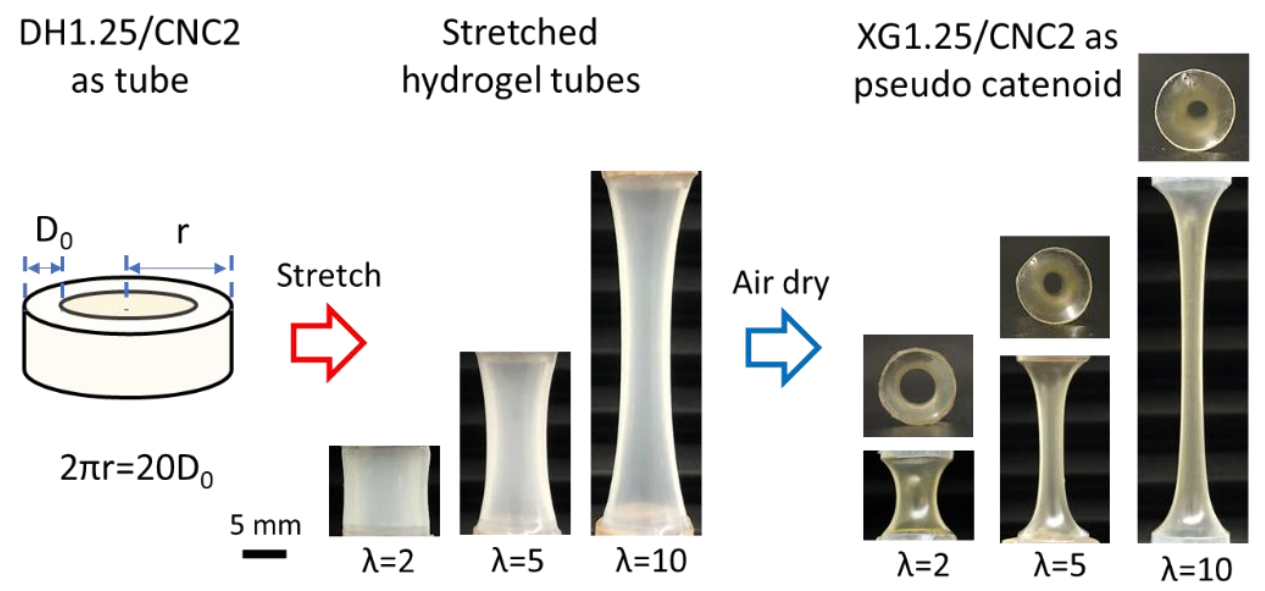

Figure 27. XG1.25/CNC2 as pseudo catenoid

In the present section, we demonstrated that the liquid-behaviors-assisted material fabrication can be expanded to 3D morphologies without the limitation of fluid channels, which was due to the liquid behaviors of dynamic hybrid hydrogels with CNC. During the uniaxial stretching, 
a transient liquid state was created and drove the alignment of anisotropic $\mathrm{CNC}$ in the dynamic hydrogels. Importantly, this $\mathrm{CNC}$ alignment was preserved by rapidly reconstructed hydrogel networks after the stretching. With subsequent drying in air, $\mathrm{CNC}$ alignment got further improved and generated highly tunable birefringence in resulting xerogels. Since dynamic hydrogels themselves provided focusing boundaries for liquid behaviors, the geometry of resulting xerogels can be versatile via remolding before the drying process.

In conclusion, the dynamic hydrogels containing anisotropic nanoparticles provide a new mold-free strategy to program multidimensional birefringent materials. With highly programmable geometry and tunable composition, these birefringent xerogels can be applied as diverse functional materials. As an example, fibrous xerogels can be used as a yarn to prepare tough but flexible textiles with hidden patterns. Furthermore, pseudo-catenoid xerogels can be prepared as lightweight engineering materials to acquire extreme load-bearing capability. 


\section{General conclusion and perspectives}

As mentioned all along this study, cellulose nanocrystals (CNC) and surface modified CNC were introduced in the crosslinking networks of hydrogels to create the dynamic heterogeneous hydrogels. Because of functional surface and excellent mechanical properties, CNC nanocrosslinkers could promote the mechanical properties of synthetic hydrogels. Furthermore, the anisotropic shape and unique optical properties of CNC provided the building block to construct multidimensional birefringent polymer materials, which was highly relying on the liquid properties of dynamic hydrogels with $\mathrm{CNC}$.

Firstly, in Publication 1, by mimicking the highly heterogeneous structure in the natural hydrogels, we prepared a novel kind of dynamic composite hydrogels with a heterogeneous structure based on the host-guest pre-organized dynamic CNC nano-crosslinkers. The crosslinking agents, diverse acrylated $\beta-\mathrm{CD}$, were immobilized on the $\mathrm{CNC}$ surface via preorganized host-guest interaction between $\beta-\mathrm{CD}$ and $\mathrm{ADA}$. These acrylated $\beta$-CD contributed to further construction of hydrogels via purely physical or hybrid chemical/physical interactions. Because of the highly heterogeneous structure, the conjunctions on the CNCpolymer interface exhibited gradual detachment under exerted stress and worked as an effective energy dissipation system. The sacrificed $\beta-\mathrm{CD} / \mathrm{ADA}$ conjunction showed a time-dependent recoverability in the as prepared hydrogels. However, this recoverability was lost when the hydrogels got fully swollen, even if they were still toughened by the same energy dissipation system. This phenomenon was due to the highly heterogeneous structure caused by swelling. Along with the volume increase, the uneven expansion of nano-crosslinkers and flexible polymers generated residual stress on the $\mathrm{CNC}$-polymer interface, and therefore resisted the recovery of the sacrificed $\beta-C D / A D A$ conjunctions.

In addition to improving mechanical properties, the heterogeneous toughening mechanism of hydrogels also significantly delayed the propagation of the crack. The crack propagation during the stretching of pre-notched hydrogels was paused and retarded until the complete breakup. This could be attributed to the dynamic host-guest bonding on the $\mathrm{CNC}$ surface. The sequential 
dissociation of the $\beta$-CD/ADA complexes and the heterogeneous hydrogel structures effectively retarded the crack propagation.

Then, in Publication 2, surface-functionalized CNC were utilized to regulate the necking phenomenon of hydrogels. A novel group of heterogeneous hydrogels was prepared by combining static top layer and dynamic matrix with distinct spatial distributions, which provided the primary heterogeneity in macrostructures. During the long-distance stretching of these heterogeneous hydrogels, the discontinuous ruptures of oxidized static layer led to necking deformation. This necking deformation did not occur in solely dynamically or statically crosslinked hydrogels. Furthermore, as releaser for the secondary heterogeneity, anisotropic nanosized $\mathrm{CNC}$ were introduced into the heterogeneous hydrogels. Active CNC with dynamic bonds (PBA) on surface could actively establish supramolecular interactions with other counter parts (catechol) within hydrogels, while neutral CNC with carboxyl groups could primarily form non-specific hydrogen bonds with other components within hydrogels. Consequently, the necking deformation and the mechanical properties of the dually heterogeneous hydrogels could be tuned by introducing diverse CNC. By fully utilizing the distinct reinforcing mechanisms of $\mathrm{CNC}$ with different surface-functionalities in various dynamic environments, we achieved a strategy to regulate the necking deformation in the hydrogel system and thus adjusted their mechanical stability.

Finally, in the publication 3, the liquid behaviors of dynamic heterogeneous hydrogels were applied to fabricate dry polymers with complexed shapes and highly ordered microstructures. In this work, we demonstrated that hybrid dynamic hydrogels with $\mathrm{CNC}$ can sequentially integrate the dynamic and static liquid behaviors to construct the same materials. In a longdistance uniaxial stretching, the shear thinning behaviors of dynamic hydrogels drove a hydrodynamic alignment of CNC. Since the reconstruction of hydrogel networks was much faster than the dissipation of CNC orientation, the alignment of CNC was maintained when the stretching was terminated. Therefore, the footprint of dynamic liquid behaviors was kept in the relaxed hydrogel matrix. Then, static liquid properties took effect: the surface tension of drying hydrogels further improved the CNC alignment and also programmed dried xerogels with diverse shapes from fibers to films and even complexed three dimensional structures. 
Thus, by utilizing liquid properties in dynamic hydrogels, the flowing assisted alignment of anisotropic particles can be easily manipulated in the 3D spaces and got rid of the limitation of the fluid channel. Furthermore, the alignment of particles can be maintained in the relaxed hydrogel networks and achieve the integrity of dynamic and static liquid behaviors in the fabrication of birefringent polymer materials.

In conclusion, $\mathrm{CNC}$ and surface modified $\mathrm{CNC}$ provided heterogeneous microstructures in the dynamic hydrogels. For the reinforcement of mechanical properties, well-designed dynamic CNC nanocrosslinkers effectively improved the mechanical toughness of hydrogels, and reduced the influence of local defect. Furthermore, the necking phenomenon of hydrogels can be controlled by the active $\mathrm{CNC}$ nanocrosslinker, which narrowed the mechanical difference of diverse hydrogel components and stabilized their shapes and mechanical behaviors as a hybrid. Besides the mechanical reinforcement, by exploiting the liquid behaviors in a dynamic hydrogel with $\mathrm{CNC}$, we can integrate the efficacies of both dynamic and static liquid behaviors to fabricate birefringent polymer materials. Thus, dynamic heterogeneous hydrogels can be used as precursors to achieve a mold-free methodology to program dry polymers with various $3 \mathrm{D}$ geometries and anisotropic microstructures.

According to the studies in this thesis, several superiorities of dynamic heterogeneous hydrogels have been uncovered, especially in the mechanical reinforcement and the fabrication of anisotropic polymeric materials. In the future, more efforts need to be done to fully illustrate the structure-performance relationship in the soft and hydrate hydrogel materials. Furthermore, in this study, even we mainly focus on the $\mathrm{CNC}$ with needle-like shape, but the mechanical toughening mechanism and material fabrication methods can be easily expanded to the nanostructures with diverse shapes and compositions, therefore provide a wide variety of practical applications in the future. 


\section{References}

1. Zhang, Y. S.; Khademhosseini, A. Science 2017, 356.

2. Danno, A. J. Phys. Soc. Jpn. 1958, 13, 722.

3. Buwalda, S. J.; Boere, K. W.; Dijkstra, P. J.; Feijen, J.; Vermonden, T.; Hennink, W. E. J. Control. Release 2014, 190, 254.

4. Thiele, J.; Ma, Y.; Bruekers, S. M.; Ma, S.; Huck, W. T. Adv. Mater. 2014, 26, 125.

5. Seliktar, D. Science 2012, 336, 1124.

6. Burdick, J. A.; Murphy, W. L. Nat. Commun. 2012, 3, 1269.

7. Ionov, L. Mater. Today 2014, 17, 494.

8. Appel, E. A.; Forster, R. A.; Rowland, M. J.; Scherman, O. A. Biomaterials 2014, 35, 9897.

9. Gaharwar, A. K.; Avery, R. K.; Assmann, A.; Paul, A.; McKinley, G. H.; Khademhosseini, A.; Olsen, B. D. ACS nano 2014, 8, 9833.

10. Zhang, K.; Feng, Q.; Xu, J.; Xu, X.; Tian, F.; Yeung, K. W. K.; Bian, L. Adv. Funct. Mater. 2017, 27, 1701642 .

11. Siqueira, G.; Kokkinis, D.; Libanori, R.; Hausmann, M. K.; Gladman, A. S.; Neels, A.; Tingaut, P.; Zimmermann, T.; Lewis, J. A.; Studart, A. R. Adv. Funct. Mater. 2017, 27, 1604619.

12. Zhao, X. Proc. Natl. Acad. Sci. U.S.A. 2017, 114, 8138.

13. Kamadaa, A.; Mittal, N.; Söderberg, L. D.; Ingverud, T.; Ohmd, W.; Roth, S. V.; Lundell, F.; Lendele, C. Proc. Natl. Acad. Sci. U.S.A. 2017, 114, 1232.

14. Zhao, X. Soft Matter 2014, 10, 672.

15. Zhong, M.; Wang, R.; Kawamoto, K.; Olsen, B. D.; Johnson, J. A. Science 2016, 353, 1264.

16. Sakai, T.; Matsunaga, T.; Yamanoto, Y.; Ito, C.; Yoshida, R.; Suzuki, S.; Sasaki, N.; Shibayama, M.; Chung, U. I. Macromolecules 2008, 41, 5379.

17. Kamata, H.; Akagi, Y.; Kayasuga-Kariya, Y.; Chung, U. I.; Sakai, T. Science 2014, 343, 873.

18. Gong, J. P.; Katsumata, Y.; Kurokawa, T.; Osada, Y. Adv. Mater. 2003, 15, 1155.

19. Gong, J. P. Soft Matter 2010, 6, 2583.

20. Okumura, Y.; Ito, K. Adv. Mater. 2001, 13, 485.

21. Imran, A. B.; Esaki, K.; Gotoh, H.; Seki, T.; Ito, K.; Sakai, Y.; Takeoka, Y. Nat. Commun. 
2014, 5,5124 .

22. Sun, T. L.; Kurokawa, T.; Kuroda, S.; Ihsan, A. B.; Akasaki, T.; Sato, K.; Haque, M. A.; Nakajima, T.; Gong, J. P. Nat. Mater. 2013, 12, 932.

23. Campbell, S. B.; Patenaude, M.; Hoare, T. Biomacromolecules 2013, 14, 644.

24. Huang, Y.; King, D. R.; Sun, T. L.; Nonoyama, T.; Kurokawa, T.; Nakajima, T.; Gong, J. P. Adv. Funct. Mater. 2017, 27, 1605350.

25. Illeperuma, W. R. K.; Sun, J.-Y.; Suo, Z.; Vlassak, J. J. Extreme Mechanics Letters 2014, 1, 90.

26. Zhang, W.; Liu, X.; Wang, J.; Tang, J.; Hu, J.; Lu, T.; Suo, Z. Eng. Fract. Mech. 2018, 187, 74.

27. Hu, X.; Vatankhah-Varnoosfaderani, M.; Zhou, J.; Li, Q.; Sheiko, S. S. Adv. Mater. 2015, 27,6899 .

28. Wei, K.; Zhu, M.; Sun, Y.; Xu, J.; Feng, Q.; Lin, S.; Wu, T.; Xu, J.; Tian, F.; XIa, J.; Li, G.; Bian, L. Macromolecules 2015, 49, 866.

29. Fan, H.; Wang, J.; Jin, Z. Macromolecules 2018, 51, 1696.

30. Hu, X.; Zhou, J.; Daniel, W. F. M.; Vatankhah-Varnoosfaderani, M.; Dobrynin, A. V.; Sheiko, S. S. Macromolecules 2017, 50, 652.

31. Chen, Q.; Zhu, L.; Zhao, C.; Wang, Q.; Zheng, J. Adv. Mater. 2013, 25, 4171.

32. Appel, E. A.; del Barrio, J.; Loh, X. J.; Scherman, O. A. Chem. Soc. Rev. 2012, 41, 6195.

33. Grindy, S. C.; Learsch, R.; Mozhdehi, D.; Cheng, J.; Barrett, D. G.; Guan, Z.; Messersmith, P. B.; Holten-Andersen, N. Nat. Mater. 2015, 14, 1210.

34. Kakuta, T.; Takashima, Y.; Nakahata, M.; Otsubo, M.; Yamaguchi, H.; Harada, A. Adv. Mater. 2013, 25, 2849.

35. Ihsan, A. B.; Sun, T. L.; Kuroda, S.; Haque, M. A.; Kurokawa, T.; Nakajima, T.; Gong, J. P. J. Mater. Chem. B 2013, 1, 4555.

36. Luo, F.; Sun, T. L.; Nakajima, T.; Kurokawa, T.; Zhao, Y.; Sato, K.; Ihsan, A. B.; Li, X.; Guo, H.; Gong, J. P. Adv. Mater. 2015, 27, 2722.

37. Hunt, J. N.; Feldman, K. E.; Lynd, N. A.; Deek, J.; Campos, L. M.; Spruell, J. M.; Hernandez, B. M.; Kramer, E. J.; Hawker, C. J. Adv. Mater. 2011, 23, 2327. 
38. Lv, S.; Dudek, D. M.; Cao, Y.; Balamurali, M. M.; Gosline, J.; Li, H. Nature 2010, 465, 69.

39. Yang, X.; Bakaic, E.; Hoare, T.; Cranston, E. D. Biomacromolecules 2013, 14, 4447.

40. Guan, Y.; Zhang, Y. Chem. Soc. Rev. 2013, 42, 8106.

41. Yesilyurt, V.; Webber, M. J.; Appel, E. A.; Godwin, C.; Langer, R.; Anderson, D. G. Adv. Mater. 2016, 28, 86.

42. Yang, J.; Zhang, X.; Ma, M.; Xu, F. ACS Macro Lett. 2015, 4, 829.

43. Lopez Hernandez, H.; Grosskopf, A. K.; Stapleton, L. M.; Agmon, G.; Appel, E. A. Macromol. Biosci. 2018, e1800275.

44. Zhang, S.; Greenfield, M. A.; Mata, A.; Palmer, L. C.; Bitton, R.; Mantei, J. R.; Aparicio, C.; de la Cruz, M. O.; Stupp, S. I. Nat. Mater. 2010, 9, 594.

45. Caliari, S. R.; Burdick, J. A. Nat. Methods 2016, 13, 405.

46. Rosales, A. M.; Anseth, K. S. Nat. Rev. Mater. 2016, 1, 15012.

47. Brown, T. E.; Anseth, K. S. Chem. Soc. Rev. 2017, 46, 6532.

48. Gao, G.; Du, G.; Sun, Y.; Fu, J. ACS Appl. Mater. Interfaces 2015, 7, 5029.

49. Liu, M.; Ishida, Y.; Ebina, Y.; Sasaki, T.; Aida, T. Nat. Commun. 2013, 4, 2029.

50. Takashima, Y.; Hatanaka, S.; Otsubo, M.; Nakahata, M.; Kakuta, T.; Hashidzume, A.; Yamaguchi, H.; Harada, A. Nat. Commun. 2012, 3, 1270.

51. Nakahata, M.; Mori, S.; Takashima, Y.; Hashidzume, A.; Yamaguchi, H.; Harada, A. ACS Macro Lett. 2014, 3, 337.

52. Takashima, Y.; Yonekura, K.; Koyanagi, K.; Iwaso, K.; Nakahata, M.; Yamaguchi, H.; Harada, A. Macromolecules 2017, 50, 4144.

53. Harada, A.; Takashima, Y.; Nakahata, M. Accounts of chemical research 2014, 47, 2128.

54. Liu, L.; Ghaemi, A.; Gekle, S.; Agarwal, S. Adv. Mater. 2016, 28, 9792.

55. Nakamura, T.; Takashima, Y.; Hashidzume, A.; Yamaguchi, H.; Harada, A. Nat. Commun. 2014, 5,4622 .

56. Harada, A.; Kobayashi, R.; Takashima, Y.; Hashidzume, A.; Yamaguchi, H. Nat. Chem. 2011, 3,34 .

57. Iwaso, K.; Takashima, Y.; Harada, A. Nat. Chem. 2016, 8, 625.

58. Lee, B. P.; Konst, S. Adv. Mater. 2014, 26, 3415. 
59. Mann, J. L.; Yu, A. C.; Agmon, G.; Appel, E. A. Biomater. Sci. 2017, 6, 10.

60. Yuk, H.; Lin, S.; Ma, C.; Takaffoli, M.; Fang, N. X.; Zhao, X. Nat. Commun. 2017, 8, 14230.

61. Webber, R. E.; Creton, C.; Brown, H. R.; Gong, J. P. Macromolecules 2007, 40, 2919.

62. Thoniyot, P.; Tan, M. J.; Karim, A. A.; Young, D. J.; Loh, X. J. Adv. Sci. 2015, 2, 1400010.

63. Wang, Q.; Gao, Z. J. Mech. Phys. Solids 2016, 94, 127.

64. Yang, J.; Han, C.-R.; Duan, J.-F.; Ma, M.-G.; Zhang, X.-M.; Xu, F.; Sun, R.-C. Cellulose 2013, 20, 227.

65. Appel, E. A.; Tibbitt, M. W.; Webber, M. J.; Mattix, B. A.; Veiseh, O.; Langer, R. Nat. Commun. 2015, 6, 6295.

66. Hu, Z.; Chen, G. Adv. Mater. 2014, 26, 5950.

67. Li, Q.; Barrett, D. G.; Messersmith, P. B.; Holten-Andersen, N. ACS nano 2016, 10, 1317.

68. Wang, Q.; Mynar, J. L.; Yoshida, M.; Lee, E.; Lee, M.; Okuro, K.; Kinbara, K.; Aida, T. Nature 2010, 463, 339.

69. Haraguchi, K.; Uyama, K.; Tanimoto, H. Macromol. Rapid Commun. 2011, 32, 1253.

70. Wu, Y.; Shah, D. U.; Liu, C.; Yu, Z.; Liu, J.; Ren, X.; Rowland, M. J.; Abell, C.; Ramage, M. H.; Scherman, O. A. Proc. Natl. Acad. Sci. U. S. A. 2017, 114, 8163.

71. Pekař, M. Front. Mater. 2015, 1, 35.

72. Marcelo, G.; López-González, M.; Mendicuti, F.; Tarazona, M. P.; Valiente, M. Macromolecules 2014, 47, 6028.

73. Habibi, Y. Chem. Soc. Rev. 2014, 43, 1519.

74. Zimmermann, T.; Pöhler, E.; Geiger, T. Adv. Eng. Mater. 2004, 6, 754.

75. Almeida, A. P. C.; Canejo, J. P.; Fernandes, S. N.; Echeverria, C.; Almeida, P. L.; Godinho, M. H. Adv. Mater. 2018, 30, e1703655.

76. Parker, R. M.; Guidetti, G.; Williams, C. A.; Zhao, T.; Narkevicius, A.; Vignolini, S.; FrkaPetesic, B. Adv. Mater. 2018, 30, e1704477.

77. Mittal, N.; Ansari, F.; Gowda, V. K.; Brouzet, C.; Chen, P.; Larsson, P. T.; Roth, S. V.; Lundell, F.; Wagberg, L.; Kotov, N. A.; Soderberg, L. D. ACS nano 2018, 12, 6378.

78. Hakansson, K. M.; Fall, A. B.; Lundell, F.; Yu, S.; Krywka, C.; Roth, S. V.; Santoro, G.; Kvick, M.; Prahl Wittberg, L.; Wagberg, L.; Soderberg, L. D. Nat. Commun. 2014, 5, 4018. 
79. McKee, J. R.; Hietala, S.; Seitsonen, J.; Laine, J.; Kontturi, E.; Ikkala, O. ACS Macro Lett. 2014, 3, 266.

80. McKee, J. R.; Appel, E. A.; Seitsonen, J.; Kontturi, E.; Scherman, O. A.; Ikkala, O. Adv. Funct. Mater. 2014, 24, 2706.

81. Shao, C.; Wang, M.; Chang, H.; Xu, F.; Yang, J. ACS Sustain. Chem. Eng. 2017, 5, 6167.

82. Asthana, R.; Kumar, A.; Dahotre, N., B. Materials Processing and Manufacturing Science; Elsevier, 2006.

83. Studart, A. R. Chem. Soc. Rev. 2016, 45, 359.

84. Kim, J.; Peretti, J.; Lahlil, K.; Boilot, J. P.; Gacoin, T. Adv. Mater. 2013, 25, 3295.

85. Ragelle, H.; Tibbitt, M. W.; Wu, S. Y.; Castillo, M. A.; Cheng, G. Z.; Gangadharan, S. P.; Anderson, D. G.; Cima, M. J.; Langer, R. Nat. Commun. 2018, 9, 1184.

86. Lan, Y.; Caciagli, A.; Guidetti, G.; Yu, Z.; Liu, J.; Johansen, V. E.; Kamp, M.; Abell, C.; Vignolini, S.; Scherman, O. A.; Eiser, E. Nat. Commun. 2018, 9, 3614.

87. Parker, R. M.; Frka-Petesic, B.; Guidetti, G.; Kamita, G.; Consani, G.; Abell, C.; Vignolini, S. ACS nano 2016, 10, 8443 .

88. Yao, K.; Meng, Q.; Bulone, V.; Zhou, Q. Adv. Mater. 2017, 29, 1701323.

89. Trebbin, M.; Steinhauser, D.; Perlich, J.; Buffet, A.; Roth, S. V.; Zimmermann, W.; Thiele, J.; Förster, S. Proc. Natl. Acad. Sci. U.S.A. 2013, 111, 6706.

90. Gladman, A. S.; Matsumoto, E. A.; Nuzzo, R. G.; Mahadevan, L.; Lewis, J. A. Nat. Mater. 2016, $15,413$. 


\section{Appendix}

Author's peer-reviewed publications 


\section{Publication 1}

\section{Robust heterogeneous hydrogels with dynamic nanocrystals-polymer interface}

Heqin Huang ${ }^{1}$, Yonggui Wang ${ }^{1}$, Xiaojie Wang ${ }^{1}$, Florian Rehfeldt ${ }^{2}$ and Kai Zhang ${ }^{*}, 1$

${ }^{1}$ Wood Technology and Wood Chemistry, Georg-August-University of Göttingen, Büsgenweg 4, D-37077 Göttingen, Germany

${ }^{2}$ Third Institute of Physics - Biophysics, Faculty of Physics, Georg-August-University of Göttingen, Friedrich-Hund-Platz 1, D-37077 Göttingen, Germany

* Tel.: +49 551 394505; E-mail: kzhang1@uni-goettingen.de

Originally published in:

Macromolecular Rapid Communications

John Wiley \& Sons

DOI: $10.1002 /$ marc.201600810

Received: December 27, 2016; Revised: February 17, 2017; Published online: April 4, 2017 
Abstract: We designed a kind of novel heterogeneous composite hydrogels with dynamic nano-crosslinkers, which was built via the pre-organized host-guest interaction on the surface of cellulose nanocrystals. The reversible $\beta$-cyclodextrin/adamantane conjunctions and their gradual dissociation on the nanocrystals-polymer interface guaranteed the compressibility and stretchability of the composite hydrogels. While the sacrificed toughening mechanism could be rebuilt in the as prepared hydrogels, it failed to be regenerated in the swollen hydrogels. This fact was originally due to the extreme mechanical contrast between rigid nanocrystals and the flexible polymer phase. This heterogeneity was largely amplified by swelling process: polymer chains were pre-stretched between nano-crosslinkers and generated residual stress on the dynamic nanocrystals-polymer interface. Thus, this swelling-induced heterogeneity resisted the reassociation of the sacrificed $\beta$-cyclodextrin/adamantane complexes. Furthermore, the unstable nanocrystals-polymer interface induced the crack propagate along the nanocrosslinker surface, which remarkably retarded the crack propagation during the stretch.

Keywords: composite hydrogel, heterogeneous structure, cellulose nanocrystals, host-guest interaction, nanoparticle-polymer interface

\section{Introduction}

Hydrogels as highly hydrated polymeric materials are promising platforms for diverse applications, including tissue engineering, drug delivery systems and soft devices. ${ }^{[1]}$ However, traditional synthetic hydrogels are generally mechanically weak and brittle. This shortcoming has been partially overcome by several toughening strategies. ${ }^{[2]}$ One of the most important strategy was implementing a sacrificed energy dissipation systems in hydrogels. For instance, double network hydrogels have been widely investigated because of their extraordinary toughness and stretchability. ${ }^{[3,4]}$ The dense and brittle first network generated an effective energy dissipating system, while the dilute and flexible second network guaranteed the physical integrity. ${ }^{[5,6]}$ Rather than the disposable sacrificed bonding, many reversible energy dissipating mechanisms have also been utilized to resist fatigue in cyclic deformation: such as electrostatic interactions, ${ }^{[7]}$ metal ion-ligand coordination, ${ }^{[8]}$ hydrogen bonding, ${ }^{[9]}$ host-guest interactions, ${ }^{[10]}$ and well-designed transition of polymer domains. ${ }^{[11]}$ 
Hydrogels using multi-functionality crosslinkers provided another energy dissipation system: the polymer chains between these crosslinkers often have various lengths, and the sequentially detachment of polymer chains dissipated stored energy. ${ }^{[12]}$ According to this mechanism, composite hydrogels were also prepared with nano-crosslinkers, which were able to gather even more polymer chains around single crosslinking points. ${ }^{[13]}$ The polymer grafted on the nano-crosslinkers have a large range of length distribution, therefore the polymer chains could detach gradually and toughen the composite hydrogels. ${ }^{[14]}$ Except hydrogels covalently crosslinked by nano-crosslinkers, a few other hydrogel systems were synthesized based on the physical bonding between nanoparticles and polymer, such as electrostatic interactions ${ }^{[15]}$ and hydrophobic effect. ${ }^{[16]}$ Combining the advantages of nano-crosslinkers and reversible bonding, these dynamic nanocomposite hydrogels were often extremely tough and stretchable. Recently, scientists discussed the dynamic interface between $\mathrm{Fe}_{3} \mathrm{O}_{4}$ nanoparticles and catechol terminated poly(ethylene glycol) within hydrogel, and isolated the contributions of nanocrosslinkers and dynamic bonding. ${ }^{[17]}$ These studies provided valuable information about dynamic bonding on the nanoparticle-polymer interface, however, their behaviors in the swollen hydrogels were still largely unknown, especially during the mechanical deformation.

Cellulose nanocrystals (CNC) were mechanically strong nano-needles with native crystalline structures, ${ }^{[18]}$ which have been widely used in the composite hydrogels. But for most of the cases, $\mathrm{CNC}$ were integrated into the crosslinked polymer matrix ${ }^{[19]}$ or acted as covalent nanocrosslinkers. $^{[20]}$ Recently, through the electrostatic interaction between CNC and methylcellulose, a weak but thermo-responsive hydrogel has been prepared. ${ }^{[21]}$ In addition, modified CNC were also applied as dynamic nano-crosslinkers to form reinforced composite hydrogel. ${ }^{[22]}$ However, the dynamic bonding was settled in the polymer brush around $\mathrm{CNC}$, rather than on the rigid/soft interface, so that the influence of CNC-polymer interface was not shown directly.

In the present work, a novel type of heterogeneous composite hydrogel was prepared with surface-modified CNC as nano-crosslinkers. Acrylate groups were not directly grafted on the surface of the nano-crosslinkers, but were introduced via the host-guest interaction, and a pre- 
organization approach guaranteed a high bonding efficiency of $\beta$-cyclodextrin/adamantane ( $\beta$ $\mathrm{CD} / \mathrm{ADA}$ ) conjunctions. The dynamic multi-functionality nano-crosslinkers polymerized with acrylamide and formed composite hydrogels. The autonomous $\beta$-CD/ADA interaction and their sequentially dissociation on $\mathrm{CNC}$ nano-crosslinkers dissipated the elastic energy stored in the hydrogels, leading to robust compressibility and stretchability. Furthermore, the reversible nature of $\beta-\mathrm{CD} / \mathrm{ADA}$ complexes formed a recoverable toughening mechanism in the as prepared hydrogels. However, this toughening mechanism could not be regenerated within the swollen hydrogels. This difference should be attributed to the residual stress on the nanocrosslinker/polymer interface. The residual stress was generated by the stress concentration during swelling process, and originally because of the highly heterogeneous microstructure. Furthermore, because of the heterogeneous hydrogel structure and the dynamic nanocrystalspolymer interface, the crack propagation in the pre-notched hydrogels was also significantly retarded.

\section{Results and discussion}

\section{Fabrication of dynamic composite hydrogels with heterogeneous structures}

As shown in Figure. 1, anisotropic CNC were used as the hard and crystalline region, while polyacrylamide (PAAm) was used as the soft and amorphous part. These two components were connected via pre-organized host-guest conjunctions between $\beta-\mathrm{CD}$ and $\mathrm{ADA}$. CNC nanocrosslinkers not only acted as dynamic crosslinkers, but also gathered high density of polymers around them and provided heterogeneity. The pre-organization approach achieved a better bonding efficiency between $\beta-\mathrm{CD}$ and ADA. 


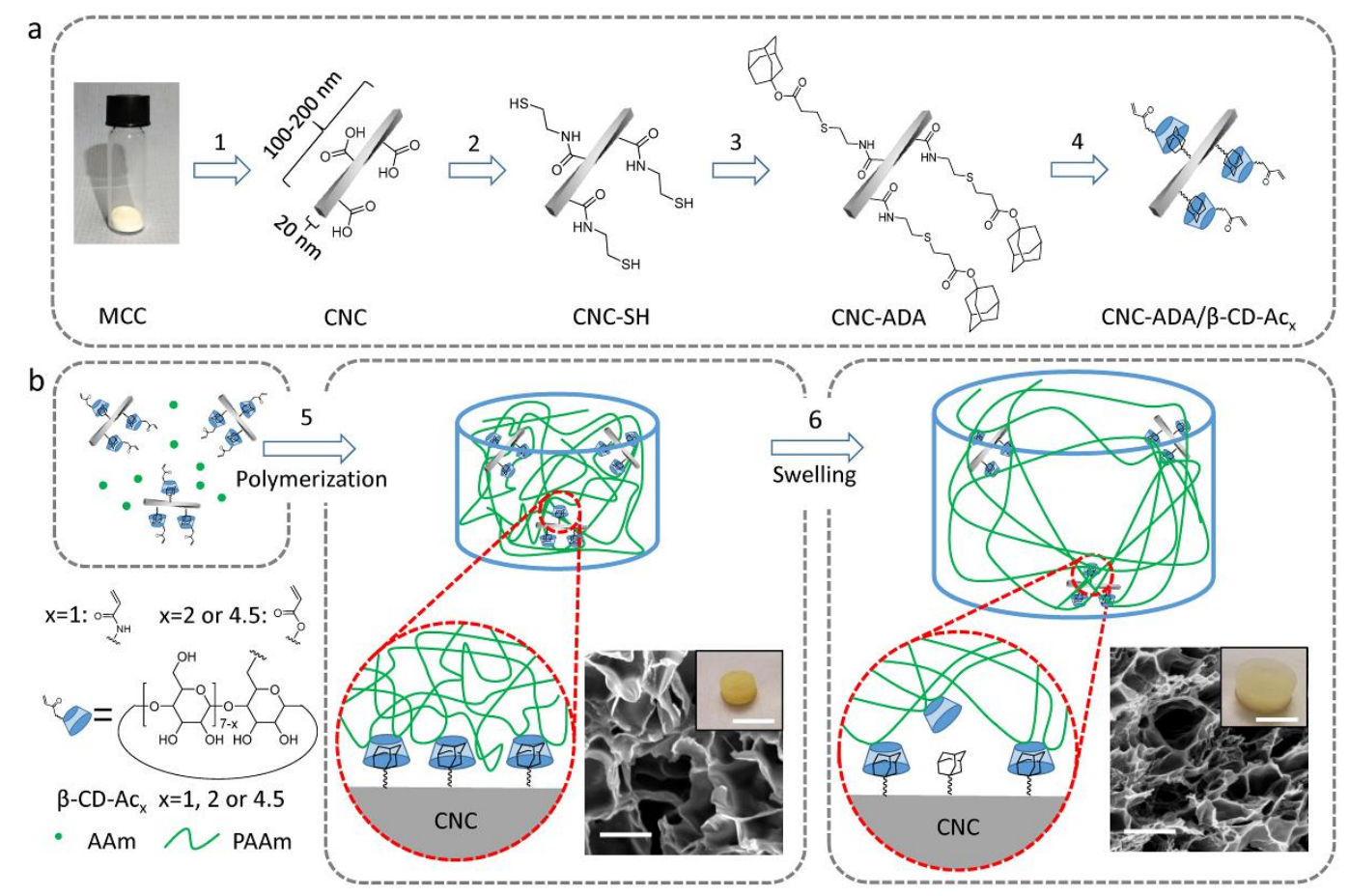

Figure 1. Schematic illustration for the preparation of dynamic composite hydrogels with preorganized nano-crosslinkers. a) Preparation of pre-organized CNC-ADA/ $\beta-C D-A c_{x}$ as the dynamic nano-crosslinkers. b) Preparation of dynamic composite hydrogels with the in situ polymerization. SEM images (scale bar: $2 \mu \mathrm{m}$ ) show the freeze-dried as prepared and fully swollen hydrogel DCH4/20/2. The insets show corresponding photographs before freezedrying (scale bar: $10 \mathrm{~mm}$ ).

CNC with carboxyl group content of $1.52 \mathrm{mmol} / \mathrm{g}$ were prepared from microcrystalline cellulose (MCC) via TEMPO-mediated oxidation (step 1 in Figure 1a). Via amidation reaction with cysteamine, thiol groups were introduced to CNC surface (step 2 in Figure 1a). Then, ADA was grafted on CNC-thiol (CNC-SH) via thiol-ene click-reaction, leading to $\mathrm{CNC}$ adamantane (CNC-ADA) (step 3 in Figure 1a). The ADA density on CNC-ADA was measured to be $0.67 \mathrm{mmol} / \mathrm{g}$. Therefore the nanocrystals with guest molecular were prepared (Figure S1 to S5).

Then, dynamic nano-crosslinkers were prepared via host-guest self-assembly of acrylate $\beta$ cyclodextrin $\left(\beta-C D-A c_{x}\right)$ and CNC-ADA in water (step 4 in Figure 1a). Three kinds of $\beta$-CD$A c_{x}$ were utilized with substitution ratio $x$ as 1.0, 2.0 and 4.5 (Figure S6-S9), which were named 
as $\beta-\mathrm{CD}-\mathrm{Ac}_{1}, \beta-\mathrm{CD}-\mathrm{Ac}_{2}$ and $\beta-\mathrm{CD}-\mathrm{Ac}_{4.5}$, respectively. The pre-organized nano-crosslinkers $\left(\mathrm{CNC}-\mathrm{ADA} / \beta-\mathrm{CD}-\mathrm{Ac}_{\mathrm{x}}\right.$ complexes) were separated from free $\beta-\mathrm{CD}-\mathrm{Ac} \mathrm{c}_{\mathrm{x}}$, and mixed with acrylamide (AAm) to form dynamic composite hydrogels by in situ polymerization (step 5 in Figure $1 \mathrm{~b}$ and Figure S10). The as prepared samples were immersed in water until no weight change (step 6 in Figure 1b) to get fully swollen hydrogels. All the representative recipes for the hydrogels used could be found in Table S1. For example, "DCH4/20/2" meant dynamic composite hydrogels prepared with $4 \mathrm{wt} \%$ of CNC-ADA, $20 \mathrm{wt} \%$ AAm and $\beta$-CD-Ac2. The scanning electron microscope (SEM) images of freeze-dried hydrogels in Figure $1 \mathrm{~b}$ showed the microstructure of as prepared and fully swollen DCH4/20/2 hydrogels, the insets reflected the volume change after the swelling. It was notable that the freeze-dried fully swollen hydrogels clearly showed a fibrous secondary structure compared to as prepared ones.

In order to understand the specialty of the heterogeneous structure and the effect of the dynamic interface to the hydrogels, in the following parts, detailed investigation was performed with both as prepared and fully swollen hydrogels.

\section{Investigation of mechanical properties}

As our hydrogels contained a heterogeneous structure with dynamic conjunctions on nanocrystals, the components would influence the overall mechanical features. To demonstrate the superiority of the approach for the construction of hydrogels and to isolate the contribution of each component, DCH4/20/1 was prepared and compared with its control groups (Figure 2a). $\mathrm{CNC}-\mathrm{ADA} / \mathrm{\beta}-\mathrm{CD}-\mathrm{Ac}_{1}$ complexes as non-covalent nano-crosslinkers formed a series of self-standing hydrogels after the polymerization with AAm, which showed a significant frequency dependency (Figure $2 \mathrm{~b}$ ). To exhibit the necessity of pre-organization approach, a control group 4/20/1-post was prepared: poly(AAm- $\beta-C D)$ was made in advance and then mixed with CNC-ADA suspension. Even with exactly the same component to DCH/4/20/1, resulted 4/20/1-post formed a kind of fluidic mixtures rather than hydrogel (Figure 2a, middle row). To isolate the contribution of CNC-ADA conjunctions, another control group 4/20(no $\beta$ CD) was prepared as well. Without polymerizable groups on the CNC surface, a viscous polymer colloid was obtained, and the large damping factors tan (delta) indicated the liquid 
features at a broad low frequency range (Figure $2 b$ and $2 c$ ).

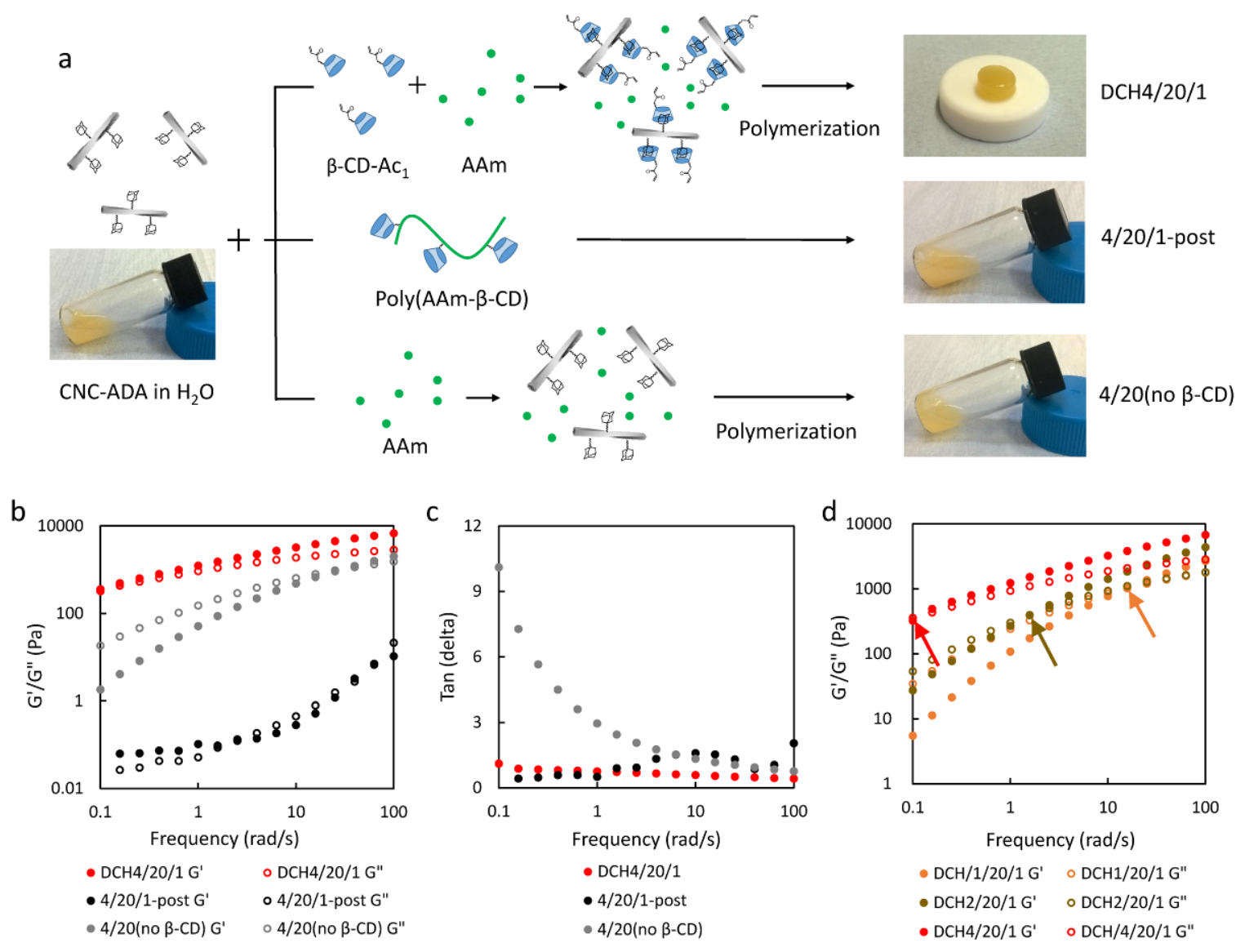

Figure 2. Preparation and properties of the dynamic composite hydrogels using pure physical nano-crosslinkers. (a) Schematic illustration for the preparation of the dynamic composite hydrogels with pre-organization approach (upper row), with the post-mix approach (middle row) and with in situ polymerization without $\beta-\mathrm{CD} / \mathrm{ADA}$ interaction (lower row). Rheology frequency sweeps of (b) storage modulus (G') and loss modulus (G”) as well as (c) the damping factors tan (delta) of dynamic composite hydrogels. (d) The dose dependency of nanocrosslinkers in the dynamic composite hydrogels. The arrows indicated the crossover points of G' and G'.

The dose dependency of the dynamic nano-crosslinkers was further studied by macro rheology. As shown in Figure 2d, the storage modulus ( $\left.G^{\prime}\right)$ of hydrogels was correlated to the amount of pre-organized nano-crosslinkers. A larger amount of nano-crosslinkers also made hydrogels 
more stable, which was reflected by the crossover point of G' and loss modulus (G') at even smaller frequency (pointed out by the arrows in Figure 2d). Moreover, the amount of the monomer also affected the mechanical properties of dynamic composite hydrogels (Figure S11a). The sample DCH4/5/1 aggregated already during the polymerization, because the extremely low polymer content could not form a continuous hydrogel network. As shown in the rheology frequency sweeps of DCH4/10/1 and DCH4/20/1 (Figure S11b), larger polymer amounts increased G' and made composite hydrogels more frequency-sensitive. Therefore, according to our results, $\beta-\mathrm{CD} / \mathrm{ADA}$ conjunctions effectively stabilized the heterogeneous structure within the hydrogels. At the same time, the pre-organization approach maximized the utility of such dynamic physical bonding.

Rather than the mono-substituted $\beta-\mathrm{CD}-\mathrm{Ac}_{1}$ providing pure physical dynamic interactions, multi-substituted $\beta$-CD-Ac2 and $\beta$-CD-Ac4.5 formed chemical/physical hybrid crosslinking networks around CNC. Compared with DCH/4/20/1, DCH/4/20/2 and DCH/4/20/4.5 showed only a negligible frequency dependency (Figure 3a). It should be noticed that the overall amount of acrylate groups on the CNC surface was similar (as shown in Table S1), even though different $\beta-C D-A c_{x}$ were used. This phenomenon was due to the various amounts of $\beta-C D-A c_{x}$ grafted on CNC-ADA. Therefore, the covalent crosslinking network strongly stabilized the hydrogel networks. In addition, three specimens showed similar G' at high frequencies, because where $\beta-\mathrm{CD} / \mathrm{ADA}$ conjunctions showed more similar behavior as covalent (Figure 3a). Rather than mechanical properties, the microstructure of hydrogels was also different. According to the SEM images of freeze-dried hydrogels (Figure 3b), DCH/4/20/1 exhibited pore diameter $\sim 1 \mu \mathrm{m}$ and the pore diameter of DCH4/20/2 increased to $\sim 3 \mu \mathrm{m}$, while DCH/4/20/4.5 had pore diameter as small as $\sim 200 \mathrm{~nm}$. 

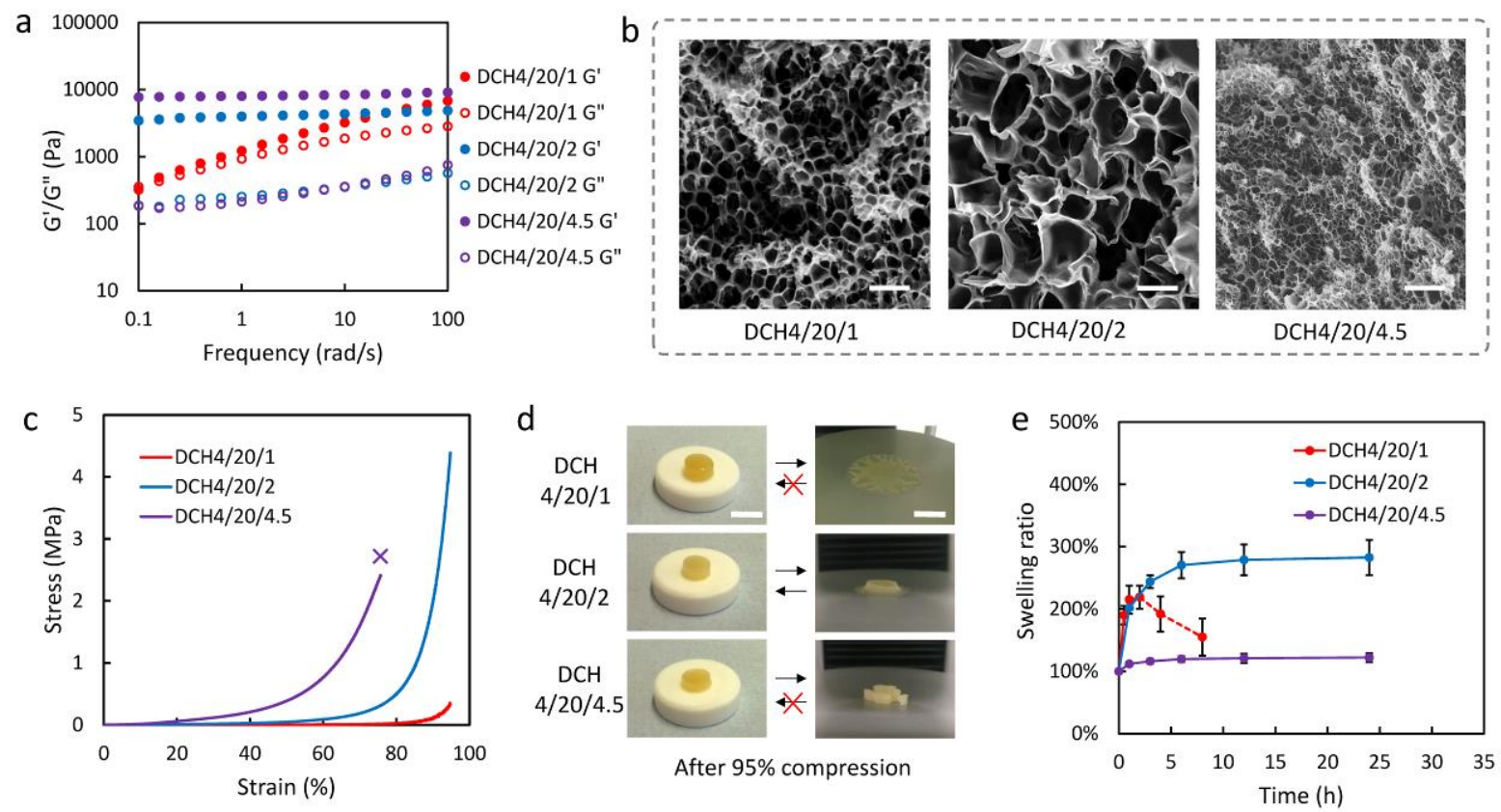

Figure 3. Properties of the dynamic composite hydrogels using hybrid physical/chemical nanocrosslinkers. (a) Frequency sweeps of DCH4/20/1, DCH4/20/2 and DCH4/20/4.5. (b) SEM images of freeze-dried hydrogels (scale bars: $2 \mu \mathrm{m}$, hydrogels were frozen in liquid nitrogen). (c) Compression strain-stress curves of dynamic composite hydrogels. The compression tests with $\mathrm{DCH} / 4 / 20 / 1$ and $\mathrm{DCH} / 4 / 20 / 2$ were terminated when the compression ratio reached $95 \%$. (d) The photographs (scale bars: $10 \mathrm{~cm}$ ) of hydrogels before and after the compression $(95 \%$ compression ratio). (e) Swelling ratios of hydrogels. The dash line for DCH4/20/1 reflected the residual weights.

In addition to the rheology measurements, the compression tests provided further valuable information about the mechanical properties. As shown in Figure 3c, DCH4/20/1 and DCH4/20/2 showed robust compressibility, and no obvious stress drop was observed up to $95 \%$ compression ratio. As the comparison, DCH4/20/4.5 showed much larger elastic modulus (indicated by the slope of initial strain-stress curve) and broken at the compression ratio of $\sim 75 \%$. Even DCH4/20/1 had no sudden drop in the compression stress. However, the compressed DCH4/20/1 could not return to its original shape (Figure 3d), which indicated its plastic nature. This plasticity have been reported by McKee et. al. in the $\mathrm{CNC} /$ methylcellulose composite hydrogel. ${ }^{[21]}$ Here in the present research, the $\beta-\mathrm{CD} / \mathrm{ADA}$ complexes as a stronger 
non-covalent bonding significantly reinforced the hydrogels, but still maintained the plasticity. The hydrogels DCH4/20/2 were adhered to the aluminum compression plate after the compression because of the large expansion in the horizontal direction. Once these adhered hydrogels were taken off from the compression plate, they were able to recover to their original shapes immediately. Moreover, the compression strain-stress curve of DCH4/20/2 could fully recover (Figure S12). The 95\% compression tests were repeatedly performed with the same specimen, and the strain-stress curves almost followed the virgin compression pathway (with interval longer than 15 min between each compression).

To further illustrate the toughening mechanism of our hydrogels, continuous hysteresis compression tests were carried out. To reduce the adhesion of hydrogels to the compression plate, the compression ratio of hydrogels was set as $60 \%$. Moreover, the compression tests were performed with as prepared specimens to avoid the volume change caused by swelling. As shown in Figure S13, the hysteresis in the second compression cycle was smaller than the first cycle for all three kinds of hydrogels DCH4/20/1, DCH4/20/2 and DCH4/20/4.5. The largest change of hysteresis was clearly notable for DCH4/20/1, while DCH4/20/4.5 showed the smallest one. The difference should be attributed to the different amounts of $\beta-\mathrm{CD} / \mathrm{ADA}$ conjunctions in the hydrogels and how much of them dissociated during the deformation. During the continuous compression tests, the sacrificed toughening mechanism did not have enough time to fully recover. Therefore, the change of hysteresis was visible. In comparison, hydrogels $\beta$-CD-Ac2/PAAm (similar recipe as $\mathrm{DCH} 4 / 20 / 2$, but only without $\mathrm{CNC}-\mathrm{ADA}$ ) showed almost the same pathway in three continuous compression cycles (Figure S13). This behavior was typical for the hydrogels without sacrificed toughening mechanisms. ${ }^{[5]}$ Furthermore, the second and the third hysteresis were identical for all the hydrogel specimens, indicating that no more sacrificed bonding was consumed without further deformation.

For hydrogels, the water content plays an important role in mechanical properties, and hydrogels are often used as fully swollen status. ${ }^{[23]}$ Figure 3 e showed the swelling behaviors of dynamic composite hydrogels. Because of the dense microstructure and multi-functionality $\beta$ CD-Ac4.5, the hydrogels DCH4/20/4.5 showed a very small swelling ratio (about 120\%). In 
comparison, DCH4/20/2 could swell to $280 \%$ of their original weights. The purely physically crosslinked hydrogels DCH4/20/1, however, were not stable in water for a long time (Figure S14). The swelling ratio of DCH4/20/1 in Figure 3e only reflected the residual weights of hydrogels during the swelling.

It is well understood that swelling is a pre-stretch process for polymer chain in hydrogels. ${ }^{[23]}$ For hydrogels with the heterogeneous structure via nano-crosslinkers, this pre-stretch would generate concentrated stress on the nanoparticle-polymer interface. In our heterogeneous dynamic hydrogels, this rigid-soft interface was connected by the autonomous physical hostguest interaction. This interaction was driven away from equilibrium status and more likely to dissociate, therefore made the hydrogels less stable under the stress exerted by swelling.

In order to understand this influence of swelling, compression and tensile tests were further performed. We compared the recoverability of toughening mechanism between as prepared and fully swollen dynamic composite hydrogels. Because the hydrogels DCH4/20/2 showed relatively large swelling ratios and still kept the physical integrity, they were selected for this investigation. Figure 4a showed the repeated compression curves of as prepared DCH4/20/2. Without intervals between each compression cycle, the second hysteresis loop became smaller and narrower. This sacrificed system could be recovered after a $15 \mathrm{~min}$ interval. In the tensile tests of hydrogels DCH4/20/2, similar behaviors were noticed as well, but they needed longer time for the recovery. DCH4/20/2 were firstly stretched to 6 times of its original length. The virgin tensile strain-stress curve could be fully recovered after $12 \mathrm{~h}$ interval, which indicated the recoverability of the sacrificed mechanism. 

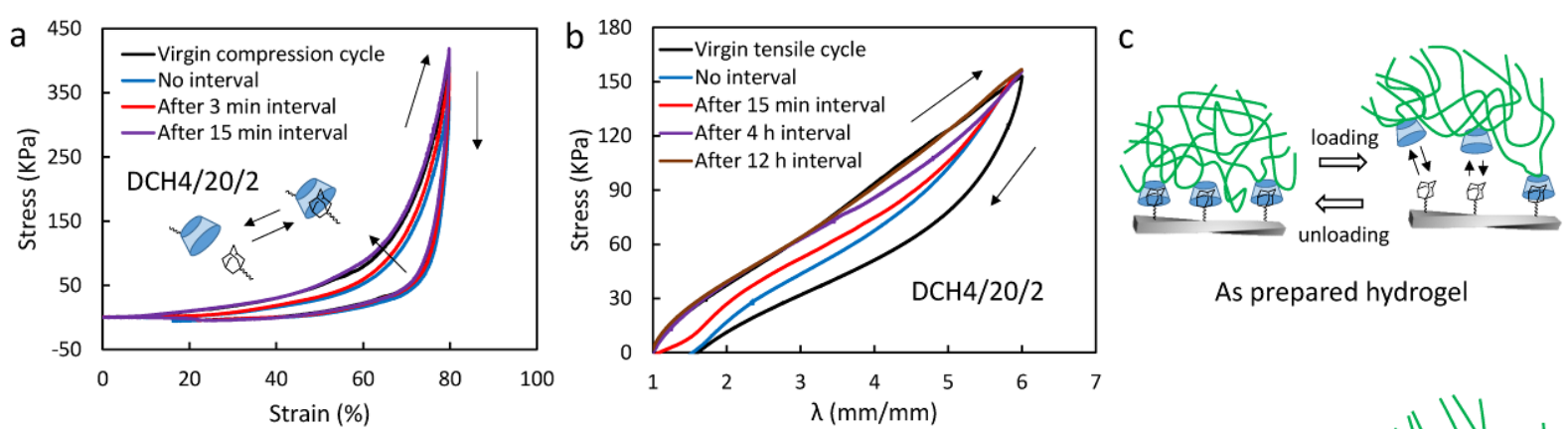

As prepared hydrogel
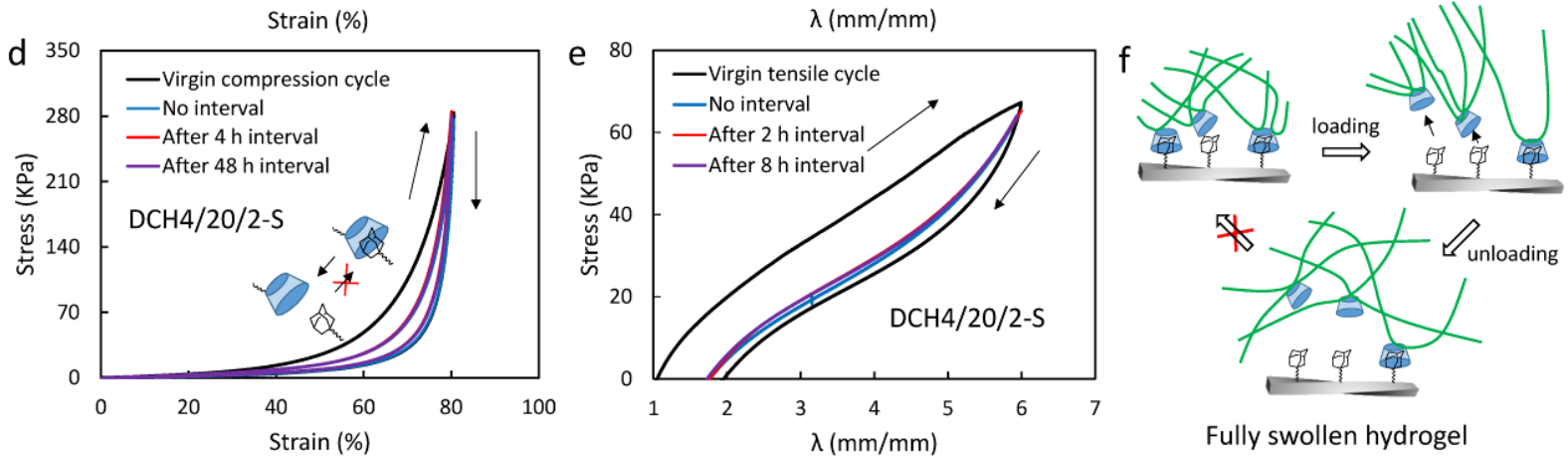

Figure 4. Mechanical properties of as prepared and fully swollen hydrogels. The (a) compression and (b) tensile strain-stress cycles of as prepared hydrogels DCH4/20/2, which reflected the recoverability of the toughening mechanism. (c) Schematic illustration of the dissociation and reassociation of $\beta-\mathrm{CD} / \mathrm{ADA}$ complexes on the $\mathrm{CNC}$ surface. The (d) compression and (e) tensile strain-stress cycles of fully swollen hydrogels DCH4/20/2-S. (f) Schematic illustration of the dissociation of $\beta-\mathrm{CD} / \mathrm{ADA}$ complexes in the fully swollen hydrogels, where the sacrificed $\beta-\mathrm{CD} / \mathrm{ADA}$ complexes could not recover anymore.

In contrast, fully swollen hydrogels DCH4/20/2-S showed even narrower hysteresis loop in the second non-interval compression (Figure 4d), which revealed a more elastic mechanical behavior after stretching. In addition, unlike the as prepared hydrogels, the DCH4/20/2-S lost its recoverability of the energy dissipation system. Even after a $48 \mathrm{~h}$ static rest, the compressed samples were still not able to rebuild the energy dissipation system. During the tensile test of DCH4/20/2-S, the tensile strain-stress curve could not recover either (Figure 4e). Furthermore, because of the large deformation during the tensile tests, we also noticed a permanent elongation of the stretched hydrogels DCH4/20/2-S (Figure 4e). This elongation, together with the energy dissipation system, was not able to recover even after a long-time interval.

The obvious difference between the as prepared and fully swollen hydrogels could be explained 
by the highly heterogeneous hydrogel network and the presence of numerous autonomous dynamic conjunctions on the nanocrystals-polymer interface. Because of the in situ gelation approach, the dynamic nano-crosslinkers were well dispersed in the uniform polymer matrix. Therefore, the $\beta-\mathrm{CD} / \mathrm{ADA}$ complexes in the as prepared hydrogels did not undertake much stress and maintained reversible (Figure 4c).

However, the volume of hydrogels expanded and the distance between nano-crosslinkers increased during the swelling, so the polymer chains between nano-crosslinkers were stretched (Figure 4f). As mentioned earlier, the uneven swelling capability of the components in the composite hydrogels amplified the heterogeneity of hydrogels. The inhomogeneous swelling subsequently generated the stress concentration on the nano-crosslinker/polymer interface. In our hydrogels, the $\beta-\mathrm{CD} / \mathrm{ADA}$ complexes undertook the stress concentrated on the interface. This residual stress in fully swollen hydrogels would push $\beta-\mathrm{CD} / \mathrm{ADA}$ conjunctions away from equilibrium conditions and intend to dissociate. If there was no static scaffold to limit the volume expansion, the hydrogel network could be destroyed solely by swelling, as shown by hydrogels DCH4/20/1. In hydrogels DCH4/20/2, the hybrid crosslinking network maintained the physical integrity of hydrogels. Yet the heterogeneous swelling and stress concentration on the nano-crosslinker/polymer interface still significantly affected mechanical behaviors of the hydrogels. As shown in Figure 4f, when extra loading was applied to swollen hydrogels, the $\beta$ $\mathrm{CD} / \mathrm{ADA}$ complexes would easily dissociate and dissipate the elastic energy stored in the polymer chains. Therefore the swollen hydrogels were still toughened by $\beta-\mathrm{CD} / \mathrm{ADA}$ complexes. Nevertheless, once the extra loading was removed, the stretched polymer chains could form a network which was partially independent from $\mathrm{CNC}$. Once the $\beta-\mathrm{CD} / \mathrm{ADA}$ conjunctions were consumed in the fully swollen hydrogels, this sacrificed bonding could not be regenerated. A interesting contast can be found in the work from McKee et. al. ${ }^{[22]}$ With a three component host-guest supramolecular recognition, their hydrogels showed a unaffected self-healing capability. The most important reason would be, the $\beta-\mathrm{CD} / \mathrm{ADA}$ complexes in our system were directly settled on the CNC surface, rather than on the polymer bush surround CNC. This irreversible process could also explain the irreversible expansion of the stretched composite hydrogels (Figure 4d). Subsequently, the re-distribution of the nanocrosslinkers and 
polymer chains within the stretched networks led to irreversible change of hydrogel shape.

The stretchability of hydrogels was measured on both DCH4/20/2 and DCH4/20/2-S (Figure S15). DCH4/20/2 could be stretched to the 6.4 times of its original length. In the meantime, even softer, DCH4/20/2-S did not break until $\lambda$ reached 8.4. In addition, no matter as prepared or fully swollen hydrogels, the strain-stress curves both showed a yield point. The DCH4/20/2 showed an obvious yield point at $\lambda \sim 0.5$. In comparison, the yield process for DCH4/20/2-S was prolonged and terminated at $\lambda \sim 3.1$.

To compare with DCH4/20/2-S, the hydrogels "4/20/2(ADA blocked)-S” (ADA on CNC-ADA was blocked by $\beta-\mathrm{CD}$ before the in situ polymerization with $\beta-\mathrm{CD}-\mathrm{Ac}_{2}$ and $\mathrm{AAm}$ ) were prepared. They showed a smaller elastic modulus and smaller elongation ratio. Another type of hydrogels consisting of polymerized $\beta-\mathrm{CD}-\mathrm{Ac}_{2}$ and AAm without CNC-ADA after fully swelling ( $\left.\beta-\mathrm{CD}-\mathrm{Ac}_{2} / \mathrm{PAAm}-\mathrm{S}\right)$ showed even smaller elastic modulus and lower elongation ratio than hydrogels DCH4/20/2-S (Figure 5a). Figure 5b showed the real state of hydrogel DCH4/20/2-S that was stretched to 3 times and 8 times of its original length. The pores in the stretched hydrogels were largely orientated pores along the deformation in the macro-view (Figure 5b). In addition, the highly fibrous microstructure in the stretched hydrogels also reflected the relocation of hydrogel crosslinking network. The covalent crosslinking network partially left CNC surface, which were able to further expand, as illustrated in Figure 4f. 

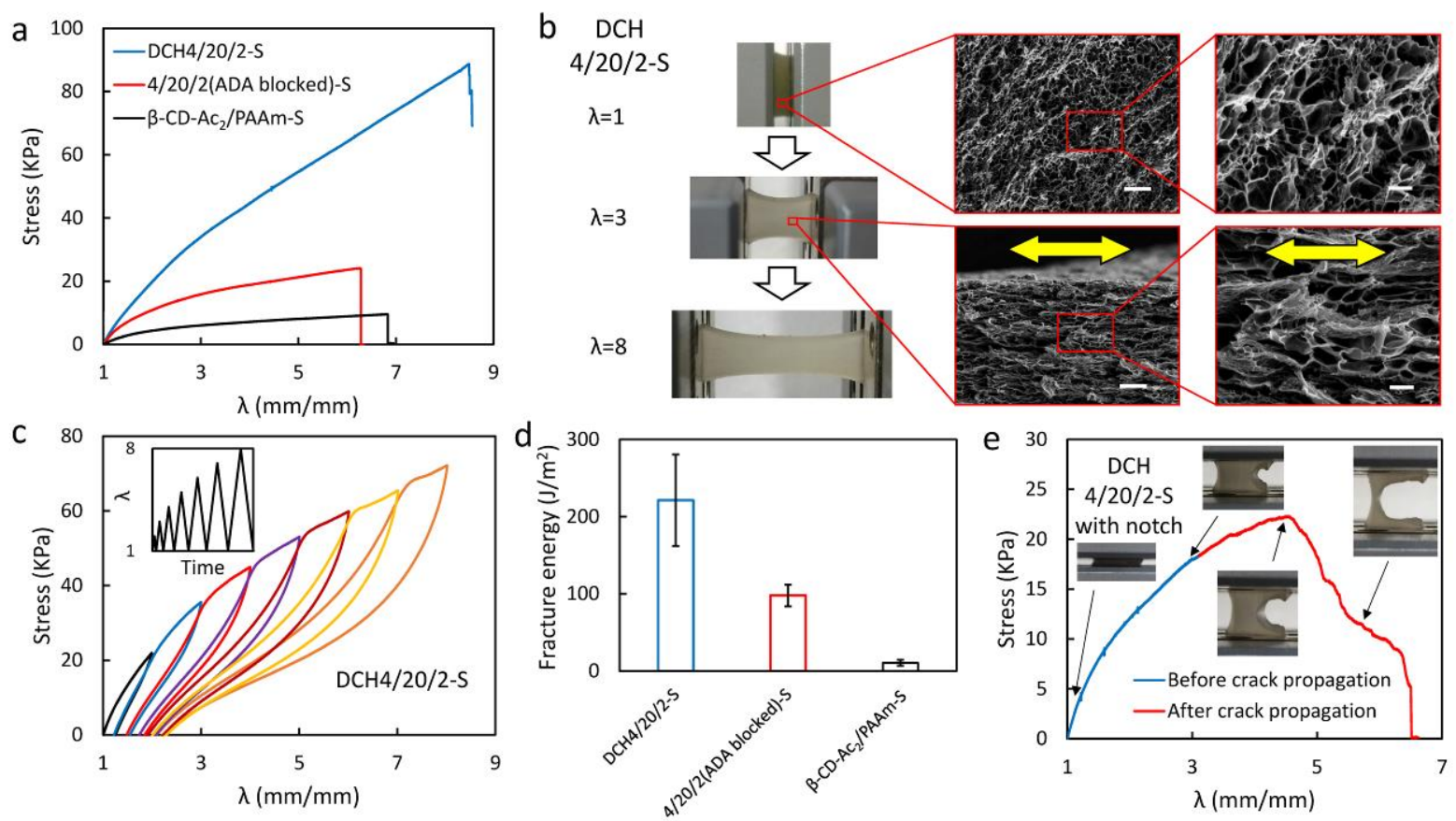

Figure 5. Mechanical properties of fully swollen dynamic composite hydrogels. (a) The tensile tests of fully swollen hydrogel DCH4/20/2-S and its control groups. (b) Photographs of the stretched DCH4/20/2-S with $\lambda=1,3$ and 8; as well as the SEM images of original and stretched $(\lambda=3)$ hydrogels with scale bars in the left SEM images of $10 \mu \mathrm{m}$ and $2 \mu \mathrm{m}$ in the right SEM images. The hydrogel specimens were fast immersed in liquid nitrogen to fix their microstructure, and water was removed by freeze-drying. (c) The step-up tensile strain-stress curves of DCH4/20/2-S, showing that the consumption of energy dissipation system was correlated to the deformation. (d) Hydrogel fracture energy of hydrogels DCH4/20/2-S and the control groups. (e) The tensile strain-stress curves of the hydrogels DCH4/20/2-S with preset notch showed when the crack started to propagate and how the crack went through the whole hydrogel.

The fully swollen hydrogels clearly showed a "Mullins effect" (Figure 5c), which is a common phenomenon for carbon filled elastomers and hydrogels toughened by sacrificed bonding. ${ }^{[6]}$ The previous investigation from Yang et. al. provided a clear contrast. With methacrylatemodified $\mathrm{CNC},{ }^{[20]}$ the covalently crosslinked CNC-polyacrylamide hydrogels showed very narrow hysteresis area during the loading and unloading tensile tests, which indicated its high elasticity and full recoverability. The step-up tensile tests of hydrogels DCH4/20/2-S indicated 
a clear deformation dependency of sacrificed mechanism. In the as prepared DCH4/20/2, the "Mullins effect" was less obvious, especially at small deformation $(\lambda<3)$. The reason should be the partial recoverability of sacrificed $\beta-\mathrm{CD} / \mathrm{ADA}$ complexes in the as prepared hydrogels during the tensile tests. In addition, the unfolding of loose polymer chains in the as prepared hydrogels also acted as another energy dissipation system (Figure S16).

The fracture toughness of dynamic composite hydrogels was analyzed as well. We used the method reported by Sun et al. ${ }^{[4]}$ The hydrogel strips with a preset notch $(30 \%$ of specimen width) were stretched and the "critical elongation" was recorded until the crack started to propagate. The fracture energy was calculated by the area under strain-stress curve of the hydrogels (without notch) until the "critical elongation" obtained from notched specimens. The hydrogel DCH4/20/2-S showed a larger fracture energy than hydrogel 4/20/2(ADA blocked)$\mathrm{S}$ and $\beta-\mathrm{CD}-\mathrm{Ac}_{2} / \mathrm{PAAm}-\mathrm{S}$, which was due to the existence of $\beta-\mathrm{CD} / \mathrm{ADA}$ toughening mechanism (Figure 5d).

In addition to investigate when the crack started to propagate, the process of crack propagation was further analyzed. The propagation of crack in our dynamic composite hydrogels was not a continuous process. According to the strain-stress curve and the snapshots in Figure 5e (prenotched hydrogel DCH4/20/2-S), the crack started to propagate at $\lambda \sim 3.1$ and the crack spent several minutes to go through the whole specimen. During this process, the propagation of the crack paused and restarted with certain intervals until the complete breakup. As the comparison, the crack propagation in $4 / 20 / 2$ (ADA blocked)-S and $\beta-\mathrm{CD}-\mathrm{Ac}_{2} / \mathrm{PAAm}-\mathrm{S}$ was much faster, normally within a few seconds and also consumed much less energy (Figure S17). Furthermore, the crack area of DCH4/20/2-S was characterized using SEM (Figure S18). As shown in Figure. S18b, the surface generated by crack propagation had a fluffy morphology, which indicated the local plastic deformation during the crack development. At the end of the crack propagation (Figure S18c), even more fibrous structure was observed and the pores were oriented along the crack opening. In the dynamic composite hydrogels, because of the heterogeneous structure and dynamic nanocrystals-polymer interface, the crack more likely to propagate along the loadsensitive interface around $\mathrm{CNC}$. On this interface, the gradual dissociation of the $\beta-\mathrm{CD} / \mathrm{ADA}$ 
conjunctions continuously resisted the crack development. Furthermore, the crack growth along the nano-crosslinkers could also lead to a circuitous pathway, which could also retard the crack propagation.

\section{Conclusion}

In conclusion, we prepared a novel kind of dynamic composite hydrogels with a heterogeneous structure based on the pre-organized dynamic CNC nano-crosslinkers. The crosslinking agents, variously acrylated $\beta-\mathrm{CD}$, were immobilized on the $\mathrm{CNC}$ surface via pre-organized host-guest interaction between $\beta-\mathrm{CD}$ and $\mathrm{ADA}$. These variously acrylated $\beta-\mathrm{CD}$ contributed to further construction of hydrogels via purely physical or hybrid chemical/physical interactions. Yet, the preset $\beta-\mathrm{CD} / \mathrm{ADA}$ conjunctions between polymer chains and $\mathrm{CNC}$ are always the physical interaction. Because of the highly heterogeneous structure, the conjunctions on the CNCpolymer interface exhibited gradual detachment under exerted stress and worked as an effective energy dissipation system. The sacrificed $\beta-\mathrm{CD} / \mathrm{ADA}$ conjunction showed a time dependent recoverability in the as prepared hydrogels. However, this recoverability was lost when the hydrogels got fully swollen, even they were still toughened by the same energy dissipation system. This phenomenon was due to the highly heterogeneous structure caused by swelling. Along with the volume increase, the uneven expansion of nano-crosslinkers and flexible polymers generated residual stress on the $\mathrm{CNC}$-polymer interface, and therefore resisted the recovery of the sacrificed $\beta-\mathrm{CD} / \mathrm{ADA}$ conjunction.

In addition to improving mechanical properties, the dynamic nano-crosslinkers within the hydrogels also significantly delayed the propagation of the crack. The crack propagation during the stretching of pre-notched hydrogels was paused and restarted until the complete breakup. This could be attributed to the loading sensitive bonding on the CNC surface. The sequential dissociation of the $\beta-\mathrm{CD} / \mathrm{ADA}$ complexes and the heterogeneous hydrogel structures effectively retarded the crack propagation.

Our heterogeneous dynamic hydrogels contained a toughening mechanism located on the nanocrosslinker surface. In addition to the improvement of mechanical properties, this 
heterogeneous structure also led to the redistribution of crosslinking network under stress and gathered the feasibility to retard the crack development. Based on these, we provided a special strategy to design tough and functional hydrogels with heterogeneous structures that will be useful for a wide range of purposes.

\section{Acknowledgements}

The program 'Material Science Wood' from Lower Saxony Ministry of Science and Culture, Germany, Fonds der Chemischen Industrie (FCI), and the start-up funding for the Promotion of Young Academics of Georg-August-University of Göttingen are gratefully acknowledged for the financial support. The authors also thank Christina Jayachandran, Michael Hendrich, Professor Philipp Vana and Professor Carsten Mai for the experimental assistance and valuable discussion.

\section{References}

[1] J. Thiele, Y. Ma, S. M. Bruekers, S. Ma, W. T. Huck, Adv. Mater. 2014, 26, 125; E. A. Appel, J. del Barrio, X. J. Loh, O. A. Scherman, Chem. Soc. Rev. 2012, 41, 6195; F. Rehfeldt, A. J. Engler, A. Eckhardt, F. Ahmed, D. E. Discher, Adv. Drug Deliv. Rev. 2007, 59, 1329

[2] X. Zhao, Soft Matter 2014, 10, 672.

[3] J. P. Gong, Y. Katsuyama, T. Kurokawa, Y. Osada, Adv. Mater. 2003, 15, 1155; Q. Chen, L. Zhu, C. Zhao, Q. Wang, J. Zheng, Adv. Mater. 2013, 25, 4171; H. J. Zhang, T. L. Sun, A. K. Zhang, Y. Ikura, T. Nakajima, T. Nonoyama, T. Kurokawa, O. Ito, H. Ishitobi, J. P. Gong, Adv. Mater. 2016, 28, 4884; V. X. Truong, M. P. Ablett, S. M. Richardson, J. A. Hoyland, A. P. Dove, J. Am. Chem. Soc. 2015, 137, 1618.

[4] J. Y. Sun, X. Zhao, W. R. Illeperuma, O. Chaudhuri, K. H. Oh, D. J. Mooney, J. J. Vlassak, Z. Suo, Nature 2012, 489, 133.

[5] J. P. Gong, Soft Matter 2010, 6, 2583.

[6] R. E. Webber, C. Certon, H. R. Brown, J. P. Gong, Macromolecules 2007, 40, 2919.

[7] F. Luo, T. L. Sun, T. Nakajima, T. Kurokawa, Y. Zhao, K. Sato, A. B. Ihsan, X. Li, H. Guo, J. P. Gong, Adv. Mater. 2015, 27, 2722; A. Bin Imran, K. Esaki, H. Gotoh, T. Seki, K. Ito, Y. Sakai, Y. Takeoka, Nat. Commun. 2014, 5, 5124. 
[8] S. Hou, P. X. Ma, Chem. Mater. 2015, 27, 7627; S. C. Grindy, R. Learsch, D. Mozhdehi,

J. Cheng, D. G. Barrett, Z. Guan, P. B. Messersmith, N. Holten-Andersen, Nat. Mater. 2015, $14,1210$.

[9] M. Guo, L. M. Pitet, H. M. Wyss, M. Vos, P. Y. Dankers, E. W. Meijer, J. Am. Chem. Soc. 2014, 136, 6969.

[10]M. Nakahata, Y. Takashima, A. Harada, Macromol. Rapid Commun. 2015, 37, 86; M. Tan, Y. Cui, A. Zhu, H. Han, M. Guo, M. Jiang, Polym. Chem. 2015, 6, 7543; K. Wei, M. Zhu, Y. Sun, J. Xu, Q. Feng, S. Lin, T. Wu, J. Xu, F. Tian, J. Xia, G. Li, L. Bian, Macromolecules 2016, 49,866 .

[11] S. Lv, D. M. Dudek, Y. Cao, M. M. Balamurali, J. Gosline, H. Li, Nature 2010, 465, 69; J. Fang, A. Mehlich, N. Koga, J. Huang, R. Koga, X. Gao, C. Hu, C. Jin, M. Rief, J. Kast, D. Baker, H. Li, Nat. Commun. 2013, 4, 2974.

[12]Q. Wang, Z. Gao, J. Mech. Phys. Solids 2016, 94, 127.

[13]P. Thoniyot, M. J. Tan, A. A. Karim, D. J. Young, X. J. Loh, Adv. Sci. 2015, 2, 1400010.

[14]A. Klein, P. G. Whitten, K. Resch, G. Pinter, J. Polym. Sci., Part B: Polym. Phys. 2015, 53, 1763; L. W. Xia, R. Xie, X. J. Ju, W. Wang, Q. Chen, L. Y. Chu, Nat. Commun. 2013, 4, 2226.

[15] K. Haraguchi, T. Takehisa, Adv. Mater. 2002, 14, 1120; Z. Hu, G. Chen, Adv. Mater. 2014, 26, 5950; Q. Wang, J. L. Mynar, M. Yoshida, E. Lee, M. Lee, K. Okuro, K. Kinbara, T. Aida, Nature 2010, 463, 339.

[16]E. A. Appel, M. W. Tibbitt, M. J. Webber, B. A. Mattix, O. Veiseh, R. Langer, Nat. Commun. 2015, 6, 6295.

[17]Q. Li, D. G. Barrett, P. B. Messersmith, N. Holten-Andersen, ACS nano 2016, 10, 1317.

[18]Y. Habibi, Chem. Soc. Rev. 2014, 43, 1519.

[19]J. Yang, C. Han, J. Duan, F. Xu, R. Sun, ACS Appl. Mater. Interfaces 2013, 5, 3199; J. Yang, M. Ma, X. Zhang, F. Xu, Macromolecules 2016, 49, 4340.

[20] J. Yang, C.-R. Han, J.-F. Duan, M.-G. Ma, X.-M. Zhang, F. Xu, R.-C. Sun, Cellulose 2012, $20,227$. 
[21]J. R. McKee, S. Hietala, J. Seitsonen, J. Laine, E. Kontturi, O. Ikkala, ACS Macro Lett. 2014, 3, 266.

[22]J. R. McKee, E. A. Appel, J. Seitsonen, E. Kontturi, O. A. Scherman, O. Ikkala, Adv. Funct. Mater. 2014, 24, 2706.

[23]H. Kamata, Y. Akagi, Y. Kayasuga-Kariya, U. I. Chung, T. Sakai, Science 2014, 343, 873. 


\section{Publication 1 (Supporting information)}

\section{Robust heterogeneous hydrogels with dynamic nanocrystals-polymer interface}

Heqin Huang, Yonggui Wang, Xiaojie Wang, Florian Rehfeldt and Kai Zhang*

\section{Materials}

Microcrystalline cellulose (MCC), 2,2,6,6-tetramethylpiperidine 1-oxyl (TEMPO), cysteamine, p-toluenesulfonyl chloride ( $\mathrm{Tos} C l$ ), sodium chlorite, sodium azide $\left(\mathrm{NaN}_{3}\right)$ and phosphotungstic acid hydrate were purchased from Sigma-Aldrich (USA). Adamantanol (ADA-OH), acryloyl chloride and $\beta$-cyclodextrin ( $\beta-\mathrm{CD}$ ) were obtained from Alfa Aesar (USA). Triethylamine, ammonium persulfate (APS), N,N,N',N'-Tetramethylethylenediamine (TEMED) and acrylamide (AAm) were bought from Merck Millipore (USA). N-acryloxysuccinimide was received from Acros Organics (USA). Carbonyldiimidazole and sodium hydroxide were ordered from VWR (Germany). Azobisisobutyronitrile (AIBN) was bought from TCI (Japan). Triphenylphosphine was obtained from Fluorochem (USA). The organic solution, including dichloromethane (DCM), N,N-dimethylformamide (DMF) and acetone were all purchased from TH. Geyer (Germany). DI water utilized throughout all experiments was purified from a Millipore system.

\section{Experimental section}

\section{The preparation of $C N C-A D A$}

CNC were prepared with TEMPO-mediated oxidation of MCC according to previous report. ${ }^{[1]}$ To remove large particles within the $\mathrm{CNC}$, the $\mathrm{CNC}$ water suspension (about $6 \mathrm{mg} / \mathrm{ml}$ ) was put in the fridge at $4^{\circ} \mathrm{C}$ for overnight. Then, the upper stable suspension was used for following experiments. The ratio of the surface-exposed carboxylic to carbonyl groups of $\mathrm{CNC}$ was determined via electric conductivity titration. The content of carboxylic groups was evaluated 
using $10 \mathrm{ml}$ of aqueous $\mathrm{CNC}$ suspension where the $\mathrm{pH}$ value was adjusted to 2 with $0.1 \mathrm{M} \mathrm{HCl}$. The suspension solution was titrated with $0.1 \mathrm{M} \mathrm{NaOH}$ using the 800 Dosimat (Metrohm) employing a dosing rate of $0.01 \mathrm{ml} / \mathrm{s}$, while the conductivity was recorded using the 856 Conductivity Module (Metrohm) with an interval of $2 \mathrm{~s}$. In order to determine the content of aldehyde groups, the $\mathrm{CNC}$ suspension was oxidized with $\mathrm{NaClO}_{2}$ at ambient temperature and $\mathrm{pH}$ 4-5. Then, the total carboxylic groups were titrated again. The difference between preoxidized and oxidized CNC was calculated, which is equal to the content of aldehyde groups.

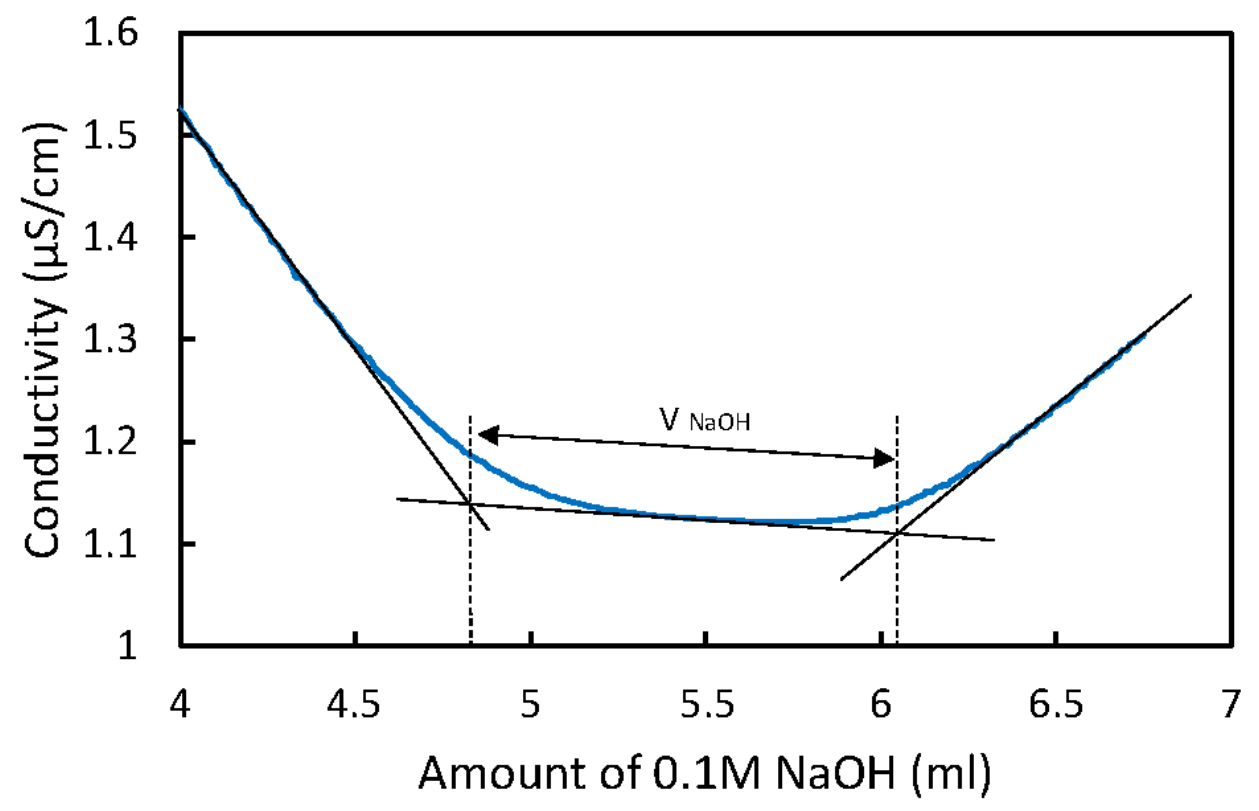

Figure S1. Conductive titration curve of CNC.

The surface of virgin CNC was decorated with carboxyl groups of about $1.52 \mathrm{mmol} / \mathrm{g}$. Then CNC were repeatedly washed with acetone and DMF, then $300 \mathrm{mg}$ CNC was redispersed in 15 mL DMF. Carboxyl groups on the CNC surface were activated by 1,1'-carbonyldiimidazole (70 mg, $0.43 \mathrm{mmol}$ ) for $30 \mathrm{~min}$. Then, cysteamine (174 mg, $2.3 \mathrm{mmol}$ ) was mixed with CNC suspension. The amidation reaction was performed at $80^{\circ} \mathrm{C}$ under $\mathrm{N}_{2}$ protection for $48 \mathrm{~h}$. The resulted CNC-SH was then washed with DMF for three times. Meanwhile, Acrylate adamantine (ADA-Ac) were prepared by adaptation of similar reported method. ${ }^{[2]}$ Adamantanol $(2.0 \mathrm{~g}, 13.06 \mathrm{mmol})$ and triethylamine $(3.70 \mathrm{~mL}, 26.5 \mathrm{mmol})$ were dissolved in 
$20 \mathrm{~mL}$ dry dichloromethane. The resulted mixture was cooled to $5^{\circ} \mathrm{C}$, and then acryloyl chloride $(2.12 \mathrm{~mL}, 26.08 \mathrm{mmol})$ dissolved in $15 \mathrm{~mL}$ dichloromethane was added dropwise within $30 \mathrm{~min}$. The mixture was continuously stirred at $4^{\circ} \mathrm{C}$ for $48 \mathrm{~h}$. The obtained mixture was firstly filtered and then trimethylamine $\mathrm{HCl}$ salts were removed by repeated washing with saturated sodium bicarbonate, water and saturated saline solution. The dichloromethane layer was dried with sodium sulfate anhydrous. Eventually, dichloromethane was removed under reduced pressure, leading to the final product ( $1.9 \mathrm{~g}, 9.21 \mathrm{mmol}$, and yield $70.5 \%$ ).

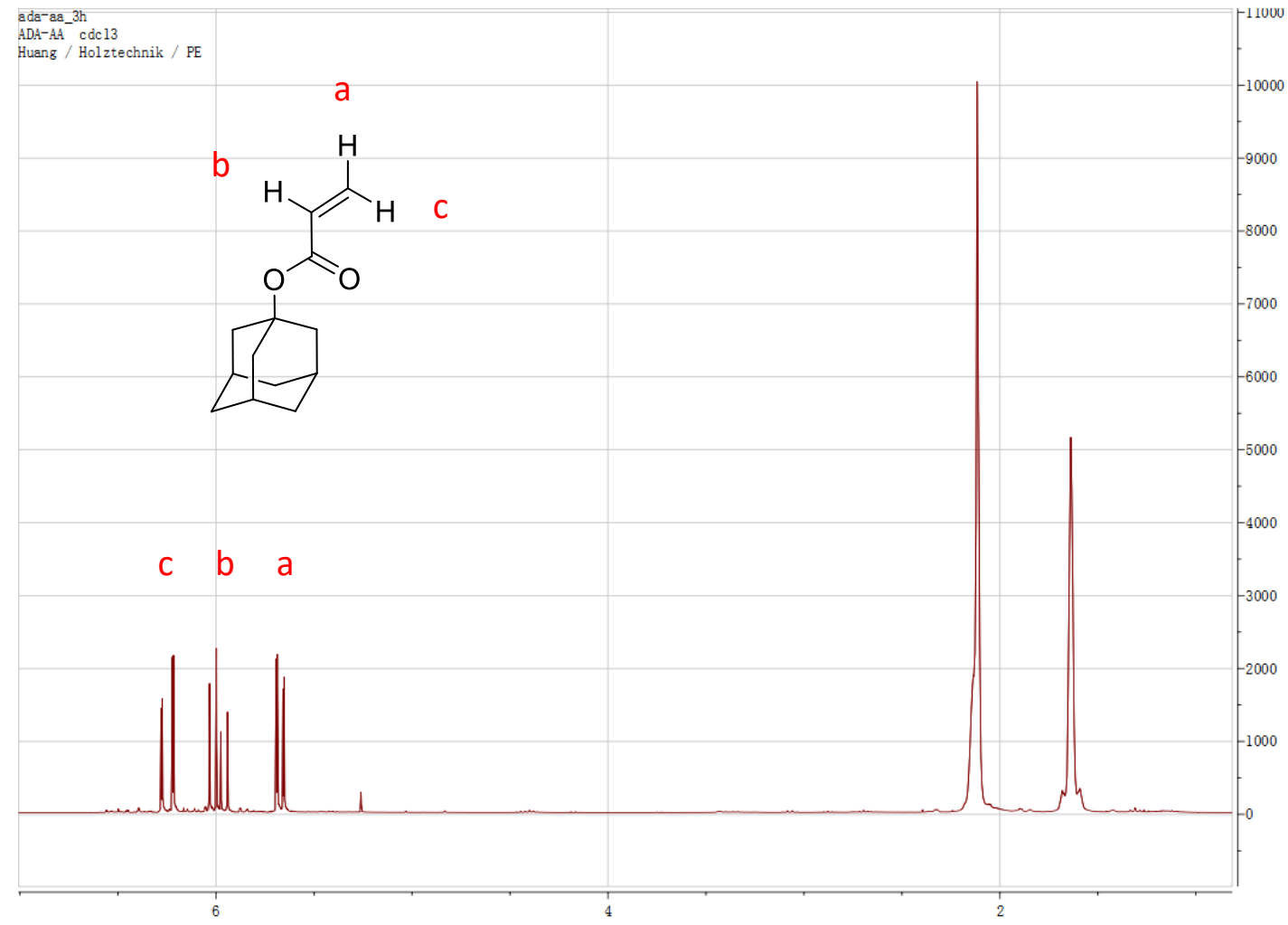

Figure S2. NMR spectrum of ADA acrylate (in chloroform-d).

The obtained ADA-Ac was immobilized on the surface of CNC-SH via thiol-ene click reaction. $150 \mathrm{mg}$ CNC-SH was redispersed in $15 \mathrm{ml}$ dry DMF, and ADA-Ac (225 mg, $1.1 \mathrm{mmol})$ and azobisisobutyronitrile (AIBN) $(20 \mathrm{mg}, 0.12 \mathrm{mmol})$ were added, the immobilization was achieved at $65^{\circ} \mathrm{C}$ for $24 \mathrm{~h}$. The CNC-ADA was washed gradually to acetone and eventually to water. 


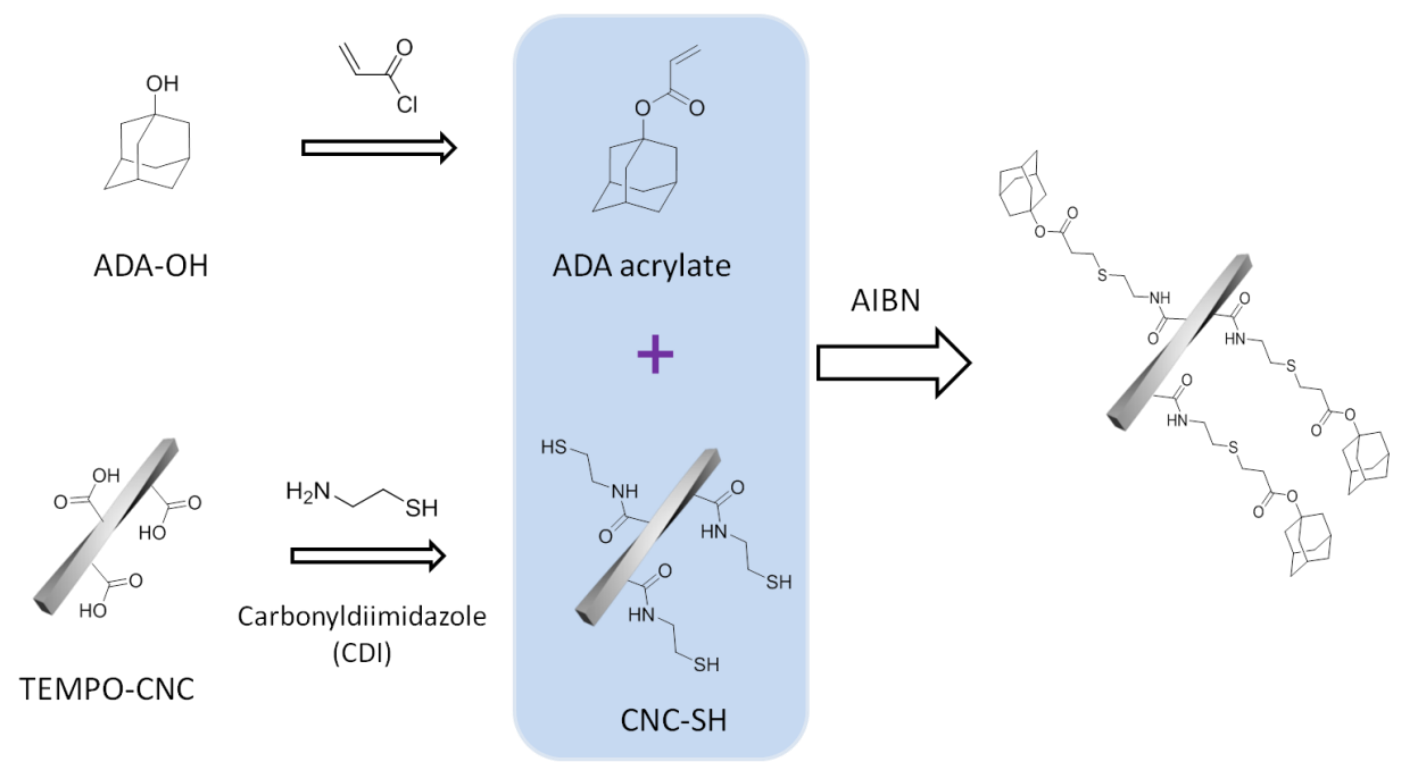

Figure S3. Schematic representation for the synthesis of CNC-ADA.

According to the results of elemental analysis. The content of thiol groups on CNC-SH was calculated as:

$\mathrm{N}=14 * \mathrm{x}$

(1)

Where $\mathrm{N}$ was the nitrogen weight content of CNC-SH; 14 was the molecular weight of nitrogen $(\mathrm{g} / \mathrm{mol})$; $\mathrm{x}$ indicated the molar content of cysteamine on $\mathrm{CNC}-\mathrm{SH}(\mathrm{mol} / \mathrm{g})$, which was the density of thiol groups on CNC-SH.

The content of ADA on CNC-ADA was calculated as:

$\mathrm{N} /(1+206.3 * \mathrm{y})=\mathrm{N}^{\prime}$

(2)

Where $\mathrm{N}$ was the nitrogen weight content in $\mathrm{CNC}-\mathrm{SH}$; N' was the nitrogen weight content in CNC-ADA; 206.3 was the molecular weight of ADA-Ac (g/mol); y indicated the molar density of ADA-Ac grafted on CNC-SH (mol/g).

The amount of thiol groups on the CNC-SH surface was determined to be $0.91 \mathrm{mmol} / \mathrm{g}$. The content of ADA on CNC-ADA was calculated to be $0.67 \mathrm{mmol} / \mathrm{g}$. In another word, $0.96 \mathrm{mmol} / \mathrm{g}$ 
carboxyl groups on original CNC were modified with ADA in CNC-ADA.

AFM

AFM imaging of CNC-ADA was performed on an MFP-3D system (Asylum Research, Santa Barbara, USA). Measurements were done in AC mode using the shorter lever of an Olympus AC-160 cantilever (Asylum Research, Santa Barbara, USA) at a driving frequency of $330 \mathrm{kHz}$. The lever has a sharpened tetrahedral tip with a typical tip radius of $7 \mathrm{~nm}$ and a nominal spring constant of $26 \mathrm{~N} / \mathrm{m}$. Scans of the size $2.1 \times 2.1 \mu \mathrm{m}^{2}$ were recorded with a scan rate of $1 \mathrm{~Hz}$ and 256 by 256 points by lines.
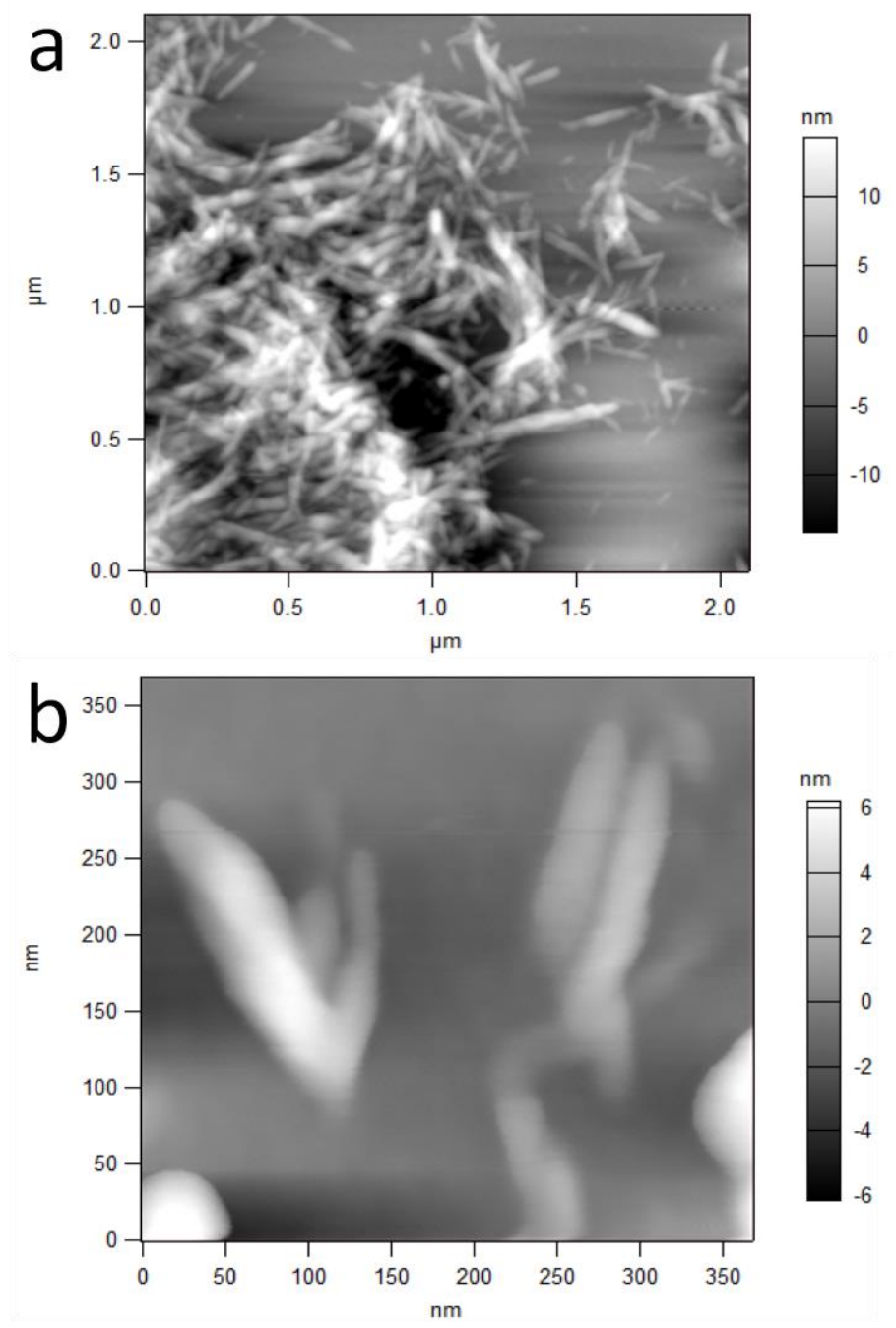

Figure S4. AFM images of CNC-ADA at two different magnifications. 


\section{TEM}

Dried CNC-ADA was also characterized with TEM, and the CNC-ADA was prepared from its suspension in water of $0.01 \mathrm{wt} \%$. The specimen was stained by phosphotungstic acid solution $(0.2 \mathrm{wt} \%$ in water, $\mathrm{pH}$ was adjusted to 7.0 by $1 \mathrm{M} \mathrm{NaOH})$. The TEM observation was performed on a CM 12 Transmission Electron Microscope (Philips, Netherland).

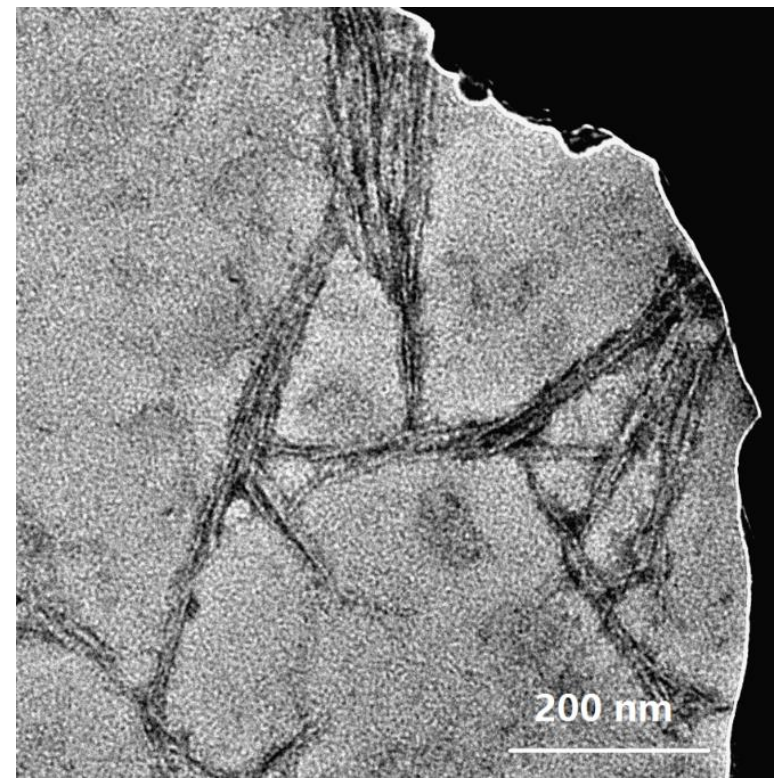

Figure S5. TEM images of CNC-ADA.
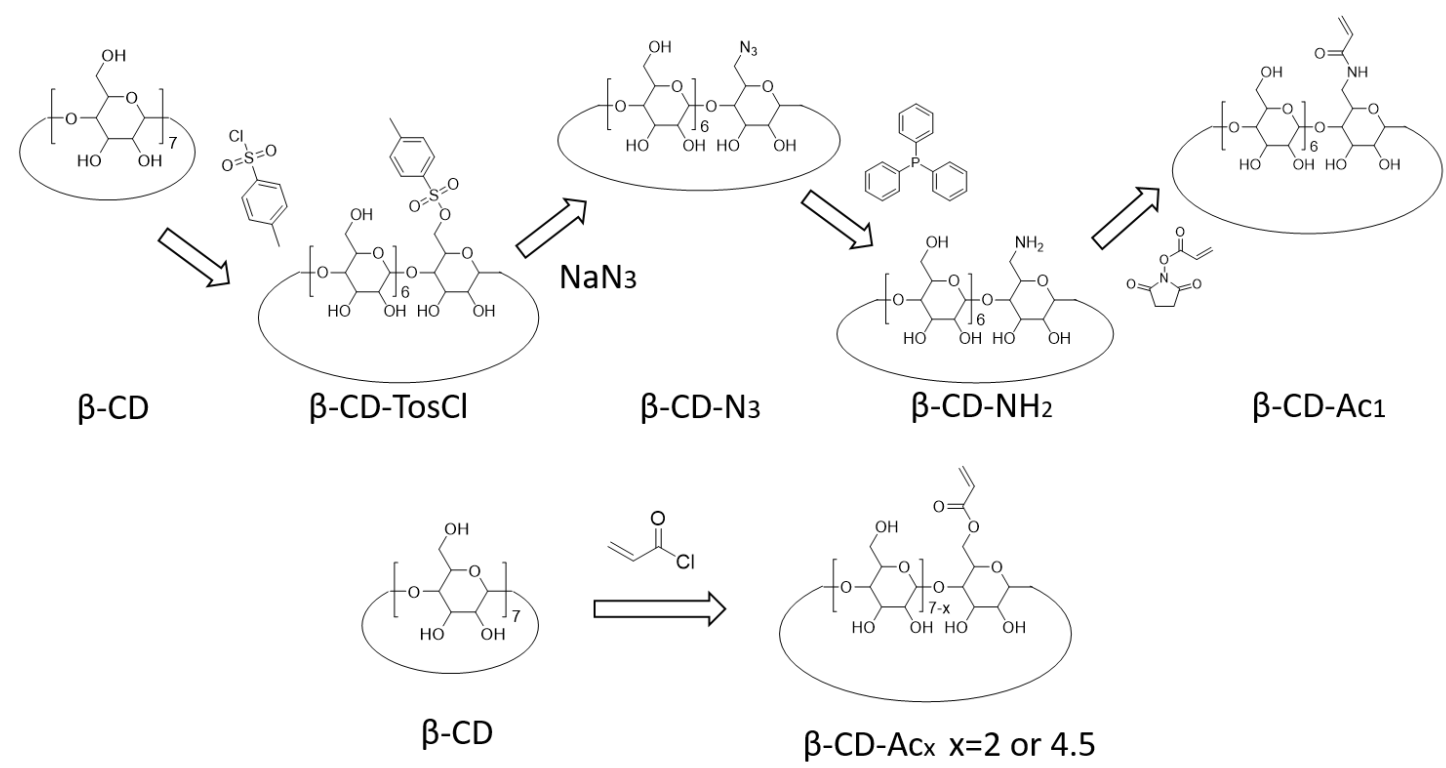

Figure S6. Schematic representation for the synthesis of $\beta-C D-A c_{x}$. 


\section{The preparation of $\beta-C D-A c_{1}$}

The synthesis of 6-deoxy-6-amino- $\beta$-cyclodextrin $\left(\beta-\mathrm{CD}-\mathrm{NH}_{2}\right)$ was performed according to a reported method with modifications. ${ }^{[3]} \beta-\mathrm{CD}(10 \mathrm{~g}, 8.81 \mathrm{mmol})$ was suspended in $0.75 \mathrm{M}$ $\mathrm{NaOH}(200 \mathrm{~mL})$ and cooled to $5^{\circ} \mathrm{C}$. p-Toluenesulfonyl chloride (TosCl; $\left.3.36 \mathrm{~g}, 17.6 \mathrm{mmol}\right)$ was dissolved in $10 \mathrm{~mL}$ acetonitrile and added dropwise within $30 \mathrm{~min}$. The reaction was stirred at $5^{\circ} \mathrm{C}$ for $1 \mathrm{~h}$ and another $2 \mathrm{~h}$ at room temperature. The precipitate was removed by filtration and the filtrate was neutralized with $10 \mathrm{wt} \%$ and $1 \mathrm{M} \mathrm{HCl}$. The crude product was recrystallized from cold water $(3 \times 75 \mathrm{~mL})$, and dried at $60^{\circ} \mathrm{C}$ to afford the intermediate 6-o-monotosyl-6deoxy- $\beta$-cyclodextrin ( $\beta$-CD-TosCl, $3.05 \mathrm{~g}, 2.37 \mathrm{mmol}$, yield 26.9\%) as a white powder. A round-bottom flask was charged with $\beta$-CD-TosCl (3 g, 2.33 mmol), $\mathrm{NaN}_{3}$ (1.91 g, $29.38 \mathrm{mmol}$ ) and $\mathrm{H}_{2} \mathrm{O}(50 \mathrm{~mL})$. The reaction was refluxed for $4 \mathrm{~h}$. The product was precipitated from cold acetone $(3 \times 300 \mathrm{~mL})$ and dried at $60^{\circ} \mathrm{C}$ to afford the 6 -deoxy-6-azide- $\beta$-cyclodextrin $\left(\beta-\mathrm{CD}-\mathrm{N}_{3}\right)$ as a white powder. $2.8 \mathrm{~g} \beta-\mathrm{CD}-\mathrm{N}_{3}(1.42 \mathrm{mmol}), 1.4 \mathrm{~g}$ triphenylphosphine $(5.36 \mathrm{mmol})$ and 8 $\mathrm{mL}$ DMF were mixed in a $50 \mathrm{~mL}$ round bottle flask and degassed by $\mathrm{N}_{2}$ purge, until the mixture formed clear solution after about $2 \mathrm{~h}$ stirring. Then, $0.8 \mathrm{~mL} \mathrm{H}_{2} \mathrm{O}$ was added to the solution with syringe and the reaction was heated at $90^{\circ} \mathrm{C}$ for $3 \mathrm{~h}$. The product was washed with acetone for 3 times and obtained as mono-6-amino-deoxy-6-cyclodextrin ( $\left.\beta-\mathrm{CD}-\mathrm{NH}_{2}\right) . \beta-\mathrm{CD}-\mathrm{NH}_{2}(2.5 \mathrm{~g}$, $2.2 \mathrm{mmol})$ and $\mathrm{N}$-acryloxysuccinimide $(0.389 \mathrm{~g}, 2.3 \mathrm{mmol})$ was dissolved in $30 \mathrm{~mL}$ dimethyl sulfoxide (DMSO), and the reaction mixture was stirred overnight at room temperature after $\mathrm{N}_{2}$ purge for $15 \mathrm{~min}$. The resulted acryloyl-6-amino-6-deoxy- $\beta$-cyclodextrin $\left(\beta-\mathrm{CD}-\mathrm{Ac}_{1}\right)$ was repeatedly washed with cold acetone and dried at $60^{\circ} \mathrm{C}$. The acrylamide substitution ratio was calculated as 1.03 according to $1 \mathrm{H}$ NMR. Elemental analysis, calculation: C 45.49\%; H 6.19\%; N 1.18\%, found: C 45.19\%; H 6.34\%; N 1.26\%. Positive-ion matrix-assisted laser desorption/ionization time-of-flight mass spectrometry (MALDI-TOF-MS) analysis was performed on a Bruker autoflex speed mass spectrometer using 2,5-dihydroxy-benzoic acid as matrix. $\beta-\mathrm{CD}-\mathrm{NH}_{2}: \quad \mathrm{m} / \mathrm{z}=1157.4\left(\left[\mathrm{C}_{42} \mathrm{H}_{71} \mathrm{NO}_{34}+\mathrm{Na}\right]^{+}=1157.1\right) ; \beta-\mathrm{CD}-\mathrm{Ac}_{1}: \mathrm{m} / \mathrm{z}=1211.5$ $\left(\left[\mathrm{C}_{45} \mathrm{H}_{73} \mathrm{NO}_{35}+\mathrm{Na}\right]^{+}=1211.1\right)$. 


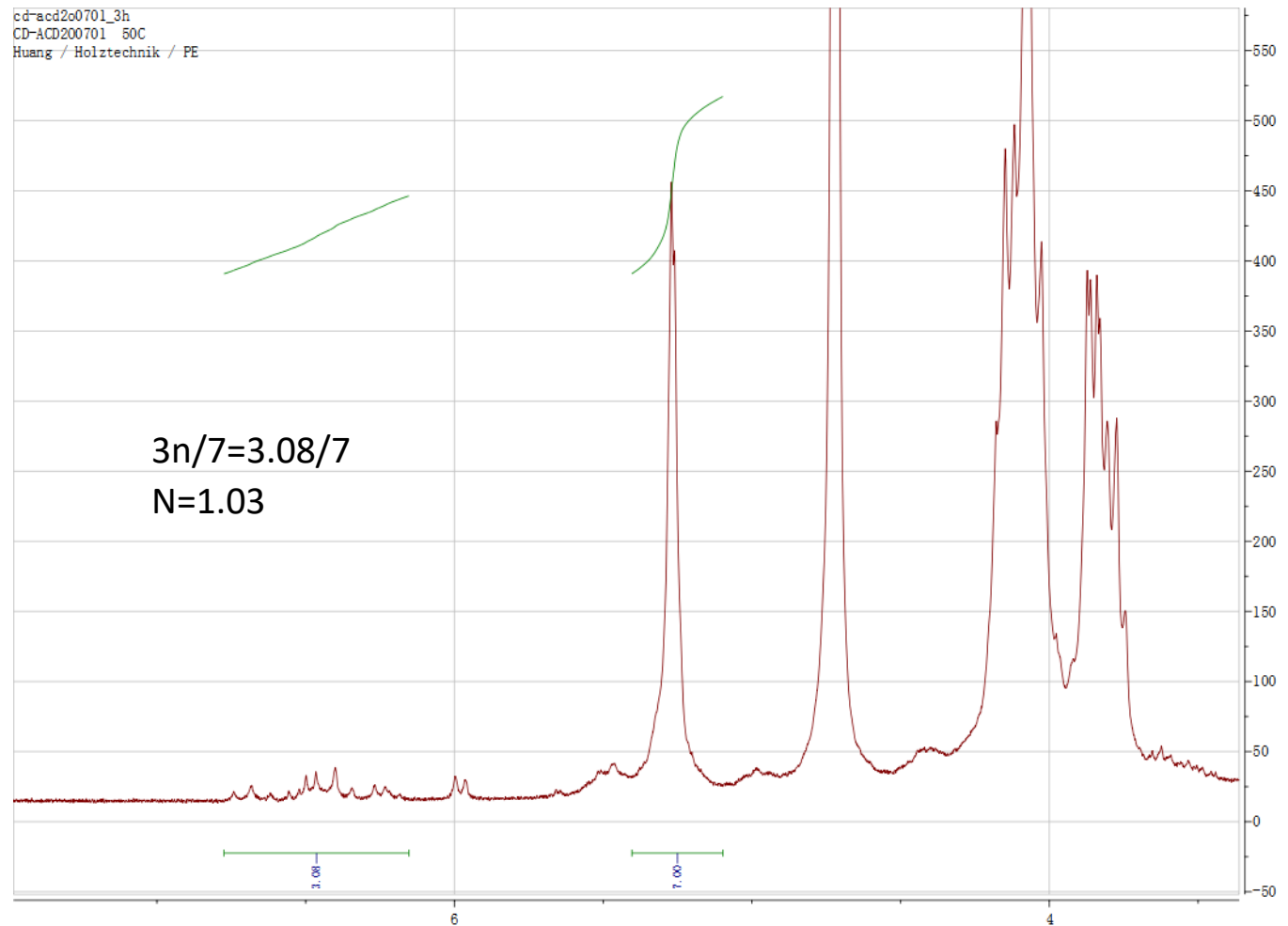

Figure S7. NMR spectrum of $\beta-\mathrm{CD}-\mathrm{Ac}_{1}\left(\right.$ in $\left.\mathrm{D}_{2} \mathrm{O}\right)$.

\section{The preparation of $\beta-C D-A c_{2}$}

$\beta-C D(2 \mathrm{~g}, 1.76 \mathrm{mmol})$ and triethylamine $(2.5 \mathrm{~mL}, 18.1 \mathrm{mmol})$ were dissolved in $20 \mathrm{~mL}$ DMF and purged by $\mathrm{N}_{2}$ gas for $20 \mathrm{~min}$. Then, the reaction mixture was cooled to below $5^{\circ} \mathrm{C} .1 .4 \mathrm{~mL}$ acryloyl chloride $(17.2 \mathrm{mmol})$ in $10 \mathrm{~mL}$ DMF was dropwisely added to the reaction. After $8 \mathrm{~h}$ stirring, the resulted mixture was filtrated, precipitated in acetone, and then washed with acetone for 3 times. The final product was obtained as white powder. The acrylate substitution ratio was calculated as 2.01 according to $1 \mathrm{H}$ NMR. Elemental analysis, calculation: $\mathrm{C} 46.38 \%$; H 6.00\%, found: C 46.04\%; H 6.13\%. MALDI-TOF-MS, $\beta-C D-A c_{1}: \mathrm{m} / \mathrm{z}=1211.8\left(\left[\mathrm{C}_{45} \mathrm{H}_{72} \mathrm{O}_{36}\right.\right.$ $\left.+\mathrm{Na}]^{+}=1212.0\right) ; \beta-\mathrm{CD}-\mathrm{Ac}_{2}: \mathrm{m} / \mathrm{z}=1265.8\left(\left[\mathrm{C}_{48} \mathrm{H}_{74} \mathrm{O}_{37}+\mathrm{Na}^{+}=1266.1\right) ; \beta-\mathrm{CD}-\mathrm{Ac}_{3}: \mathrm{m} / \mathrm{z}\right.$ $=1319.7\left(\left[\mathrm{C}_{51} \mathrm{H}_{76} \mathrm{O}_{38}+\mathrm{Na}\right]^{+}=1320.1\right) ; \beta-\mathrm{CD}-\mathrm{Ac}_{4}: \mathrm{m} / \mathrm{z}=1373.7\left(\left[\mathrm{C}_{54} \mathrm{H}_{78} \mathrm{O}_{39}+\mathrm{Na}\right]^{+}=1374.2\right)$. 


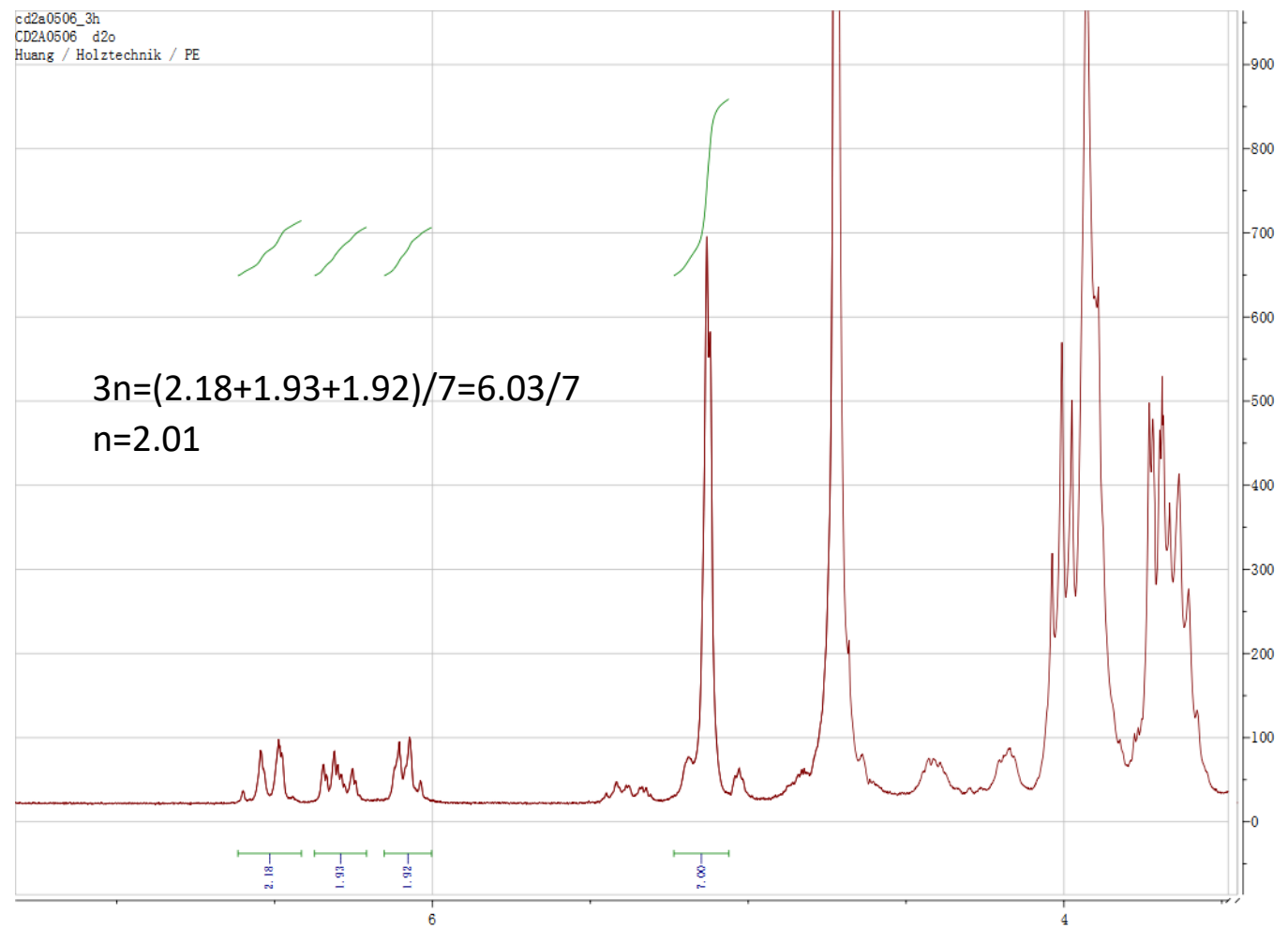

Figure S8. NMR spectrum of $\beta-\mathrm{CD}-\mathrm{Ac}_{2}\left(\right.$ in $\left.\mathrm{D}_{2} \mathrm{O}\right)$.

\section{The preparation of $\beta-C D-A c_{4.5}$}

The procedure was similar to the method of $\beta-\mathrm{CD}-\mathrm{Ac}_{2}$, but with $9.7 \mathrm{~mL}$ triethylamine $(70 \mathrm{mmol})$ and $5.5 \mathrm{~mL}$ acryloyl chloride $(67.57 \mathrm{mmol})$. The acrylate substitution ratio was calculated as 4.45 according to $1 \mathrm{H}$ NMR. Elemental analysis, calculation: C 48.36\%; H 5.78\%, found: C 49.23\%; H 6.04\%. MALDI-TOF-MS, $\beta-\mathrm{CD}-\mathrm{Ac}_{3}: \mathrm{m} / \mathrm{z}=1319.7\left(\left[\mathrm{C}_{51} \mathrm{H}_{76} \mathrm{O}_{38}+\mathrm{Na}\right]^{+}=1320.1\right)$; $\beta-C D-A c_{4}: m / z=1373.7\left(\left[\mathrm{C}_{54} \mathrm{H}_{78} \mathrm{O}_{39}+\mathrm{Na}\right]^{+}=1374.2\right) ; \beta-\mathrm{CD}-\mathrm{Ac}_{5}: \mathrm{m} / \mathrm{z}=1427.8\left(\left[\mathrm{C}_{57} \mathrm{H}_{80} \mathrm{O}_{40}+\right.\right.$ $\left.\mathrm{Na}]^{+}=1428.2\right) ; \beta-\mathrm{CD}-\mathrm{Ac}_{6}: \mathrm{m} / \mathrm{z}=1482.9\left(\left[\mathrm{C}_{60} \mathrm{H}_{82} \mathrm{O}_{41}+\mathrm{Na}\right]^{+}=1482.3\right)$. 


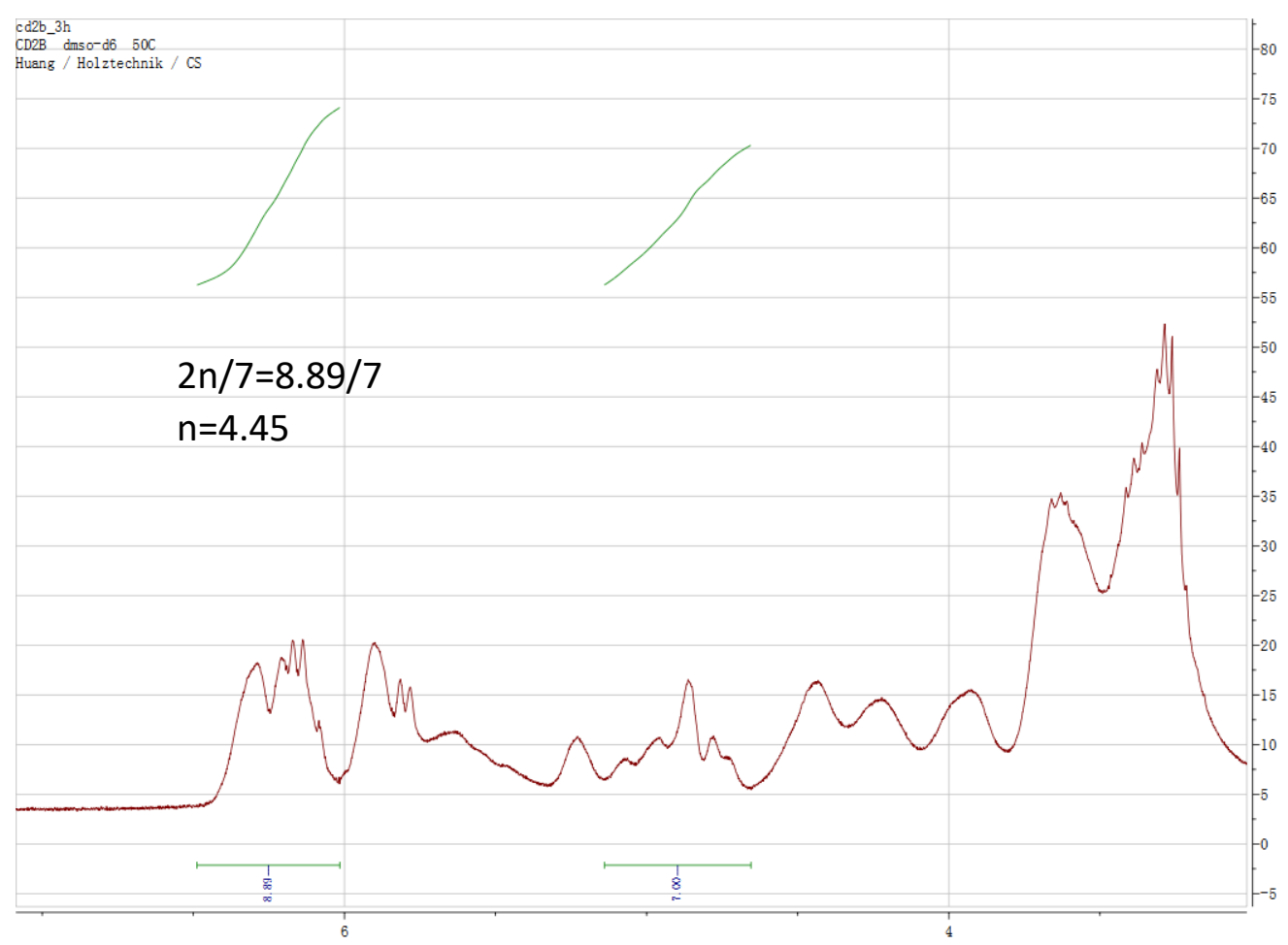

Figure S9. NMR spectrum of $\beta-\mathrm{CD}-\mathrm{Ac}_{4.5}$ (in DMSO-d6).

\section{The preparation of dynamic composite hydrogels}

The hydrogels were prepared by following the procedure in Figure S8, and the important recipes can be found in Table S1. Briefly, the pre-organization of CNC-ADA and $\beta-C D-A c_{x}$ was performed in the water and the molar ratios of $\beta-C D-A c_{x} / A D A$ were $8: 1(x=1$ or 2$)$ or $3: 1$ (for $\mathrm{x}=4.5$ ). The binding process was performed with $1.5 \mathrm{wt} \% \mathrm{CNC}-\mathrm{ADA}$ in water. The unbound $\beta-C D-A c_{x}$ was separated via repeated centrifugation. The amounts of $\beta-C D-A c_{x}$ in $\beta-$ $\mathrm{CD}-\mathrm{Ac}_{\mathrm{x}} / \mathrm{CNC}-\mathrm{ADA}$ were measured by comparing the dry weight before and after the preorganization. The purified dynamic nano-crosslinkers were mixed with AAm and polymerized to form hydrogels, where ammonium persulfate (APS, 20mM)/tetramethyethylenediamine (TEMED, $20 \mathrm{mM}$ ) were applied as initiator. To get fully swollen hydrogels, the as prepared samples were immersed in water until no weight changes. 


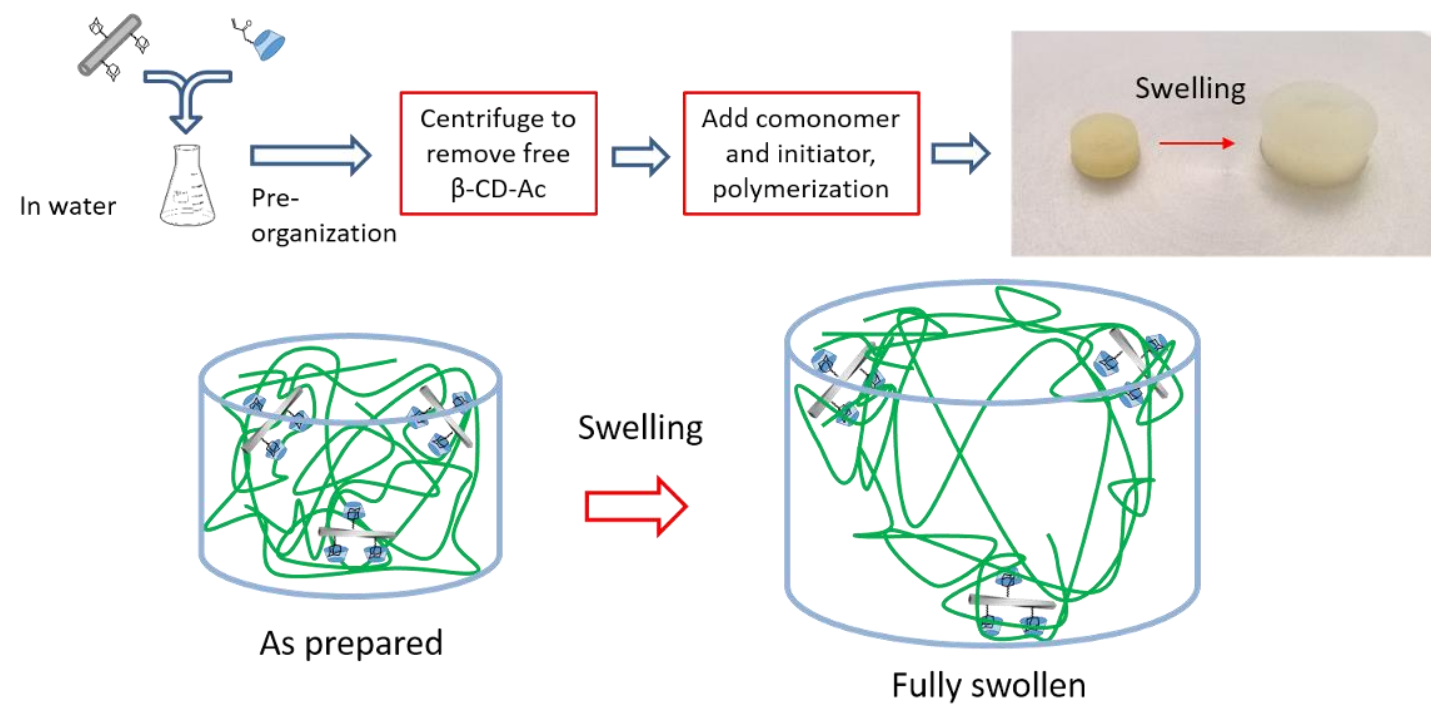

Figure S10. Schematic representation for the preparation of hydrogels.

\section{Mechanical strength tests}

The rheological properties of the dynamic composite hydrogels were investigated with a stresscontrolled bulk rheometer (Anton Paar MCR501) by using a $25 \mathrm{~mm}$ parallel plate set-up. In each test, $500 \mu \mathrm{L}$ hydrogel of each sample was prepared in advance with a thickness of $1 \mathrm{~mm}$. G', G' and tan (delta) were recorded. All the rheology measurements were carried out at room temperature $\left(23^{\circ} \mathrm{C}\right)$.

Compressive and tensile tests were performed with Z3 micro tensile test machine from GripEngineering Thümler GmbH. The cylindrical hydrogel samples (about $9.5 \mathrm{~mm}$ in diameter and $3 \mathrm{~mm}$ in height) were prepared in advance, and compressive strain rate was set at $30 \%$ per min. The rectangular specimens for tensile tests (about $17 \mathrm{~mm}$ in length, $8-9 \mathrm{~mm}$ in width and $3 \mathrm{~mm}$ in thickness) were prepared and the distance between clamps was set around $2 \mathrm{~mm}$. The default tensile speed was $1 \mathrm{~mm} / \mathrm{min}$. The compression and tensile tests were performed at $21^{\circ} \mathrm{C}$, and a relative humidity of $65 \%$. For as prepared hydrogels, the specimens were stored in the small sealed bottle during the long interval, while the fully swollen hydrogels stored in $\mathrm{H}_{2} \mathrm{O}$. For prenotched hydrogels utilized in tensile tests, the length of reset crack was cut by blade and about $30 \%$ of the specimen width. 
Table S1. Recipes of dynamic composite hydrogels and the control groups

\begin{tabular}{|c|c|c|c|c|}
\hline Name & $\begin{array}{l}\text { CNC- } \\
\text { ADA } \\
(\mathrm{wt} \%)\end{array}$ & $\begin{array}{l}\text { AAm } \\
(w t \%)\end{array}$ & $\begin{array}{l}\beta-C D-A c_{x} \\
(w t \%)\end{array}$ & $\begin{array}{l}\text { Acrylate density of } \\
\text { CNC-ADA/ } \beta-C D-A c_{x}\end{array}$ \\
\hline $\mathrm{DCH} 1 / 20 / 1^{\mathrm{a}}$ & 1 & 20 & 0.67 & $0.34 \mathrm{mmol} / \mathrm{g}$ \\
\hline DCH2/20/1 & 2 & 20 & 1.34 & $0.34 \mathrm{mmol} / \mathrm{g}$ \\
\hline DCH4/20/1 & 4 & 20 & 2.68 & $0.34 \mathrm{mmol} / \mathrm{g}$ \\
\hline DCH4/10/1 & 4 & 10 & 2.68 & $0.34 \mathrm{mmol} / \mathrm{g}$ \\
\hline $\mathrm{DCH} 4 / 5 / 1$ & 4 & 5 & 2.68 & $0.34 \mathrm{mmol} / \mathrm{g}$ \\
\hline $\mathrm{DCH} 4 / 20 / 2$ & 4 & 20 & 1.20 & $0.37 \mathrm{mmol} / \mathrm{g}$ \\
\hline $\mathrm{DCH} / 4 / 20 / 4.5$ & 4 & 20 & 0.55 & $0.40 \mathrm{mmol} / \mathrm{g}$ \\
\hline 4/20/1-post & 4 & 20 & 2.68 & -- \\
\hline $4 / 20($ no $\beta-C D)$ & 4 & 20 & 0 & -- \\
\hline 4/20/2(ADA blocked) & 4 & 20 & $2.68^{b}$ & -- \\
\hline$\beta-\mathrm{CD}-\mathrm{Ac}_{2} / \mathrm{PAAm}$ & - & 20 & 1.2 & -- \\
\hline
\end{tabular}

a: 1,2 or $4.5: 1=\beta-C D-A c_{1} ; 2=\beta-C D-A c_{2} ; 4.5=\beta-C D-A c_{4} .5$.

b: The CNC-ADA was blocked by native $\beta-C D$ in advance, then polymerized with $\beta-C D-A c_{2}$ and AAm.

\section{Scanning electron microscopy (SEM)}

The hydrogels, both as-prepared and fully-swollen specimen, were immersed in liquid nitrogen quickly, to fix the original microstructure. Then the frozen hydrogels were lyophilized at $-40^{\circ} \mathrm{C}$ under a vacuum of $10^{-2}$ mbar. In order to see the effect of external force on the microstructures of hydrogels, equally prepared as prepared hydrogels (DCH4/20/2) and fully swollen hydrogels (DCH4/20/2-S) were pre-stretched and freeze-dried under similar conditions. The SEM images were obtained on a LEO Supra-35 High-Resolution Field Emission Scanning Electron Microscope (Carl Zeiss AG, Germany) or an EVO LS15 Scanning Electron Microscope (Carl Zeiss AG, Germany). 
a

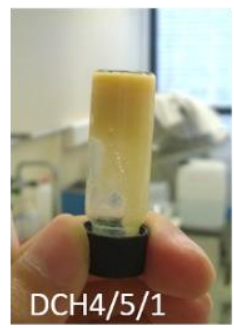

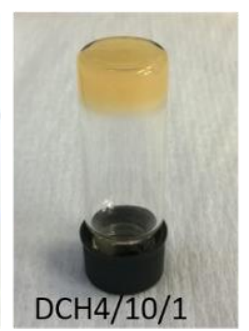

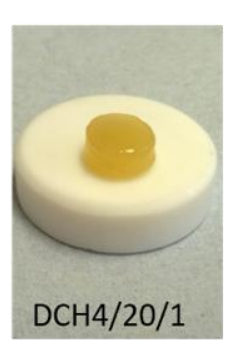

b

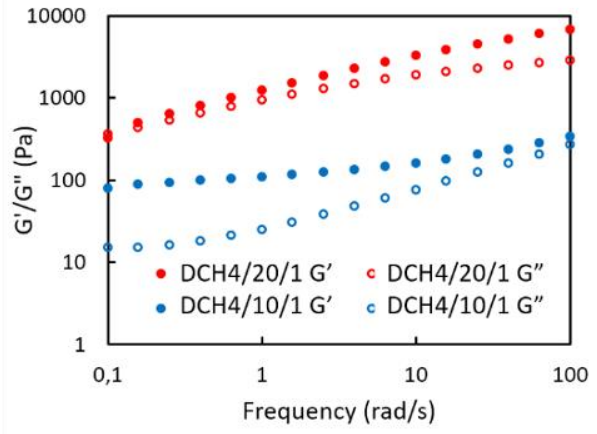

Figure S11. a) Images of as prepared hydrogels DCH4/5/1, DCH4/10/1 and DCH4/20/1; b) Rheology frequency sweeps of hydrogels DCH4/10/1 and DCH4/20/1.

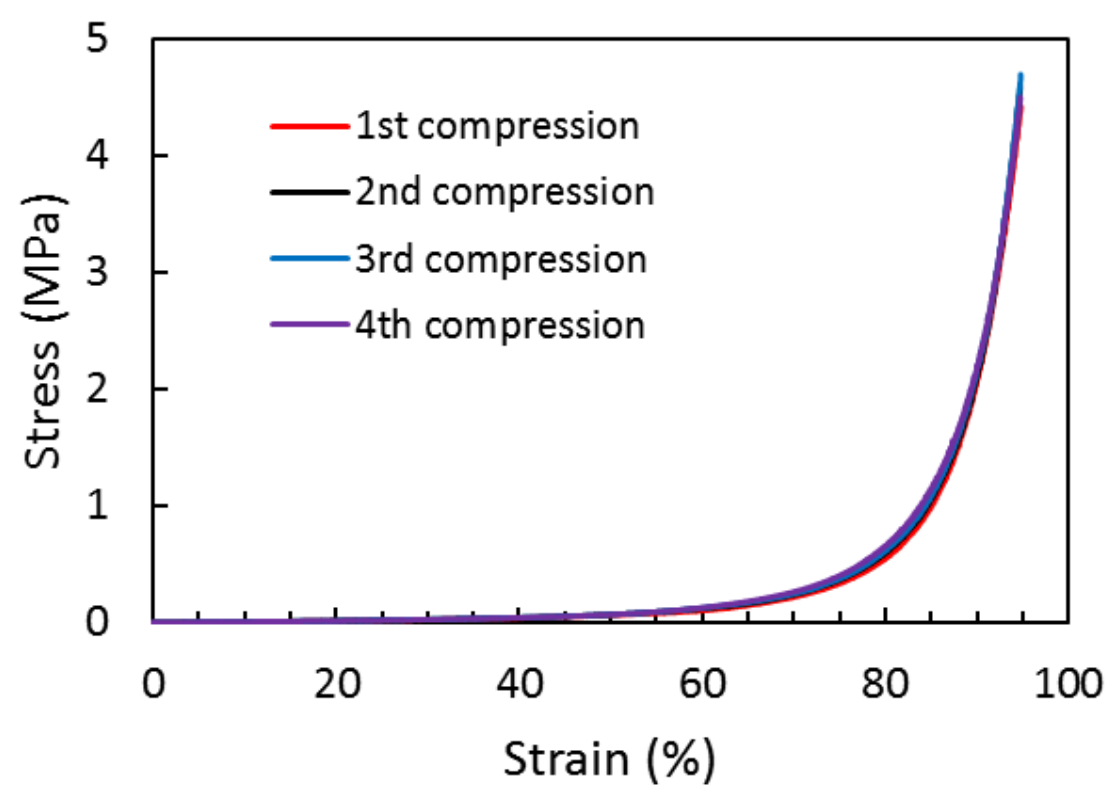

Figure S12. Repeated compression strain-stress curves of as prepared hydrogel DCH4/20/2. The compression ratio was set as $95 \%$. 

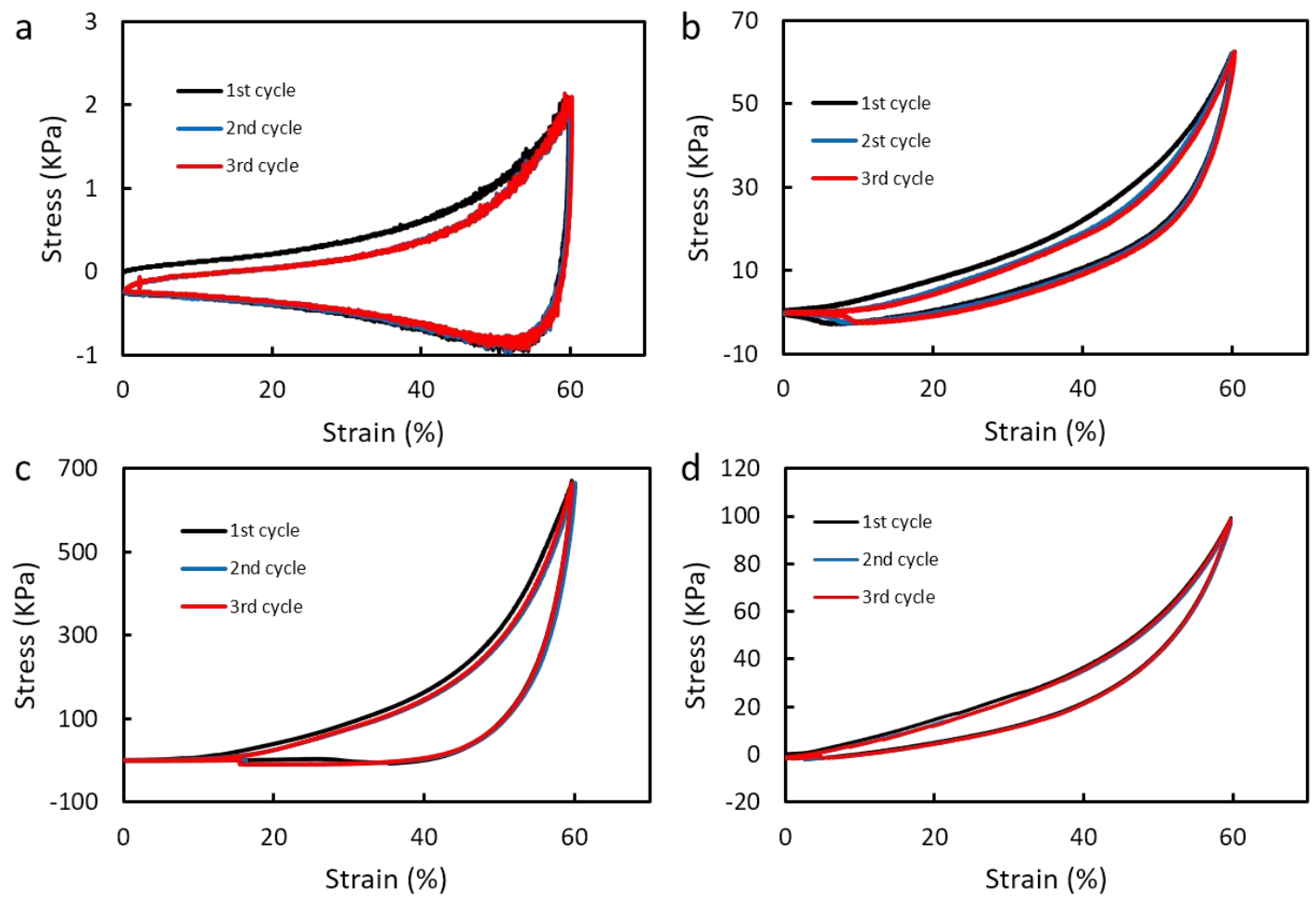

Figure S13. Continuous hysteresis compression tests of as prepared hydrogels. a) DCH4/20/1; b) $\mathrm{DCH} 4 / 20 / 2$; c) DCH/4/20/4.5; d) $\beta-\mathrm{CD}-\mathrm{Ac}_{2} / \mathrm{PAAm}$.

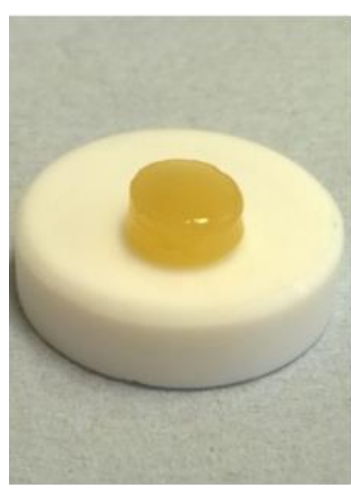

As prepared $\mathrm{DCH} 4 / 10 / 1$

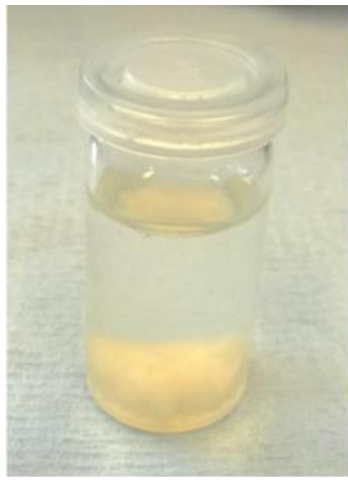

DCH4/10/1 immersed in $\mathrm{H}_{2} \mathrm{O}$ overnight

Figure S14. Photo images of as prepared DCH4/20/1 and the hydrogel DCH4/20/1 immersed in $\mathrm{H}_{2} \mathrm{O}$ for overnight. 


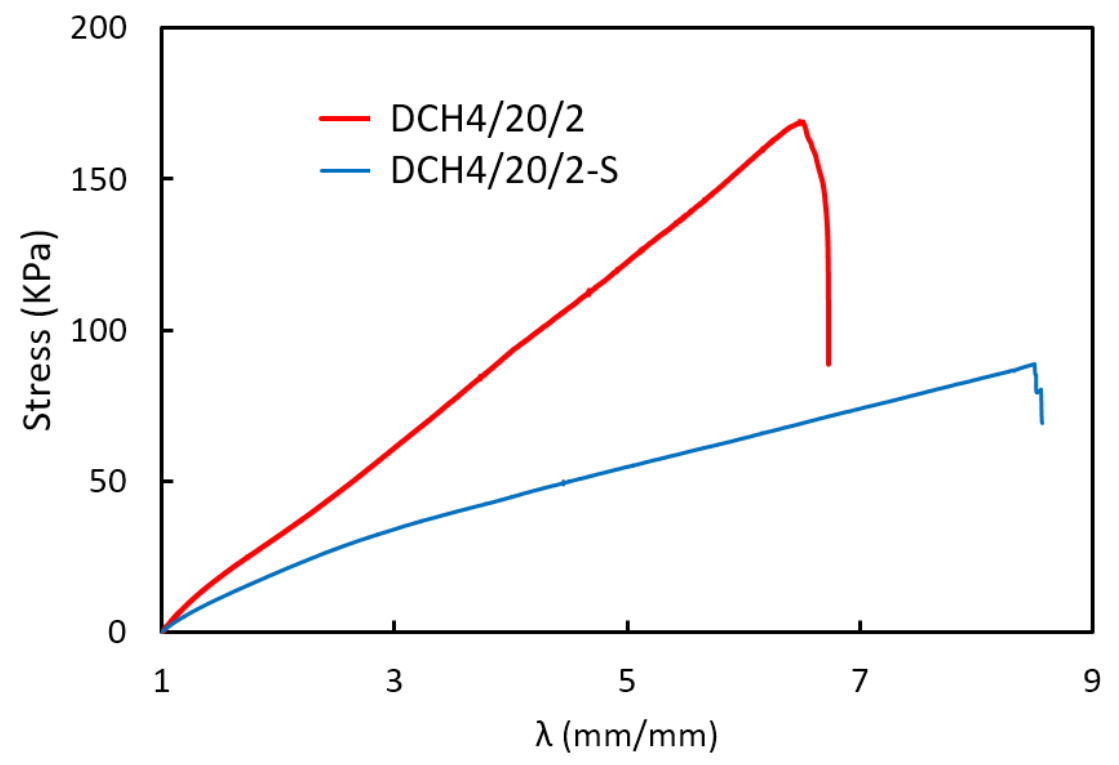

Figure S15. Tensile strain-stress curves of as prepared DCH4/20/2 and fully swollen DCH4/20/2-S.

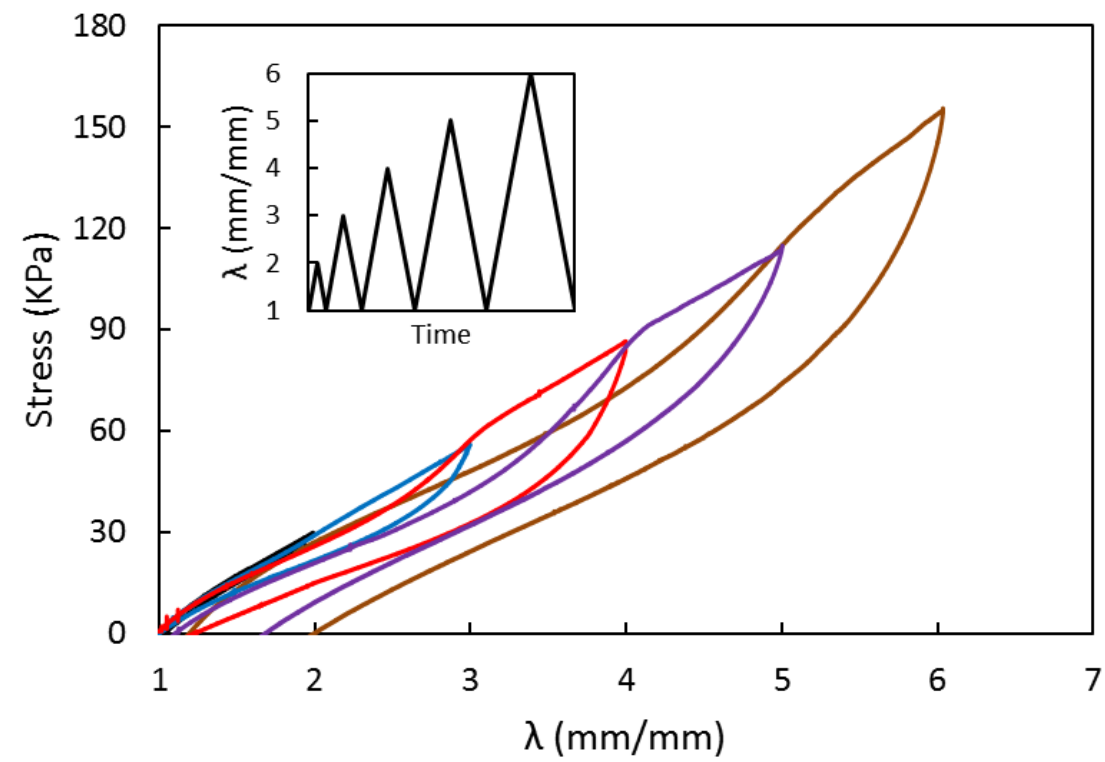

Figure S16. Step-up hysteresis tensile test strain-stress curves of as prepared DCH4/20/2. 

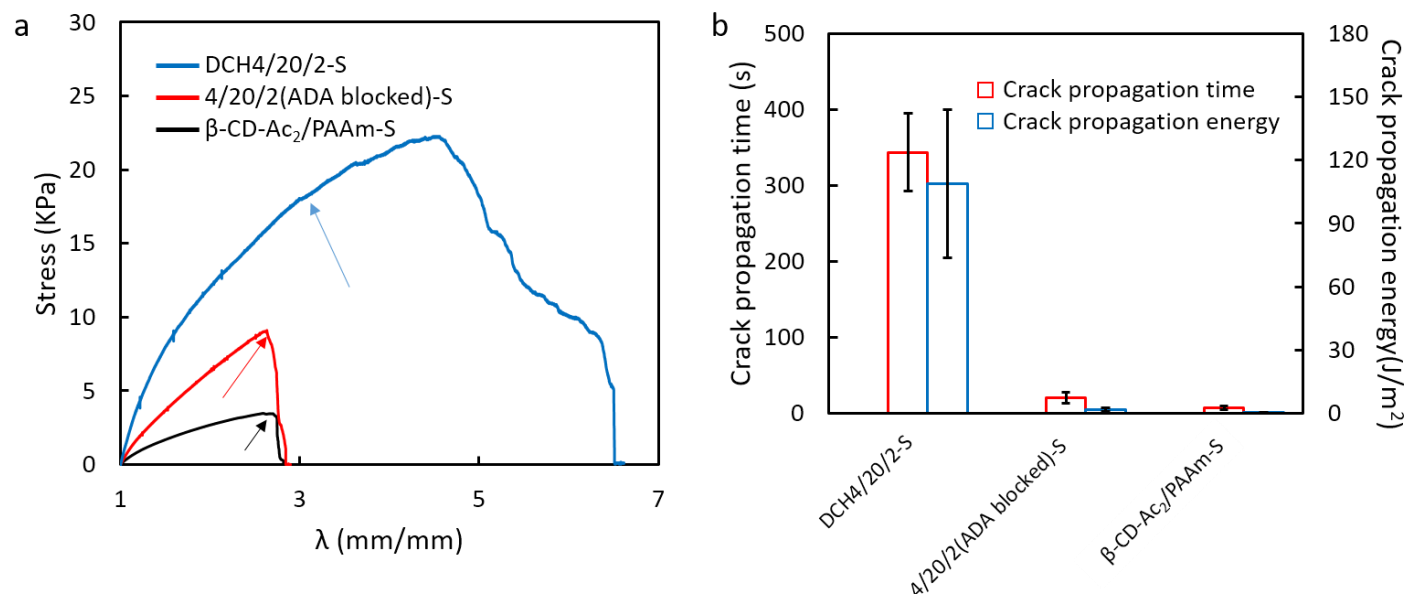

Figure S17. (a) The tensile strain-stress curves of DCH4/20/2-S, 4/20/2(ADA blocked)-S and $\beta-\mathrm{CD}-\mathrm{Ac}_{2} / \mathrm{PAAm}-\mathrm{S}$. The arrows indicate the starting point of cracks propagation. (b) The crack propagation time and crack propagation energy of DCH4/20/2-S, 4/20/2(ADA blocked)-S and $\beta-\mathrm{CD}-\mathrm{Ac}_{2} / \mathrm{PAAm}-\mathrm{S}$ as shown in (a). The crack propagation energy was calculated by the area under the strain-stress curve, from the crack starting point until the entire specimen breakup.

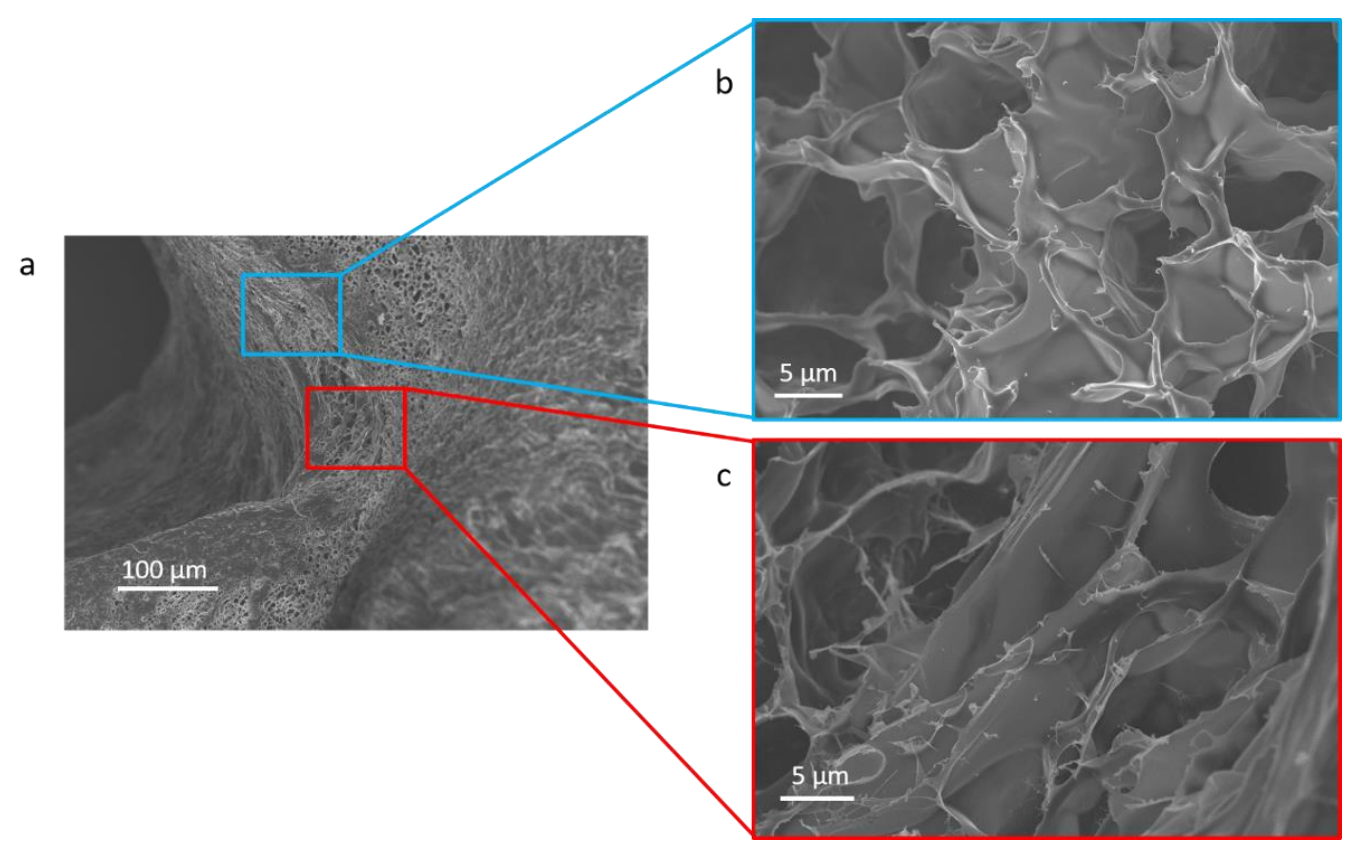

Figure S18. (a) SEM image of DCH4/20/2-S with crack. Image (b) shows the surface generated by crack propagation; Image (c) showed the topography as the last phase of the crack development. 


\section{Reference:}

[1] J. Liu, A. Plog, P. Groszewicz, L. Zhao, Y. Xu, H. Breitzke, A. Stark, R. Hoffmann, T. Gutmann, K. Zhang, G. Buntkowsky, Chem. Eur. J. 2015, 21, 12414.

[2] Y. Nakano, E. Sato, A. Matsumoto, J. Polym. Sci., Part A: Polym. Chem. 2014, 52, 2899.

[3] W. Tang, S. C. Ng, Nat. Protoc. 2008, 3, 691. 


\section{Publication 2}

\section{Dually heterogeneous hydrogels via dynamic and supramolecular crosslinks tuning discontinuous spatial ruptures}

Heqin Huang, ${ }^{1}$ Xiaojie Wang, ${ }^{1}$ Florian Rehfeldt ${ }^{2}$ and Kai Zhang*,1

${ }^{1}$ Wood Technology and Wood Chemistry, University of Goettingen, Büsgenweg 4, D-37077

Göttingen, Germany.

${ }^{2}$ Third Institute of Physics - Biophysics, Faculty of Physics, University of Goettingen, Friedrich-Hund-Platz 1, D-37077 Göttingen, Germany.

* Tel.: +49 551 394505; E-mail: kzhang1@ uni-goettingen.de

Originally published in:

ACS Sustainable Chemistry \& Engineering

American Chemical Society

DOI: 10.1021/acssuschemeng.7b04738

Received: December 15, 2017; Revised: January 30, 2018; Published online: February 1, 2018 
Abstract: Biological tissues are often highly and multiply heterogeneous in both structure and composition, but the integrity of multi-heterogeneity in artificial materials is still a big challenge. Herein, dually heterogeneous hydrogels were constructed with two distinct strategies via dynamic bonds and supramolecular crosslinks. The hydrogels showed discontinuous spatial ruptures and the mechanical behaviors of hydrogels could be tuned. The primary heterogeneity resulted from non-uniform distribution of dynamic and/or static crosslinks. The presence of only primary heterogeneity within hydrogels led to uneven mechanical properties that were represented by discontinuous spatial ruptures during the stretching the hydrogel, and therefore caused the necking deformation. Further introduction of the secondary heterogeneity by incorporating anisotropic cellulose nanocrystals (CNC) into the hydrogels allowed the adjustment of the necking phenomenon. Moreover, distinct CNC with diverse surface functionalities exhibited different effects: the "active" CNC with surfaceattached dynamic bonds retarded the necking propagation, while the "neutral" CNC without further surface-modification promoted the extension of necking points. Thus, the regulation of deformation and fracture mode of hydrogels was achieved by the synergy of dually heterogeneous structure.

Keywords: hydrogels, heterogeneous structure, necking deformation, nanocomposite, cellulose nanocrystals

\section{Introduction}

Hydrogels as highly hydrated three dimensional polymeric networks have great potential for a broad spectrum of applications ranging from tissue engineering through drug delivery systems to soft devices. ${ }^{1,2}$ In the last few years, inspired by the strong and multi-functional hydrogels in biological tissue, multi-scale and multi-mechanism design were widely used in the construction of novel artificial hydrogels and largely broaden their application in load bearing environments and complex biology systems. ${ }^{3}$

Because of the sophisticated interaction and organization, heterogeneities in composition and structure were introduced into these advanced hydrogels and uncovered many unexpected 
phenomena, which have never been found in the conventional homogeneous hydrogels. ${ }^{4}$ For example, benefit from the highly heterogeneous structures or crosslinking mechanisms, interpenetrated double-network hydrogels, ${ }^{5-8}$ nano/macro/meso-composite hydrogels, ${ }^{9-16}$ and hybrid physical/chemical crosslinked hydrogels ${ }^{17-19}$ not only maintained the advantages of hydrogels but also achieved excellent mechanical properties that were comparable to elastomer or even steels. Rather than reinforced the mechanical properties, the heterogeneous design could also change the deformation mode of hydrogels. Necking as a deformation mode normally found in metal and plastic, was reported in the highly heterogeneous hydrogels. In the double-network hydrogels, ${ }^{20-22}$ the necking is considered to be attributed to the fragmentation of the dense and brittle first network, while the flexible second network maintains the physical integrity. The necking was also found in the hydrogels constructed by polymer chains and layered double hydroxide nanosheets that were crosslinked by weak interactions. ${ }^{23}$ Due to various polymer chain lengths and their different distributions within the hydrogels with diverse crosslinking with nanosheets, the sequential detachment of polymer chains non-uniformly dissipated stored energy and led to a transiently unstable necking phenomenon.

Even though the heterogeneous design was widely applied for the construction of hydrogels, the hydrogels with multi-heterogeneities have rarely been investigated. ${ }^{24,25}$ The synergism or competition of multi-heterogeneities regarding their effects on the mechanical properties of materials, in particular hydrogels, is therefore largely unknown.

Herein, a group of dually heterogeneous hydrogels was constructed by two independent strategies. Firstly, the primary heterogeneity of hydrogels was caused by distinct distributions of dynamic and/or covalent crosslinking of polyacrylamide. With solely primary heterogeneous structure, necking deformation was observed in these hydrogels because of the discontinuous spatial ruptures under stress. In addition to heterogeneous crosslinking mechanism contributing to the primary heterogeneity, supramolecular anisotropic $\mathrm{CNC}$ were introduced into hydrogels and resulted in the secondary structural heterogeneity. Depending on the surface functionalities, carboxyl modified CNC (neutral CNC) and phenylboronic acid modified CNC (active CNC- 
PBA) could effectively regulate the necking deformation of dually heterogeneous hydrogels and thus tune their mechanical properties.

\section{Experimental section}

\section{Materials}

Microcrystalline cellulose (MCC), 2,2,6,6-tetramethylpiperidine 1-oxyl (TEMPO), cysteamine, 3-vinylphenylboronic acid, 3-(acrylamido)phenylboronic acid (PBAAM), methacrylic anhydride, sodium hydrochloride, phosphotungstic acid hydrate, 2,4,6-trimethylbenzoyl chloride and 2,2-dimethoxy-2-phenylacetophenone (DMAP) were purchased from SigmaAldrich (USA). Dopamine hydrochloride, dimethyl phenylphosphonite, 2-butanone were obtained from Alfa Aesar (USA). Acrylamide (AM), sodium sulfate anhydrous, di-sodium tetraborate and 1,4-dithiothreitol (DTT) were bought from Merck Millipore (USA). Carbonyldiimidazole (CDI) and sodium hydroxide were ordered from VWR (Germany). Sodium bicarbonate was bought from TH. Geyer (Germany). Hydrochloric acid (37\%) was obtained from AppliChem (Germany). The organic solution, including N,Ndimethylformamide (DMF), tetrahydrofuran (THF), ethyl acetate, n-hexane and acetone were all purchased from TH. Geyer (Germany). Deionized water utilized throughout all experiments was purified from a Millipore system.

\section{Preparation of CNC and CNC-PBA}

CNC was prepared with TEMPO-mediated oxidation of MCC according to the previous report. ${ }^{26}$ To remove large particles after the synthesis, the purified CNC (about $6 \mathrm{mg} / \mathrm{mL}$ ) was stored in the fridge at $4^{\circ} \mathrm{C}$ overnight. Then, the precipitated large particles were removed and the upper stable suspension was collected and stored in fridge until use. CNC was repeatedly washed with acetone and DMF, then $300 \mathrm{mg}$ CNC was redispersed in $15 \mathrm{~mL}$ DMF. Carboxyl groups on the CNC surface were activated by CDI (140 mg, $0.86 \mathrm{mmol})$ for $30 \mathrm{~min}$. Then, cysteamine (174 mg, $2.3 \mathrm{mmol}$ ) was added to CNC suspension. The amidation reaction was performed at $80^{\circ} \mathrm{C}$ under $\mathrm{N}_{2}$ protection for $48 \mathrm{~h}$. The resulted $\mathrm{CNC}-\mathrm{SH}$ was then washed with DMF for three times. The PBA was immobilized on the surface of CNC-SH via thiol-ene click 
reaction. $150 \mathrm{mg}$ washed CNC-SH was redispersed in $15 \mathrm{~mL}$ dry DMF, before 3vinylphenylboronic acid (162 mg, $1.1 \mathrm{mmol}$ ) and DMAP (30 mg, $0.12 \mathrm{mmol}$ ) were added. The immobilization was achieved by exposing to UV irradiation for 18 hours. The CNC-PBA was washed gradually to acetone and eventually to water. The content of PBA on CNC-PBA was evaluated by elemental analysis.

\section{Preparation of hydrogels}

Hydrogels in the present work were prepared in transparent poly(methyl methacrylate) molds via UV-initiated radical polymerization (Fig. 1). Glass slides were used as lid to minimize the water loss. In the alkaline aqueous solution, PBAAM and dopamine methacrylate (DMA) selfassembled as PBA/catechol complexes with two polymerizable tails, and acted as dynamic crosslinkers. AM with a constant concentration of $2 \mathrm{~mol} / \mathrm{L}$ and lithium phenyl-2,4,6trimethylbenzoylphosphinate (LAP) were used in all specimens as monomer and photoinitiator, respectively (Table $\mathrm{S} 1)$. The percursors were prepared in the alkaline buffer $(\mathrm{pH}=10)$ and purged with $\mathrm{N}_{2}$ gas to remove dissolved oxygen. Subsequently, three groups of hydrogels were prepared: as-prepared non-oxidized $(\mathrm{xNO})$, top-oxidized (xTO) and fully-oxidized (xFO) hydrogels, where $\mathrm{x}$ represented the $\mathrm{x}$ mol\% of PBA/catechol complexes regarding to the amount of AM (Fig. 1). The composite hydrogels were prepared with same procedures in the presence of $\mathrm{CNC}$ or $\mathrm{CNC}-\mathrm{PBA}$.

\section{Characterization}

The rheological properties of hydrogels were investigated with a stress-controlled bulk rheometer (Anton Paar MCR501) by using a $25 \mathrm{~mm}$ parallel plate set-up. In each test, $500 \mu \mathrm{L}$ hydrogel of each sample was prepared in advance. Storage modulus $\left(G^{\prime}\right)$, loss modulus $\left(G^{\prime \prime}\right)$ and damping factor were recorded. All the rheology measurements were carried out at room temperature $\left(\sim 23^{\circ} \mathrm{C}\right)$. Tensile tests were performed with $\mathrm{Z} 3$ micro tensile test machine from Grip-Engineering Thümler GmbH. The Dumbbell shape hydrogel samples were prepared in advance, the tensile speed was $10 \mathrm{~mm} / \mathrm{min}$. The tensile tests were performed at $\sim 21^{\circ} \mathrm{C}$ and a relative humidity of $65 \%$. 
The shape and size of $\mathrm{CNC}$ and CNC-PBA were characterized with transmission electron microscopy (TEM), and the specimens were prepared from its suspension in water of $0.01 \mathrm{wt} \%$. The specimen was stained by phosphotungstic acid solution ( $2 \mathrm{wt} \%$ in water, $\mathrm{pH}$ was adjusted to 7.0 by $1 \mathrm{M} \mathrm{NaOH}$ ). The TEM observation was conducted with a CM 12 Transmission Electron Microscope (Philips, Netherland). The optical microscopy observation of dried hydrogel films was performed with BX51-P Polarizing Microscope from Olympus.

\section{Results and discussion}

As shown in the Figure 1, non-oxidized hydrogels were prepared in sealed molds without the interference by air and therefore led to homogeneous dynamic crosslinks. To prepare topoxidized hydrogels with the primary structural heterogeneity, the upper surface of hydrogel precursor was exposed to air in the covered molds during the gelation. The oxygen in the air promoted the formation of dicatechol, which generated static dicatechol crosslinking within the top layer of dynamic hydrogel matrix. To prepare $1.25 \mathrm{NO}$, the PMMA mode was filled by hydrogel precursor and left no air in the mode. Therefore, 1.25TO represented the top-oxidized hydrogel with otherwise identical parameters as 1.25NO. Fully-oxidized hydrogels were prepared by aging the non-oxidized hydrogels in the air within sealed bottles for 2 days. For all these specimens, the acrylamide was used as an "inert" monomer to minimize the influence of non-specific interaction in the hydrogel. Furthermore, the high flexibility of polyacrylamide allowed large elongation range of hydrogels. The formation of dicatechol groups within $1.25 \mathrm{FO}$ and $1.25 \mathrm{TO}$ after the oxidation was confirmed by enhanced characteristic signals at $484 \mathrm{~nm}$ within UV-vis spectra (Fig. S5). This result was consistent with the optical changes of oxidized specimens (inset photos in Fig. 1). 

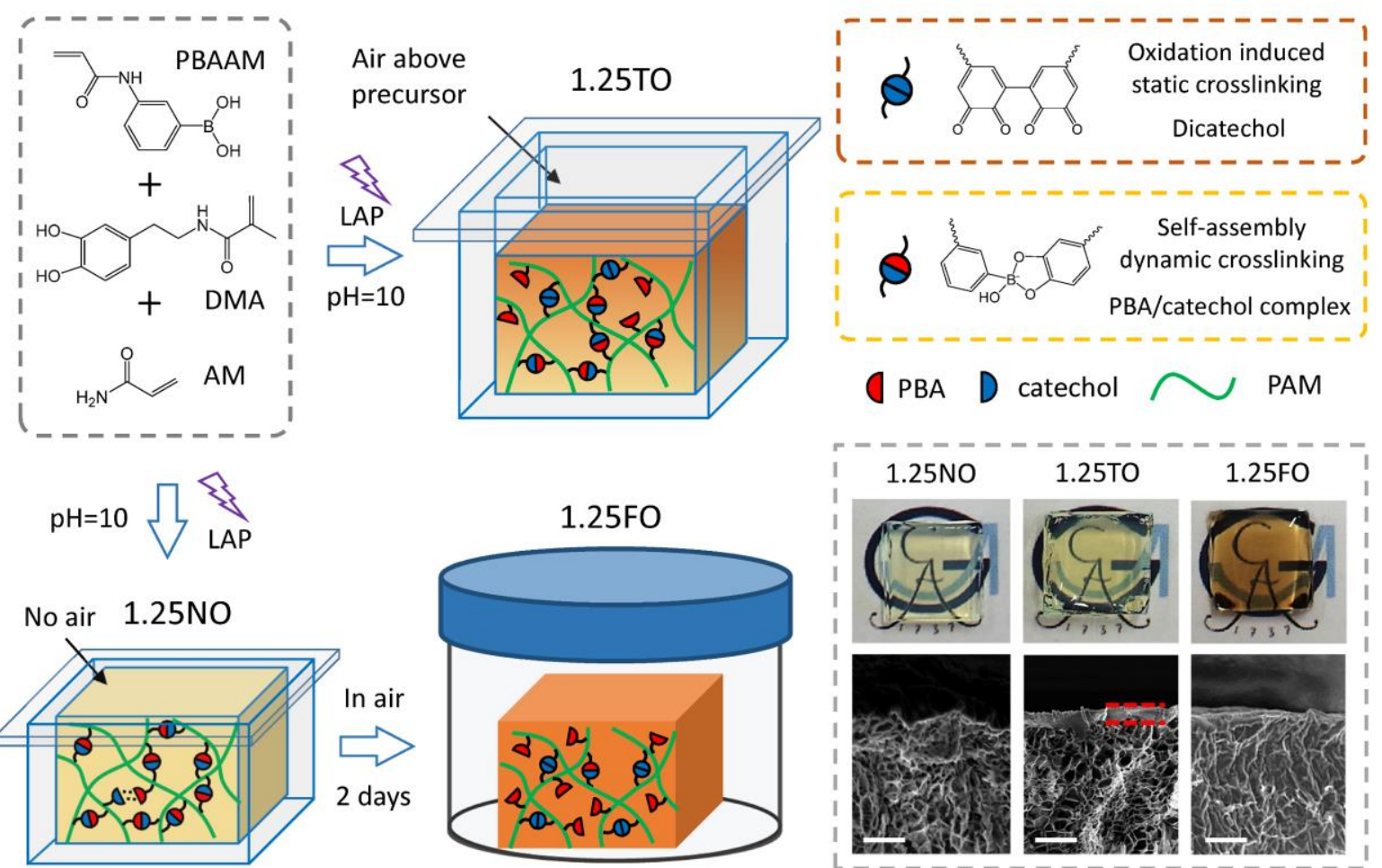

Figure 1. Schematic illustration for the preparation of top-oxidized, non-oxidized and fullyoxidized hydrogels. The inset shows the photos of as-prepared hydrogels and SEM images of freeze-dried hydrogels as $1.25 \mathrm{NO}, 1.25 \mathrm{TO}$ and 1.25FO. SEM images showed the cross section of freeze-dried hydrogels, and the oxidized top layer in 1.25TO was highlighted between red dashed lines (scale bars: $20 \mu \mathrm{m}$ ).

Furthermore, $1.25 \mathrm{NO}$ totally dissolved in the alkaline buffer solution $(\mathrm{pH}=10)$ in 2 days, because the hydrogels were loosely crosslinked by the dynamic interaction between PBA and catechol groups. In contrast, $1.25 \mathrm{FO}$ maintained its shape because of the presence of static dicatechol bonds. When the $1.25 \mathrm{TO}$ immersed in the same buffer solution, only the oxidized top layer left and the hydrogel matrix was dissolved (Fig. S6). This oxidized top layer with the thickness of about $10 \mu \mathrm{m}$ demonstrates as a dense layer in freeze-dried 1.25TO specimen (between the red dash lines in the SEM image in Fig. 1). In comparison, freeze-dried 1.25NO only showed porous structures that are characteristic for uniform hydrogels. The hydrogel matrix beneath the upper layer in $1.25 \mathrm{TO}$ had identical microstructure as the $1.25 \mathrm{NO}$. In contrast, the $1.25 \mathrm{FO}$ had an overall dense microstructure. These results together indicated that the top-oxidized hydrogels had two different crosslinking mechanisms: the whole matrix was 
stabilized by the cleavable dynamic PBA/catechol complexes, and the dicatechol groups represent static covalent crosslinking in the top layer of top-oxidized hydrogels. In comparison, non-oxidized hydrogels were only composed of the former matrix, while the fully-oxidized hydrogels were tightly crosslinked by additional dicatechol bonds.

These crosslinking mechanisms exhibited different mechanical behaviors, as demonstrated by rheology measurements of hydrogels $1.25 \mathrm{NO}$ and 1.25FO (Fig. 2A). Because of the reversible nature of PBA/catechol complex, the $\mathrm{G}^{\prime}$ and $\mathrm{G}$ " of $1.25 \mathrm{NO}$ was frequency-dependent. $1.25 \mathrm{NO}$ showed the clear fluidic feature at low frequency: $G^{\prime}<G^{\prime \prime}$. In comparison, the polymers containing solely PBAAM-AM or DMA-AM only formed viscous polymer solutions under the same condition. This fact indicated the key contribution of the PBA/catechol complexes for maintaining the hydrogel networks (Fig. S7). After the oxidation for 2 days, 1.25FO was stabilized by dicatechol static crosslinking and the $\mathrm{G}^{\prime}$ of $1.25 \mathrm{FO}$ was significantly superior to $\mathrm{G}^{\prime \prime}$ at the frequency between 0.1 and $100 \mathrm{rad} / \mathrm{s}$. The stiffness of $1.25 \mathrm{FO}$ at low frequency of $<1$ $\mathrm{rad} / \mathrm{s}$ was higher than $1.25 \mathrm{NO}$ (reflected by $\mathrm{G}^{\prime}$ ). However, static dicatechol crosslinking reduced $\mathrm{G}^{\prime}$ of $1.25 \mathrm{FO}$ at the high frequency of $>1 \mathrm{rad} / \mathrm{s}$. Since only one catechol group was needed in forming a PBA/catechol complex and each dicatechol consumed two catechol groups, the oxidation-induced conjunction actually reduced the overall crosslinking density within fully-oxidized hydrogels, thus led to lower $\mathrm{G}^{\prime}$ at higher frequency. 

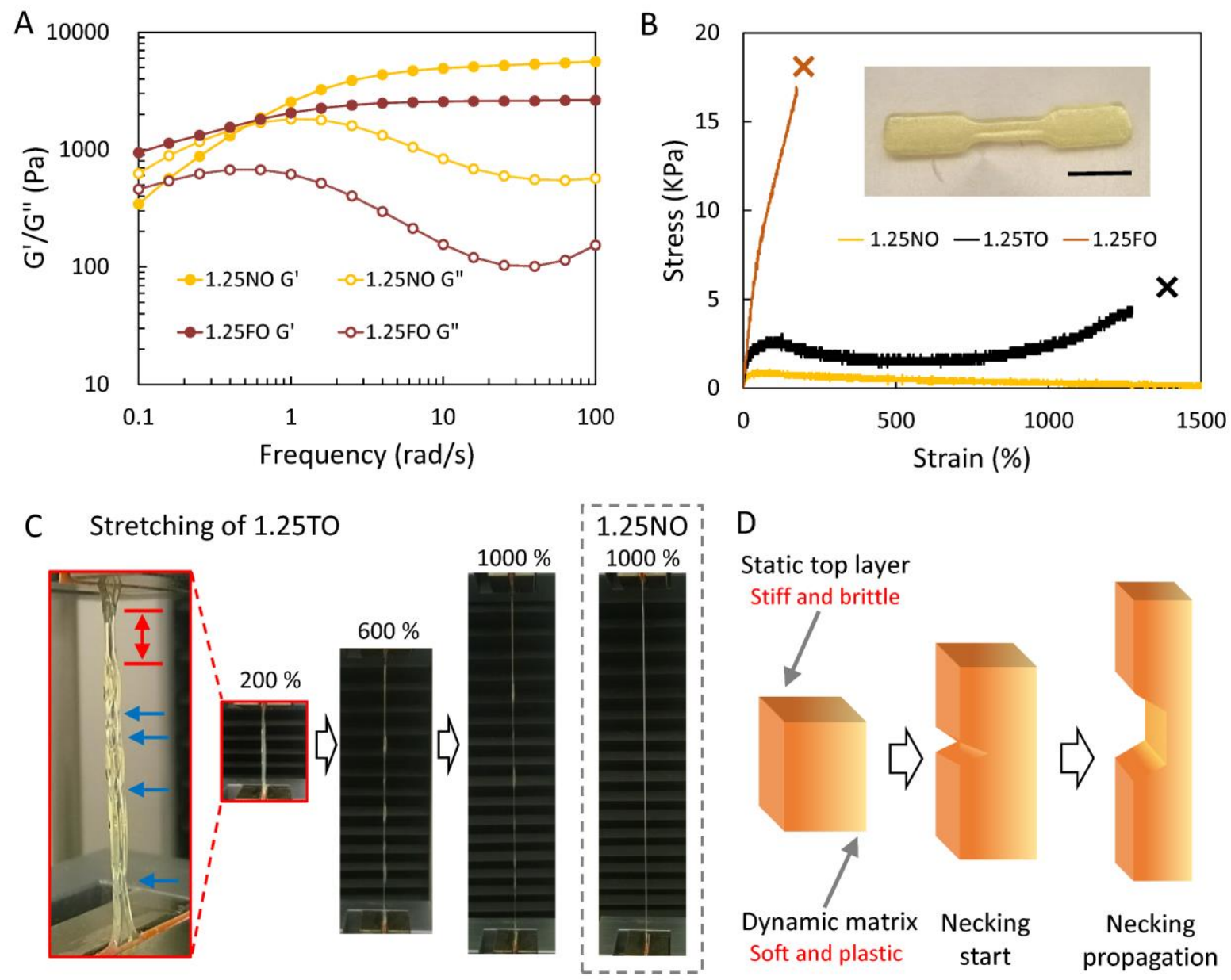

Figure 2. The heterogeneous mechanical features of top-oxidized hydrogels and their necking deformation during stretch. (A) Rheology frequency sweeps of dynamic $1.25 \mathrm{NO}$ and static 1.25FO. (B) Tensile stress-strain curves of 1.25NO, 1.25TO and 1.25FO. The inset showed the specimen (1.25TO) for tensile tests (scale bar: $10 \mathrm{~mm}$ ). (C) Photos of stretched 1.25TO and $1.25 \mathrm{NO}$. The amplified photo showed the necking phenomenon in 1.25TO. The red region indicated the propagated primary necking point, the blue arrows showed the secondary necking points. (D) Schematic illustration for the necking formation and propagation in 1.25TO.

These different mechanical behaviors were also representative in the corresponding hydrogels containing solely such crosslinks or a mixture of them. As demonstrated by the tensile tests of hydrogels performed on dumbbell-shaped specimens (inset of Fig. 2B), the hydrogel 1.25NO was very soft and plastic: after a yield point, the stress needed for further stretching was approaching zero. The stretched $1.25 \mathrm{NO}$ would not break until the end of the tensile test 
machine $(>2000 \%)$. In the present work, only the first $1500 \%$ deformation was analyzed to minimize the influence of water loss during the long time measurements. In contrast, the $1.25 \mathrm{FO}$ was elastic and brittle and broke at about 300\% elongation. The contribution of both crosslinking mechanisms with distinct distributions within hydrogels was found in the 1.25TO: after a yielding point at $\sim 100 \%$ elongation and a softening region roughly from $200 \%$ to $600 \%$, the hydrogel ruptured at about $1300 \%$ elongation after a long hardening process. Interestingly, the necking phenomenon appeared during the stretching of $1.25 \mathrm{TO}$, while $1.25 \mathrm{NO}$ deformed uniformly (Fig. 2C). During the stretching with the elongation ratio as early as of $100-150 \%$, multiple necking points appeared. The non-necking zones and necking zones were both extend during the further stretching, but the necking zones apparently undertook more deformation. The propagated primary necking points (red region) and secondary necking points (blue arrows) were highlighted in the amplified photo of $200 \%$ stretched 1.25 TO. The necking maintained and extended during further stretching at higher elongation ratios of $600 \%$ and $1000 \%$.

This necking phenomenon in $1.25 \mathrm{TO}$ was owing to the stepwise and discontinuous ruptures of the oxidized static top layer (Fig. 2D). With totally distinct mechanical properties than dynamic matrix (Fig. 2A), the static top layer that was covalently crosslinked broke earlier under the same strain (about 150\%). Once a few sites within the top layer broke, further deformation was more localized in the soft and plastic matrix that could undergo much longer stretching. The elongation of non-necking zones was largely limited by the static top layer, and the secondary necking points appeared when the non-necking zones reached the ultimate strain of static top layer. The extension of non-necking zone also drove the hardening process during further stretching (Fig. 2B). It is noteworthy that in the classic necking deformation, the necking zones would spread to the whole specimens, and form a uniform thin and soft zone. This is owing to the heterogeneity of such materials were constructed in nano-scale or micro-scale, which allow the homogenization of the sacrificed crosslinks. However, in our hydrogel system, even the necking zones extended, they did not merge into one uniform zone. Because the heterogeneity of our hydrogel was constructed by distinct mechanical features in top layer and matrix, which would form broken pieces of static top layer in macro-scale. 
Thus, the non-uniform distribution of the static dicatechol bonds and dynamic PBA/catechol crosslinks within the diverse regions in hydrogels in fact led to the heterogeneity in macrostructures. Another kind of heterogeneity can be constructed by establishing supramolecular interactions between nanoparticles and polymer chains. ${ }^{27,28}$ The necking phenomenon was also observed in such nanocomposite hydrogels ${ }^{23}$ or polymers. ${ }^{29}$ To further investigate the necking behaviors in our heterogeneous hydrogels, we used anisotropic nanocellulose with two distinct surface functionalities: CNC (TEMPO-oxidized) with only carboxyl groups $(1.52 \pm 0.03 \mathrm{mmol} / \mathrm{g})$ on the surface and CNC-PBA with surface-grafted phenylboronic acid groups $(1.07 \pm 0.05 \mathrm{mmol} / \mathrm{g})$ in addition to the rest of carboxyl groups $(\sim$ $0.4 \mathrm{mmol} / \mathrm{g}$ ) (Fig. 3A and S8). As the resource of the secondary heterogeneity in microstructures, CNC and CNC-PBA were both needle-like nanocrystals with diameters $\sim 10$ $\mathrm{nm}$ and lengths $\sim 200 \mathrm{~nm}$ (TEM images in Fig. 3B). The self-assembled chiral structures of CNC were maintained in our nanocomposite hydrogels (Fig. S9).${ }^{30,31}$ As shown in photos of Fig. 3B with the hydrogels between parallel (left) or cross (middle) polarizers, 1.25TO was hardly visible between cross polarizers (enclosed by red dashes). In comparison, hydrogels containing $2 \mathrm{wt} \% \mathrm{CNC}(1.25 / \mathrm{CNC} 2 \mathrm{TO})$ showed the unique self-assembled nematic phases between cross polarizers. These structures were also obvious in hydrogels containing $2 \mathrm{wt} \%$ CNC-PBA (1.25/CNC-PBA2, bottom right photo in Fig. 3B). By using these nanoparticles with different surface functionalities in the heterogeneous hydrogels, neutral CNC with only carboxyl groups for hydrogen bond and active CNC-PBA for PBA/catechol complex, the heterogeneity within hydrogels was further amplified. 

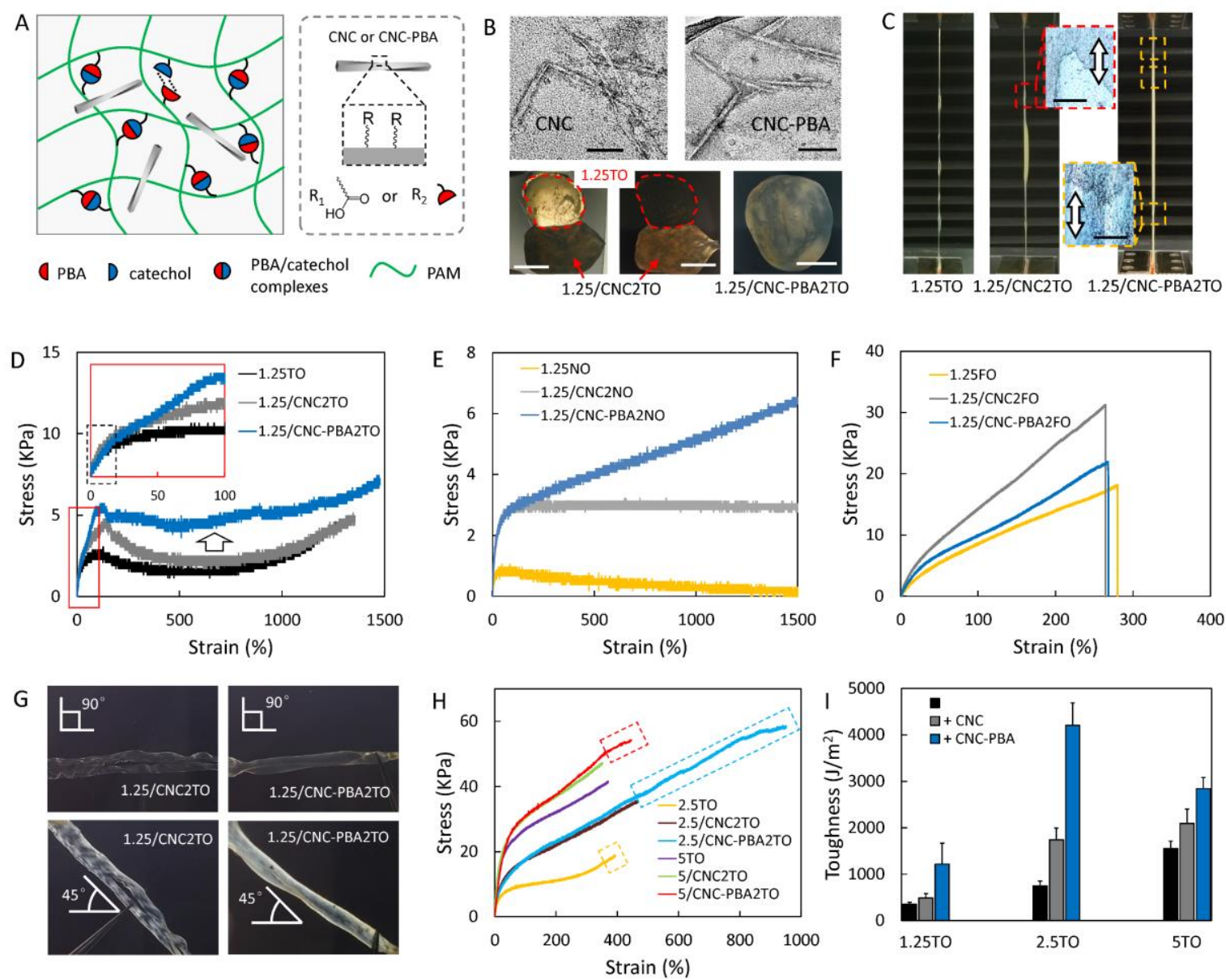

Figure 3. The necking deformation in the dually heterogeneous hydrogels. (A) Schematic illustration for the composition of hydrogels with CNC or CNC-PBA. (B) TEM images of CNC and CNC-PBA (scale bars: $100 \mathrm{~nm}$ ) and photos of 1.25TO, 1.25/CNC2TO and 1.25/CNCPBA2TO taken between parallel or perpendicular polarizers (scale bars: $2 \mathrm{~cm}$ ). (C) Photos of stretched top-oxidized hydrogels with the elongation ratios of $500 \%-600 \%$. The necking points in 1.25/CNC2TO and 1.25/CNC-PBA2TO were marked by dashed boxes. Light microscope images showed the edges of necking zone (scale bars: $200 \mu \mathrm{m}$ ). (D-F) Tensile stress-strain curves of top-oxidized hydrogels, non-oxidized hydrogels and fully-oxidized hydrogels. (G) Photos of stretched and blocked 1.25/CNC2TO and 1.25/CNC-PBA2TO between cross polarizers with diverse angles between samples and polarizers. (H) Tensile stress-strain curves of 2.5TO, 2.5/CNC2TO, 2.5/CNC-PBA2TO, 5TO, 5/CNC2TO and 5/CNC-PBA2TO. (I) The toughness of heterogeneous hydrogels, which were estimated by the areas under the corresponding stress-strain curves. 
The tensile tests demonstrated that both $\mathrm{CNC}$ and CNC-PBA reinforced the heterogeneous nanocomposite hydrogels (Fig. 3C and 3D). Interestingly, as highlighted by the amplified region of the first $100 \%$ elongation, the elastic deformation before yielding points was almost identical for $1.25 \mathrm{TO}, 1.25 / \mathrm{CNC} 2 \mathrm{TO}$ and $1.25 / \mathrm{CNC}-\mathrm{PBA} 2 \mathrm{TO}$. Therefore, they have similar elastic modulus before the yielding points, independent of the presence of CNC or CNC-PBA. This phenomenon was further confirmed by the similar G' measured from rheology frequency sweep with $1 \%$ constant strain (Fig. S10).

However, the deformation behaviors of these hydrogels after the yielding points were different, especially in the elongation regions of $>100 \%$. As illustrated in Fig. 3C, hydrogels 1.25/CNC2 TO containing CNC showed a highly non-uniform elongation in the necking zones, while the middle part of the hydrogels stayed less stretched. Compared with 1.25TO, $1.25 / \mathrm{CNC} 2 \mathrm{TO}$ underwent severe elongation and narrowing of the necking zones and rapid dropping of the stress. On the contrary, CNC-PBA effectively resisted the extension of necking points within 1.25/CNC-PBA2TO (yellow dashed boxes in Fig. 3C). At the same time, the stress dropping of 1.25/CNC-PBA2TO during the stretching was also retarded, as illustrated by its stress-strain curves in Fig. 3D. The necking zones could be clearly observed with optical microscopy (insets of Fig. $3 \mathrm{C}$ ). The edge of the necking zone was $\sim 45^{\circ}$ against the stretching direction, which was a classic phenomenon for the necking in polymers and metals. ${ }^{32,33}$

To further understand different effects of $\mathrm{CNC}$ and CNC-PBA during the necking formation and propagation, non-oxidized hydrogels $1.25 \mathrm{NO}, 1.25 / \mathrm{CNC} 2 \mathrm{NO}$ and $1.25 / \mathrm{CNC}-\mathrm{PBA} 2 \mathrm{NO}$ were investigated to exclude the interference of the primary heterogeneity in macrostructure and mechanical properties (Fig. 3E). CNC and CNC-PBA significantly improved the yield stress of hydrogels. Despite of different surface functionalities at CNC and CNC-PBA, the 1.25/CNC2NO and 1.25/CNC-PBA2NO had almost identical stress-strain curves before yielding points. Therefore, the reinforcement during the elastic deformation was not driven by specific polymer-nanoparticle interaction, but mainly because of the superior mechanical properties of $\mathrm{CNC}$ or $\mathrm{CNC}-\mathrm{PBA}$ and their orientation along the axial tensile. ${ }^{34}$ However, after the yielding point, the CNC-PBA showed significantly greater reinforcement along the plastic 
deformation up to $1500 \%$ elongation. In contrast, $1.25 / \mathrm{CNC} 2 \mathrm{NO}$ required even slightly less stress during further elongation of up to $1500 \%$. Therefore, rather than forming the composite microstructures, $\mathrm{CNC}$ and $\mathrm{CNC}-\mathrm{PBA}$ also acted as heterogeneous nano-crosslinkers in the hydrogel networks.

Similar as the fully-oxidized hydrogels $1.25 \mathrm{FO}$, fully-oxidized hydrogels $1.25 / \mathrm{CNC} 2 \mathrm{FO}$ and 1.25/CNC-PBA2FO only showed elastic deformation (Fig. 3F). Interestingly, CNC acted as a better reinforcing nanofillers than CNC-PBA in these statically crosslinked hydrogels. This was probably attributed to that the extra PBA from CNC-PBA may have occupied more catechol groups and thus reduced the amount of dicatechol crosslinking.

Furthermore, the uneven elongation due to the necking of $1.25 / \mathrm{CNC} 2 \mathrm{TO}$ and $1.25 / \mathrm{CNC}$ PBA2TO could be fixed and visualized thanks to the birefringence property of stretched CNC composite hydrogels (Fig. 3G). ${ }^{35}$ For this purpose, 1.25/CNC2TO and 1.25/CNC-PBA2TO were prepared as rectangular hydrogel films. Then, these hydrogel films were stretched to about $600 \%$ of their original length and dried to fix the microstructure. In the Fig. 3G, strain-induced orientation of anisotropic CNC led to a strong birefringence signal, when the stretching direction was placed $45^{\circ}$ against the two perpendicular polarizers. In stretched $1.25 / \mathrm{CNC} 2 \mathrm{TO}$ and 1.25/CNC-PBA2TO, necking zones and non-necking zones showed different brightness. This was owing to the different degree of orientation, and was originally generated by the nonuniform elongation.

The necking phenomenon was also sensitive to the crosslinking density of hydrogels, independent of the presence of CNC or CNC-PBA. Hydrogels 2.5TO and 5TO were prepared, which contained 2.5 and $5 \mathrm{~mol} \%$ of the monomer AM, respectively. According to the rheology frequency sweep tests (Fig. S11), 2.5TO and 5TO showed similar frequency-dependency as 1.25TO but with improved stiffness. The results from tensile tests were consistent with the rheology measurements (Fig. 3H), which showed improved elastic modulus for 2.5TO and 5TO. Moreover, the maximal elongation ratios of $2.5 \mathrm{TO}$ and $5 \mathrm{TO}$ decreased to $\sim 400 \%$ and $\sim 350 \%$, respectively. In $2.5 \mathrm{TO}$, the breaking of the static top layer occurred slightly earlier than overall fracture of the specimens (yellow dashed box in Fig. $3 \mathrm{H}$ ), indicated a very short necking 
process. With even higher amount of $\mathrm{PBA} /$ catechol complexes in 5TO, the necking became imperceptible.

As mentioned above, CNC and CNC-PBA affected the necking behavior in hydrogel 1.25TO. These nanofillers were also utilized to tune the mechanical properties of $2.5 \mathrm{TO}$ and 5TO. As expected, both CNC and CNC-PBA improved the yield stress of hydrogels. 2.5/CNC2TO and 2.5/CNC-PBA2TO had the almost identical stress-strain curves in the first $450 \%$ deformation, until 2.5/CNC2TO broke down. However, the 2.5/CNC-PBA2TO could be further stretched to about $950 \%$ of its original length, although the stress-strain curves fluctuated because of the ruptures of the static top layer (blue dashed box). Similar necking phenomenon was also observed in the $5 \mathrm{TO}$ and its corresponding composite specimen 5/CNC-PBA2TO (red dashed box). The active CNC-PBA improved the tolerance to the ruptures of top static layer and avoided the overall fracture during further elongation, therefore the toughness of composite hydrogels increased significantly (Fig. 3I).

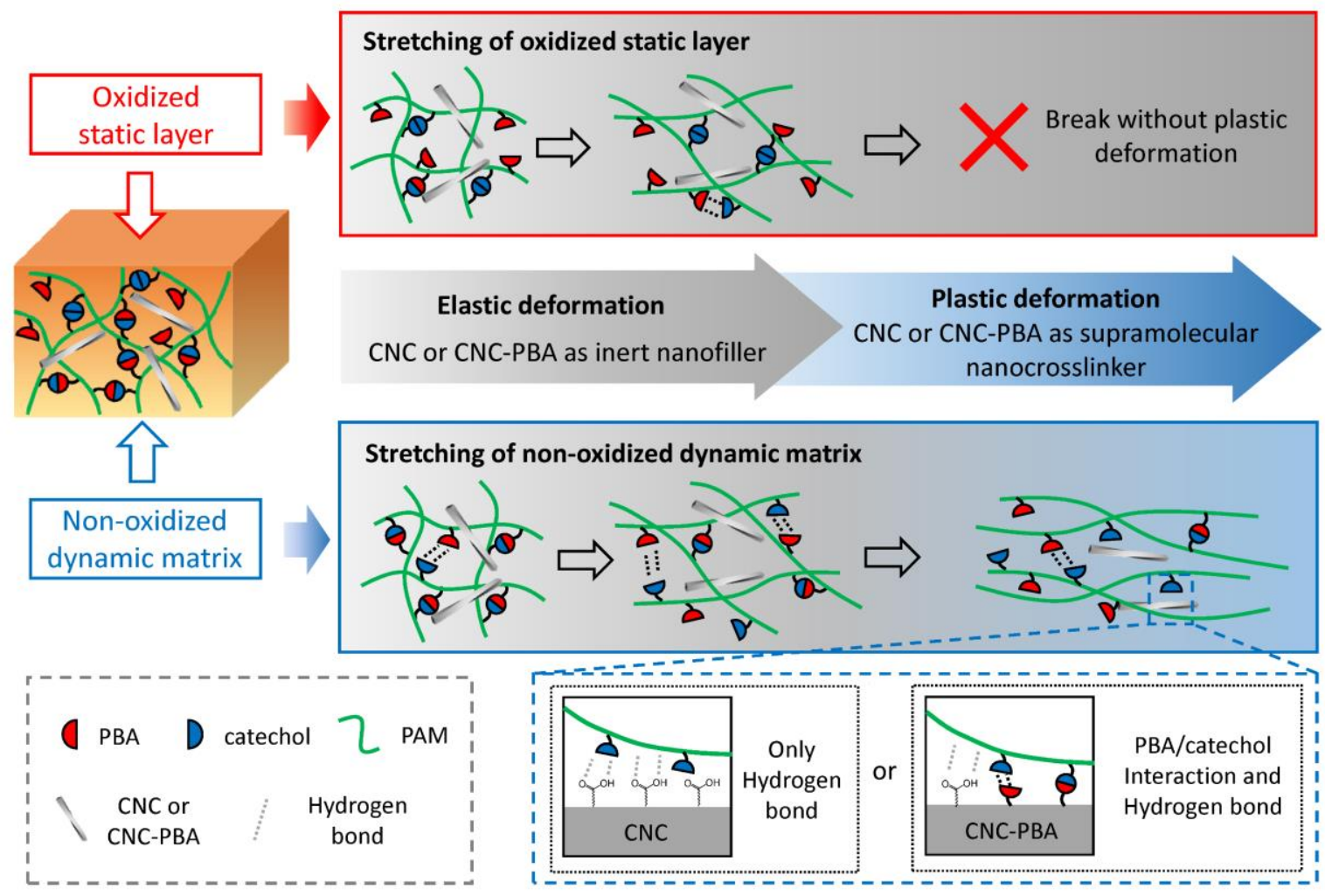

Figure 4. Diverse $\mathrm{CNC}$ drive distinct reinforcement mechanisms in static and dynamic 
hydrogels. Schematic illustration showing diverse deformation modes of oxidized static top layer and non-oxidized dynamic matrix, respectively. Neutral CNC and active CNC-PBA with distinct surface functionalities have different dynamic interactions with surrounding polymers during the long-distance plastic deformation.

Based on the results above, active $\mathrm{CNC}-\mathrm{PBA}$ and neutral $\mathrm{CNC}$ performed various reinforcing mechanisms in hydrogels containing distinct crosslinking environments (Fig. 4). During the stretching of top-oxidized hydrogels, the plastic deformation of non-oxidized dynamic hydrogel dominated in the necking zones, while the PBA/catechol complexes continuously and rapidly dissociated and associated to maintain the physical integrity. Therefore, the active CNC-PBA was able to participate in the dynamic crosslinking networks and strengthened the necking zones. The elastic deformation mainly occurred in the non-necking zone, while CNCPBA showed much less reinforcement in the elastic deformation, no matter in static or dynamic hydrogel matrix. This different performance of active CNC-PBA acting as an autonomous compensation mechanism narrowed the difference of mechanical stability between necking and non-necking zone, leading to retarded propagation of necking zone in whole hydrogels. In contrast, neutral CNC among the plastic deformation only had non-specific hydrogen bonds to surrounding polymer chains, so that it failed in reinforcing the plastic necking zone. However, neutral CNC effectively reinforced the static crosslinking network within the non-necking zone. Thus, in contrary to the active CNC-PBA, neutral CNC further amplified the mechanical difference between the necking and non-necking zones, and promoted the necking propagation. As well, these diverse $\mathrm{CNC}$ tuned the non-uniform mechanical properties due to their distinct interactions with hydrogel networks.

\section{Conclusions}

In summary, a novel group of heterogeneous hydrogels was prepared by combining static top layer and dynamic matrix with distinct spatial distributions, which provided the primary heterogeneity in macrostructures. During the long-distance stretching of these heterogeneous hydrogels, the discontinuous ruptures of static oxidized layer led to necking deformation. However, this necking deformation did not occur in solely dynamic or static crosslinked 
hydrogels. Furthermore, as releaser for the secondary heterogeneity, anisotropic nanosized $\mathrm{CNC}$ was introduced into the heterogeneous hydrogels. Diverse CNC with different surfacefunctionalities were involved into the hydrogel networks via distinct mechanisms. Active CNC with dynamic bonds (PBA) on surface could actively establish supramolecular interactions with other counter parts (catechol) within hydrogels, while neutral CNC with carboxyl groups could primarily form non-specific hydrogen bonds with other component within hydrogels. Consequently, the necking deformation and the mechanical properties of the dually heterogeneous hydrogels could be tuned by introducing diverse CNC. By fully utilizing the distinct reinforcing mechanisms of $\mathrm{CNC}$ with different surface-functionalities in various dynamic environments, we achieved the first strategy to regulate the necking deformation in the hydrogel system and thus adjusted their mechanical stability. Prospectively, the demonstration of the tunable necking behaviors in hydrogels should not only advance the understanding of the mechanical performance of the soft and hydrate materials, but also uncover the great potential of the multi-heterogeneous design strategy in a wide variety of practical applications in the future.

\section{Supporting information}

Other experimental procedures are provided in Supporting Information.

\section{Acknowledgements}

K.Z. thanks German Research Foundation (DFG) for the project ZH546/3-1 and Fonds der Chemischen Industrie (FCI) for the financial support. The program 'Material Science Wood' from Lower Saxony Ministry of Science and Culture, Germany, is gratefully acknowledged for the financial support. X.W. thanks China Scholarship Council (CSC) for the financial support. The authors also thank Wentao Peng, Alex Groschopp and Prof. Philipp Vana for helpful discussion.

\section{Reference and notes}

(1) Thiele, J.; Ma, Y.; Bruekers, S. M.; Ma, S.; Huck, W. T., 25th anniversary article: Designer hydrogels for cell cultures: a materials selection guide. Adv. Mater. 2014, 26 (1), 125-147. 
(2) Appel, E. A.; del Barrio, J.; Loh, X. J.; Scherman, O. A., Supramolecular polymeric hydrogels. Chem. Soc. Rev. 2012, 41 (18), 6195-6214.

(3) Zhao, X., Multi-scale multi-mechanism design of tough hydrogels: building dissipation into stretchy networks. Soft Matter 2014, 10 (5), 672-687.

(4) Zhang, Y. S.; Khademhosseini, A., Advances in engineering hydrogels. Science 2017, 356 (6337), eaaf3627.

(5) Gong, J. P., Why are double network hydrogels so tough? Soft Matter 2010, 6 (12), 25832590.

(6) Truong, V. X.; Ablett, M. P.; Richardson, S. M.; Hoyland, J. A.; Dove, A. P., Simultaneous orthogonal dual-click approach to tough, in-situ-forming hydrogels for cell encapsulation. $J$. Am. Chem. Soc. 2015, 137 (4), 1618-1622.

(7) Zhang, H. J.; Sun, T. L.; Zhang, A. K.; Ikura, Y.; Nakajima, T.; Nonoyama, T.; Kurokawa, T.; Ito, O.; Ishitobi, H.; Gong, J. P., Tough Physical Double-Network Hydrogels Based on Amphiphilic Triblock Copolymers. Adv. Mater. 2016, 28 (24), 4884-4890.

(8) Sun, J. Y.; Zhao, X.; Illeperuma, W. R.; Chaudhuri, O.; Oh, K. H.; Mooney, D. J.; Vlassak, J. J.; Suo, Z., Highly stretchable and tough hydrogels. Nature 2012, 489 (7414), 133-136.

(9) Yang, J.; Ma, M.; Zhang, X.; Xu, F., Elucidating Dynamics of Precoordinated Ionic Bridges as Sacrificial Bonds in Interpenetrating Network Hydrogels. Macromolecules 2016, 49 (11), 4340-4348.

(10)Thoniyot, P.; Tan, M. J.; Karim, A. A.; Young, D. J.; Loh, X. J., Nanoparticle-Hydrogel Composites: Concept, Design, and Applications of These Promising, Multi-Functional Materials. Adv. Sci. 2015, 2, 1400010.

(11)Janecek, E. R.; McKee, J. R.; Tan, C. S.; Nykanen, A.; Kettunen, M.; Laine, J.; Ikkala, O.; Scherman, O. A., Hybrid supramolecular and colloidal hydrogels that bridge multiple length scales. Angew. Chem. Int. Ed. 2015, 54 (18), 5383-5388.

(12)Haraguchi, K., Synthesis and properties of soft nanocomposite materials with novel organic/inorganic network structures. Polym. J. 2011, 43 (3), 223-241.

(13)Wang, Q.; Mynar, J. L.; Yoshida, M.; Lee, E.; Lee, M.; Okuro, K.; Kinbara, K.; Aida, T., High-water-content mouldable hydrogels by mixing clay and a dendritic molecular binder. 
Nature 2010, 463 (7279), 339-343.

(14)Yang, S.; Wang, J.; Tan, H.; Zeng, F.; Liu, C., Mechanically robust PEGDA-MSNs-OH nanocomposite hydrogel with hierarchical meso-macroporous structure for tissue engineering. Soft Matter 2012, 8 (34), 8981-8989.

(15)Illeperuma, W. R. K.; Sun, J.-Y.; Suo, Z.; Vlassak, J. J., Fiber-reinforced tough hydrogels. Extreme Mechanics Letters 2014, 1, 90-96.

(16)Huang, Y.; King, D. R.; Sun, T. L.; Nonoyama, T.; Kurokawa, T.; Nakajima, T.; Gong, J. P., Energy-Dissipative Matrices Enable Synergistic Toughening in Fiber Reinforced Soft Composites. Adv. Funct. Mater. 2017, 27 (9), 1605350.

(17)Wei, K.; Chen, X.; Li, R.; Feng, Q.; Bian, L., Multivalent Host-Guest Hydrogels as Fatigue-Resistant 3D Matrix for Excessive Mechanical Stimulation of Encapsulated Cells. Chem. Mater. 2017, 29 (20), 8604-8610.

(18)Wei, Q.; Xu, M.; Liao, C.; Wu, Q.; Liu, M.; Zhang, Y.; Wu, C.; Cheng, L.; Wang, Q., Printable hybrid hydrogel by dual enzymatic polymerization with superactivity. Chem. Sci. 2016, 7 (4), 2748-2752.

(19)Imran, A. B.; Esaki, K.; Gotoh, H.; Seki, T.; Ito, K.; Sakai, Y.; Takeoka, Y., Extremely stretchable thermosensitive hydrogels by introducing slide-ring polyrotaxane cross-linkers and ionic groups into the polymer network. Nat. Commun. 2014, 5, 5124.

(20)Na, Y.; Tanaka, Y.; Kawauchi, Y.; Furukawa, H.; Sumiyoshi, T.; Gong, J. P.; Osada, Y., Necking Phenomenon of Double-Network Gels. Macromolecules 2006, 39 (14), 4641-4645.

(21)Chen, Q.; Zhu, L.; Zhao, C.; Wang, Q.; Zheng, J., A robust, one-pot synthesis of highly mechanical and recoverable double network hydrogels using thermoreversible sol-gel polysaccharide. Adv. Mater. 2013, 25 (30), 4171-4176.

(22)Es-haghi, S. S.; Leonov, A. I.; Weiss, R. A., On the Necking Phenomenon in Pseudo-SemiInterpenetrating Double-Network Hydrogels. Macromolecules 2013, 46 (15), 6203-6208.

(23) Hu, Z.; Chen, G., Novel nanocomposite hydrogels consisting of layered double hydroxide with ultrahigh tensibility and hierarchical porous structure at low inorganic content. Adv. Mater. 2014, 26 (34), 5950-5956.

(24)Gladman, A. S.; Matsumoto, E. A.; Nuzzo, R. G.; Mahadevan, L.; Lewis, J. A., Biomimetic 
4D printing. Nat. Mater. 2016, 15 (4), 413-418.

(25)Liu, L.; Ghaemi, A.; Gekle, S.; Agarwal, S., One-Component Dual Actuation: Poly(NIPAM) Can Actuate to Stable 3D Forms with Reversible Size Change. Adv. Mater. 2016, 28 (44), 9792-9796.

(26)Liu, J.; Plog, A.; Groszewicz, P.; Zhao, L.; Xu, Y.; Breitzke, H.; Stark, A.; Hoffmann, R.; Gutmann, T.; Zhang, K.; Buntkowsky, G., Design of a Heterogeneous Catalyst Based on Cellulose Nanocrystals for Cyclopropanation: Synthesis and Solid-State NMR Characterization. Chem. Eur. J. 2015, 21 (35), 12414-12420.

(27)Huang, H.; Wang, Y.; Wang, X.; Rehfeldt, F.; Zhang, K., Robust Heterogeneous Hydrogels with Dynamic Nanocrystal-Polymer Interface. Macromol. Rapid Commun. 2017, 38 (12), 1600810.

(28)Haraguchi, K.; Uyama, K.; Tanimoto, H., Self-healing in nanocomposite hydrogels. Macromol. Rapid Commun. 2011, 32 (16), 1253-1258.

(29)Haraguchi, K.; Ebato, M.; Takehisa, T., Polymer-Clay Nanocomposites Exhibiting Abnormal Necking Phenomena Accompanied by Extremely Large Reversible Elongations and Excellent Transparency. Adv. Mater. 2006, 18 (17), 2250-2254.

(30)Wang, P. X.; Hamad, W. Y.; MacLachlan, M. J., Structure and transformation of tactoids in cellulose nanocrystal suspensions. Nat. Commun. 2016, 7, 11515.

(31)Usov, I.; Nystrom, G.; Adamcik, J.; Handschin, S.; Schutz, C.; Fall, A.; Bergstrom, L.; Mezzenga, R., Understanding nanocellulose chirality and structure-properties relationship at the single fibril level. Nat. Commun. 2015, 6, 7564.

(32)Hill, R., On discontinuous plastic states, with special reference to localized necking in thin sheets. J. Mech. Phys. Solids 1952, 1 (1), 19-30.

(33) Stören, S.; Rice, J. R., Localized necking in thin sheets. J. Mech. Phys. Solids 1975, 23 (6), 421-441.

(34)Habibi, Y., Key advances in the chemical modification of nanocelluloses. Chem. Soc. Rev. 2014, 43 (5), 1519-1542.

(35)Tummala, G. K.; Joffre, T.; Rojas, R.; Persson, C.; Mihranyan, A., Strain-induced stiffening of nanocellulose-reinforced poly(vinyl alcohol) hydrogels mimicking collagenous 
soft tissues. Soft Matter 2017, 13 (21), 3936-3945. 


\section{Publication 2 (Supporting information)}

\section{Dually heterogeneous hydrogels via dynamic and supramolecular crosslinks tuning discontinuous spatial ruptures}

Heqin Huang, Xiaojie Wang, Florian Rehfeldt and Kai Zhang*

\section{Synthesis of dopamine methylacrylate (DMA)}

Dopamine methacrylamide (DMA) was prepared with a published method. ${ }^{1} 5 \mathrm{~g}$ of sodium borate and $2 \mathrm{~g}$ of sodium bicarbonate were dissolved in $50 \mathrm{~mL}$ of DI water and purged by $\mathrm{N}_{2}$ gas. Then $2.5 \mathrm{~g}$ dopamine hydrochloride was added and the solution was cooled in ice bath. $2.35 \mathrm{~mL}$ of methacrylate anhydride was dispersed in $15 \mathrm{~mL}$ of THF and was added to cooled solution. After 3 hours reaction at $4^{\circ} \mathrm{C}$, the reaction was further carried out at room temperature overnight. The whole reaction was protected by with $\mathrm{N}_{2}$ gas. The reaction mixture was washed with $25 \mathrm{~mL}$ of ethyl acetate, then the $\mathrm{pH}$ value was adjusted to 2 with $5 \mathrm{M} \mathrm{HCl}$ solution. Then, $3 \times 25 \mathrm{~mL}$ of ethyl acetate was used to extract the product. The combined organic layers were dried overnight under sodium sulfate anhydrous and concentrated to $\sim 20 \mathrm{~mL}$. Then, $150 \mathrm{~mL}$ of n-hexane was slowly added to precipitate the product. The final product was dried in the vacuum oven as grey powder.

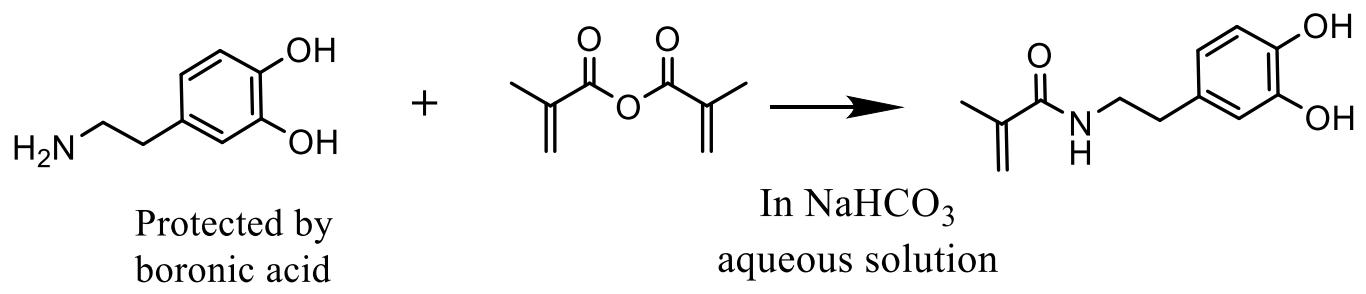

Figure S1. Schematic illustration for the synthesis of dopamine methacrylate. 


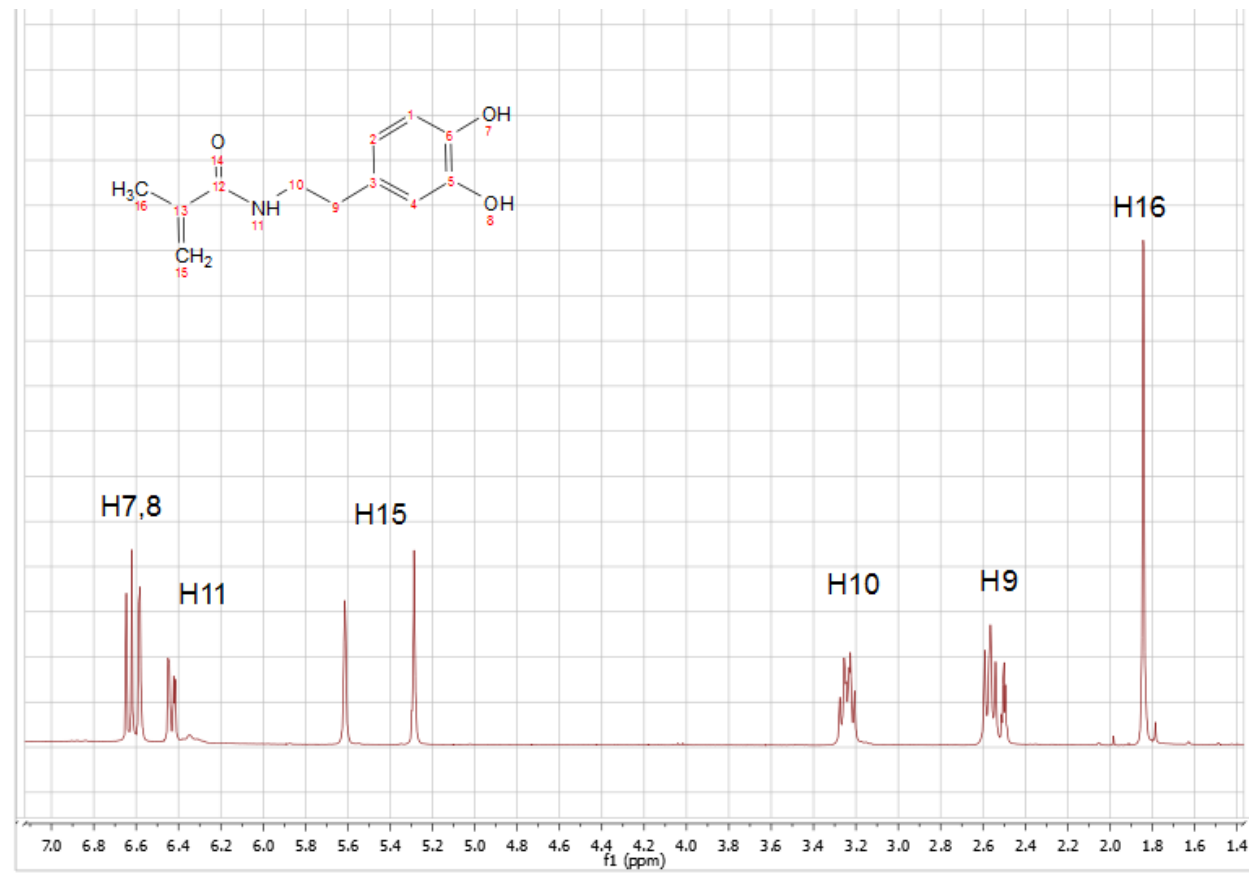

Figure S2. ${ }^{1} \mathrm{H}$ NMR spectrum of dopamine methacrylate in DMSO-d6.

\section{Synthesis of photoinitiator lithium acylphosphinate (LAP)}

The photoinitiator LAP was prepared according to reported method. ${ }^{2} 1.6 \mathrm{~g}$ 2,4,6trimethylbenzoyl chloride was added dropwise to stirred dimethyl phenylphosphonite (1.5 g), while the whole system was flushed with $\mathrm{N}_{2}$ gas. The reaction mixture was stirred overnight, before lithium bromide (3.05 g) dissolved in $50 \mathrm{~mL}$ 2-butanone was added. The reaction was heated up to $50^{\circ} \mathrm{C}$ for $10 \mathrm{~min}$ and solid precipitate appeared. Then, the mixture was cooled down to room temperature and stirred for further 3 hours. The mixture was filtrated and the precipitate was washed 3 times with 2-butanone. The final product was dried in vacuum oven.<smiles>COP(OC)c1ccccc1</smiles>

Figure S3. Schematic illustration for the synthesis of LAP. 


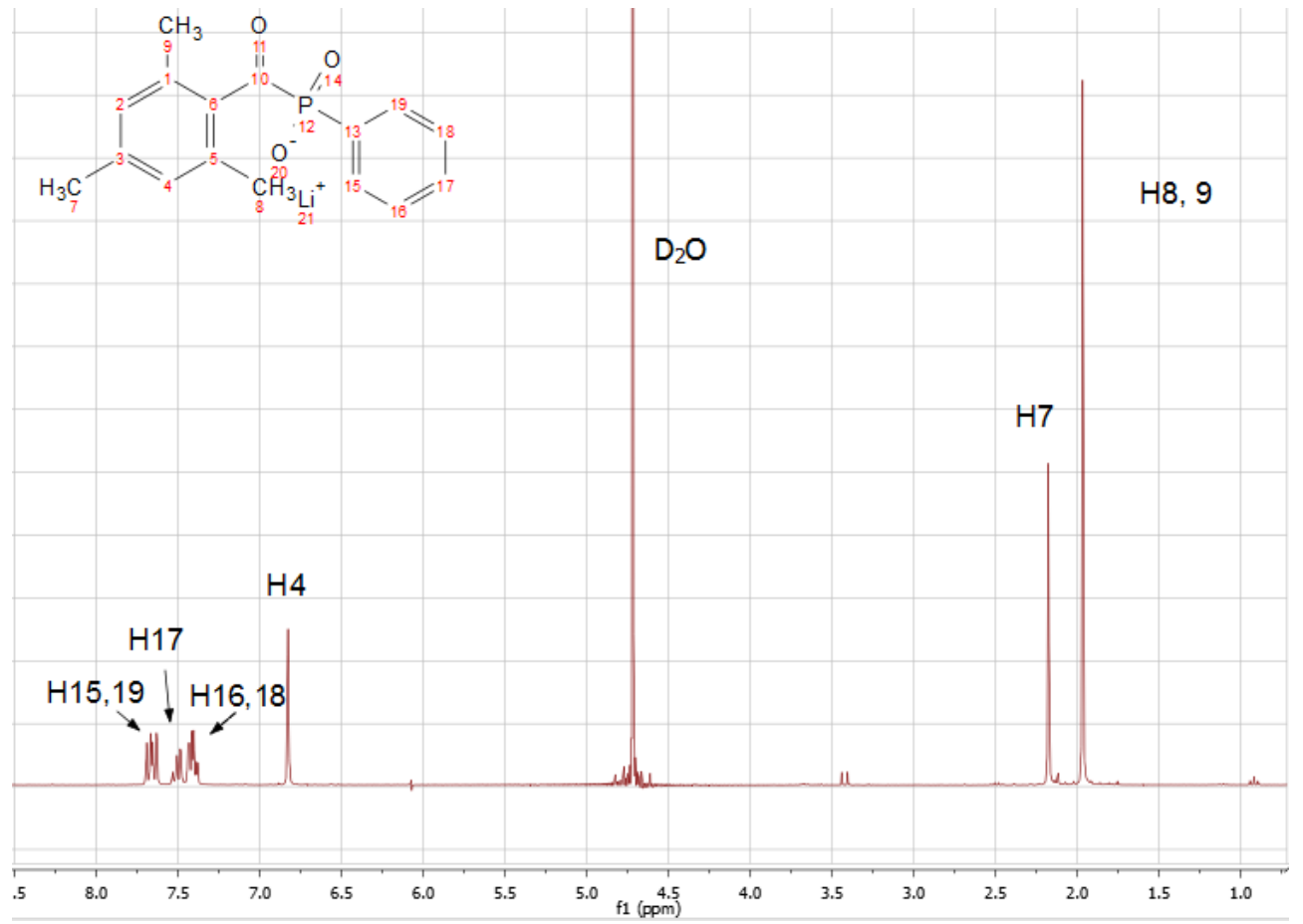

Figure S4. ${ }^{1} \mathrm{H}$ NMR spectrum of LAP in $\mathrm{D}_{2} \mathrm{O}$.

\section{Recipes of hydrogels}

Table S1. Recipes used for the preparation of hydrogels ${ }^{\mathrm{a}}$

\begin{tabular}{cccccc}
\hline & AM $(\mathrm{M})$ & PBAAM $^{\mathrm{b}}$ & DMA $^{\mathrm{b}}$ & LAP $(\mathrm{mg})$ & Volume $(\mu \mathrm{l})$ \\
\hline 1.25 & 2 & $1.25 \%$ & $1.25 \%$ & 4 & 500 \\
PBAAM-AM & 2 & $1.25 \%$ & -- & 4 & 500 \\
DMA-AM & 2 & -- & $1.25 \%$ & 4 & 500 \\
2.5 & 2 & $2.5 \%$ & $2.5 \%$ & 4 & 500 \\
5 & 2 & $5 \%$ & $5 \%$ & 4 & 500
\end{tabular}

${ }^{\mathrm{a}} \mathrm{pH}=10$, the precursors were purged with $\mathrm{N}_{2}$ gas for 5 min and further $\mathrm{UV}$-irradiated for $30 \mathrm{~min}$.

${ }^{\mathrm{b}}$ Molar ratio regarding to the amount of AM. 


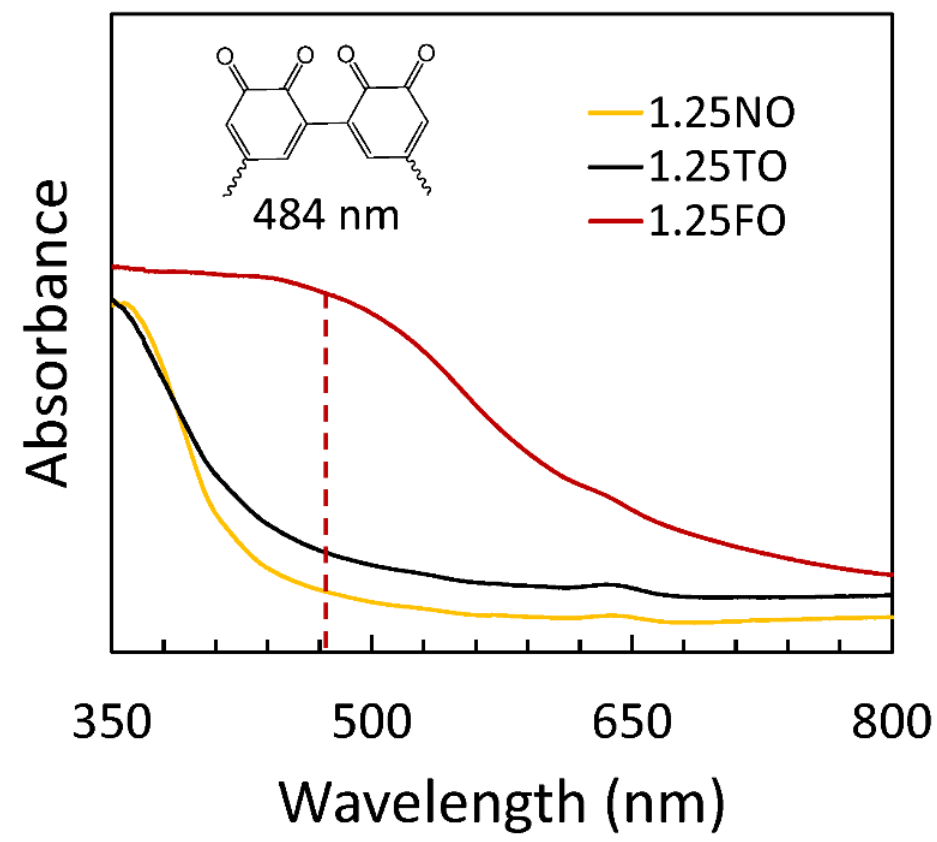

Figure S5. UV-vis spectra of 1.25NO, 1.25TO and 1.25FO. The UV-vis spectra were measured with UV-Visible Spectrophotometer Cary 300 Scan at room temperature.

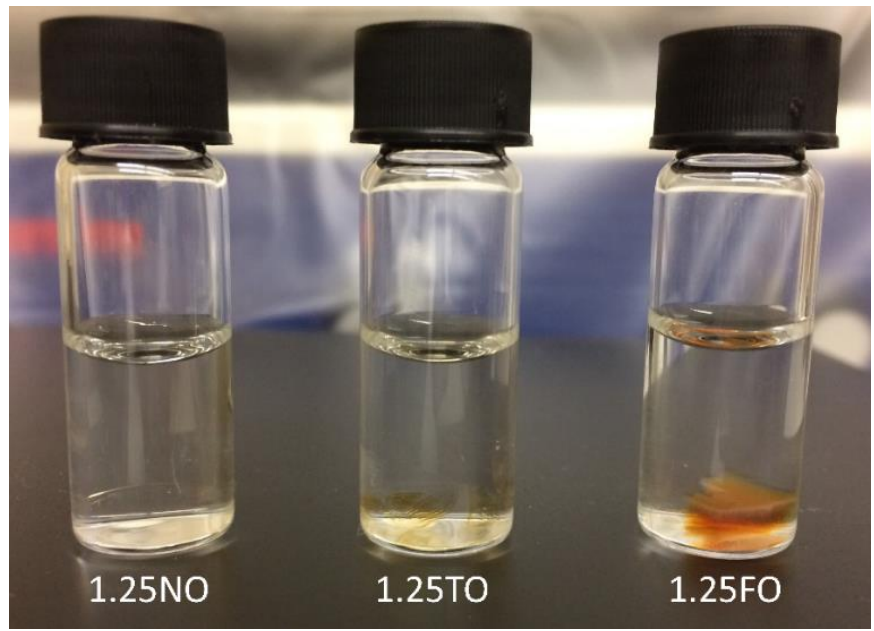

Figure S6. Photos of 1.25NO, 1.25TO and 1.25FO immersed in alkaline buffer solution ( $\mathrm{pH}=10)$ for 2 days ( $2 \mathrm{mM}$ DTT was added to avoid further oxidation). 

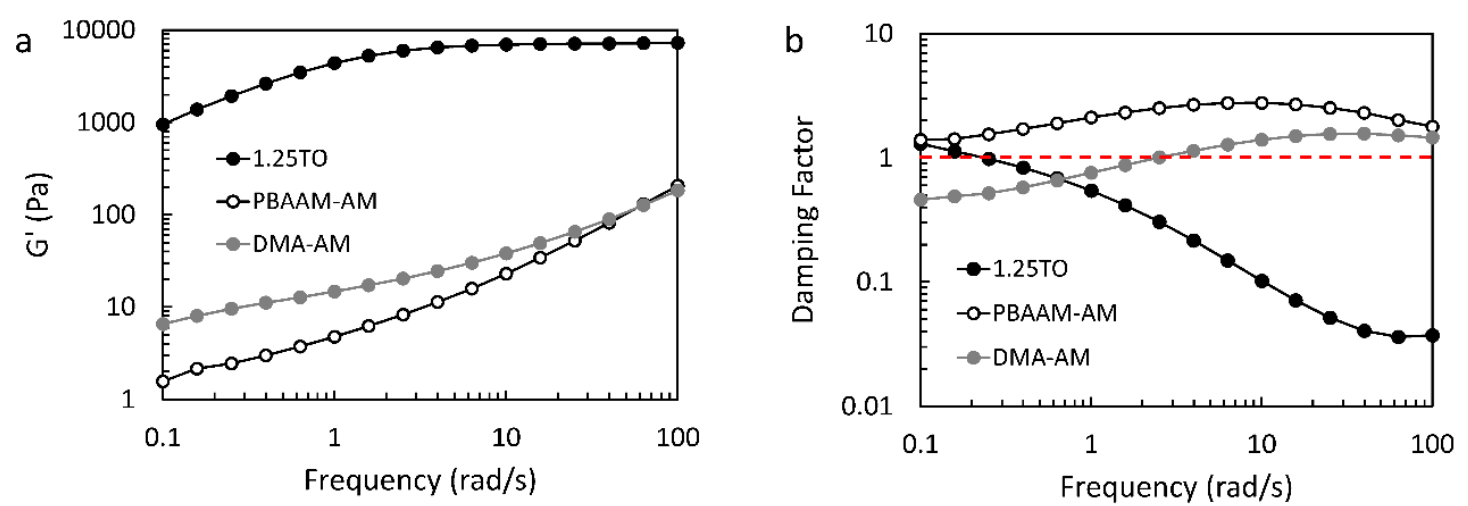

Figure S7. (a) G' and (b) damping factors of hydrogel $1.25 \mathrm{TO}$ and polymer solution PBAAM-AM and DMA-AM.

\section{Surface functionality of CNC and CNC-PBA}

The amount of the surface-exposed carboxylic groups of CNC was determined via conductivity titration. The content of carboxylic groups was evaluated using $10 \mathrm{~mL}$ of aqueous $\mathrm{CNC}$ suspension, where the $\mathrm{pH}$ value was adjusted to 2 with $0.1 \mathrm{M} \mathrm{HCl}$. The suspension solution was titrated with $0.05 \mathrm{M} \mathrm{NaOH}$ using the 800 Dosimat (Metrohm) employing a dosing rate of $0.01 \mathrm{ml} / \mathrm{s}$, while the conductivity was recorded using the 856 Conductivity Module (Metrohm) with an interval of $2 \mathrm{~s}$. 


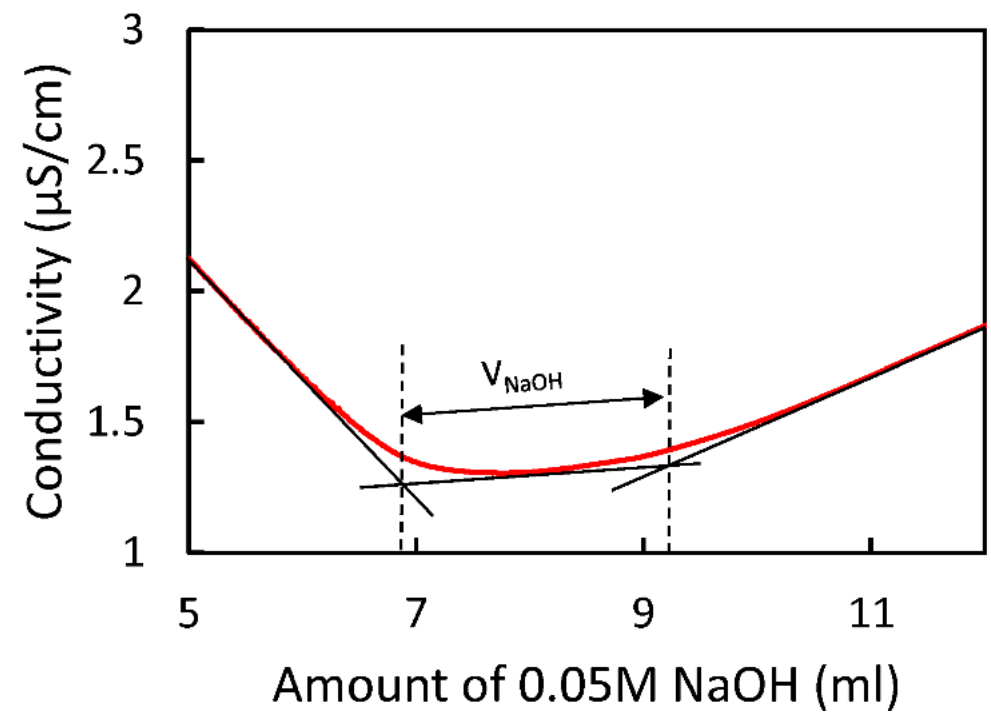

Figure S8. Conductivity titration curve of CNC.

According to the conductivity titration, the surface of virgin $\mathrm{CNC}$ was decorated with carboxyl groups of about $1.52 \pm 0.03 \mathrm{mmol} / \mathrm{g}$.

Based on the results of elemental analysis, the content of thiol groups on CNC-SH was calculated as:

$\mathrm{N}=14 * \mathrm{x}$

Where $\mathrm{N}$ was the nitrogen weight content in $\mathrm{CNC}-\mathrm{SH} ; 14$ was the molecular weight of nitrogen $(\mathrm{g} / \mathrm{mol})$; $\mathrm{x}$ indicated the molar content of cysteamine on CNC-SH $(\mathrm{mol} / \mathrm{g})$, which was the density of thiol groups on CNC-SH.

The content of PBA on CNC-PBA was calculated as:

$\mathrm{N} /(1+147.97 * \mathrm{y})=\mathrm{N}^{\prime}$

Where $\mathrm{N}$ was the nitrogen weight content in $\mathrm{CNC}-\mathrm{SH}$; N' was the nitrogen weight content in CNC-PBA; 147.97 was the molecular weight of 3-vinylphenylboronic acid (g/mol); y indicated the molar density of PBA grafted on CNC-SH ( $\mathrm{mol} / \mathrm{g})$.

Thus, the amount of thiol groups on the CNC-SH surface was determined to be $1.22 \pm 0.07$ $\mathrm{mmol} / \mathrm{g}$. The content of PBA on CNC-PBA was calculated to be $1.07 \pm 0.05 \mathrm{mmol} / \mathrm{g}$. 

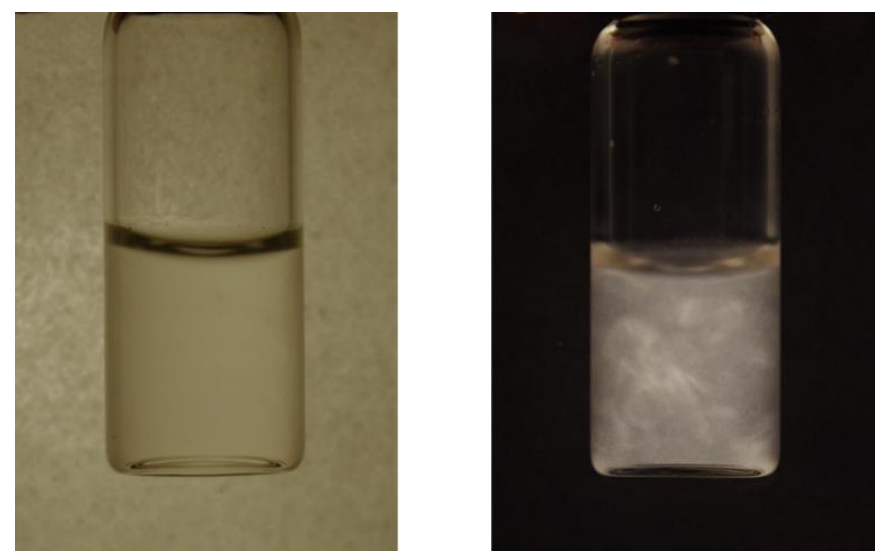

Figure S9. CNC suspension in water $(3 \mathrm{mg} / \mathrm{mL})$. Left: without polarization film; Right: between two cross polarization films.

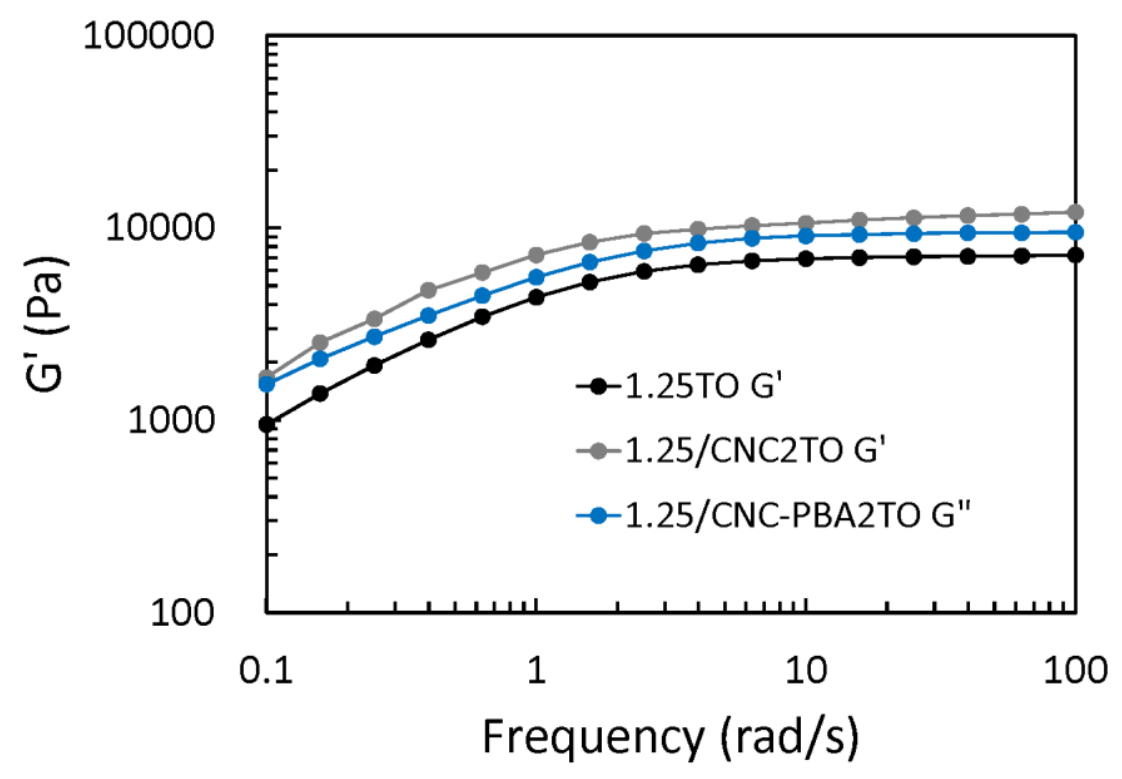

Figure S10. Rheology frequency sweeps of 1.25TO, 1.25/CNC2TO and 1.25/CNC-PBA2TO. 


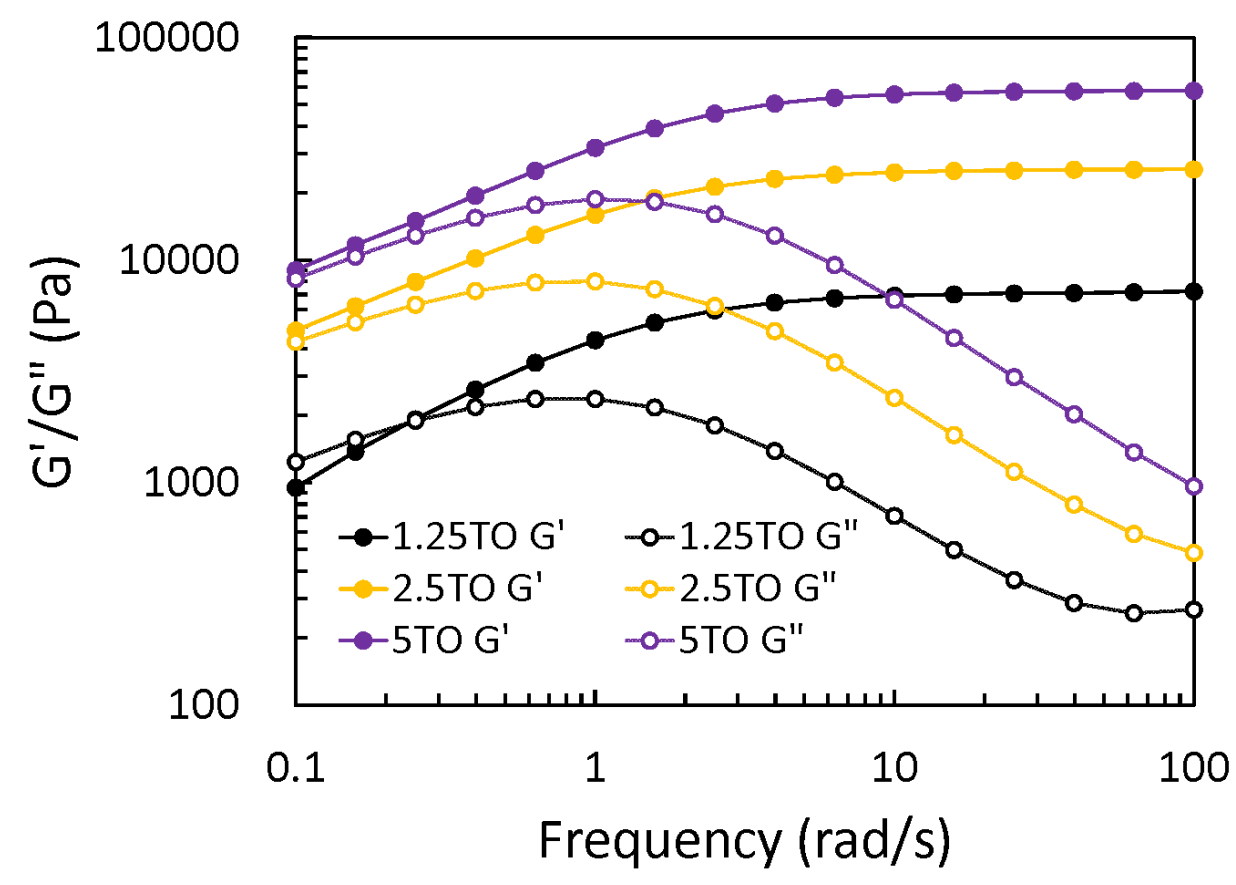

Figure S11. Rheology frequency sweeps of 1.25TO, 2.5TO and 5TO.

\section{Reference}

(1) Lee, H.; Lee, B.P.; Messersmith, P.B. Nature 2007, 448(7151), 338-341.

(2) Fairbanks, B.D.; Schwartz, M.P.; Bowman, C.N.; Anseth, K.S. Biomaterials 2009, 30(35), $6702-6707$. 


\section{Publication 3}

\section{Liquid behaviors-assisted fabrication of multidimensional birefringent materials from dynamic hybrid hydrogels}

Heqin Huang ${ }^{1}$, Xiaojie Wang ${ }^{1}$, Jinchao $\mathrm{Yu}^{2,3}$, Ye Chen ${ }^{2}$, Hong $\mathrm{Ji}^{2}$, Yumei Zhang ${ }^{2}$, Florian Rehfeldt $^{4}$, Yong Wang ${ }^{5}$ and Kai Zhang*,1

1 Wood Technology and Wood Chemistry, University of Goettingen, Büsgenweg 4, D-37077 Göttingen, Germany.

2 State Key Laboratory for Modification of Chemical Fibers and Polymer Materials, College of Materials Science and Engineering, Donghua University, Shanghai 201620, PR China 3 College of Textile and Clothing Engineering, Soochow University, Suzhou 215123, PR China

4 Third Institute of Physics - Biophysics, Faculty of Physics, University of Goettingen, Friedrich-Hund-Platz 1, D-37077 Göttingen, Germany.

5 Laboratory for Fluid Physics, Pattern Formation and Biocomplexity, Max Planck Institute for Dynamics and Self-Organization, D-37077 Göttingen, Germany.

* Tel.: +49 551 394505; E-mail: kai.zhang@uni-goettingen.de

Originally published in:

ACS Nano

American Chemical Society

DOI: 10.1021/acsnano.9b00551

Received: January 21, 2019; Revised: February 21, 2019; Published online: February 27, 2019 
Abstract: Liquid-solid transition is widely used strategy to shape polymeric materials and encode their microstructures. However, it is still challenging to fully exploit liquid behaviors of materials precursors. In particular, the dynamic and static liquid behaviors are naturally conflict to each other, which makes it difficult to integrate their advantages in the same materials. Here, by utilizing shear-thinning phenomenon in the dynamic hybrid hydrogels, we achieve a hydrodynamic alignment of cellulose nanocrystals (CNC) and preserve it in the relaxed hydrogel networks due to the much faster relaxation of polymer networks (within 500 s) than $\mathrm{CNC}$ after the unloading of external force. During the following drying process, the surface tension of hydrogels further enhances the orientation index of CNC up to 0.872 in confined geometry, these anisotropic microstructures demonstrate highly tunable birefringence (up to 0.00414 ). Due to the presence of own boundaries of dynamic hydrogels, diverse xerogels including fibers, films and even complex three-dimensional (3D) structures with variable anisotropic microstructures can be fabricated without any external molds.

Keywords: Dynamic hydrogel, cellulose nanocrystal, birefringence, anisotropy, liquid behavior

Liquid behaviors of material precursors provide infinite possibility in fabricating advanced materials. ${ }^{1}$ Well-controlled liquid precursors are powerful tools to encode anisotropic microstructures ${ }^{2}$ and generate complex shapes within materials. ${ }^{3}$ The flowing liquids have been widely used to align anisotropic particles toward the direction of shear stress, and to prepare materials with anisotropic microstructures. ${ }^{4-6}$ However, the utilization of dynamic liquid behaviors often rely on focusing fluid channels. ${ }^{7-9}$ In the static state, the liquid droplets can be governed by surface-tension to form specific shapes with minimal surface locally, including spheres, ${ }^{10}$ flat membranes ${ }^{11}$ and catenoids. ${ }^{12-13}$ Moreover, during the drying process of a static liquid system, the shrinking liquid surface confines the nonvolatile components within the liquid system and guides their assembly process. ${ }^{14-16}$ These surface tension-assisted materials fabrication is highly limited by the quiescent curing conditions, which makes it difficult to integrate liquid behaviors in dynamic and static state for material preparation. Herein, by sequentially combining their dynamic and static liquid behaviors within a liquid behaviors- 
assisted method, we achieved for the first time the tunable manipulation of anisotropic microstructures and highly variable birefringence due to organized CNC using dynamic hybrid hydrogels with own borders. By exploiting the liquid behaviors of dynamic hydrogels, ${ }^{17}$ rather than typical liquid precursors, the liquid behaviors-assisted materials fabrication can be expanded to the preparation of complex 3D structures with tunable birefringence without the dependence of external molds, such as fluid channels.

a

Dynamic hydrogels with CNC
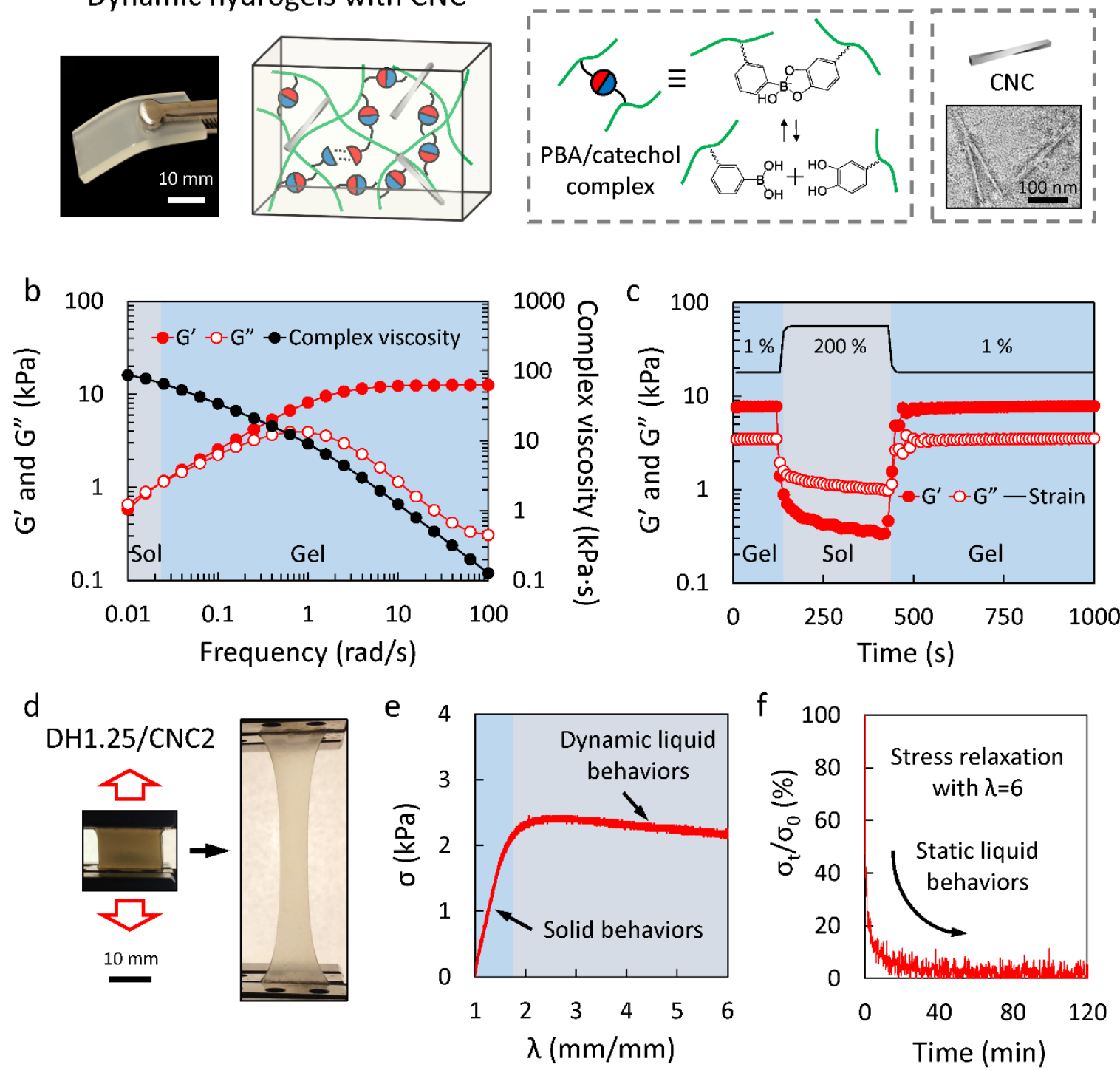

Figure 1. Dynamic hydrogels containing CNC. a) Composition of the dynamic hybrid hydrogels. b) Oscillation shear frequency sweep of DH1.25/CNC2 with constant strain 
amplitude as $1 \%$. c) The dynamic rheological 3-interval time test (3ITT) of DH1.25/CNC2. d) Uniaxial stretching of DH1.25/CNC2. e) Tensile stress-strain curve of DH1.25/CNC2. f) Tensile stress relaxation of $\mathrm{DH} 1.25 / \mathrm{CNC} 2$ with elongation ratio $\lambda=6$.

Dynamic hybrid hydrogels were prepared by in situ polymerization of acrylamide in the basic buffer with phenylboronic acid/catechol complexes (PBA/catechol complexes) as crosslinkers in the presence of CNC (Figure 1a and Figure S1). CNC, the needle-like nanowhiskers with 100-200 $\mathrm{nm}$ in length and around $10 \mathrm{~nm}$ in diameter, were obtained via 2,2,6,6tetramethylpiperidinyloxy (TEMPO)-mediated oxidation (Figure 1a and Figure S2). CNC have significant anisotropy in refractive index, which is 1.539-1.596 along the axial direction and 1.519-1.539 in the transverse direction. ${ }^{18-19}$ Moreover, CNC contained carboxyl groups of $1.50 \pm 0.02 \mathrm{mmol} / \mathrm{g}$ and aldehyde groups of $0.12 \pm 0.02 \mathrm{mmol} / \mathrm{g}$ on the surface, which allowed their good dispersion in neutral or weak basic aqueous solutions. The loading amount of CNC in the hybrid hydrogels was tuned between 0 to $2 \mathrm{wt} \%$. As inert nanofillers of low amounts, $\mathrm{CNC}$ of maximum $2 \mathrm{wt} \%$ slightly reinforced the stiffness of dynamic hydrogels, but caused little change of hydrogel relaxation behaviors, which was represented by the peak position of $\mathrm{G}^{\prime \prime}\left(\right.$ Figure S3). ${ }^{20}$

Because of the highly dynamic PBA/catechol crosslinking (binding constant $\mathrm{K}=0.919 \times 10^{3} \mathrm{M}^{-}$ $\left.{ }^{1}\right),{ }^{21}$ the mechanical behaviors of dynamic hybrid hydrogels were sensitive to the frequency of external loading (Figure 1b). With the maximal content of CNC within dynamic hydrogels, DH1.25/CNC2 (containing $2 \mathrm{~mol} / \mathrm{L}$ acrylamide with PBA/catechol complexes as $1.25 \mathrm{~mol} \%$ of acrylamide and $2 \mathrm{wt} \% \mathrm{CNC}$ ) showed a liquid state under low shear angular frequency $(<0.0251 \mathrm{rad} / \mathrm{s})$, where the storage modulus $\left(\mathrm{G}^{\prime}\right)$ exceeded the loss modulus $\left(\mathrm{G}^{\prime \prime}\right)$. Thus, in the static state, DH1.25/CNC2 showed a liquid state with high complex viscosity ( 8720 Pa.s at the angular frequency of $0.01 \mathrm{rad} / \mathrm{s}$ ). As shown in Figure S4, the sharp edges of dynamic hydrogels were slowly rounded to achieve minimal surface after 12 hours storage in sealed bottle with nitrogen gas protection.

Furthermore, another dynamic liquid state of DH1.25/CNC2 was driven by switching from low to high shearing strain. With a constant frequency of $1 \mathrm{rad} / \mathrm{s}$, the stable behaviors of static liquid 
$\left(G^{\prime}>G^{\prime \prime}\right)$ at a small shear strain of $1 \%$ were temporarily transferred to a dynamic liquid state $\left(\mathrm{G}^{\prime}<\mathrm{G}^{\prime \prime}\right)$ at a large strain of $200 \%$, as shown in Fig. 1c. Under the continuous large shearing strain, the complex viscosity was rapidly reduced from $\sim 8500 \mathrm{~Pa} \cdot \mathrm{s}$ to $\sim 2000 \mathrm{~Pa} \cdot \mathrm{s}$ within $5 \mathrm{~min}$ (Figure S5). This shear thinning property was caused by the temporary dissociation of $\mathrm{PBA} /$ catechol complexes. Once the large strain was removed, these disrupted dynamic covalent bonds were fully reconstructed within $500 \mathrm{~s}$. This reversible transition of dynamic solution-gel states with corresponding tunable viscosities was even maintained during the programming and shaping of dynamic hydrogels.

DH1.25/CNC2 exhibited elastoplastic behaviors during the uniaxial stretching (Figure 1d and Figure S6). After the elastic deformation representing the solid properties of hydrogels, dynamic hydrogels overcame a yielding point and then underwent a plastic deformation (Figure 1e and Figure S7). This plastic deformation with reduced tensile stress was generated by the shear-induced dynamic liquid properties of hydrogels. Once the stretching process was finished, the residual tensile stress of hydrogels was gradually approaching zero within $60 \mathrm{~min}$ and offset the elastic potential energy stored in polymer networks (Figure 1f), which was owing to the liquid behaviors of dynamic hydrogels in the static state.

Different to the dynamic covalent crosslinking in the molecular scale, CNC are needle-like nanostructures with the length of 100-200 $\mathrm{nm}$. After the violent shearing loading, the arrangement of $\mathrm{CNC}$ in aqueous suspension cannot be fully relaxed within $500 \mathrm{~s}$, and led to the diminished complex viscosity (Figure S8). In the stretched dynamic hydrogels, the slow relaxation of $\mathrm{CNC}$ arrangement was resisted by rapidly reconstructed hydrogel networks (Figure S5). Therefore, their footprint after the fast relaxation was preserved by the arranged $\mathrm{CNC}$, even though the dynamic liquid behaviors in the hydrogels were transient. Different from the strain-induced alignment of CNC in the elastic hydrogel matrix and thus deformed polymer matrix, ${ }^{22-23}$ the alignment of $\mathrm{CNC}$ in our dynamic hydrogels was stabilized by relaxed polymer matrix and maintained within the stretched dynamic hydrogels. 

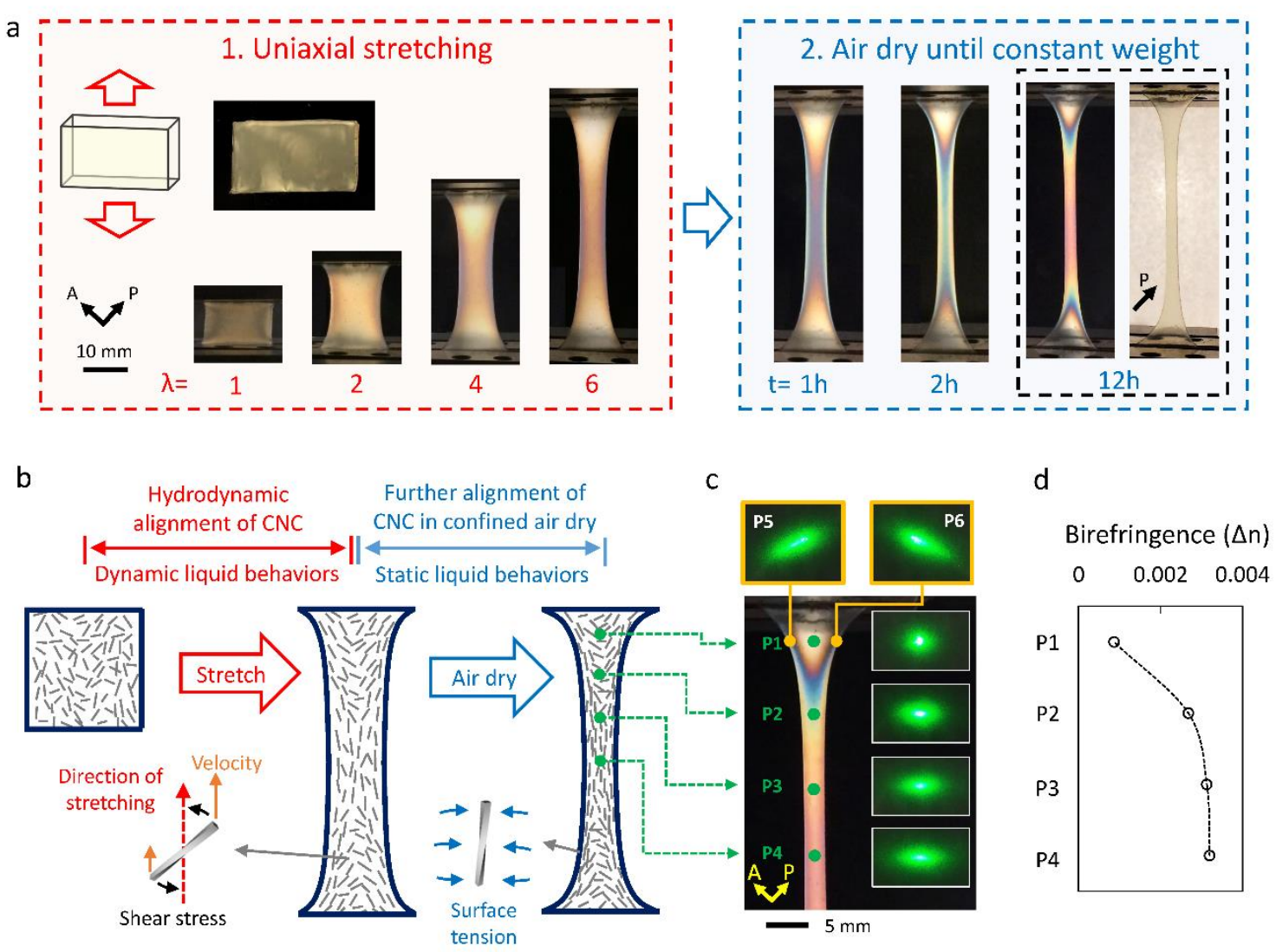

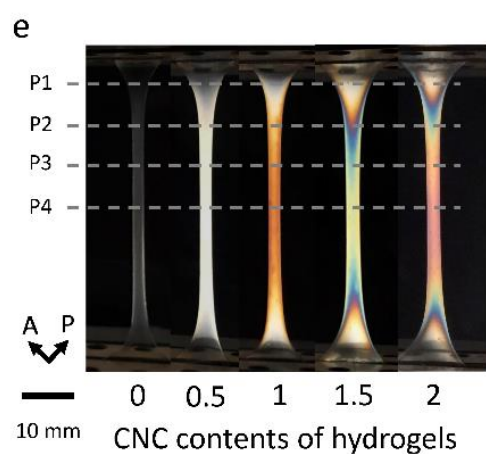

(wt\%)
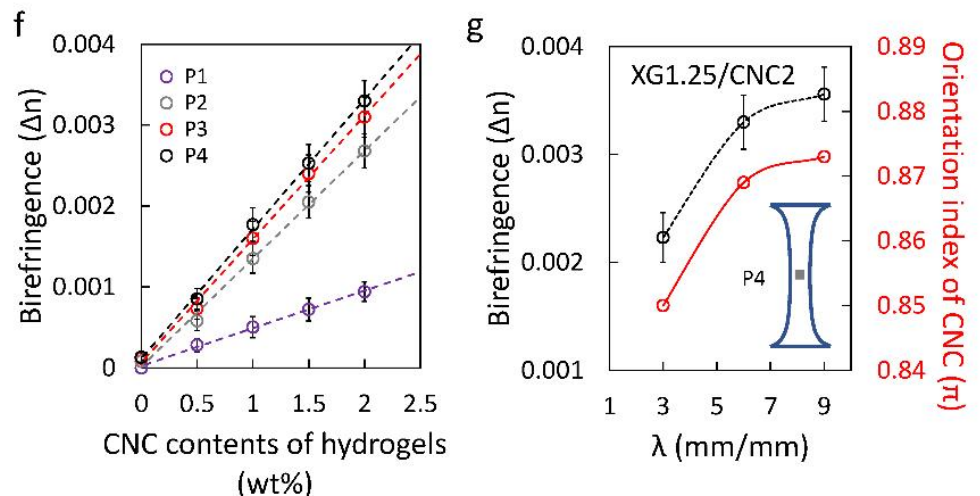

Figure 2. CNC alignment driven by the uniaxial stretching of dynamic hydrogels and following drying. a) Polarized optical images of DH1.25/CNC2 during uniaxial stretching and air dry. b) Schematic illustration for the hydrodynamic alignment of CNC in the dynamic hydrogels under stretching and their further confined assembly during the air dry. c) Laser diffraction patterns and d) corresponding birefringence of diverse regions within $\mathrm{XG1.25/CNC2}$. e, f) Polarized optical images and birefringence of XG1.25 with various CNC amounts (elongation ratio $\lambda=6)$. g) Birefringence at position No. $4(\mathrm{P} 4)$ in XG1.25/CNC2 and corresponding orientation indexes of $\operatorname{CNC}(\lambda=3,6,9)$. 
Following this, continuous interference colors were observed in the DH1.25/CNC2 during the uniaxial stretching, instead of the conventional mixed multiple colors (Figure 2a). It is well known that freely dispersed CNC can generally self-assemble into ordered cholesteric tactoid in aqueous suspensions and interfere with the light between cross polarizers (Figure S2). ${ }^{24-25}$ These noncontinuous liquid crystal structures were formed before the in situ polymerization without external interference and were stabilized in the dynamic hydrogels networks. In comparison, the presence of continuous colors in our stretched dynamic hydrogels represents an altered assembly state of CNC during the uniaxial stretching. Moreover, the dynamic hydrogel DH1.25 without CNC, showed no interference colors under otherwise equal conditions (Figure S9). In addition, the biaxial stretching offset the interference colors in DH1.25/CNC2 (Figure S10). Thus, the uniaxial stretching rearranged the assembled CNC within the hydrogels due to different relaxation times of hydrogel networks and CNC, leading to continuous interference colors.

These continuous interference colors were further enhanced by drying the stretched dynamic hydrogels in air, where the static liquid behaviors and the surface tension primarily affect the rearrangement of hydrogel networks. After drying at a constant elongation ratio $\lambda=6$, xerogel XG1.25/CNC2 demonstrated further amplified rainbow-like interference colors with continuous color distributions. These interference colors reached the maximum intensity, when the xerogels were observed between cross polarizers with the stretching direction of 45 degrees against the polarizers. In comparison, nearly no interference signal was observed, when the stretching direction was parallel or perpendicular to the polarizers (Figure S11). Therefore, originally cholesteric phases of $\mathrm{CNC}$ in hydrogels became a nematic phase along the stretching direction, and amplified the anisotropy of individual CNC to the macrosized dried xerogels.

Figure $2 \mathrm{~b}$ schematically illustrates the $\mathrm{CNC}$ alignment during the sequential stretching process and followed air dry. During the stretching, the dynamic hydrogels were deformed with positive Poisson's ratio and the whole dynamic hydrogels became contracted. Because two edges were fixed during the stretching, the contraction became greater from both fixed edges to the middle of the hydrogels. Moreover, long-distance stretching reduced local complex 
viscosity because of the fast dissociation of PBA/catechol complexes, and drove a hydrodynamic alignment of $\mathrm{CNC}$ within the confined hydrogels. Once the stretching process was terminated, the high viscosity of hydrogels recovered due to the fast reconstruction of $\mathrm{PBA} /$ catechol complexes as crosslinkers and fixed the rearranged CNC.

Subsequent air dry of stretched dynamic hydrogels caused further lateral volume contraction and the largest shear stress gradient in the regions close to the edges, ${ }^{26}$ which further enhanced the organization of CNC. This further enhanced orientation of CNC was approved by laser scattering patterns of diverse regions within XG1.25/CNC2 (positions 1-6, P1-P6) (Figure 2c and Figure S12). P1-P4 (with interval distance of $8 \mathrm{~mm}$ ) exhibited the gradually improved anisotropy from P1 to P4 along the contraction of xerogels. Compared with P1 at the centerline, P5 and P6 at the curved edges of the XG1.25/CNC2 with the same altitude showed much larger anisotropy. The P1 demonstrates a region within the interior of the original hydrogels and was less affected than the edges. In comparison, the P4 represents a region where two edges merge into each other during air dry and thus most largely enhanced the orientation of CNC.

Furthermore, these interference colors observed between cross polarizers are because of light retardations, which are accumulated phase differences between lights vibrating perpendicular and parallel to aligned $\mathrm{CNC} .{ }^{27}$ The light retardation can be described by using the birefringence, which is generally quantified as the maximum difference between refractive indexes exhibited by anisotropic materials. In the XG1.25/CNC2, the birefringence increased accordingly from $0.00094 \pm 0.00012$ (P1) to $0.0033 \pm 0.0003$ (P4, center of specimen) in XG.25/CNC2 (Figure 2d), which quantitatively showed the improved alignment of CNC.

Although polyacrylamide networks were also slightly aligned during the confined drying process, it contributed only slightly $(<5 \%)$ to the formation of interference colors in $\mathrm{XG1.25/CNC2}$ (Figure 2e). Almost no significant color is observable, if no CNC is present within the xerogel. In comparison, the orders of continuous interference colors increased with higher $\mathrm{CNC}$ amounts within xerogels from $0.5 \mathrm{wt} \%$ to $2 \mathrm{wt} \%$. At the same time, the birefringence of xerogels at the same positions (P1-P4) was positively improved with higher 
dosage of CNC (Figure 2f), which further confirmed the alignment of $\mathrm{CNC}$ as the main source for the birefringence in the xerogels.

Moreover, larger elongations of dynamic hydrogels generated further contraction that promoted the hydrodynamic alignment of CNC. As shown on XG1.25/CNC2 (Fig. 2g), the birefringence of xerogels was improved from 0.00223 to 0.00356 with the elongation ratios from 3 to 9. Importantly, with the same dosage of $\mathrm{CNC}$, the birefringence of xerogels improved positively along with the increase of $\mathrm{CNC}$ orientation indexes (from 0.850 to 0.873 ) (Figure $2 \mathrm{~g}$ and Figure S13). Therefore, the orientation of CNC can transfer the birefringence of CNC in nanoscale to the xerogels of macroscale. Furthermore, the crosslinking density of hydrogels simultaneously affected the Poisson's ratio of and shear stress in the dynamic hydrogels, but they led to similar maximum birefringence in the resulted xerogels (Figure S14). Therefore, the organization of $\mathrm{CNC}$ in addition to the amount of $\mathrm{CNC}$ in the light pathway predominantly determines the retardation of light and thus the interference colors of xerogels. 


\section{a}

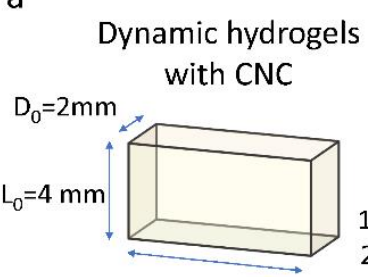

$\mathrm{W}_{0}$

$$
\text { b }
$$

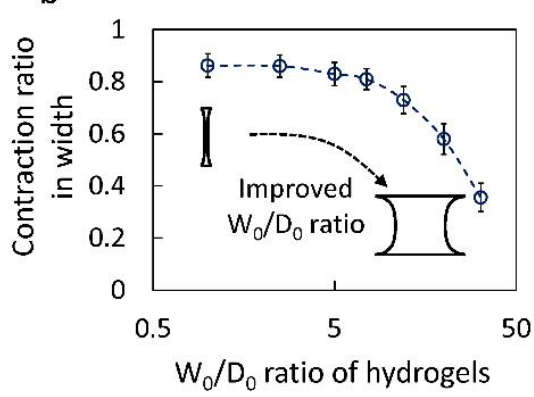

$\mathrm{C}$

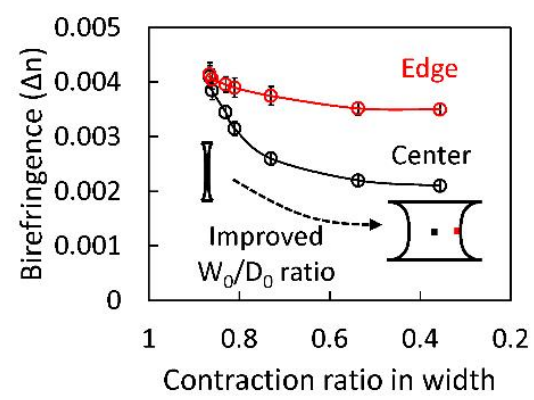

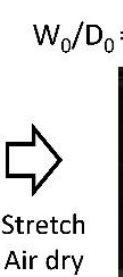

Ap $-10 \mathrm{~mm}$
20

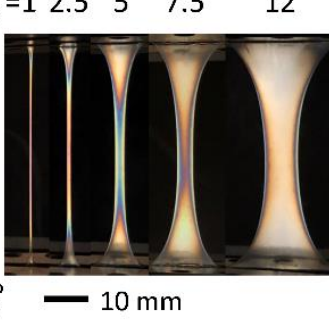

32

d

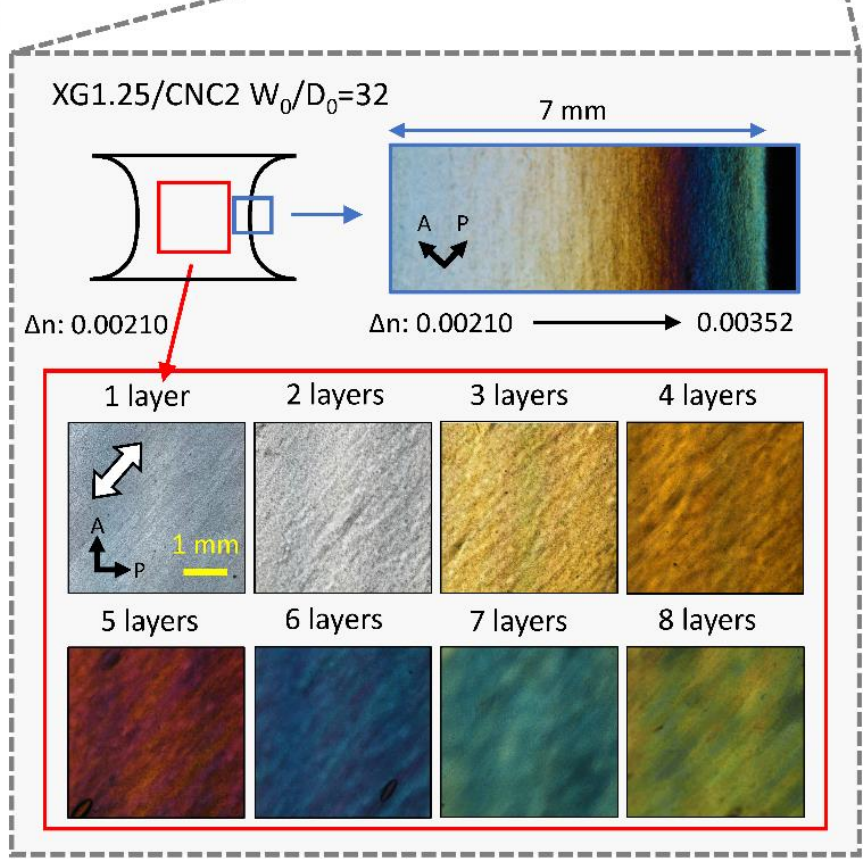

Figure 3. Geometry of dynamic hydrogels regulating the alignment of CNC in xerogels.

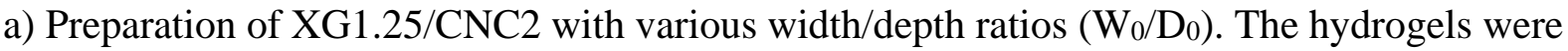
stretched with the same elongation ratio $\lambda=10$. b) Contraction ratio in width of xerogels with various $\mathrm{W}_{0} / \mathrm{D}_{0}$ ratios. c) Birefringence of xerogels at $\mathrm{P} 4$ middle (black point) and $\mathrm{P} 4$ edge (red point). d) Distribution of gradient birefringence in the edge area and uniform birefringence in the center area of $\mathrm{XG1.25/CNC2}$ with the original $\mathrm{W}_{0} / \mathrm{D}_{0}=32$. The white arrow indicates the direction of stretching.

In addition to the birefringence, the aligned $\mathrm{CNC}$ in the xerogels also lead to anisotropic mechanical properties (Figure S18). The film is not only stiffer, but also tougher in the direction of $\mathrm{CNC}$ alignment. In the central area of $\mathrm{XG1.25/CNC2}$ with the original $\mathrm{W}_{0} / \mathrm{D}_{0}=32$, the fracture energy along $\mathrm{CNC}$ alignment was up to $81.6 \pm 8.8 \mathrm{~kJ} / \mathrm{m}^{2}$, while the fracture energy 
vertical to CNC alignment was only $34.1 \pm 4.2 \mathrm{~kJ} / \mathrm{m}^{2}$. The drawing in the direction of CNC alignment caused ductile fracture with a white damage zone, but not significant if drawing in the perpendicular direction (Figures $3 \mathrm{f}-\mathrm{g}$ ). The white damage zone was caused by the formation of wrinkled structure, which dissipated energy and performed a plastic deformation before the rupture (Figure S19).

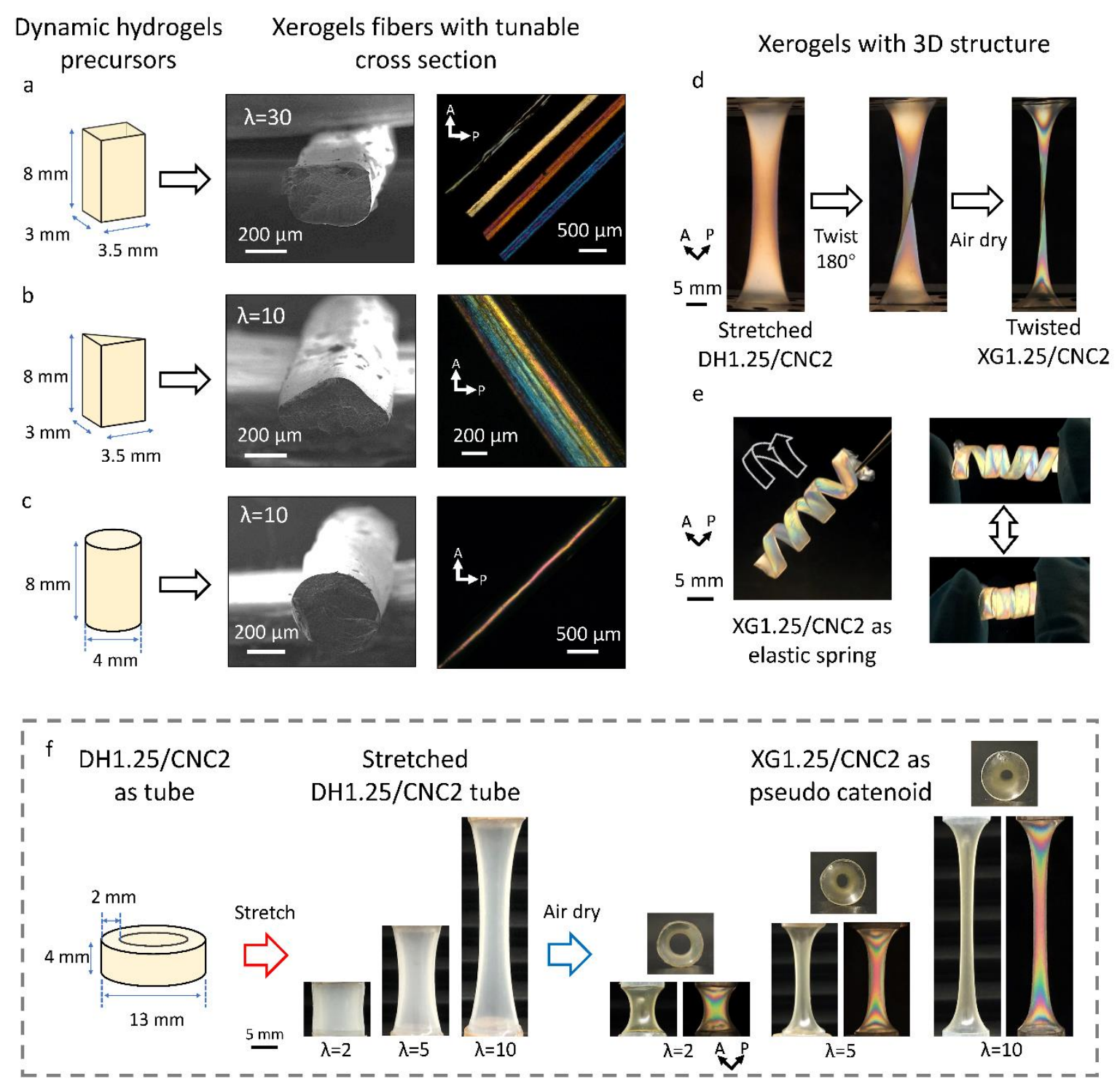

Figure 4. Dynamic hydrogels and xerogels with programmable shapes. a) XG1.25/CNCX fibers with rectangular cross-section. The dark field polarized optical images from left top to right bottom: $\mathrm{CNC}$ amounts $\mathrm{x}$ were $0.5,1,1.5$ and $2 \mathrm{wt} \%$ in the original dynamic hydrogels. b) $\mathrm{XG} 1.25 / \mathrm{CNC} 2$ fibers with triangular cross-section. c) $\mathrm{XG} 1.25 / \mathrm{CNC} 2$ fibers with round 
cross-section. d) XG1.25/CNC2 with twisted structure (after the stretching with the elongation ratio $\lambda=6$ ). e) $\mathrm{XG1} .25 / \mathrm{CNC} 2$ as elastic spring. f) $\mathrm{XG} 1.25 / \mathrm{CNC} 2$ as pseudo catenoid after stretching initial dynamic hydrogels in the tube form with various elongation ratios $\lambda=2,5$ and 10.

The shape of resulted xerogels can be encoded with adjustable shapes because of the static liquid properties of dynamic hydrogels. In Figures 4a-c, the xerogel fibers can be prepared with rectangular, triangular or round cross-section after the stretching with the elongation ratios of 10-30. The fibers with rectangular and round cross-section exhibited uniform interference colors. For the fibers with triangular cross-section, the hypotenuse of triangle led to gradual depth of fibers and showed gradient interference colors. In particular, the colors within fibers could be tuned by changing the content of $\mathrm{CNC}$ during the preparation of hydrogel precursors (Figure 4a). It is notable that greater elongation was beneficial in preserving the shape of hydrogels (Figure S20). This is because the thinner fibers dried faster and resisted stronger the shape change by surface tension in the static liquid state.

In addition, the sequential controllability of static and dynamic properties of dynamic hydrogels provide the flexibility to program spatial complex 3D shapes with well-ordered $\mathrm{CNC}$, which is generally difficult for flow-assisted methods in classic liquid systems. For instance, with the help of interfacial jamming of nanoparticle surfactants, ${ }^{28-29}$ liquid droplets can be stabilized in nonequilibrium shape, but the liquid enclosed by this robust interface is fully relaxed. By using dynamic hydrogels in the present work, the shapes of stretched hydrogels can be reprogramed before the drying process, while CNC still can be adjustably aligned and provide tunable photonic properties. As examples, twisted xerogels were prepared by rotating stretched DH1.25/CNC2 axially before the drying process (Figure 4d). By twining around a Teflon stick, stretched DH1.25/CNC2 can be turned into an elastic xerogel spring with interference colors (Figure 4e).

Hollow dynamic hydrogels as a further model for complex 3D shapes were prepared (Figure 4f). The tubular xerogels were obtained from dynamic hydrogel tubes after following air dry. DH1.25/CNC2 tube was comparatively equivalent to the rolled-up film of DH1.25/CNC2 with 
$\mathrm{W}_{0} / \mathrm{D}_{0}=20$ with the edges merged together. The tube shape and the absence of edges allow on the one side the uniform organization of $\mathrm{CNC}$ at the same altitude and on the other side equivalent observation results (the same interference colors independent on the observation angles). During the drying process, the circular tube wall of hydrogels underwent a homogeneous contraction within the hydrogel walls and thus $\mathrm{CNC}$ at the same altitude were uniformly aligned along the stretching direction. Because of the homogenized CNC alignment, the maximum birefringence at the center of $\mathrm{XG1.25/ \textrm {CNC } 2}$ tube was measured as $0.00283 \pm 0.00025$, which was lower than that $(0.00352 \pm 0.00012)$ in the central area of $\mathrm{XG1.25/CNC2}$ membranes with original $\mathrm{W}_{0} / \mathrm{D}_{0}=20$, but higher than their edge areas $(0.00220 \pm 0.00010)$ (Figure S21).

In contrast to hydrogel columns with solid interior, dried xerogel tubes developed concave structures towards the center of tubes, which was driven by surface tension of hydrogels to pursue the minimal surface minimal surface (Figure 4f, the insets of the pseudo catenoid). Following this, the hollow xerogels demonstrate a pseudo catenoid-mimetic morphology, which can be further regulated by the elongation ratio of dynamic hybrid hydrogels.

\section{CONCLUSION}

In conclusion, we demonstrated that the liquid behaviors-assisted material fabrication can be expanded to 3D morphology without the limitation of fluid channels, which was owing to the dynamic hybrid hydrogels with both solid properties and liquid properties. By stretching this dynamic hydrogel with $\mathrm{CNC}$, we sequentially integrated dynamic and static liquid behaviors to program diverse polymeric materials. After the uniaxial stretching, a transient liquid state was created and consequently drove the alignment of anisotropic $\mathrm{CNC}$ in the dynamic hydrogels. Importantly, their organization was preserved, even when the temporary dynamic liquid state of hydrogels matrix had dissipated. The stretched dynamic hydrogels were then dried in air and transformed into xerogels to further improve the aligned CNC and generated highly tunable birefringence. The shape of resultant xerogels can be versatile via remolding before the drying process, e.g. in the shapes of fibers, twists, springs and pseudo catenoid with concave surface. Therefore, the dynamic hydrogels containing anisotropic nanoparticles, 
provide a new strategy as a mold-free methodology to program dry polymers with various 3D shapes and anisotropic microstructures.

\section{METHODS}

Preparation of CNC. CNC was prepared with TEMPO-mediated oxidation of MCC according to the previous report. ${ }^{30}$ To remove large particles after the synthesis, the purified CNC (about $6 \mathrm{mg} / \mathrm{mL}$ ) was stored at room temperature for 24 hours. Then the precipitated large particles were removed, and the upper stable suspension was collected and stored in the fridge for further use.

Preparation of dynamic hybrid hydrogels. Dynamic hybrid hydrogels in the present work were prepared via UV initiated radical polymerization between glass slides. Polyvinyl chloride films were used as spacer to control the hydrogel thickness. The alkaline aqueous solution was prepared with borax- $\mathrm{NaOH}$ buffer with $\mathrm{pH}$ of 10. LAP was used as photoinitiator with $0.5 \mathrm{wt} \%$ of the whole hydrogels. The precursors were purged with $\mathrm{N}_{2}$ gas or argon for 5 min to remove dissolved oxygen. Then precursors were exposed to the UV light (wavelength from $300 \mathrm{~nm}$ to $400 \mathrm{~nm}$ ) for $30 \mathrm{~min}$ to finish the gelation process.

Preparation of xerogels. The uniaxial stretching of dynamic hybrid hydrogels were performed with a Z3 micro tensile test machine from Grip-Engineering Thümler $\mathrm{GmbH}$ or dynamic thermal mechanical analysis machine EPLEXOR® $500 \mathrm{~N}$ from Gabo/Netzsch. The tensile tests were performed at $\sim 20{ }^{\circ} \mathrm{C}$ and a relative humidity of $65 \%$. The default tensile tests speed was $2.5 \mathrm{~mm} / \mathrm{min}$. The default dimension of hydrogels for tensile tests is $15 \mathrm{~mm}$ in width, $2 \mathrm{~mm}$ in thickness and length in 4 or $8 \mathrm{~mm}$. The default dimension of xerogel films for tensile tests is $7-8 \mathrm{~mm}$ in width and $5 \mathrm{~mm}$ in length. The following air drying process was preformed at $20^{\circ} \mathrm{C}$ and a relative humidity of $65 \%$ until constant weight.

\section{ASSOCIATED CONTENT}

\section{Supporting Information}


Supporting Figures S1-S21, experimental details, characterization of dynamic hybrid hydrogels and xerogels (PDF).

\section{AUTHOR INFORMATION}

\section{Corresponding Authors}

*E-mail: kai.zhang@uni-goettingen.de

\section{ACKNOWLEDGEMENTS}

K.Z. thanks the German Research Foundation (DFG) for the project ZH546/3-1 for the financial support. The program "Material Science Wood" from Lower Saxony Ministry of Science and Culture, Germany, is gratefully acknowledged for the financial support. X.W. thanks the China Scholarship Council (CSC) for financial support. The authors also thank Ms. Huan Liu for assistance with scanning electron microscope and laser scanning microscope, and thank Dr. Jianguo Zhao and Dr. Hongyan Jiang for helpful discussion.

\section{REFERENCES}

(1) Asthana, R.; Kumar, A.; Dahotre, N., B., Materials Processing and Manufacturing Science. Elsevier: 2006.

(2). Kamadaa, A.; Mittal, N.; Söderberg, L. D.; Ingverud, T.; Ohmd, W.; Roth, S. V.; Lundell, F.; Lendele, C., Flow-assisted assembly of nanostructured protein microfibers. Proc. Natl. Acad. Sci. U. S. A. 2017, 114 (6), 1232-1237.

(3). Rofouie, P.; Alizadehgiashi, M.; Mundoor, H.; Smalyukh, I. I.; Kumacheva, E., SelfAssembly of Cellulose Nanocrystals into Semi-Spherical Photonic Cholesteric Films. Adv. Funct. Mater. 2018, 28 (45), 1803852.

(4). Hakansson, K. M.; Fall, A. B.; Lundell, F.; Yu, S.; Krywka, C.; Roth, S. V.; Santoro, G.; Kvick, M.; Prahl Wittberg, L.; Wagberg, L.; Soderberg, L. D., Hydrodynamic alignment and assembly of nanofibrils resulting in strong cellulose filaments. Nat. Commun. 2014, 5, 4018.

(5) Mittal, N.; Ansari, F.; Gowda, V. K.; Brouzet, C.; Chen, P.; Larsson, P. T.; Roth, S. V.; Lundell, F.; Wagberg, L.; Kotov, N. A.; Soderberg, L. D., Multiscale Control of Nanocellulose 
Assembly: Transferring Remarkable Nanoscale Fibril Mechanics to Macroscale Fibers. ACS nano 2018, 12 (7), 6378-6388.

(6) Ling, S.; Qin, Z.; Li, C.; Huang, W.; Kaplan, D. L.; Buehler, M. J., Polymorphic regenerated silk fibers assembled through bioinspired spinning. Nat. Commun. 2017, 8 (1), 1387.

(7) Gantenbein, S.; Masania, K.; Woigk, W.; Sesseg, J. P. W.; Tervoort, T. A.; Studart, A. R., Three-dimensional printing of hierarchical liquid-crystal-polymer structures. Nature 2018, 561 (7722), 226-230.

(8) Kim, Y.; Yuk, H.; Zhao, R.; Chester, S. A.; Zhao, X., Printing ferromagnetic domains for untethered fast-transforming soft materials. Nature 2018, 558 (7709), 274-279.

(9) Hausmann, M. K.; Ruhs, P. A.; Siqueira, G.; Lauger, J.; Libanori, R.; Zimmermann, T.; Studart, A. R., Dynamics of Cellulose Nanocrystal Alignment during 3D Printing. ACS nano 2018, 12 (7), 6926-6937.

(10)Zhao, Y.; Shang, L.; Cheng, Y.; Gu, Z., Spherical colloidal photonic crystals. Acc. Chem. Res. 2014, 47 (12), 3632-3642.

(11)Ragelle, H.; Tibbitt, M. W.; Wu, S. Y.; Castillo, M. A.; Cheng, G. Z.; Gangadharan, S. P.; Anderson, D. G.; Cima, M. J.; Langer, R., Surface tension-assisted additive manufacturing. Nat. Commun. 2018, 9 (1), 1184.

(12)Wang, L.; McCarthy, T. J., Capillary-bridge-derived particles with negative Gaussian curvature. Proc. Natl. Acad. Sci. U. S. A. 2015, 112 (9), 2664-2669.

(13)Kim, E.; Witesides, G. M., Use of Minimal Free Energy and Self-Assembly To Form Shapes. Chem. Mater. 1995, 7 (6), 1257-1264.

(14)Tseng, P.; Napier, B.; Zhao, S.; Mitropoulos, A. N.; Applegate, M. B.; Marelli, B.; Kaplan, D. L.; Omenetto, F. G., Directed assembly of bio-inspired hierarchical materials with controlled nanofibrillar architectures. Nat. Nanotechnol. 2017, 12 (5), 474-480.

(15)Parker, R. M.; Frka-Petesic, B.; Guidetti, G.; Kamita, G.; Consani, G.; Abell, C.; Vignolini, S., Hierarchical Self-Assembly of Cellulose Nanocrystals in a Confined Geometry. ACS nano 2016, 10 (9), 8443-8449. 
(16)Yao, K.; Meng, Q.; Bulone, V.; Zhou, Q., Flexible and Responsive Chiral Nematic

Cellulose Nanocrystal/Poly(ethylene glycol) Composite Films with Uniform and Tunable Structural Color. Adv. Mater. 2017, 29 (28), 1701323.

(17)Zhang, Y. S.; Khademhosseini, A., Advances in engineering hydrogels. Science 2017, 356 (6337), eaaf3627.

(18)Nogi, M.; Yano, H., Transparent Nanocomposites Based on Cellulose Produced by Bacteria Offer Potential Innovation in the Electronics Device Industry. Adv. Mater. 2008, 20 (10), 1849-1852.

(19) Yano, H.; Sugiyama, J.; Nakagaito, A. N.; Nogi, M.; Matsuura, T.; Hikita, M.; Handa, K., Optically Transparent Composites Reinforced with Networks of Bacterial Nanofibers. Adv. Mater. 2005, 17 (2), 153-155.

(20)Grindy, S. C.; Learsch, R.; Mozhdehi, D.; Cheng, J.; Barrett, D. G.; Guan, Z.; Messersmith, P. B.; Holten-Andersen, N., Control of hierarchical polymer mechanics with bioinspired metalcoordination dynamics. Nat. Mater. 2015, 14 (12), 1210-1216.

(21)Zhao, P.; Wei, K.; Feng, Q.; Chen, H.; Wong, D. S. H.; Chen, X.; Wu, C. C.; Bian, L., Mussel-mimetic hydrogels with defined cross-linkers achieved via controlled catechol dimerization exhibiting tough adhesion for wet biological tissues. Chem. Commun. 2017, 53 (88), 12000-12003.

(22)Mredha, M. T. I.; Guo, Y. Z.; Nonoyama, T.; Nakajima, T.; Kurokawa, T.; Gong, J. P., A Facile Method to Fabricate Anisotropic Hydrogels with Perfectly Aligned Hierarchical Fibrous Structures. Adv. Mater. 2018, 30 (9), 1704937.

(23)Osorio-Madrazo, A.; Eder, M.; Rueggeberg, M.; Pandey, J. K.; Harrington, M. J.; Nishiyama, Y.; Putaux, J. L.; Rochas, C.; Burgert, I., Reorientation of cellulose nanowhiskers in agarose hydrogels under tensile loading. Biomacromolecules 2012, 13 (3), 850-856.

(24)Wang, P. X.; Hamad, W. Y.; MacLachlan, M. J., Structure and transformation of tactoids in cellulose nanocrystal suspensions. Nat. Commun. 2016, 7, 11515.

(25)Parker, R. M.; Guidetti, G.; Williams, C. A.; Zhao, T.; Narkevicius, A.; Vignolini, S.; FrkaPetesic, B., The Self-Assembly of Cellulose Nanocrystals: Hierarchical Design of Visual Appearance. Adv. Mater. 2018, 30 (19), e1704477. 
(26)Kim, J.; Michelin, S.; Hilbers, M.; Martinelli, L.; Chaudan, E.; Amselem, G.; Fradet, E.; Boilot, J. P.; Brouwer, A. M.; Baroud, C. N.; Peretti, J.; Gacoin, T., Monitoring the orientation of rare-earth-doped nanorods for flow shear tomography. Nat. Nanotechnol. 2017, 12 (9), 914919.

(27)Zhang, G.; Peng, W.; Wu, J.; Zhao, Q.; Xie, T., Digital coding of mechanical stress in a dynamic covalent shape memory polymer network. Nat. Commun. 2018, 9 (1), 4002.

(28)Cui, M.; Emrick, T.; Russell, T. P., Stabilizing Liquid Drops in Nonequilibrium Shapes by the Interfacial Jamming of Nanoparticles. Science 2013, 342 (6157), 460-463.

(29)Shi, S.; Liu, X.; Li, Y.; Wu, X.; Wang, D.; Forth, J.; Russell, T. P., Liquid Letters. Adv. Mater. 2018, 30 (9), 1705800.

(30)Liu, J.; Plog, A.; Groszewicz, P.; Zhao, L.; Xu, Y.; Breitzke, H.; Stark, A.; Hoffmann, R.; Gutmann, T.; Zhang, K.; Buntkowsky, G., Design of a Heterogeneous Catalyst Based on Cellulose Nanocrystals for Cyclopropanation: Synthesis and Solid-State NMR Characterization. Chem. Eur. J. 2015, 21, 12414. 


\section{Publication 3 (Supporting information)}

\section{Liquid behaviors-assisted fabrication of multidimensional birefringent materials from dynamic hybrid hydrogels}

Heqin Huang, Xiaojie Wang, Jinchao Yu, Ye Chen, Hong Ji, Yumei Zhang, Florian Rehfeldt, Yong Wang and Kai Zhang*

\section{Materials}

Microcrystalline cellulose (MCC), 2,2,6,6-tetramethylpiperidine 1-oxyl (TEMPO), 3(acrylamido)phenylboronic acid (PBAAM), methacrylic anhydride, sodium hydrochloride, phosphotungstic acid hydrate, 2,4,6-trimethylbenzoyl chloride were purchased from SigmaAldrich (USA). Dopamine hydrochloride, dimethyl phenylphosphonite, and 2-butanone were obtained from Alfa Aesar (USA). Acrylamide (AM), sodium sulfate anhydrous, disodium tetraborate were bought from Merck Millipore (USA). Sodium hydroxide was ordered from VWR (Germany). Sodium bicarbonate was bought from TH Geyer (Germany). Hydrochloric acid (37\%) was obtained from AppliChem (Germany). The organic solutions, including tetrahydrofuran (THF), ethyl acetate, nhexane, and acetone, were all purchased from TH Geyer (Germany). Deionized water utilized throughout all experiments was purified from a Millipore system. Dopamine methylacrylate ${ }^{1}$ and lithium phenyl-2,4,6-trimethylbenzoylphosphinate $(\mathrm{LAP})^{2}$ were synthesized with previous reported method.

\section{Characterization}

The rheological properties of hydrogels were investigated with a stress-controlled bulk rheometer (Anton Paar MCR501) by using a $25 \mathrm{~mm}$ parallel-plate setup. In each test, $1 \mathrm{~mL}$ of hydrogel of each sample was prepared in advance. Storage modulus (G'), loss modulus (G’), damping factor and complex viscosity were recorded. All of the rheology measurements were carried out at room temperature $\left(\sim 23{ }^{\circ} \mathrm{C}\right)$. 
The shape and size of CNC were characterized with transmission electron microscopy (TEM), and the specimens were prepared from its suspension in water of $0.1 \mathrm{wt} \%$. The specimen was stained by phosphotungstic acid solution $(2 \mathrm{wt} \%$ in water, $\mathrm{pH}$ was adjusted to 7.0 by $1 \mathrm{M}$ $\mathrm{NaOH})$. The TEM observation was conducted with a CM 12 Transmission Electron Microscope (Philips, Netherland).

Scanning electron microscopy (SEM) was applied to observe the microstructure of xerogels with LEO Supra-35 High-Resolution Field Emission Scanning Electron Microscope (Carl Zeiss AG, Germany) or an EVO LS15 Scanning Electron Microscope (Carl Zeiss AG, Germany).

The optical microscopy observation and birefringence measurement of specimen were performed with Eclipse E600 Polarizing Microscope from Nikon, or LV100-P Polarizing Microscope from Nikon. The retardation was measured by a Berek compensator, and calibrated by the Machine Constant Table. The birefringence was calculated by the ratio between retardation and the corresponding thickness of the specimen.

Laser Scanning Microscope (LSM) images were obtained on a VK-X100K 3D laser scanning microscope (Keyence Corporation, Germany).

The wide-angle X-ray scattering (WAXS) of the xerogels was carried out at the Shanghai Synchrotron Radiation Facility (SSRF) on the BL16B beam line with an X-ray wavelength of $0.124 \mathrm{~nm}$. The specimen was placed on a sample holder with the fiber direction perpendicular to the X-ray beam. The specimen-to-detector (PILATUS3 2M) distance was calibrated using the standard sample $\mathrm{Y}_{2} \mathrm{O}_{3}$. The beam spot size was $150(\mathrm{H}) \times 300 \mu \mathrm{m} \times \mu \mathrm{m}$. A typical acquisition time was $60 \mathrm{~s}$. All data analyses (background correction, radial and azimuthal integration) were carried out using the Xpolar software (Precision Works Inc., NY, USA). The azimuthal intensity distribution profiles along the arc that refers to the (200) reflections of the cellulose $\mathrm{I} \beta$ crystals were used to quantify the orientation of the CNC within the xerogels. ${ }^{3}$ This peak is determined to be within $2 \theta=22.0-22.4^{\circ}$. Intensity distribution profiles in the azimuthal angle $(\phi)$ were used to calculate the orientation index $(\pi)$ according to the equations: 


$$
\pi=\frac{180^{\circ}-f w h m}{180^{\circ}}
$$

where fwhm is the full width of the half-maximum of the azimuthal profiles from the selected equatorial reflection.
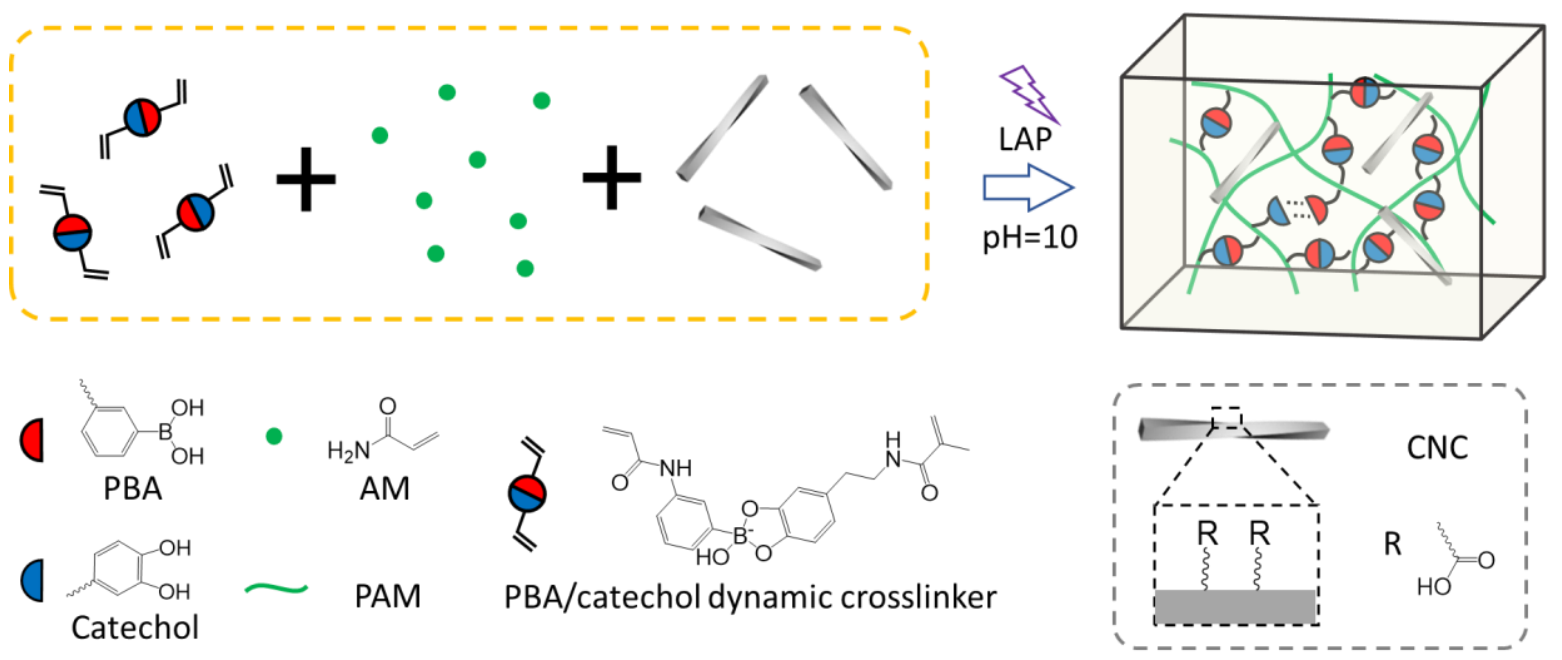

Figure S1. Dynamic hybrid hydrogels preparation with in situ polymerization. 

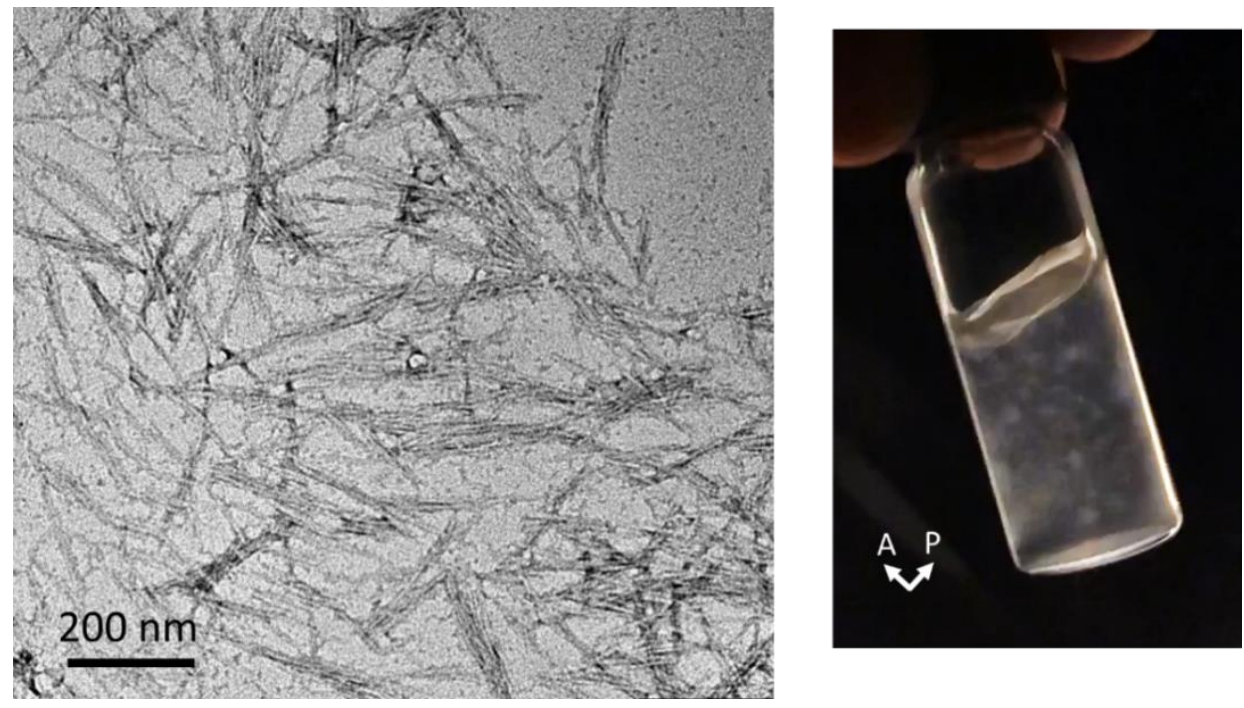

Figure S2. TEM image of $\mathrm{CNC}$ and polarized image of $\mathrm{CNC}$ suspension in DI water (concentration as $1.6 \mathrm{mg} / \mathrm{ml}$ ).

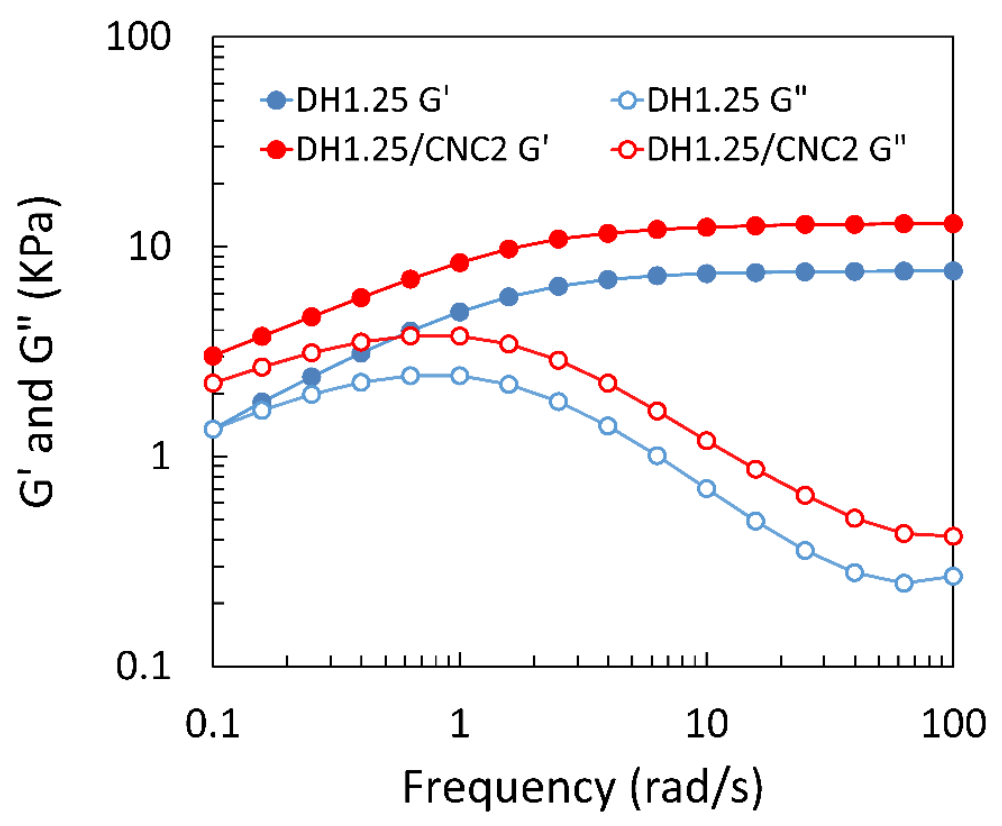

Figure S3. Oscillation shear frequency sweep of DH1.25 and DH1.25/CNC2. 

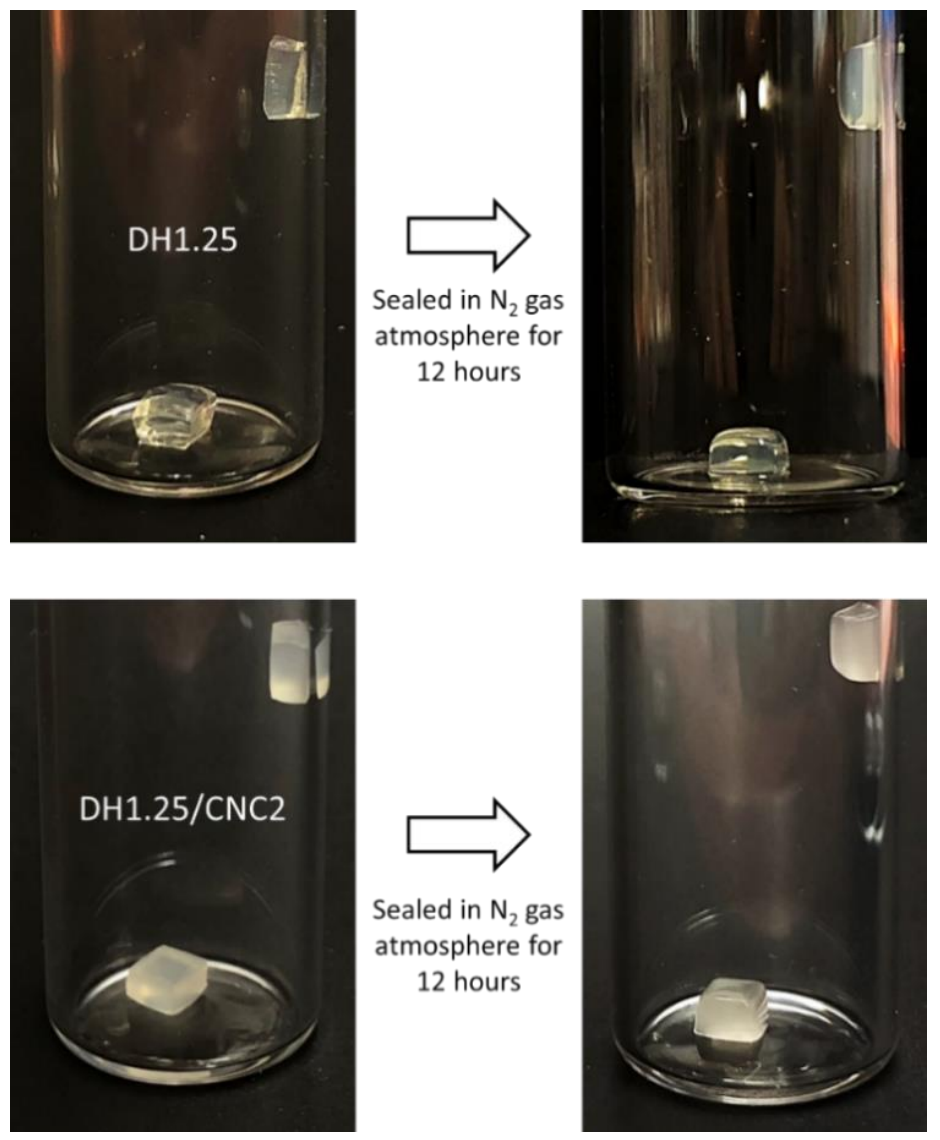

Figure S4. Shape regulation of dynamic hydrogels along the time. The edges of cubic hydrogel become round in 12 hours. 


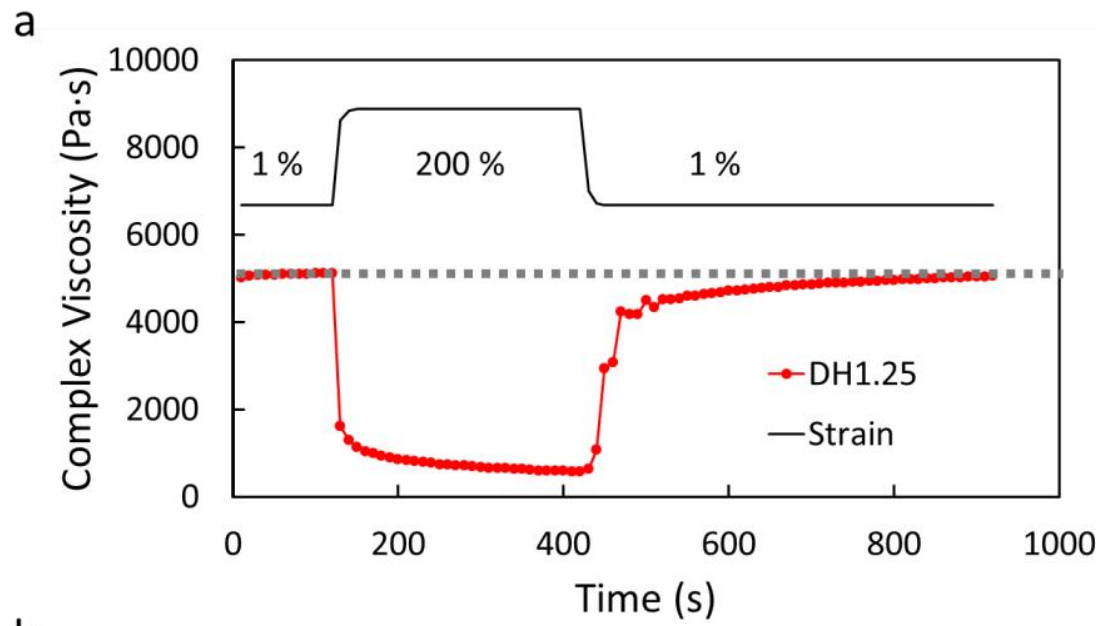

b

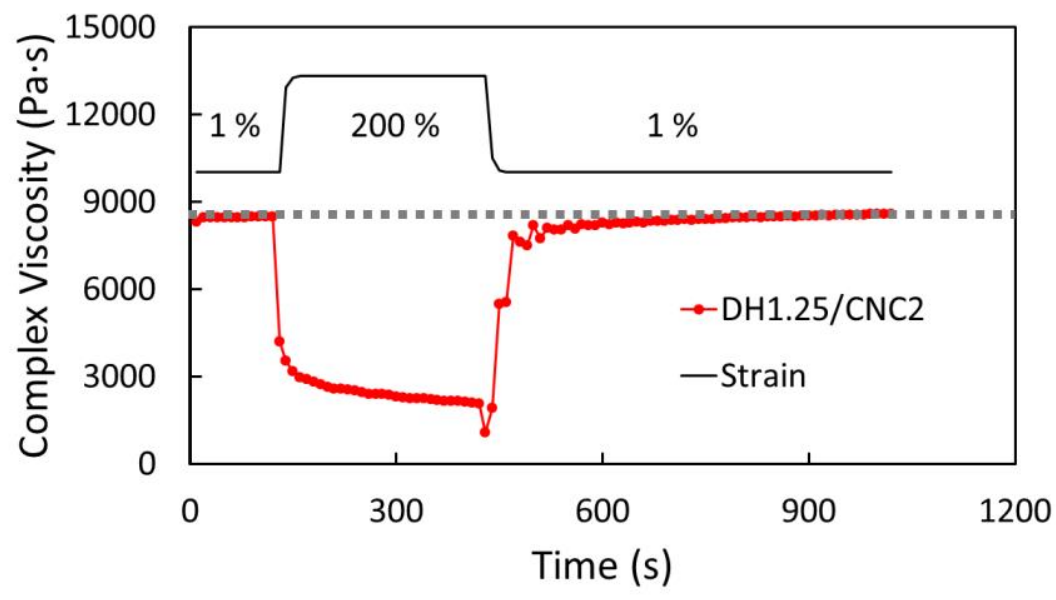

Figure S5. 3ITT shearing tests with low-high-low strain sweep of DH1.25 and DH1.25/CNC2.

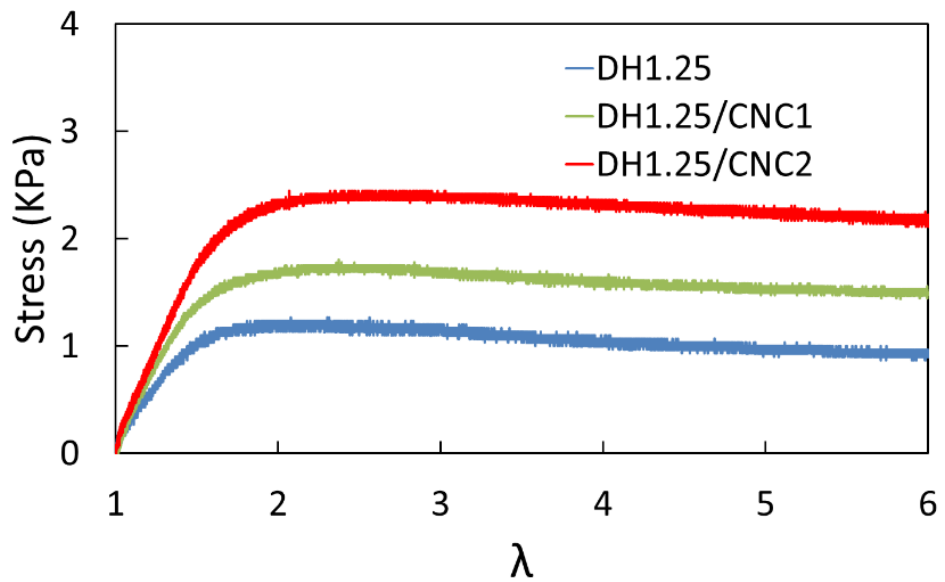

Figure S6. Tensile strain-stress curves of dynamic hydrogels with various amounts of CNC. 


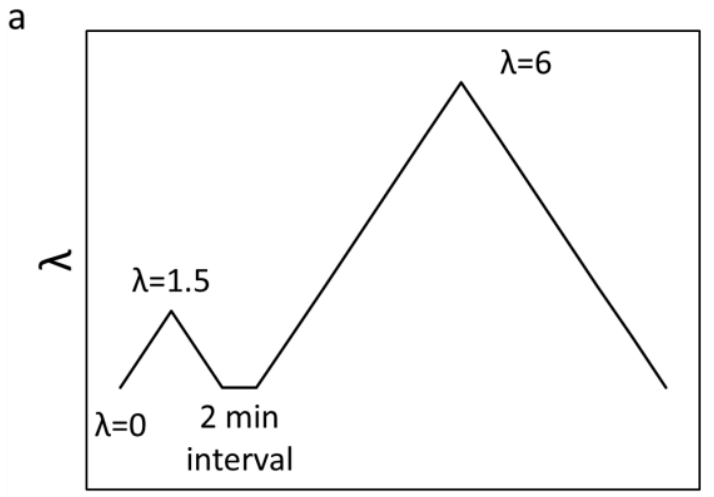

Time

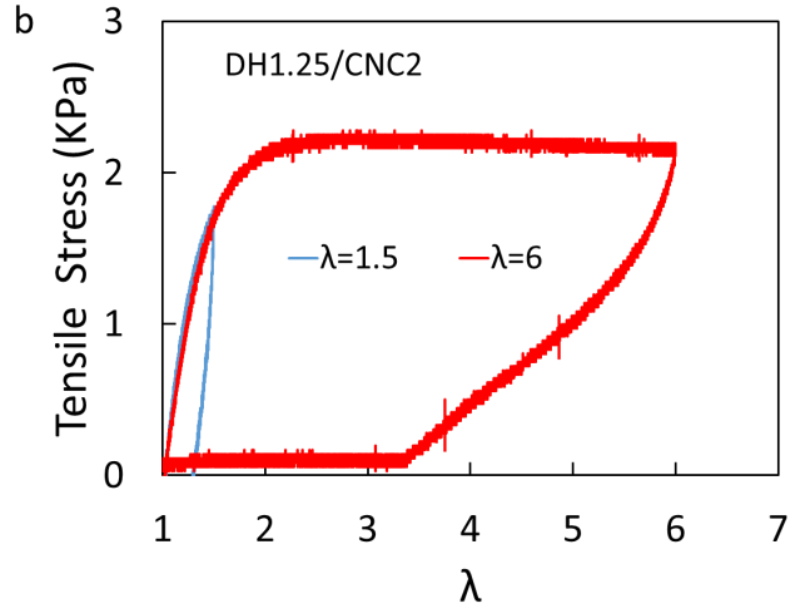

Figure S7. The hysteresis tensile tests of DH1.25/CNC2.

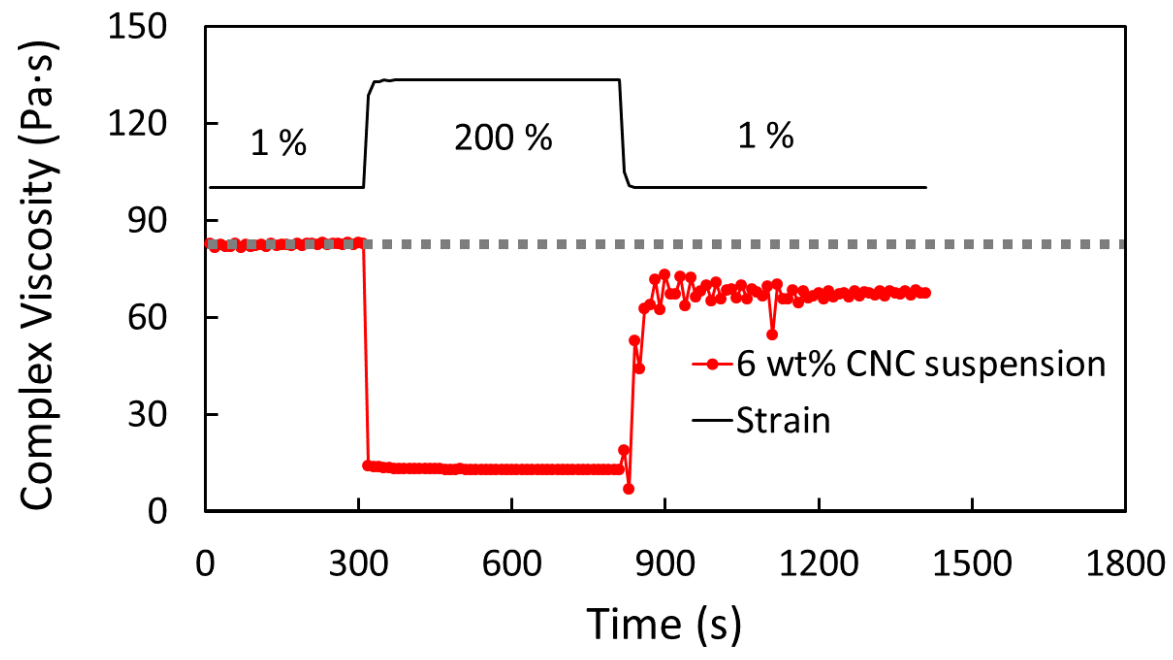

Figure S8. 3ITT shearing test with low-high-low strain sweep of $6 \mathrm{wt} \% \mathrm{CNC}$ suspension in $\mathrm{pH}=10$ borax- $\mathrm{NaOH}$ buffer. 


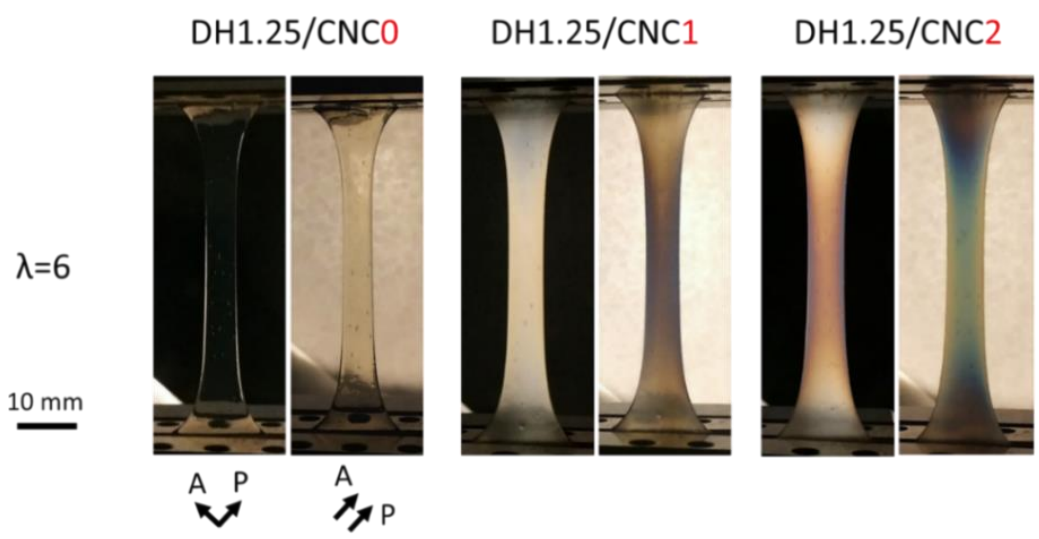

Figure S9. Stretched dynamic hydrogels with diverse amounts of CNC. The dynamic hydrogels networks show limited anisotropy, while CNC are necessary to form the interference colors in hydrogels.

a Double axials stretching of $\mathrm{DH} 1.25 / \mathrm{CNC2}$

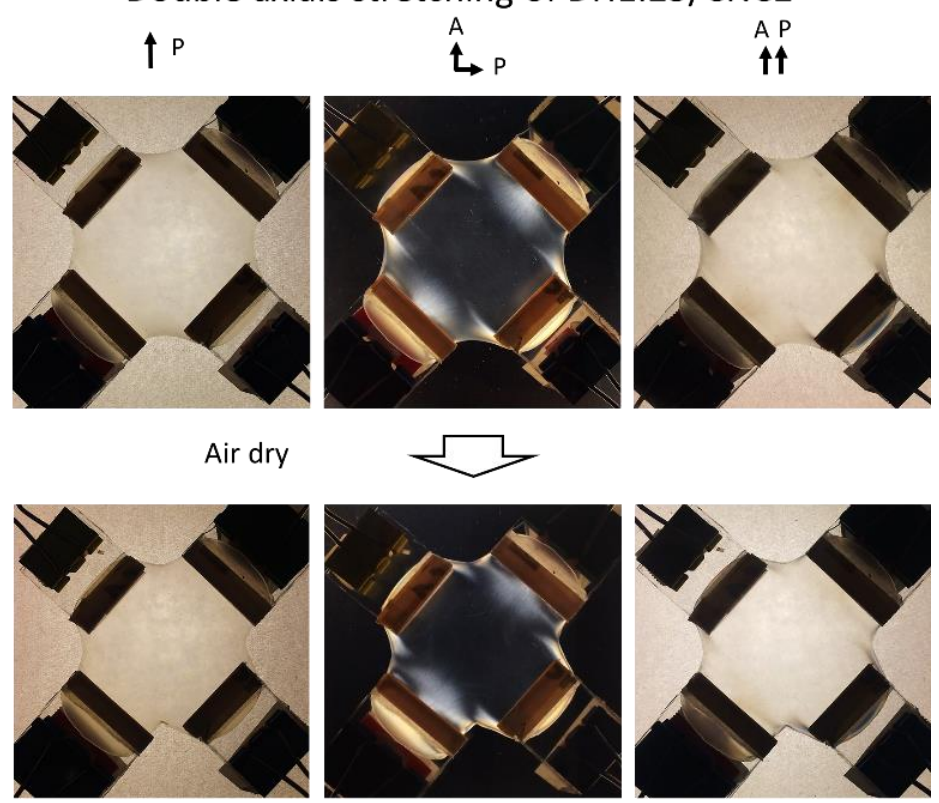

b

DH1.25/CNC2 to XG1.25/CNC2 without stretching

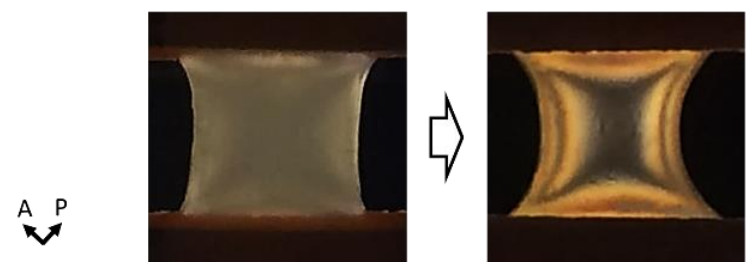

Figure S10. a) Biaxial stretching of DH1.25/CNC2. b) XG1.25/CNC2 without stretching 

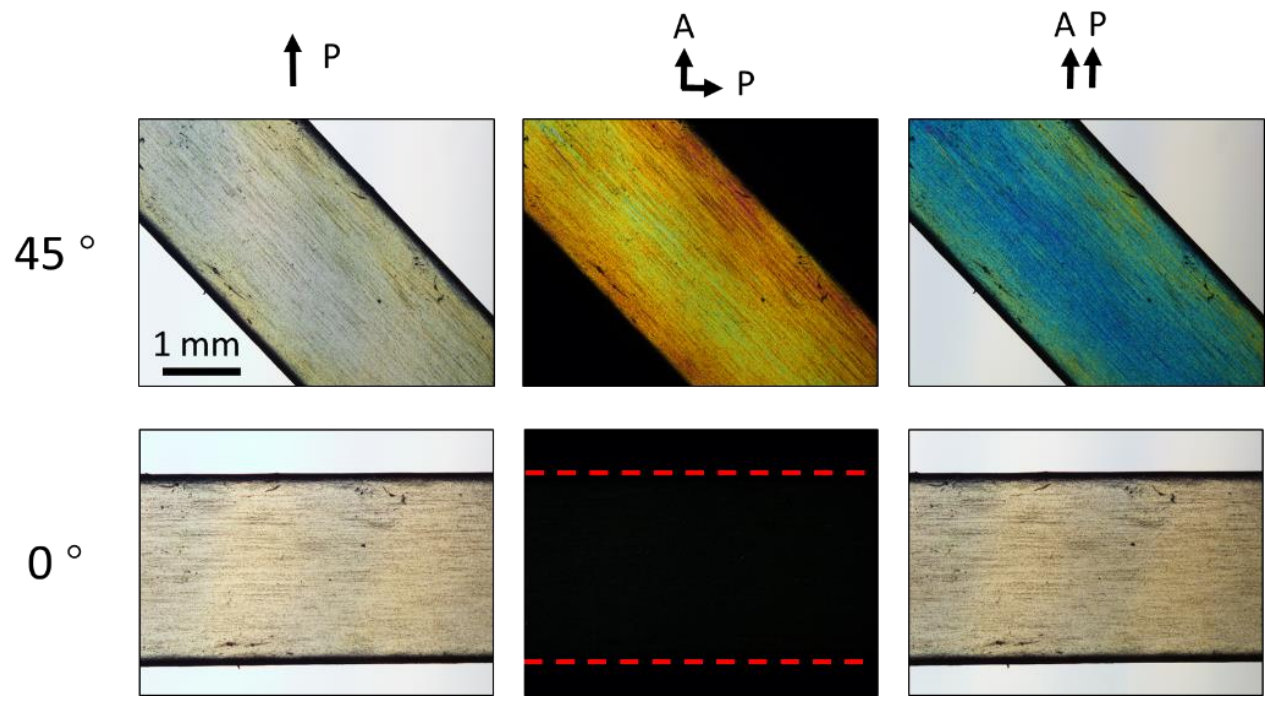

Figure S11. Polarizing optical microscopy images of XG1.25/CNC2.

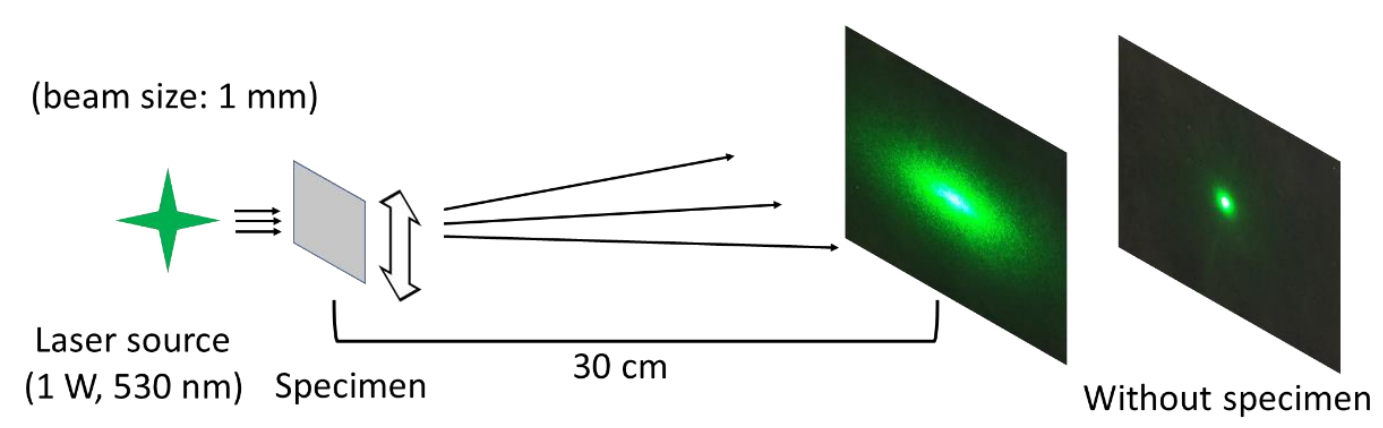

Figure S12. Schematic explanation of laser scattering equipment. 
XG1.25/CNC2 (P4)

a
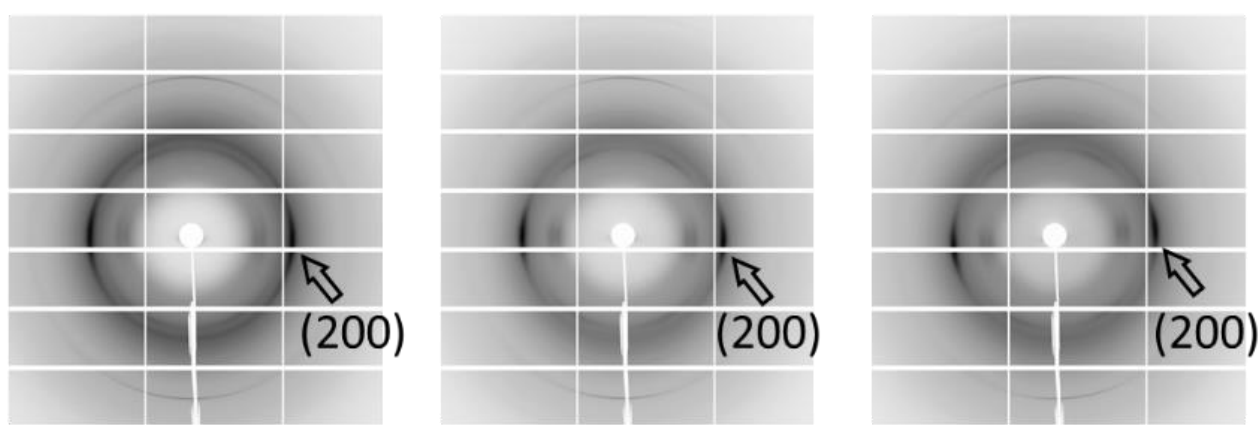

$\lambda=3$

$\lambda=6$

$\lambda=9$

b

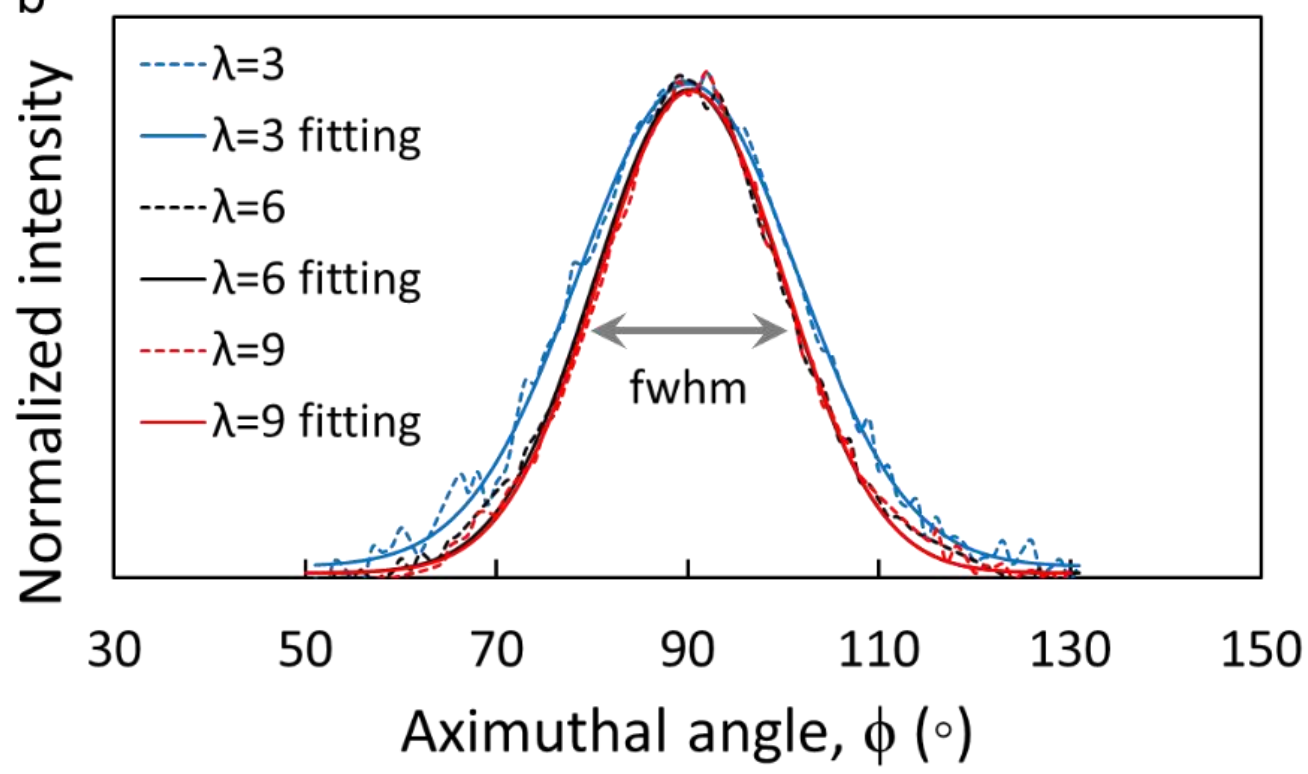

Figure S13. 2D WAXS measurements for the characterization of the CNC alignment. (a) 2D WAXS images of XG1.25/CNC2 ( $\mathrm{P} 4$, with $\lambda=3,6,9)$. (200) reflections of the cellulose $\mathrm{I} \beta$ crystals were pointed out; (b) Azimuthal intensity profiles base on (200) reflections showing narrowing fwhm with increasing elongation ratio $\lambda$. 
a Crosslinks density (mol\%) b

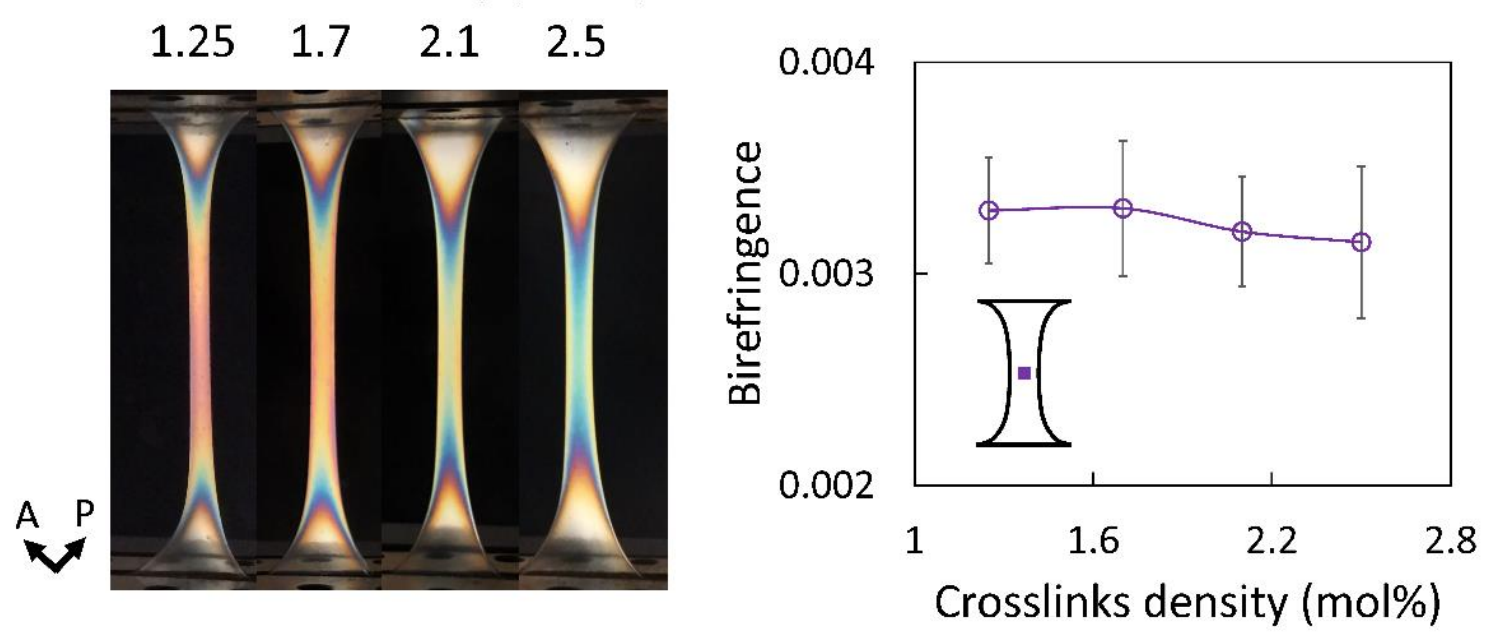

Figure S14. Xerogels prepared with various crosslinks density in dynamic hydrogels. The crosslinking density of hydrogels can be tuned with the concentration of PBA/catechol complexes. The densely crosslinked dynamic hydrogels showed less contraction in width, which was in return compensated by larger contraction in depth. Therefore, even shifted interference colors were observed, the dynamic hydrogels with various crosslinking density had similar maximum birefringence in the resulted xerogels.

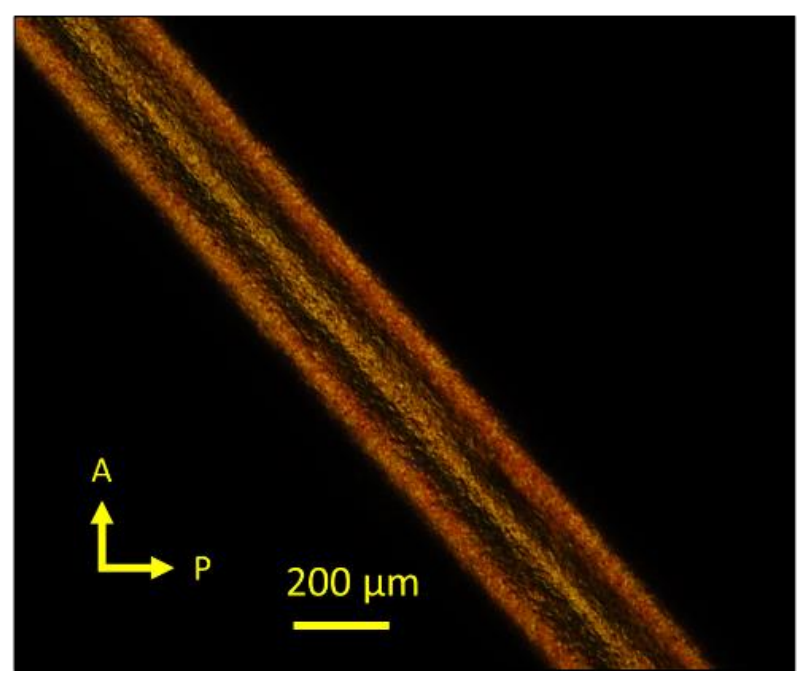

Figure S15. Polarizing optical microscope image of $\mathrm{XG1.25/CNC2} \mathrm{W}_{0} / \mathrm{D}_{0}=1$. 

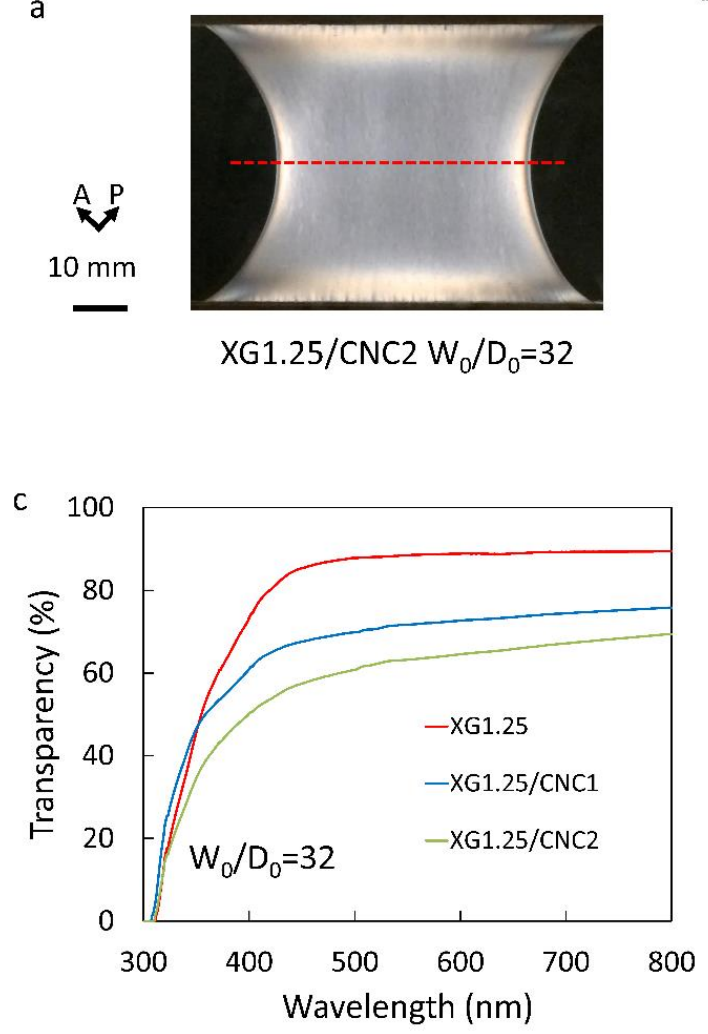

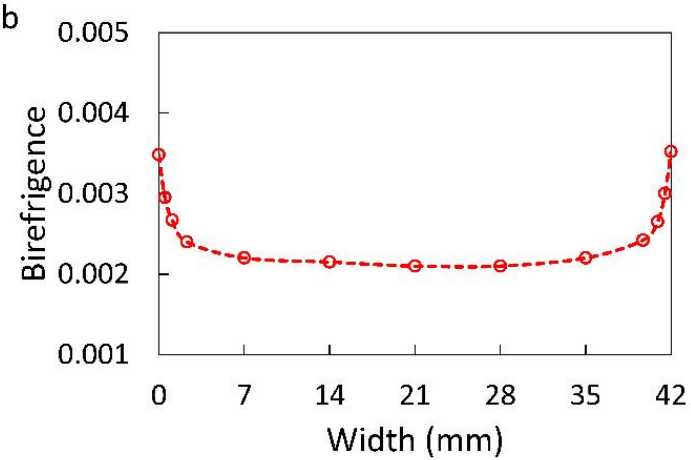

d

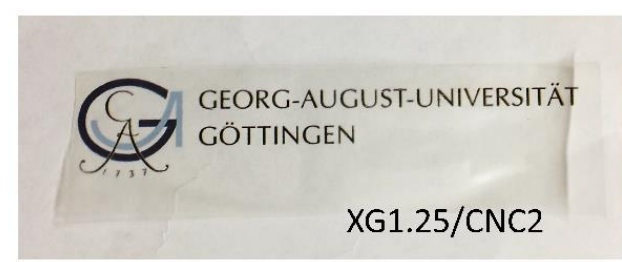

e

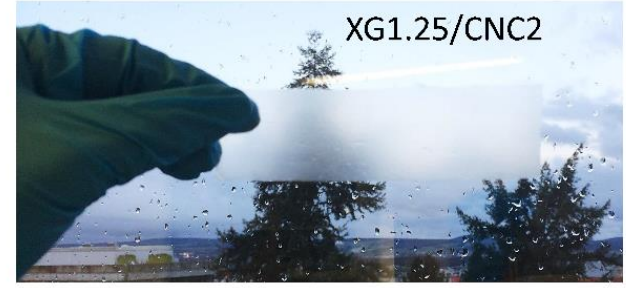

Figure S16. a) Polarizing optical microscope images of $\mathrm{XG} 1.25 / \mathrm{CNC} 2 \mathrm{~W}_{0} / \mathrm{D}_{0}=32$; $\mathrm{b}$ ) the changing of birefringence along the red dash line in a); c, d, e) Semi-transparent XG1.25/CNC2 with haze properties.
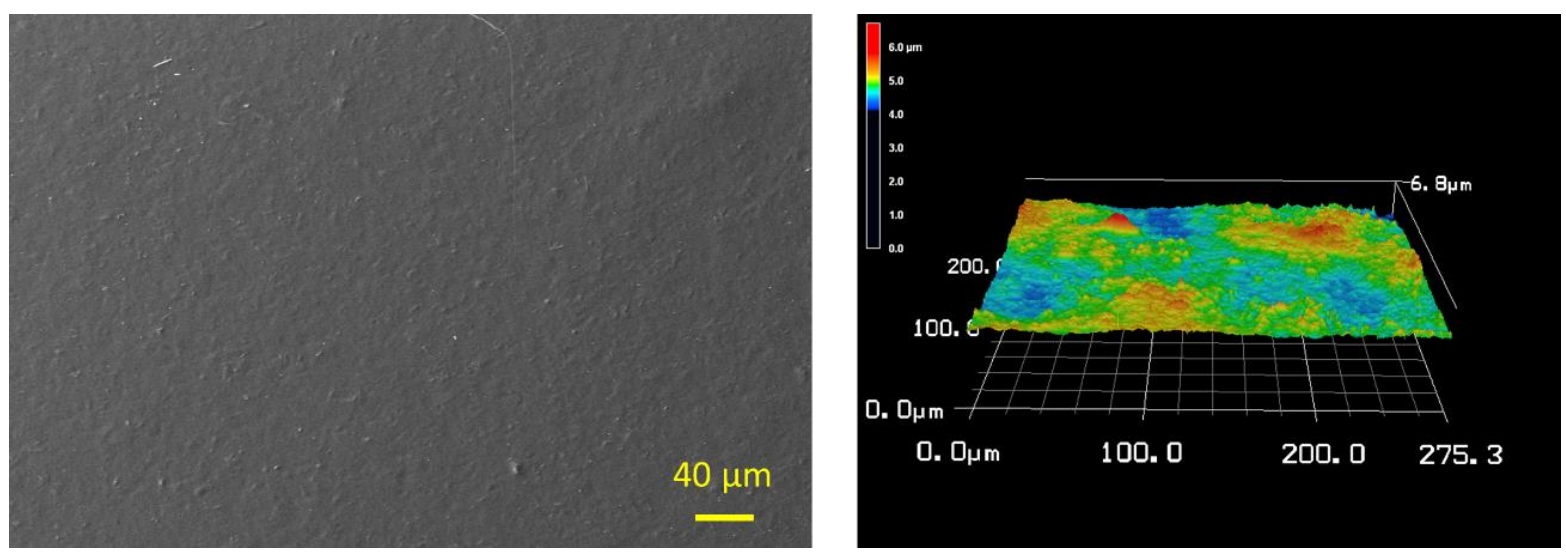

Figure S17. The surface of central area of XG1.25/CNC2 $\mathrm{W}_{0} / \mathrm{D}_{0}=32$; left: SEM top view of the films; right: surface topography observation from LSM. 

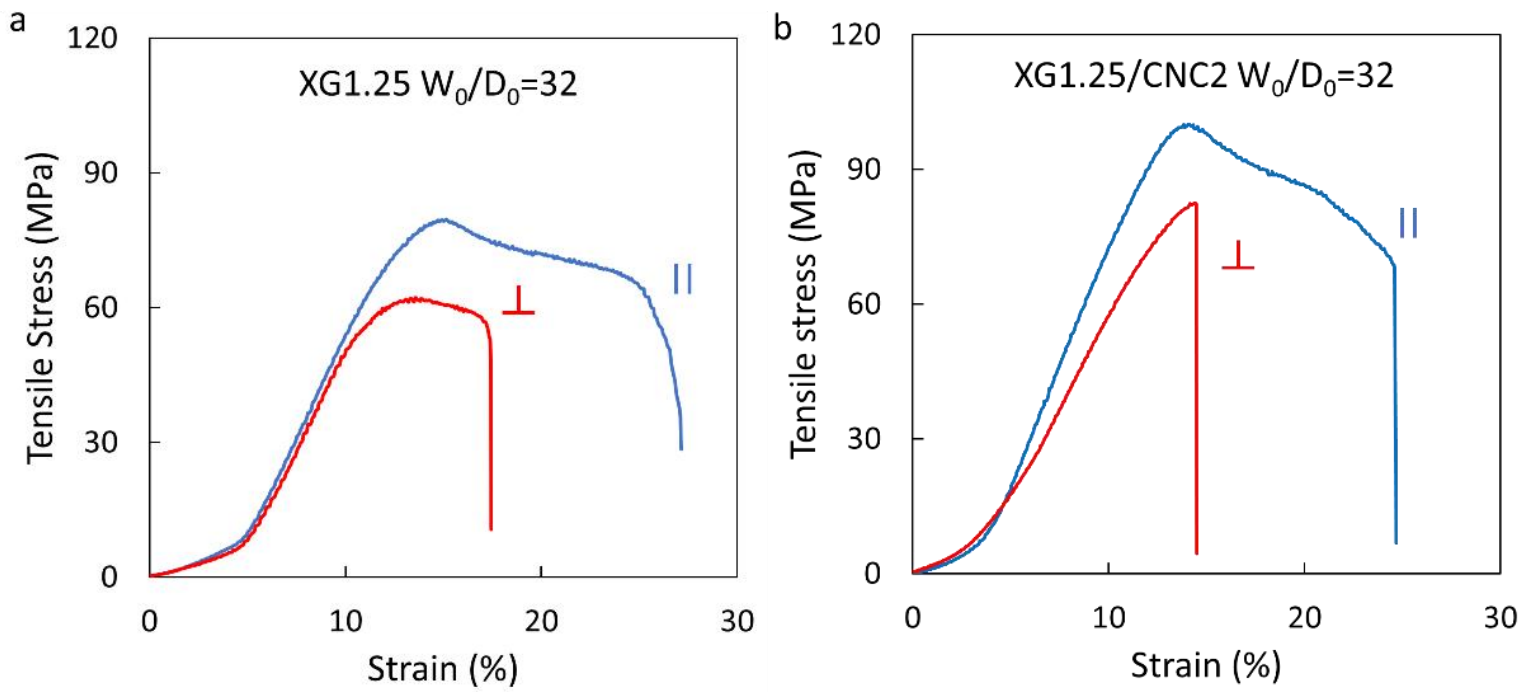

$$
X G 1.25 / C N C 2 W_{0} / D_{0}=32
$$

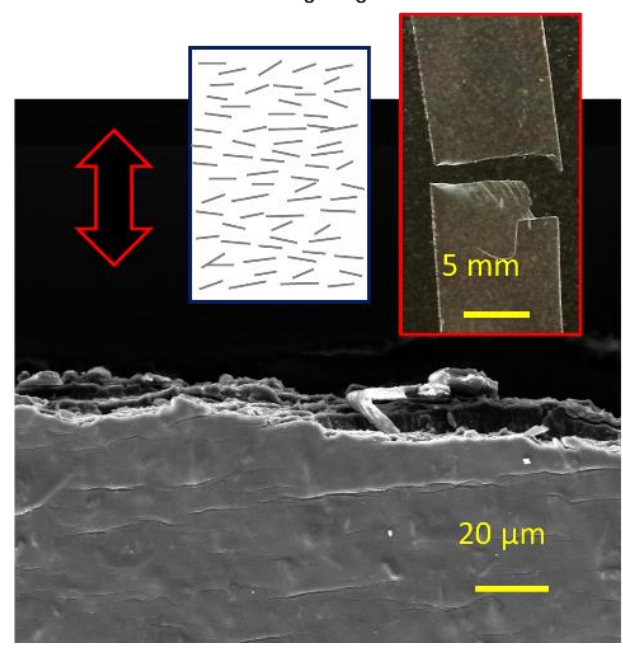

d $\quad X G 1.25 / C N C 2 W_{0} / D_{0}=32$

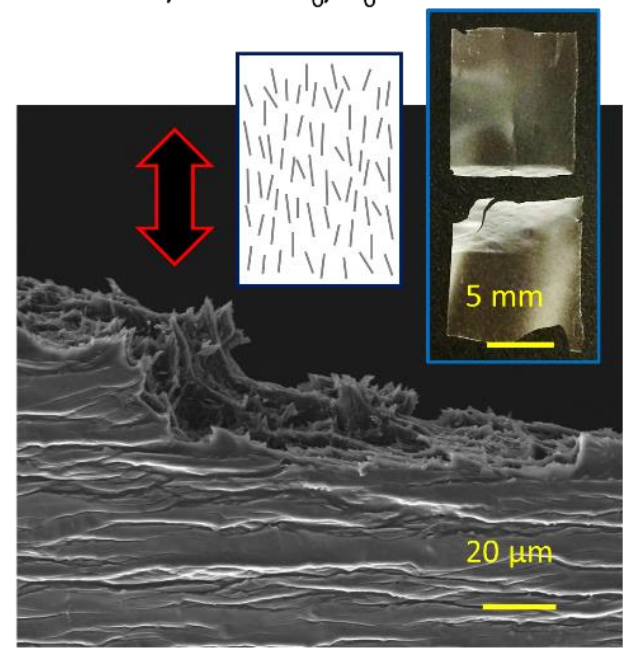

Figure S18. Anisotropic mechanical properties and fracture morphologies of XG1.25 $\mathrm{W}_{0} / \mathrm{D}_{0}=32$ and $\mathrm{XG} 1.25 / \mathrm{CNC} 2 \mathrm{~W}_{0} / \mathrm{D}_{0}=32$. 

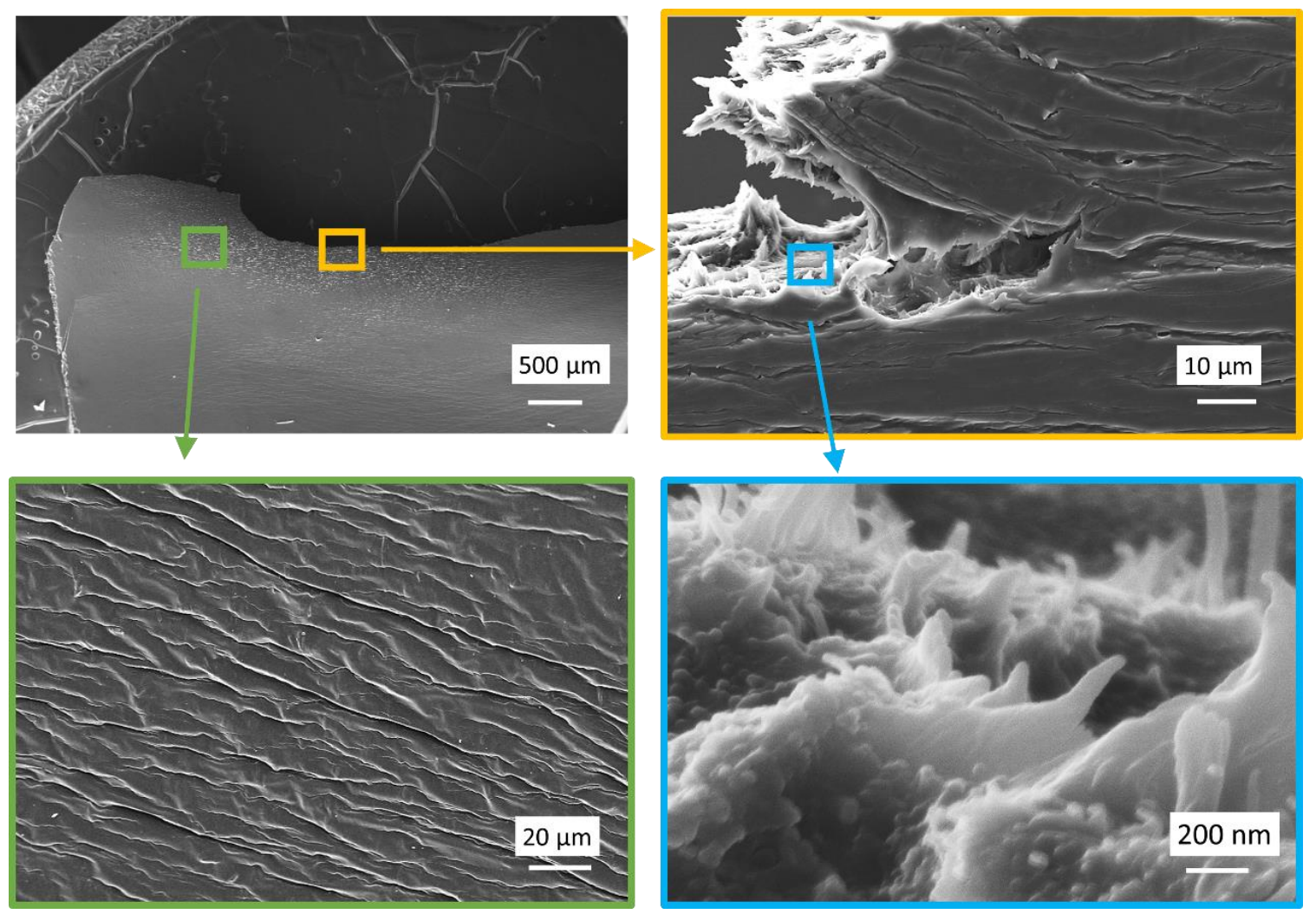

Figure S19. Fracture interface of $\mathrm{XG} 1.25 / \mathrm{CNC} 2 \mathrm{~W}_{0} / \mathrm{D}_{0}=32$ in the direction parallel to the $\mathrm{CNC}$ alignment. The wrinkled structures were formed near the fracture interface. In the zoom-in images of SEM, aligned CNC on the fracture surface can be observed. The pull-out process of $\mathrm{CNC}$ can effectively dissipate energy during stretching and promoted the ductile fracture. 


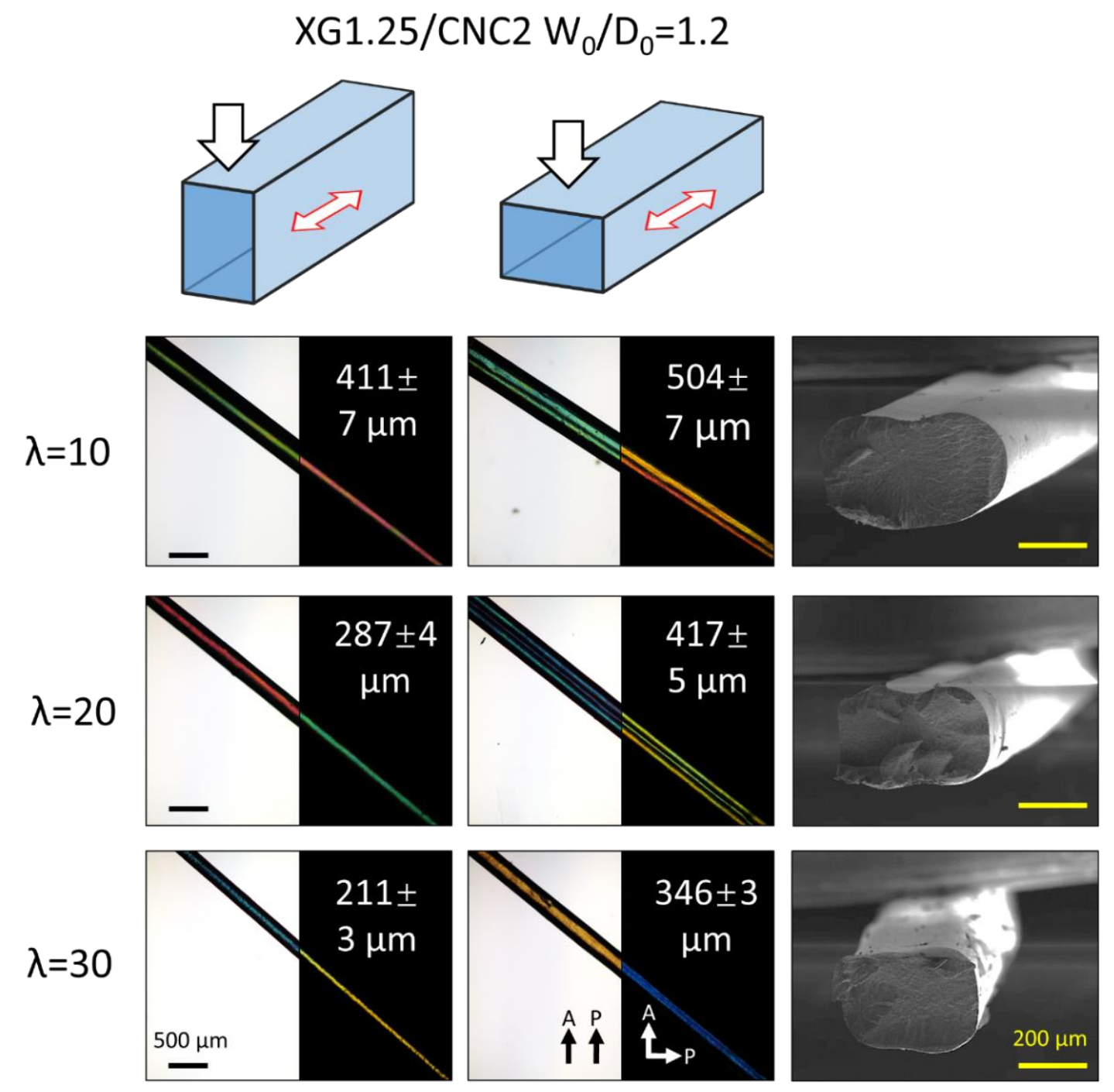

Figure S20. Fiber shaped $\mathrm{XG1.25/CNC2} \mathrm{W}_{0} / \mathrm{D}_{0}=1.2$ with different elongation ratio. 


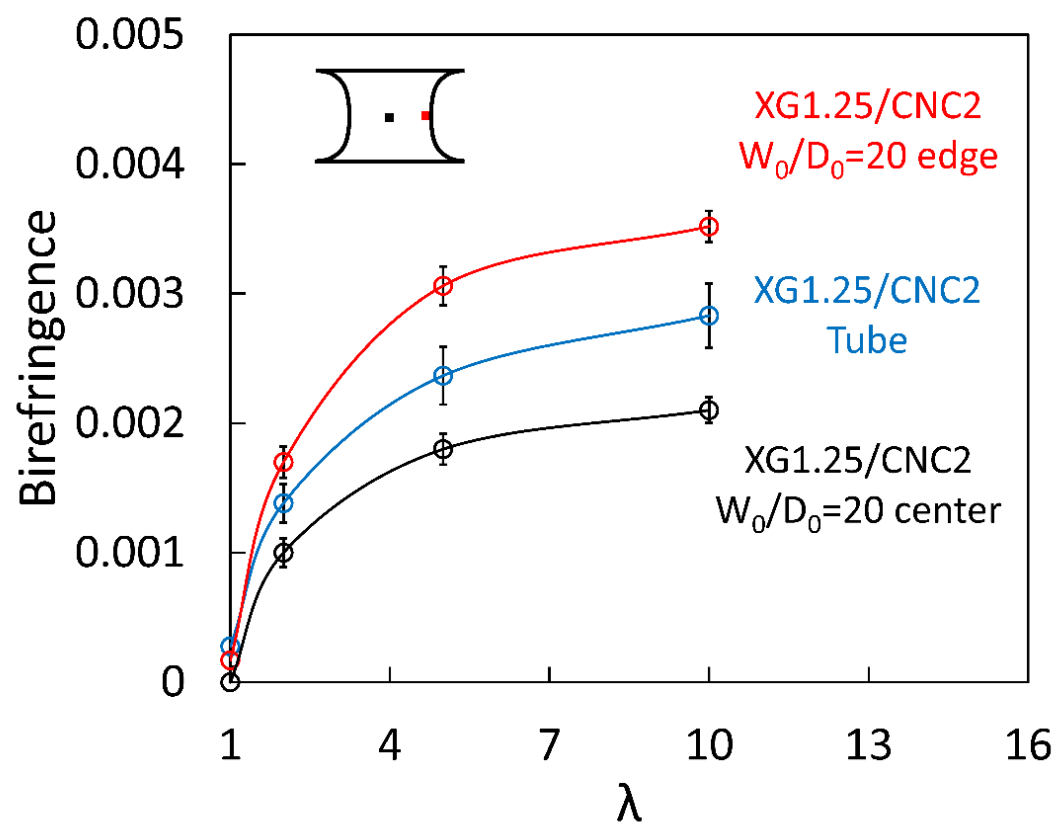

Figure S21. Birefringence comparison of XG1.25/CNC2 tubes and corresponding $\mathrm{XG} 1.25 / \mathrm{CNC} 2 \mathrm{~W}_{0} / \mathrm{D}_{0}=20$ as films.

\section{References}

(1) Lee, H.; Lee, B.P.; Messersmith, P.B. A Reversible Wet/Dry Adhesive Inspired by Mussels and Geckos. Nature 2007, 448 (7151), 338-341.

(2) Fairbanks, B.D.; Schwartz, M.P.; Bowman, C.N.; Anseth, K.S. Photoinitiated Polymerization of PEG-diacrylate with Lithium Phenyl-2,4,6-trimethylbenzoylphosphinate: Polymerization Rate and Cytocompatibility. Biomaterials 2009, 30 (35), 6702-6707.

(3) Ureña-Benavides, E. E.; Kitchens, C. L. Wide-Angle X-Ray Diffraction of Cellulose Nanocrystal-Alginate Nanocomposite Fibers. Macromolecules 2011, 44, 3478-3484. 


\section{Curriculum vitae}

\section{PERSONAL DETAILS}

Surname, Name: Huang, Heqin

Gender: Male

Nationality: Chinese

Date of Birth: 14.07.1990

Email: hhuang1@uni-goettingen.de; heqin.huang@hotmail.com

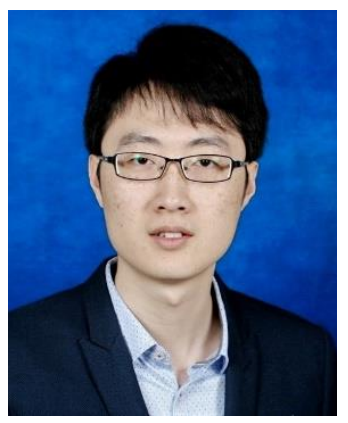

\section{EDUCATIONAL BACKGROUND}

Aug. 2015 - Mar. 2019

Georg-August-Universität Göttingen (Germany), Material Science Wood, graduated in March 2019 with a Doctoral Degree (Dr.rer.nat.).

\section{Sep. 2013 - Jul.2014}

City University of Hong Kong (Hong Kong, China), Materials Engineering \& Nanotechnology, graduated in July 2014 with a Master Degree.

Sep. 2009 - Jun. 2013

University of Jinan (China), Polymer Materials \& Engineering, graduated in June 2013 with a Bachelor Degree.

\section{PUBLICATIONS}

1. Heqin Huang, Xiaojie Wang, Jinchao Yu, Ye Chen, Hong Ji, Yumei Zhang, Florian Rehfeldt, Yong Wang, and Kai Zhang. Liquid-behaviors-assisted fabrication of multidimensional birefringent materials from dynamic hybrid hydrogels. ACS Nano, 2019, DOI: $10.1021 / \mathrm{acsnano.9b00551.}$

2. Xiaojie Wang, Heqin Huang, Huan Liu, Florian Rehfeldt, Xiaohui Wang, and Kai Zhang. Multi-responsive bilayer hydrogel actuators with programmable and precisely tunable 
motions. Macromolecular Chemistry and Physics, 2019, 220, 1800562.

3. Heqin Huang, Xiaojie Wang, Florian Rehfeldt, and Kai Zhang. Dually heterogeneous hydrogels via dynamic and supramolecular cross-links tuning discontinuous spatial ruptures. ACS Sustainable Chemistry \& Engineering, 2018, 6 (3), 4294-4301.

4. Heqin Huang, Yonggui Wang, Xiaojie Wang, Florian Rehfeldt, and Kai Zhang. Robust heterogeneous hydrogels with dynamic nanocrystal-polymer interface. Macromolecular Rapid Communications, 2017, 38, 1600810.

5. Qian Feng, Sien Lin, Kunyu Zhang, Chaoqun Dong, Tianyi Wu, Heqin Huang, Xiaohui Yan, Li Zhang, Gang Li, and Liming Bian. Sulfated hyaluronic acid hydrogels with retarded degradation and enhanced growth factor retention promote hMSC chondrogenesis and articular cartilage integrity with reduced hypertrophy. Acta Biomaterialia, 2017, 53, 329342.

6. Heqin Huang, Jianbin $\mathrm{Xu}$, Kongchang Wei, Yang $\mathrm{J} \mathrm{Xu}$, Chun Kit K Choi, Meiling Zhu, and Liming Bian. Bioactive nanocomposite poly (ethylene glycol) hydrogels crosslinked by multifunctional layered double hydroxides nanocrosslinkers. Macromolecular Bioscience, 2016, 16 (7), 1019-1026.

7. Jianbin $\mathrm{Xu}$, Jinming Li, Sien Lin, Tianyi Wu, Heqin Huang, Kunyu Zhang, Yuxin Sun, Kelvin WK Yeung, Gang Li, and Liming Bian. Nanocarrier - Mediated codelivery of small molecular drugs and siRNA to enhance chondrogenic differentiation and suppress hypertrophy of human mesenchymal stem cells. Advanced Functional Materials, 2016, 26 (15), 2463-2472.

8. Heqin Huang, Yuanyuan Ding, Xilu Chen, Zhiyong Chen, and Xiang Zheng Kong. Synthesis of monodisperse micron-sized poly (divinylbenzene) microspheres by solvothermal precipitation polymerization. Chemical Engineering Journal, 2016, 289, $135-141$

9. Heqin Huang, Rui Chen, Jiale Ma, Li Yan, Yingqi Zhao, Yu Wang, Wenjun Zhang, Jun Fan, and Xianfeng Chen. Graphitic carbon nitride solid nanofilms for selective and recyclable sensing of $\mathrm{Cu}^{2+}$ and $\mathrm{Ag}^{+}$in water and serum. Chemical Communications, 2014, $50(97), 15415-15418$. 


\section{CONFERENCES}

1. Heqin Huang and Kai Zhang. Dynamic heterogenous hydrogels constructed by cellulose nanocrystals. Cellulose Symposium of ZELLCHEMING-Expo. Jun. 27 $7^{\text {th }}-28^{\text {th }} 2018$. Frankfurt, Germany. (Oral presentation)

2. Heqin Huang, Xiaojie Wang, Florian Rehfeldt and Kai Zhang. Dually heterogeneous hydrogels via dynamic and supramolecular crosslinks tuning discontinuous spatial ruptures. Bordeaux Polymer Conference. May $28^{\text {th }}-31^{\text {st }} 2018$, Bordeaux, France. (Oral presentation)

3. Heqin Huang and Kai Zhang. Dynamic heterogeneous hydrogels via supramolecular approaches. Biennial Meeting of the GDCh-Division of macromolecular Chemistry. Sept. $11^{\text {th }}-13^{\text {th }} 2016$. Halle (Saale), Germany. (Poster presentation) 